AFRL-IF-RS-TR-2005-54

Final Technical Report

February 2005

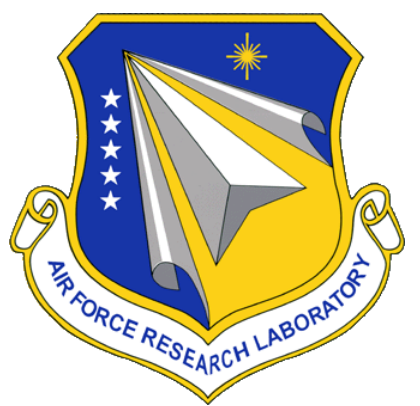

\title{
AN ADVANCED PLATFORM FOR BIOMOLECULAR DETECTION AND ANALYSIS SYSTEMS
}

University of Wisconsin

Sponsored by

Defense Advanced Research Projects Agency

DARPA Order No. E117

APPROVED FOR PUBLIC RELEASE; DISTRIBUTION UNLIMITED.

The views and conclusions contained in this document are those of the authors and should not be interpreted as necessarily representing the official policies, either expressed or implied, of the Defense Advanced Research Projects Agency or the U.S. Government.

\author{
AIR FORCE RESEARCH LABORATORY \\ INFORMATION DIRECTORATE \\ ROME RESEARCH SITE \\ ROME, NEW YORK
}




\section{STINFO FINAL REPORT}

This report has been reviewed by the Air Force Research Laboratory, Information Directorate, Public Affairs Office (IFOIPA) and is releasable to the National Technical Information Service (NTIS). At NTIS it will be releasable to the general public, including foreign nations.

AFRL-IF-RS-TR-2005-54 has been reviewed and is approved for publication

APPROVED:

/S/

THOMAS RENZ

Project Engineer

FOR THE DIRECTOR:

/s/

JAMES A. COLLINS, Acting Chief

Advanced Computing Division

Information Directorate 


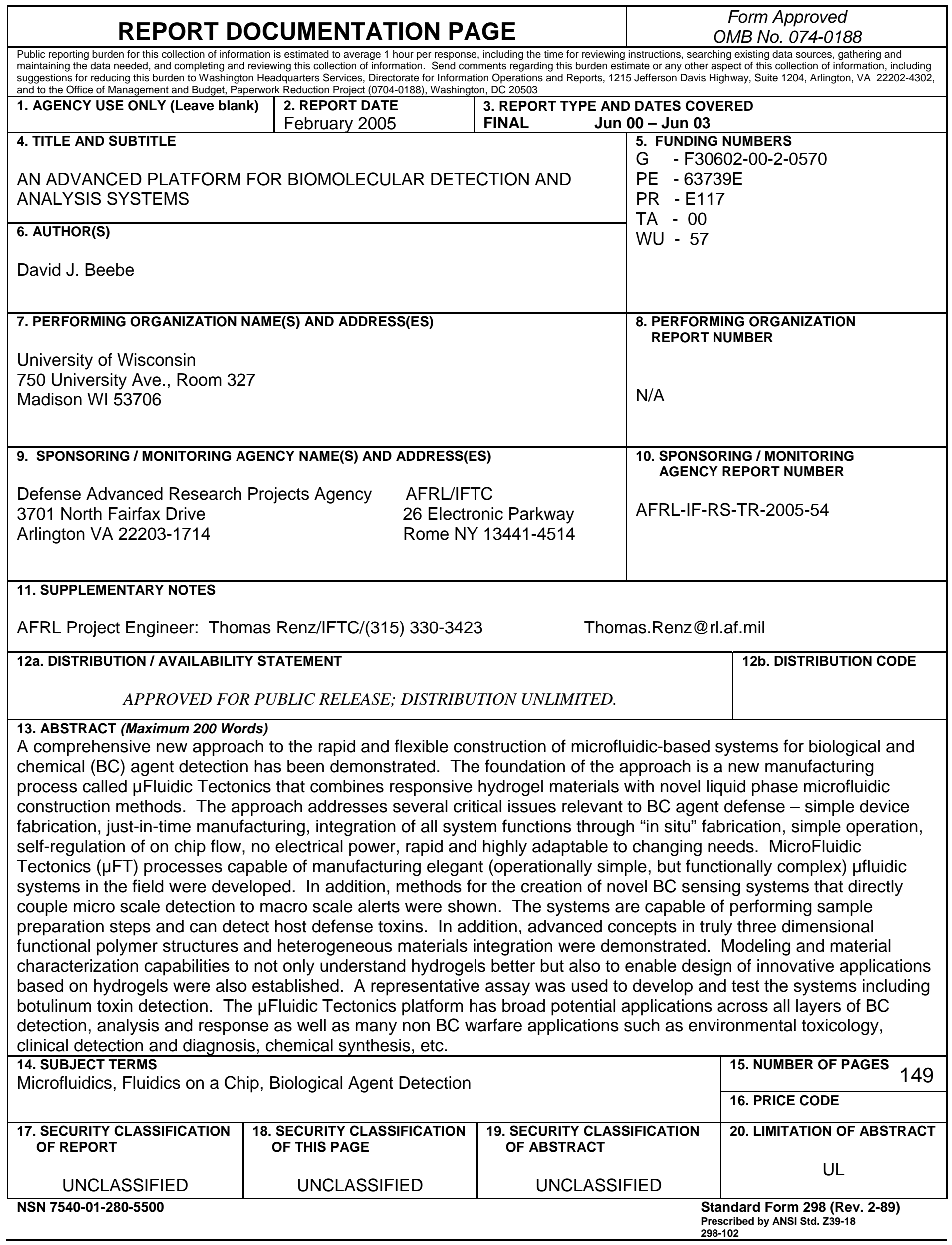




\section{Table of Contents}

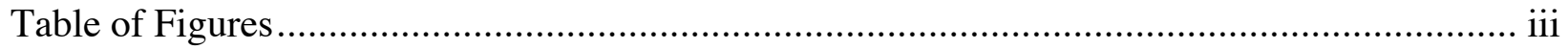

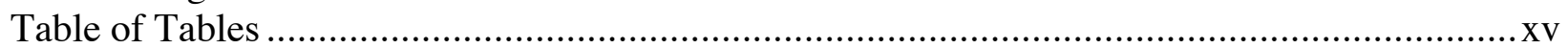

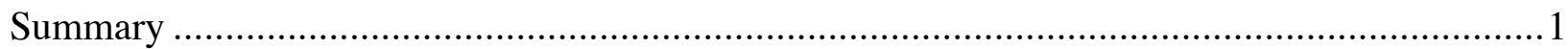

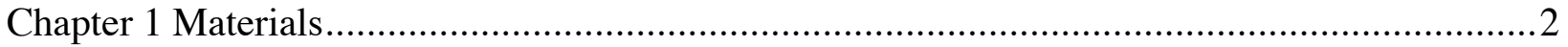

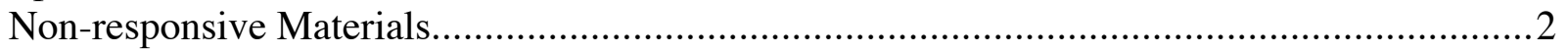

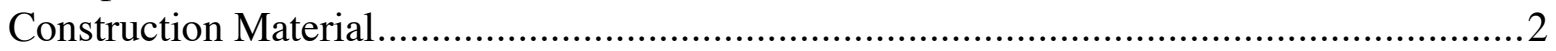

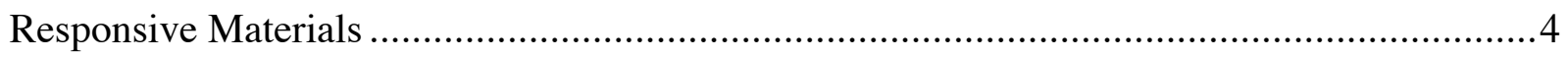

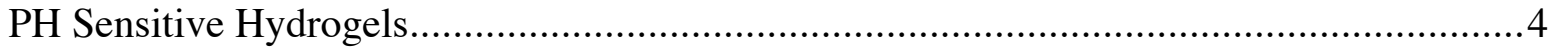

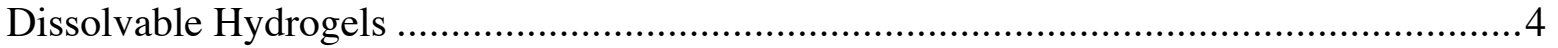

Temperature Sensitive Hydrogels ......................................................................... 4

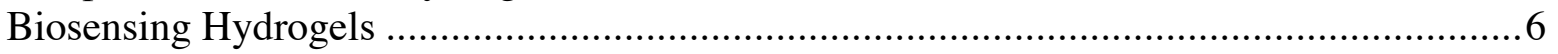

Surface Studies of Hydrogels ...................................................................... 7

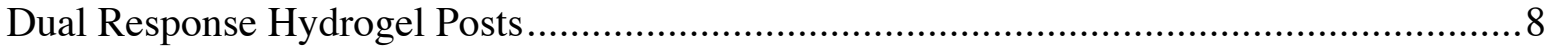

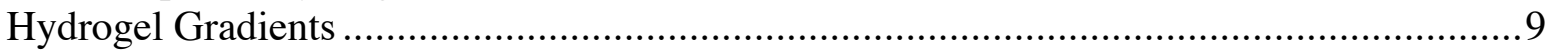

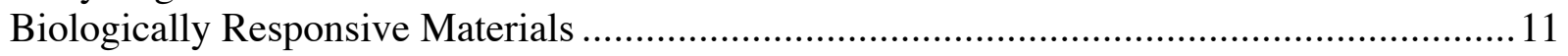

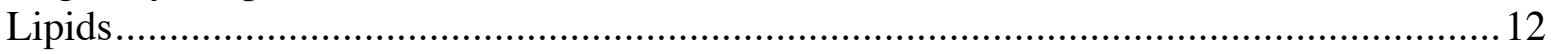

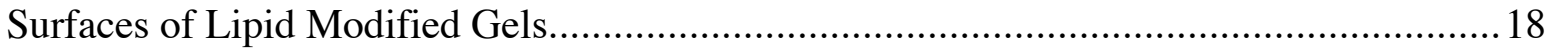

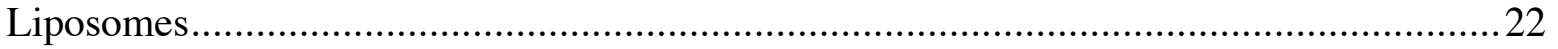

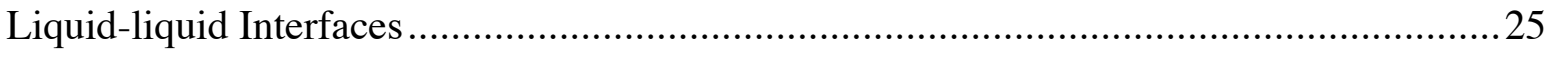

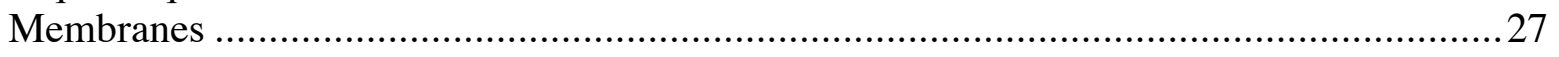

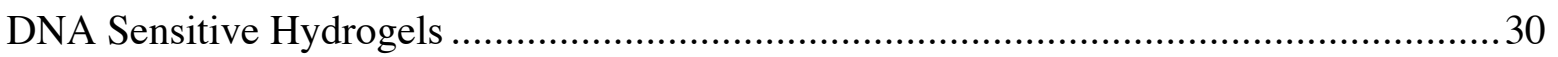

Botulinum Sensitive Hydrogels ................................................................... 31

Estrogen Receptor (ER) Responsive Hydrogels ................................................ 33

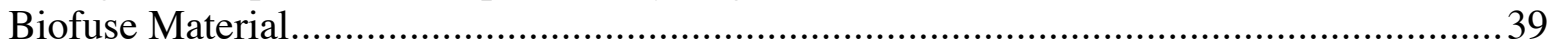

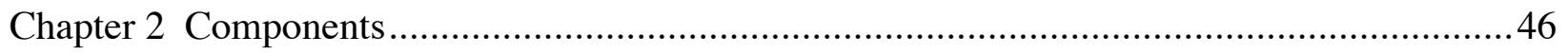

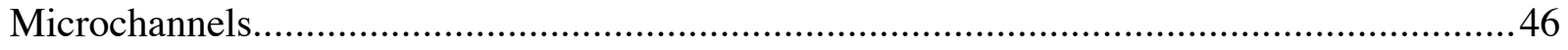

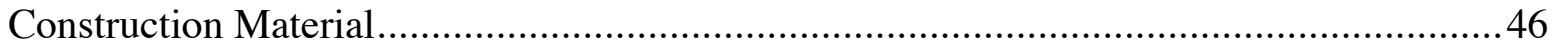

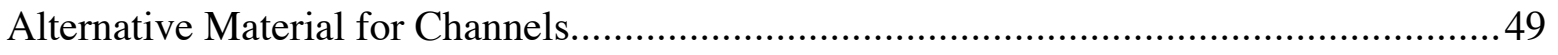

Dissolvable Hydrogels ..................................................................................... 51

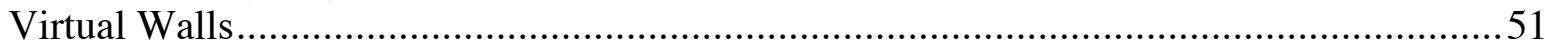

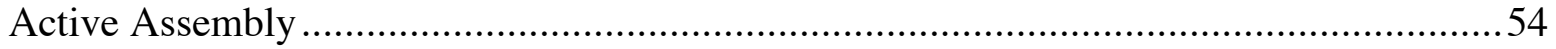

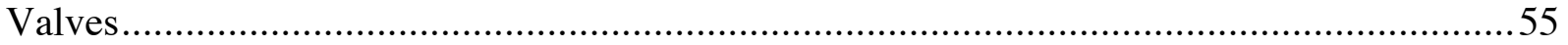

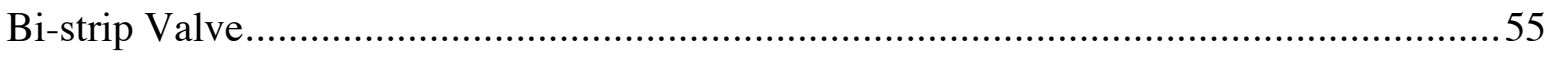

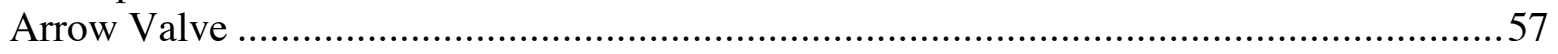

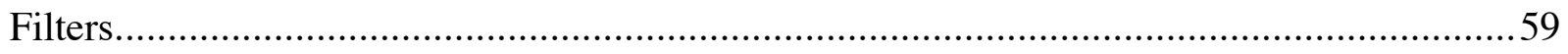

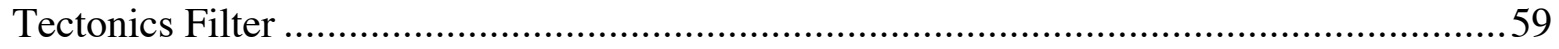

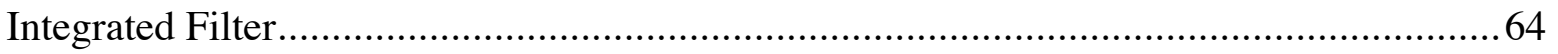

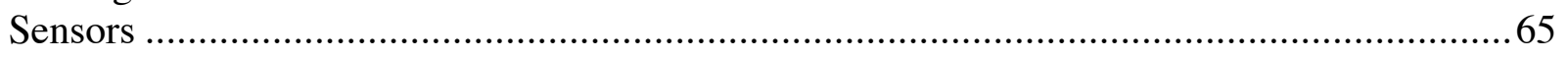

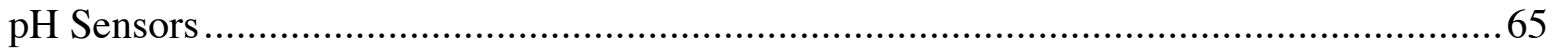

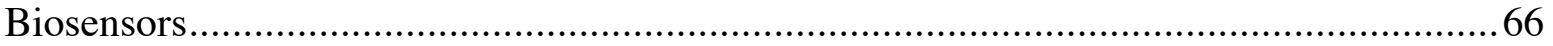




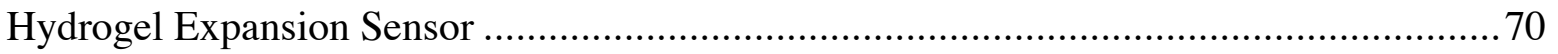

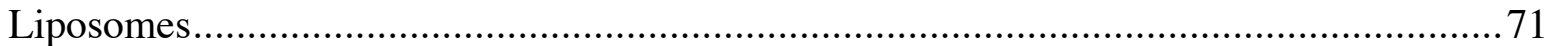

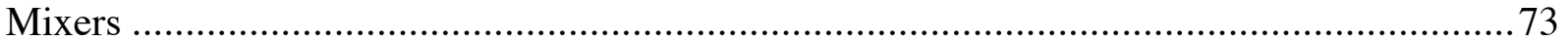

Passive Mixer.................................................................................................. 74

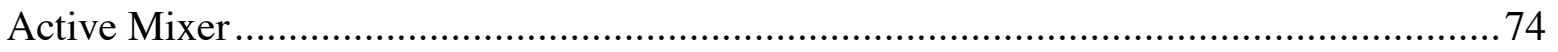

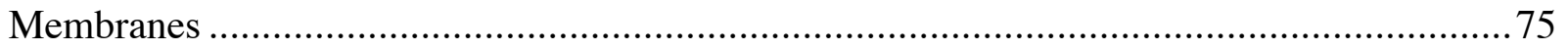

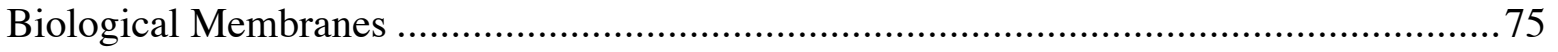

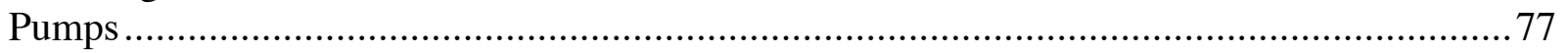

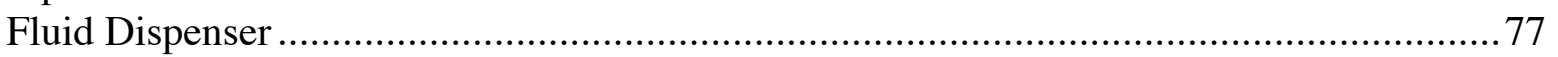

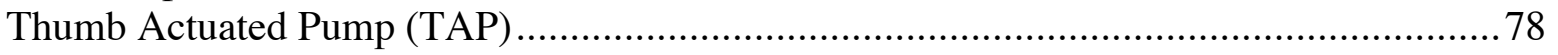

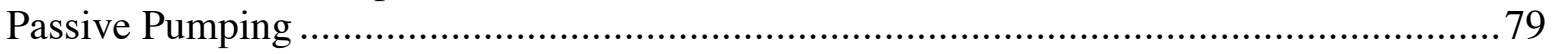

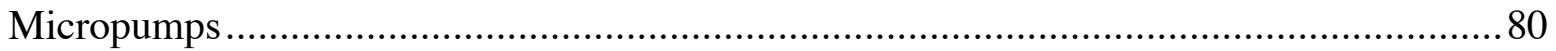

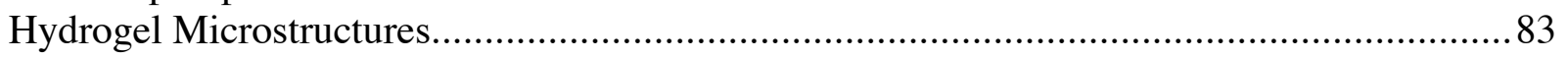

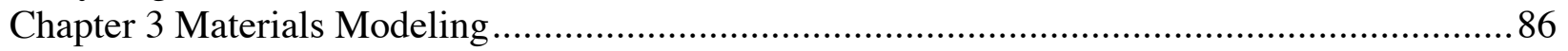

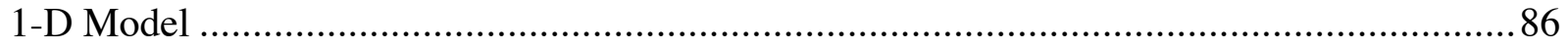

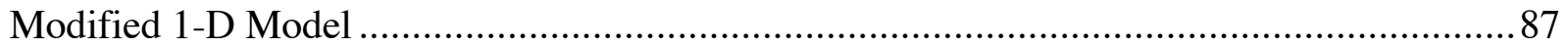

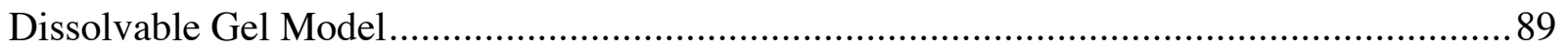

Electrically Responsive Gel Model ........................................................................... 91

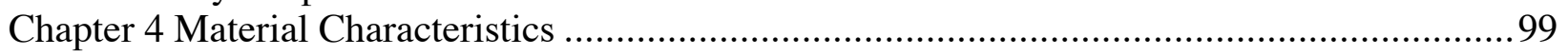

Hydrogel Properties ........................................................................................... 99

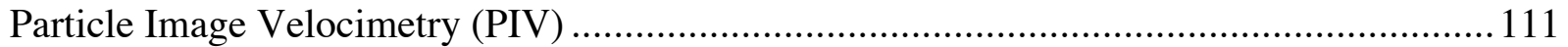

Electric Field Responsive Hydrogels ....................................................................... 113

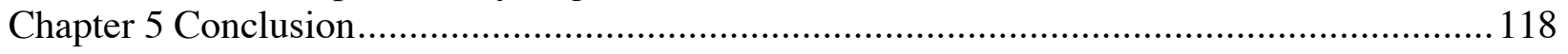

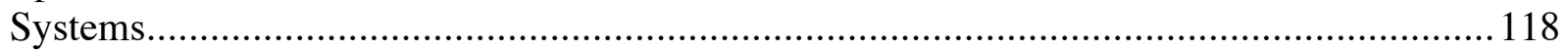

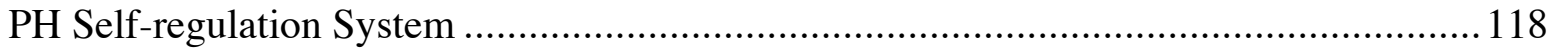

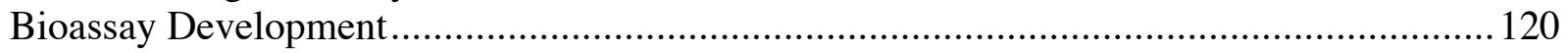

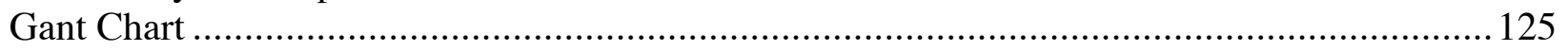

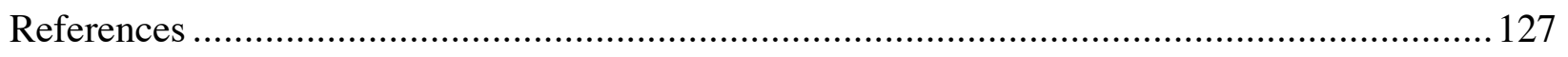

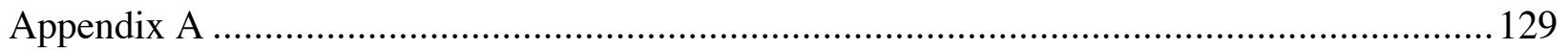




\section{Table of Figures}

Figure 1.1. Microchannels created using IBA based "construction" material. Bars $=500 \mu \mathrm{m}$....2

Figure 1.2. Structures showing resolution obtained by using construction material with features down to 25 microns and channel spacing of 100 microns...........................................2

Figure 1.3. A schematic of the surface treatment inside universal glass channels. The silane coupling agent creates covalent bonds between the glass substrate and the construction material during photopolymerizaton

Figure 1.4. Microchannels created with $\mu \mathrm{FT}$ that remained leakage-free. Bars $=500 \mu \mathrm{m}$. .......3

Figure 1.5. Two $\mathrm{pH}$ responsive hydrogel posts are shown inside a channel both before (a) and after (b) being exposed to higher $\mathrm{pH}$....

Figure 1.6. A photopolymerizable hydrogel with disulfide crosslinks dissolves in the present of DTT.

\section{.5}

Figure 1.7. Temperature sensitivity of BMAAm/DMAAm/ASME gels at different polymerization times $(10-60$ seconds $)$ for different temperatures $\left(5^{\circ} \mathrm{C}, 23^{\circ} \mathrm{C}\right.$, and $\left.40^{\circ} \mathrm{C}\right)$ Gels were photopolymerized through a $400 \mu \mathrm{m}$ diameter circle.

Figure 1.8. A hydrogel post containing antibody with HRP conjugate after exposure to 4chloro-1-napthol (4C1N) shows a blue color (a) and after exposure to 3-amino-9ethylcarbzole (AEC) shows a red color (b). Post sizes are $300 \mu \mathrm{m}$.

Figure 1.9. Color development of sandwich ELISA in hydrogel after the addition of substrate over time.

Figure 1.10. An optical micrograph of a GMM-co-AA hydrogel with a spike-circle design (a). Reductive amination of FITC-BSA to a $\mathrm{NaIO}_{4}$ oxidized hydrogel (b) and a non-oxidized hydrogel (c) was attempted using $\mathrm{NaCNBH}_{3}$. Only the oxidized hydrogel retained the fluorescent protein after washing.

Figure 1.11. Swelling of hydrogels with $80 \%$ HEMA and $20 \%$ ionizable monomers MAA and DMAEMA in different ratios [1:1 $\diamond), 2: 1(\square)$, and 1:2 $(\pi)]$. Hydrogels were bathed in buffers from $\mathrm{pH} 2$ to $\mathrm{pH} 12$.

Figure 1.12. Dual responsive hydrogel posts are shown with different core shell ratios of swellable hydrogels.

Figure 1.13. A hydrogel structure made inside a microchannel with a gradient of crosslinker is shown in the contracted (left), expanded (right) state.

Figure 1.14. A schematic view of the cross section of the channel is shown above. The two bottom flows generate the molecular gradient and the third top flow functions as the "spacer" allowing for polymerization of the bottom surface only.

Figure 1.15. Surface polymerization of an acrylamide gel (fluorescent beads) and corresponding white image with blue water indicates surface layer polymerization (left). A gradient surface polymerization of acrylamide gel (fluorescent beads in lower stream) and corresponding white image with green water are shown (right).

Figure 1.16. Cell-gel fabrication shows a microgel cylinder (a) is polymerized in a microchanel. Fatty acid chains (b) are covalently linked to the surface to create the inner leaflet, promoted 
by the amphiphilic nature of the interface. Phospholipids are self-assembled around the exterior. This object maintains a $\mathrm{pH}$ gradient across the lipid coating for several days..... 12

Figure 1.17. A pH sensitive hydrogel modified by covalently linking palmitolyl chloride to the surface. When a $\mathrm{pH}=12$ buffer was flowed into the channel (a), the modified $\mu$ gel remained stable for hours without obvious signs of change while the unmodified $\mu$ gel of the same size and composition started to swell instantly (b). After physically disrupting the fatty acid layer by piercing the $\mu$ gel with a micropipette tip (c), the modified $\mu$ gel began to expand at the disrupted location $(\mathrm{d}, \mathrm{e})$ and eventually became fully swollen accompanied by the phenolphthalein indicator's color change (f). Scale bars are $100 \mu \mathrm{m}$.

Figure 1.18. In contrast to an unmodified $\mu$ gel ( $\boldsymbol{\square})$, a $\mu$ gel covalently modified (a) with fatty acids ( ) doesn't expand in $\mathrm{pH} 12$ buffer solution (b) until SDS was added to the buffer. The expansion quickly began at points on the $\mu$ gel surface (c), which propagated (d-g) until

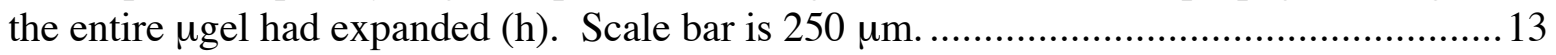

Figure 1.19. Plot of the mean expansion time for $2 / 3 \mathrm{~F}_{\mathrm{D}}$ as a function of the surfactant concentration

Figure 1.20. Phospholipids are self-assembled around the modified $\mu$ gel to form the second leaflet of the bilayer.

Figure 1.21. The upper block of images corresponds to confocal images of a phospholipid layer assembled on a fatty acid modified $\mu$ gel while in water. The lower block of images is the same sample imaged under the same parameters immediately after a $10 \mathrm{mM} \mathrm{Na} \mathrm{s}_{2} \mathrm{~S}_{2} \mathrm{O}_{4}(\mathrm{Ph}$ 11) quenching solution was introduced to the channel. The decrease in fluorescence intensity in the lower block of images is due to the interaction of the ionic quencher with the fluorophores attached to the head groups of phospholipids at the lipid/ water interface...... 16

Figure 1.22. A hydrophilic spacer (a) is attached to the surface of the $\mu$ gel to allow for the incorporation of transmembrane proteins. Then vesicles formed with ITC-lipids (b) are fused to the $\mu$ gel. Reaction of the ITC moiety with the amines presented by the tether would result in the formation of a covalent urethane linkage. 17

Figure 1.23. Lipid bilayer formation should proceed with one step. After the gly-gly tether attached to the hydrogel is deprotected to yield the free amine $(a, i)$, the $\mu$ gel is incubated with vesicles (aq) that were made with some ratio of isothiocyanate-functionalized phospholipids (ii). Since the reaction between an amine and an isothiocyanate to create a covalent urethane linkage is reported to occur within minutes in the presence of water, this reaction should occur simultaneously to the vesicle fusion process, ultimately creating a phospholipid bilayer covalently tethered in some locations to the surface of the $\mu$ gel (b, iii).

Figure1. 24. Representative histograms of the frequency of half-life values for $\mathrm{pH}$-sensitive $\mu$ gels modified with saturated n-alkyl fatty acids. The number of samples with a half-life within the specified time interval (i.e., $0-5 \mathrm{~h}$ ) is indicated in each column

Figure 1.25. Confocal images of 12 hydrogel posts oxidized at several concentrations (10, 50, and $\left.100 \mathrm{mM} \mathrm{NaIO}_{4}\right)$ and different temperatures $\left(0,23,50,80{ }^{\circ} \mathrm{C}\right)$ for 10 minutes (a). A graph showing the hydrogel oxidation depth (average value of each side of the hydrogel) as a function of temperature (b). These results indicate that oxidation depth increases with increasing temperature and concentration. 
Figure 1.26. 2-Aminooxy-N-decyl-acetamide modified hydrogels pre-soaked in a $\mathrm{pH}=7.25$ buffer. Addition of a $0.1 \mathrm{mM}$ SDS / $\mathrm{pH}=7.25$ buffer induced a breakdown of the chemical barrier produced by the chemisorbed alkyl chains. Swelling began slowly then once break point was introduced (see $215 \mathrm{~min}$ ) the swelling increased quickly.

Figure 1.27. The frequency of the half-life values for the ion barrier stability of $\mathrm{pH}$-responsive hydrogels modified with acid chlorides composed of an even number of carbons (2-16), all with saturated alkyl chains. The samples that had a half-life value within the specified $5 \mathrm{~h}$ interval (i. e. 0-5h) are indicated with each column. Fifty samples were tested for each acid chloride.

Figure 1.28. White and parallel fluorescent images of S-S crosslinked post taken during different exposure time to lysed TCEP-HCl encapsulated liposomes. Post with unlysed liposomes $(\mathrm{a}, \mathrm{d})$, one minute after introduction of lysed liposomes (b, e), and after ten minutes of exposure $(c, f)$. The exposure to released TCEP-HCl leads to dissolution of the post as seen by difficulty in edge detection and decrease in post size (c, e, f). Liposomes contain a trace amount of fluorophore (rhodamine)-conjugated lipids on its surface for detection. The initial post is $\sim 500 \mu \mathrm{m}$ in diameter.

Figure 1.29. Edge and downstream of the liposome-capturing filter before and after introduction of complement serum into the microchannel. Complement cascade triggered liposome lysis and unloading of the florescent (CF, carboxyfluorescein) cargo. Such lysis is indication of specific biochemical detection event. The blue square in the inset shows the area in the channel where the micrographs were taken. 24

Figure 1.30. The time scale necessary for complement cascade to lyse the activated liposome is examined. Close to $90 \%$ reaction completion is achieved within 15 minutes....................25

Figure 1.31. Phase-transfer reaction through a Nylon-6,6 membrane. (a) Control reaction with no PTC in xylenes phase, no red anion observed. Phase-transfer reaction through Nylon 6,6 membrane at time $=0$ (b) and at (c) 120 seconds.

Figure 1.32. Chelating metal extration. A control reaction with no $\mathrm{Pb}(\mathrm{II})$ ions in water time $=$ $5 \mathrm{~min}(\mathrm{a})$. Chelating metal extraction time at 1 (b), 3 (c), and 5 (d) minutes respectively. ..27

Figure 1.33. A schematic diagram (a) of con-current flow is shown for the top row of figures which show con-current extraction of $\mathrm{Co}$ (II) (blue) being extracted from the aqueous phase(lower) to the organic phase (upper). A schematic diagram (b) of counter-current flow is shown for the bottom row of figures that show counter-current extraction. ..................28

Figure 1.34. Synthesis of biologically active membranes. a) Schematic diagram of a photopatterned H-channel. The shaded area is hydrophilic and was exposed to light. The remainder of the $\mathrm{H}$-channel was not exposed to light and remained hydrophobic. b) a close up view of the highlighted box in (a). The hydrophilic side of the channel was filled with an aqueous solution containing the peptide monomer. A toluene solution containing adiptoyl chloride filled the hydrophobic side of the channel. c) a micrograph of the highlighted section of the channel, after $10 \mathrm{~min}$ of interfacial polymerization. d) a micrograph of the membrane after 30 min of interfacial polymerization, rinsing $(\mathrm{MeOH})$ and drying under $\mathrm{N}_{2}$.

Figure 1.35. Model reaction and visualization using dyes and fluorophores. a) Ehylene diamine reaction with hydrogel produces an amine functional hydrogel. Visualization of primary amine functionality with bromophenol blue dye. b) Confocal image of a model reaction of 
isothiocyanate groups with the amine functional hydrogel, fluorescent dye $=$ fluorescein isothiocyanate. c) Confocal image of a model reaction of amine groups with isothiocyanate functional hydrogel, fluorescent dye = Lucifer Yellow...................................................... 31

Figure 1.36. A photopolymerized hydrogel using the model crosslinker obtained from Scheme 1 .

Figure 1.37. The dissolving curve of hydrogels (a) with different original diameter. All experiments were carried out using $50 \mathrm{mM}$ DTT solution in $\mathrm{pH} 7.4$ Tris buffer at a flow rate of $0.2 \mathrm{~mL} / \mathrm{min}$. (b) An acrylimide hydrogel crosslinked via a disulfide linkage. 32

Figure 1.38. Estrogen-responsive hydrogel. The interaction of the dimerized estrogen receptor bound to immobilized ligand forms the active, noncovalent crosslinks. A toxin (EDC) will compete for the ER's binding pocket, releasing the ER from the tethered ligand, and breaking the active crosslinks of the hydrogel.................................................................... 33

Figure 1.39. Estrogen-responsive hydrogel. Estradiol is conjugated to a photopolymerizable tether, and allowed to bind to the ER, enhancing ER dimerization. The hydrogel is polymerized around this noncovalent crosslinker. 34

Figure 1.40. The effects of several monomers on ER activity, determined by the HAP assay (a), and the effects of two crosslinkers on ER-ES2 binding, determined by the HAP assay (b). 35

Figure 1.41. Optimization of acrylamide concentration. Hydrogels were polymerized with a $400 \mu \mathrm{m}$ diameter circle photomask. Monomer solutions with 15\% AAm formed gels with good integrity while 10\% AAm gels were unstable and 5\% AAm gels did not polymerize.36

Figure 1.42. Optimization of NMBA (inert corsslinker) concentration. Hydrogels were polymerized with a $400 \mu \mathrm{m}$ diameter circle photomask. Hydrogels with $1 \%$ NMBA contained the lowest degree of crosslinker needed to form stable gels while $0.3 \%$ and $0.7 \%$ NMBA gels were too soft to be functional. 36

Figure 1.43. Optimization of polymerization time. The gels contained: $15 \% \mathrm{w} / \mathrm{w}$ AAm to buffer, $1 \% \mathrm{NMBA}$ to AAm, $1.2 \% \mathrm{w} / \mathrm{v} \mathrm{BP}^{+}$to solution, and 1.2\% w/v NMDA to solution. Polymerization times less than $1 \mathrm{~min} 45 \mathrm{sec}$ resulted in smaller gels (incomplete polymerization) while times greater that $1 \mathrm{~min} 45 \mathrm{sec}$ resulted in larger gels (over polymerization) when compared to the $400 \mu$ m photomask.

Figure 1.44. Model study of disulfide crosslinked poly(acrylamide) hydrogel. Test mixtures contained $7 \% \mathrm{w} / \mathrm{w}$ CBA to AAm and 1\% w/w NMBA to AAm. Control 1 contained $7 \%$ w/w NMBA to AAm. Control 2 contained 1\% NMBA w/w to AAm crosslinks. (a) Response of disulfide crosslinked hydrogel to DTT showed the $f_{d}=$ (final diameter-initial diameter)/initial diameter. (b) Degree of swelling of disulfide crosslinked hydrogel upon introduction of DTT.

Figure 1.45. Fluorescence polarization studies were performed on serial dilutions of the competitor to determine its ability to bind to the estrogen receptor. A decrease in millipolarization $(\mathrm{mP})$ indicates that binding occurred.

Figure 1.46. A hydrogel is shown crosslinked with a peptide sequence cleavable by a specific protease.

Figure 1.47. A polymerizable crosslinker synthesized and conjugated to the tetrapeptide through its terminal cycteines.

Figure 1.48. Digestion of the tetrapeptide by $\alpha$-chymotrypsin. The tetrapeptide $(\mathbf{A}, 2.0 \mathrm{mg})$ was dissolved in PBS (1 mL, pH 7.4). Some of the peptide oxidized into the cyclic form, B. $\alpha$ - 
Chymotrypsin $(0.2 \mathrm{mg})$ was added to the peptide solution and HPLC spectra were taken after 0, 5, and 30 min. Peptide peaks $\mathbf{A}$ and $\mathbf{B}$ disappeared with time after the addition of the protease. The digested fragment, $\mathbf{C}$, was more polar than the intact peptide and eluted with the solvent front. Its intensity increased with time.

Figure 1.49. Optical micrographs of hydrogels with 5, 10, and $18 \%$ surfactant (Brij 58) after shrinking ( $\mathrm{pH} 3.02)$ and swelling $(\mathrm{pH} 10.85)$.

Figure 1.50. The effect of surfactant concentration on the oxidation depth of a $\mathrm{pH}$ responsive hydrogel containing glycerol monomethacrylate and acrylic acid. The diol moieties of hydrogels containing different concentrations of surfactant (Brij 58) and water were oxidized with $\mathrm{NaIO}_{4}$ and conjugated to Lucifer yellow to determine the degree of oxidation. 1) $0 \%$ Brij 58, $0 \%$ water 2) $10 \%$ Brij 58, $0 \%$ water 3) $18 \%$ Brij 58, $0 \%$ water, 4) $0 \%$ Brij $58,100 \%$ water 5) $0 \%$ Brij 58, $200 \%$ water 6) $18 \%$ Brij 58, $100 \%$ water 7) $18 \%$ Brij 58, $200 \%$ water a) $10 \mathrm{mM} \mathrm{NaIO}_{4}, 10$ min oxidation, b) $10 \mathrm{mM} \mathrm{NaIO}_{4}, 50$ min oxidation, c) 50 $\mathrm{mM} \mathrm{NaIO}_{4}, 10$ min oxidation, d) $50 \mathrm{mM} \mathrm{NaIO}_{4}, 50$ min oxidation.

Figure 1.51. Multifunctional biotin ligands. Biotin dimers with a) a poly(ethylene glycol) linker and b) an aliphatic linker. c) Biotin trimer with a poly(ethylene glycol) linker.......45

Figure 2.1. Concept photo depicting rapid channel fabrication in its simplest form.................46 Figure 2.2. Schematic (a) of self-sealing, alignment free $\mu$ fluidic fabrication. Resultant channels (b) are shown using the glass sandwiching technique. The channels were flushed with $\mathrm{MeOH}$ and filled with dyed water.

Figure 2.3. a) An example of a universal cartridge showing three separate channels, and b) a channel network inside the universal cartridge with press-on connectors is shown. c) A multiplayer device showing interconnected layers. 48

Figure 2.4. Conceptual drawing of interconnected microfluidic device. The different colors represent components needed for the integrated biodetection device. 48

Figure 2.5. A schematic (a) is shown of a three- layer device. Layers 1 and 3 are connected through layer 2 but do not interact with the channel formed in layer 2. Dyed water is shown flowing through the device and as the blue and yellow dyed water come together in the chaotic micromixer, the fluid streams turn green (b). The red channel flows through the device but is not connected to the other two layers (c). Channel widths are 800 microns. .. 48

Figure 2.6. Channel walls patterned in PEG, (a) $175 \mu \mathrm{m}$ width, and (b) $1,000 \mu \mathrm{m}$ width. An accuracy of less than $10 \mu \mathrm{m}$ was achieved with both channel widths.

Figure 2.7. Photopatterned valve substrates, (a) made with PEG, and (b) with poly (IBA). The comparison shows features are similar in dimension but features are rounded with PEG but more optimization is needed.

Figure 2.8. FTIR spectra of photopolymerized PEGdA, (a) with $0.1 \mathrm{wt} \%$ photoinitiator and (b) with $0.05 \mathrm{wt} \%$ photoinitiator. The amount of carbon-carbon double bonds decreases with increasing photoinitiator showing the increased polymerization of the PEGdA. A balance between concentration of photoinitiator and free monomer after polymerization will maximize biocompatibility.

Figure 2.9. Two hydrogel walls were polymerized from mixtures with different breakable crosslinkers, while a hydrogel post was polymerized from a mixture with non-breakable crosslinker (a). One of the hydrogel walls dissolved completely in the presence of $5 \%$ 
$\mathrm{NaIO}_{4}$ solution at a flow rate of $0.1 \mathrm{~mL} / \mathrm{min}$, while the other hydrogels were not affected (b). The other hydrogel wall was dissolved using $10 \mathrm{mM}$ TCEP solution at a flow rate of 0.1 $\mathrm{mL} / \mathrm{min}$ (c). The hydrogel post in the center was not affected. Bars shown are $500 \mu \mathrm{m}$.

Figure 2.10. Schemes of multiphase liquid laminar flows and corresponding images of aqueous liquid flow inside channels after surface patterning. The liquid is a dilute solution of Rhodamine B dye (conc. $0.057 \mathrm{w} / \mathrm{w} \%$ ) in deionized water.

Figure 2.11. Water confined by a virtual wall under a) $12 \mathrm{~mm}$ of water pressure and b) $5 \mathrm{~mm}$ of water pressure.

Figure 2.12. Solution on top is $1 \mathrm{M} \mathrm{KI}$, on bottom is concentrated nitric acid. a) residual water in system mixes with nitric acid to create steam (whitish). b) wall starts to give out. c) redox reaction ensues.

Figure 2.13. Schematic (inset) shows an example of the active assembly method applied to filter fabrication. The white area between the top and bottom channels in the picture (a) is an in situ fabricated filter. The washing flow comes from left to right while not passing through the fabricated filter during the cleansing process. Once the filter is fabricated, the blocking components (b) located in the top left and bottom right side of (a) blocks the flow causing the sample solution to flow in the direction indicated by the curved arrow as shown in

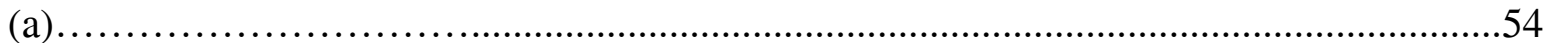

Figure 2.14. Fabrication of the biomimetic hydrogel check valve. A. After simultaneous polymerization of the $\mathrm{pH}$ sensitive hydrogel srips $\mathrm{b}$. after polymerization of the non $\mathrm{pH}$ sensitive strips to for the bi=srip hydrogel valves with anchors. C. When exposed to basic solution,k the bistrip hydrogel expands and curves to form a normally closed vlave. D. when exposed to ascidic solutions, the valve is deactiveate, returning to the permantly open state scale bars $500 \mu \mathrm{m}$..

55

Figure 2.15. Pressure profile upon forward flow. After staring fluid flow at $1.0 \mathrm{~mL} / \mathrm{min}$, the pressure increases reapidly. When the pressure reaches $1.5 \mathrm{psi}$, the valve opens to allow forward fluid flow. Upon stopping the flow, the valve gradually closes. ..........................56

Figure 2.16. Formation of microchannel and valve. a) Step 1: formation of channel via URP process. b) Step 2: flow in construction material and polymerize valve anchor. c) Step 3: Flow in responsive hydrogel material and polymerize valve. .56

Figure 2.17. a) A multichannel device shown with different colored dyes and check valves that prevent backflow. b) The construction of multiple non-responsive valve anchors in a microchannel, and c) the addition of the responsive component of the valves is

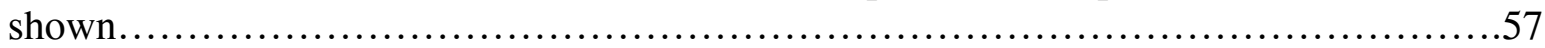

Figure 2.18. Schematic representation of the hydrogel valve ...........................................57

Figure 2.19. Hydrogel check-valve before (a) and after swelling (b).....................................58

Figure 2.20. The operating pressure cureve of the hydrogel valve is shown for a forward flow

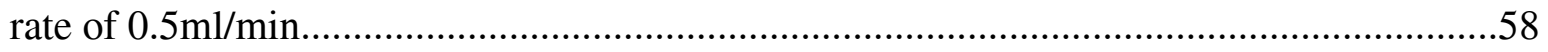

Figure 2.21. SEM images of a filter made with different initiator concentration. a) $0.378 \%$ b) $1 \%$

c) $1.5 \%$ d) $2 \%$ initiator. Scale bar is slightly different and is shown for each image. .......60

Figure 2.22. Two examples of Confocal micrographs showing the pore size and distribution of the polyHEMA beads in the filter. The scale is shown within the images ........................61

Figure 2.23. Resistance of the filters in a microchannel. ...............................................61 
Figure 2.24. Confocal micrographs of the 2 filters studied. F1 was prepared from $27 \%$ monomer, $2.3 \%$ cross-linker and $1 \%$ photo-initiator. F2 was prepared $27 \%$ monomer, $3.3 \%$ cross-linker and $1 \%$ photo-initiator.

Figure 2.25. Separation of blood cells from rabbit whole blood. (a) After filter (b) Before filter. The black dots are the red blood cells while the white dots are white blood cells. The cell size is around $6 \mu \mathrm{m}$ in diameter.

Figure 2.26. Kinetics of glucose-6-phosphate dehydrogenase enzyme.............................63

Figure 2.27. A top view and a side view of a two-layer device used to place the filter inline with the fluid path. Two different size beads ( 3 micron and 45 micron) were introduced into the device using a 5 micron pore size filter.

Figure 2.28. A closeup view (white circle indicates the outer filter edge) of the device is shown with beads flowing through (a) initially (b) with darkfield on and (c) after a few seconds at a flowrate of $10 \mathrm{ml} / \mathrm{min}$. A photograph (d) of the device is shown which shows the 3 micron beads (pink) in the filter and the shadow outline of the entire filter.....................64

Figure 2.29. The decrease in intensity of the PP-gel for different flow rates...........................66

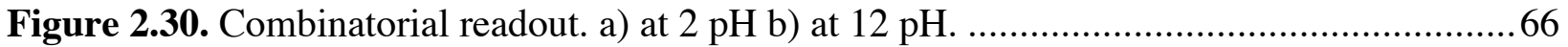

Figure 2.31. A confocal study of diffused protein (FITC-BSA) ring(a) around hydrogel. A micrograph of a $\mathrm{pH}$ responsive hydrogel (b) with protein molecules embedded in the hydrogel matrix. Gray-scale intensity profile (c) of fluorescent protein content in a hydrogel.

Figure 2.32. A channel (a) showing hydrogel posts before exposure and hydrogel posts with $5 \%$ BSA (b) in $0.05 \mathrm{M}$ phosphate buffer.

Figure 2.33. A sideview of an acryl amide gel containing biotinylated $\mathrm{IgG}$, after exposure to Extravidin/Alkaline phosphatase conjugate and p-nitrophenyl phosphate. .68

Figure 2.34. (a) Hydrogel posts are formed using a polycarbonate top with a gasket height of $125 \mu \mathrm{m}$. The polycarbonate top and gasket are removed, and a new polycarbonate top with a gasket height of $250 \mu \mathrm{m}$ is put in place. Channels are formed around the gels. (b) Two inlet holes and one outlet hole are punched in the polycarbonate top and channels are formed. The hydrogel monomer is flowed in through first inlet well, and water is flowed in through the second inlet well, displacing the acryl amide monomer solution. UV light is used to polymerize the gel, and the channel is flushed, leaving a short gel on the bottom of the channel

Figure 2.35. The diameters of all of gels with different amounts of crosslinker were measured. Results indicate that gel-swelling can be controlled by increasing the cross-linker concentration.

Figure 2.36. A top view shows $\sim 20 \mu \mathrm{m}$ tall reactive posts patterned by polymerizing Avidin in acryl amide gels. Channels were formed around the posts to direct reagent flow. An ELISA was performed, using biotinylated rabbit-anti-botulinum $\mathrm{E}$ as primary antibody, and ultimately produced a visible signal.

Figure 2.37. Side view shows the top of the gel does not impinge upon the top of channel. This provides maximum exposure of the gel to reagents, increased surface area, and intensification of signal to the observer.

Figure 2.38. Agarose gel as the readout 5 min after introducing the substrate. The channel with is $1000 \mu \mathrm{m}$. 70 
Figure 2.39. Progression of virtual wall breakdown with a hydrogel using a $\mathrm{pH}$ indicator phenolphthalein and a base. (a) Virtual wall separating two fluids, one with a hydrogel arm present. The top fluid is basic, and the bottom fluid has indicator in it. (b) The hydrogel begins to grow towards the other solution across the virtual wall. (c) The hydrogel is shown just before complete virtual wall breakdown. (d) Virtual wall breakdown is shown with pink color from the mixing of $\mathrm{pH}$ indicator and base.

Figure 2.40. Schematic of the microfluidic device. Shaded portion denote the location of the polymerized emulsion filter. Clear circles downstream indicate normal acrylamide gel posts, while gray circles denote disulfide crosslinked posts. Arrow indicates the direction of flow. Posts are about $500 \mu \mathrm{m}$, and the width of the horizontal channel is $3000 \mu \mathrm{m} . \ldots \ldots \ldots . .72$

Figure 2.41. Filter with TCEP-HCl encapsulated liposomes and downstream acrylamide gel posts in the channel. Upstream filter seen in white light (a) and in fluorescence (b) where large clumps of vesicles are visible within the filter and on upstream surface. With $10 \%$ triton, vesicles are solubilized (c), unloading encapsulated TCEP-HCl downstream. Downstream, acrylamide gel posts with disulfide crosslinkers slowly dissolve (d, outer two posts are non-dissolving, only the center post contains S-S crosslinkers) and a tether is formed ( $\mathrm{d}$ and e, arrows) in direction of the flow (e). Direction of flow is right to left. .....72

Figure 2.42. Effect of liposome released TCEP-HCl on normal acrylamide post (a) and on disulfide crosslinked posts $(b, c)$ in a microfluidic channel. Posts a and b were next to each other close to the filter, while post $\mathrm{c}$ was located downstream. A significant uptake of fluorophore-labeled lipid is seen (b3) which indicate reduction of disulfide crosslinks and dissolution of the gel post. The S-S crosslinked post located further downstream show reduced uptake of fluorescent lipid, which penetrated only the outer edges of the post (c3).

Figure 2.43. A schematic is shown of a 3D serpentine chaotic mixer (a). Layers 1 and 2 are connected to form a micromixer with blue and yellow dye. The streams start to appear green by the first turn in the channel (b).

Figure 2.44. A magnetic mixing device is made by positioning a blade inside a cavity, filling the cavity with prepolymer, and exposing the device to UV to form the channel network (a). A $275 \mu \mathrm{m}$ mask is placed over the mixing chamber (b), and a post is polymerized inside the hole of the blade (c). A completed device is shown (d). 74

Figure 2.45. The $2.5 \mathrm{~mm}$ device, shown before mixing begins (a), during mixing at a flow rate of $2 \mathrm{~mL} / \mathrm{min}$ (b) and at a flow rate too high $(8 \mathrm{~mL} / \mathrm{min}$ ) for the mixer to mix efficiently (c). Channel width is approximately $1 \mathrm{~mm}$. 75

Figure 2.46. Sequential images of dye solution (1\% wt. Brilliant Green in $\mathrm{MeOH}$ ) filling the $\mathrm{H}-$ channel. This membrane was exposed to a buffer solution not containing chymotrypsin. The channel was initially dry and empty prior to filling. Notice the directed filling by the membrane, indicating no change in the membrane porosity. 76

Figure 2.47. Sequential images of dye solution (1\% wt. Brilliant Green in $\mathrm{MeOH}$ ) filling the $\mathrm{H}-$ channel. This membrane was exposed to a buffer solution containing chymotrypsin. The channel was initially dry and empty prior to filling. Notice the solution permeating though the membrane indicating a change in the membrane porosity. .................................. 76

Figure 2.48. Sequential images of a membrane completely dissolving at room temperature.....77 
Figure 2.49. a) A schematic (side view) of a fluid pushing design and b) a top view of $300 \mu \mathrm{m}$ hydrogel posts polymerized in the actuation chamber of the device.............................78

Figure 2.50. Schematic representation of the thumb actuated pump (TAP).........................79

Figure 2.51. Passive pumping. The surface tension of the green drop in (a) provides enough force to cause the drop to move into the microchannel (b) without any external effort. As long as there is a larger "reservoir" drop somewhere in the microfluidic system, fluid can be pumped from any number of ports to the reservoir drop. 80

Figure 2.52. Mechanical model of the micro pump. The disk actuator is driven by an external DC motor through magnetic coupling.

Figure 2.53. Rotary pump built in a two-layer microtectonics platform. The actuator consists of a ferromagnetic bar embedded in a polymer disc, placed in the first layer.

Figure 2.54. Diagram showing the path flows in (a) closed cylinder with only an opening at the top (b) cylinder with an opening at the top and another one in the left part (micropump). A non-pulsating net flow is created due to viscous drag, between layers of the fluid and adhesion to the surface of the rotating disk. 82

Figure 2.55. Flow rate versus frequency of rotation of the disc pump and of an impeller pump. 82 Figure 2.56. Velocity 'V'and max. shear stress ' ${ }_{\text {max }}$ ' in (a) $\mu$ pump (b) impeller in a big flask. In case (a) assuming that $\mathrm{l}=\mathrm{d} / 2=0.275 \mathrm{~mm} / 2$, with a frequency of rotation $\mathrm{f}=10 \mathrm{~Hz}$, then ${ }_{-\max }$ $=5.6 \mathrm{dynes} / \mathrm{cm}^{2}$. In case (b) the maximum gradient of $\mathrm{V}$ is in the $\mathrm{x}$ direction, which means that ${ }_{\text {max }}$ is only function of the frequency of rotation. 83

Figure 2.57. EVA tubing with construction material coiled inside (a) and after being removed from the tubing (b). An SEM image of the coil (c) reveals a sinusoidal shaped structure. . 84

Figure 2.58. EVA tubing formed around the responsive hydrogel material inside (a). The responsive hydrogel in basic solution initially (b) and after 30 minutes (c)...................8 84

Figure 2.59. The EVA tubing is cut into pieces (a) and heated on top of a piece of glass (b), to form relatively straight pieces of responsive hydrogel material (c). 85

Figure 2.60. The threads were soaked in an acidic solution and woven into a 6 by 6 grid (a), allowed to dry out (b), re-hydrated with solution (c), and then exposed to base and allowed to swell for 30 minutes $(\mathrm{d})$. .85

Figure 3.1 . A comparison (a) is shown of experimental and numerical swelling of a $300 \mu \mathrm{m}$ hydrogel. A similar comparison (b) is shown of experimental and numerical deswelling of $300 \mu \mathrm{m}$ hydrogel.

Figure 3.2. Swelling (a) and deswelling (b) of $150 \mu \mathrm{m}$ gel shows good agreement between the numerical and experimental data.

Figure 3.3 Hydrogel deformation model......................................................................... 88

Figure 3.4. a) Swelling of 500 and 700 micron gels from $\mathrm{pH}=2$ to 12 , and b) forces exerted by the gel on top and bottom surfaces during swelling ................................................... 88

Figure 3.5. Variation of the profile of a biofuse for various outside concentration of DTT (a). and variation of the profile of a biofuse for various crosslinking density (b)...................90

Figure 3.6. Variation of the profile of a biofuse for varying temperature (a). Variation of the oxidation depth with outside concentration is shown for a fixed time of 50 minutes (b)....90

Figure 3.7. Swelling of 150-micron gel (a) and Deswelling of 150-micron gel (b) show good agreement with experimental data. 
Figure 3.8. Deformation profile in the gel-bath system during swelling (a) and swelling of the gel for a $10 \mathrm{~V}$ electric field (b).

Figure 3.9. Young's modulus as a function of the $\% \mathrm{w} / \mathrm{w}$ of cross-linker in the hydrogel chemistry. Each data point represents the average of five samples while the error bars denote the standard deviation.

Figure 3.10. The response of an E-gel towards a voltage signal of amplitude $12 \mathrm{~V}$ with an offset

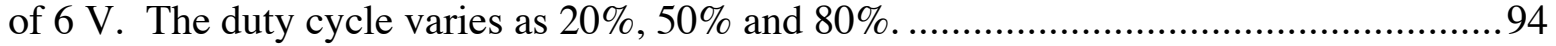

Figure 3.11. Variation in the hydration state..........................................................94

Figure 3.12. Cross-sectional schematic of the cantilever/polymer structure with the various dimensions.

Figure 3.13. This figure shows the comparison of the simulation results with the experimental results. It is to be noted that the tip of the beam touches the bottom surface of the cantilever well before the beam can bend fully. Thus the motion of the beam is restrained. However, due to the slow response of the $\mathrm{pH}$ responsive hydrogels, hybrid actuation (simultaneous stimulus of $\mathrm{pH}$ and applied electric field) can be done to improve the response speed of the device acting as a transducer. .96

Figure 3.14. This figure shows the variation in the equilibrium bending of the micro-sized composite cantilever beam with varying length of the beam ....................................... 96

Figure 3.15. This figure shows the relation of tip deflection to the thickness of the gel only....97

Figure 3.16. The comparison of tip deflection obtained for various hybrid actuations.............97

Figure 3.17. Kinetic analysis shows that the sensor can have a better speed of response in the presence of an applied field 98

Figure 4.1 Tension sample of construction material used to determine mechanical behavior and material properties.

Figure 4.2. The stress-strain relationship of three hydrogel samples with displacement rates of $1 \mathrm{~mm} / \mathrm{min}, 2 \mathrm{~mm} / \mathrm{min}$ and $4 \mathrm{~mm} / \mathrm{min}$. The graph shows a stress-strain relationship characteristic of a semi-crystalline polymer. 100

Figure 4.3. Images of the responsive hydrogel undergoing deformation. ........................... 100

Figure 4.4. - The Instron Micromechanical Testing Machine (a). An EPON patterned silicon wafer (b) was used to mold PDMS to produce a thin stencil (c). Responsive hydrogel was injected into the stencil and polymerized to form well-defined tensile samples. 101

Figure 4.5. The ultimate tensile strength shows a decrease from $250 \mathrm{kPa}$ to $50 \mathrm{kPa}$ as $\mathrm{pH}$ is

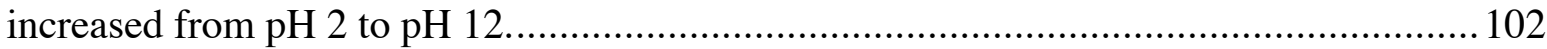

Figure 4.6. The percent elongation at break decreases from $140 \%$ at $\mathrm{pH} 2$ to $30 \%$ at $\mathrm{pH} 12 \ldots 102$

Figure 4.7. Expansion in $\mathrm{pH} 12 \mathrm{NaOH}$ solutions with various quantities of $\mathrm{NaCl}$ added......... 103

Figure 4.8. Contraction in $\mathrm{pH} 2 \mathrm{HCl}$ solutions with various quantities of $\mathrm{NaCl}$ added. .......... 103

Figure 4.9. Optical image (side view) of a hydrogel used in force response tests. The cylinder

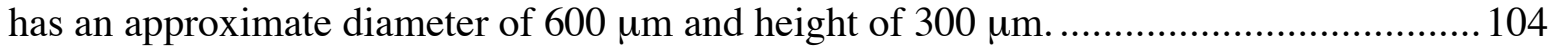

Figure 4.10. Typical force response curve generated by a $600 \mu \mathrm{m}$ hydrogel structure swelling in $\mathrm{pH} 12$ buffer. The hydrogel exerted a maximum force of $0.14 \mathrm{~N}$ on the upper platen of the test machine after approximately 2.1 hours of swelling. 104

Figure 4.11. Young's modulus as a function of the $\% \mathrm{w} / \mathrm{w}$ of cross-linker in the hydrogel chemistry. Each data point represents the average of five samples while the error bars denote the standard deviation. 105 
Figure 4.12. Series of time lapsed (1 min intervals) pictures of a partial hydrogel array after being exposed to $\mathrm{pH} 12$.

Figure 4.13. The maximum load exerted by an array of 40 gels plotted versus their respective volumes. 106

Figure 4.14. The variation of Young's modulus with weight percent crosslinker is shown (a). The standard hydrogel chemistry used in the BioFLIPS designs incorporates $1 \%$ crosslinker. Each data point represents 5 samples. (b) The impact of both crosslinker variation and acrylic acid variation are shown for values near the standard hydrogel chemistry (1 wt.\% crosslinker, 20 molar\% AA). 107

Figure 4.15. The impact of molar percent acrylic acid on the elastic modulus of standard hydrogel chemistry. 108

Figure 4.16. Side view of sample chamber (a) and front view of sample chamber and loading platform (b) designed for force response testing. The platen attached to the load cell at the top of the figure is held at a fixed displacement. The cylindrical hydrogel sample is polymerized in situ using UV irradiation from below. Solutions of controlled $\mathrm{pH}$ level are circulated in the chamber during testing. 110

Figure 4.17. Graph of the Young's Modulus with respect to curing temperature for PDMS with a 10:1 and 11:1 weight ratio of prepolymer to hardener. 110

Figure 4.18. Affect of membrane stiffness on PDMS membrane valve operation. Cross section image (a) and schematic (b) of a hydrogel membrane valve cured at $70^{\circ} \mathrm{C}$. (c) Schematic cross section of a membrane valve cured at $85^{\circ} \mathrm{C}$. The effects of aging may cause a valve to function normally initially, but fail due to shelf life issues later. The scale bar corresponds to $100 \mu \mathrm{m}$.

Figure 4.19. Schematic showing the laminar flow of chemicals that are used to define the hydrophobic and hydrophilic regions of the channel. The gray regions become hydrophobic after the chemicals are removed (a). A schematic three-dimensional representation of the fluid flow in the channel (b).

Figure 4.20. Velocity profiles (a) in the $x-z$ plane are shown (looking down at the top of the channel as shown in Figure 4.19b). The curves are the velocity profiles at various depths (y positions). Reconstruction of the flow (b) in the $x-y$ plane from the centerline velocities of the curves as shown in (a).....

Figure 4.21. Calculated velocity vectors superimposed on an image of the fluorescent particles moving near the channel input. The image has been altered (black regions converted to white, and vice versa) to more clearly show the air-water interface.

Figure 4.22. A pH-sensitive hydrogel was fabricated between two silver wires. The first image is of the water-swollen hydrogel before any voltage was applied. The second image shows the hydrogel 5 minutes after a $3 \mathrm{~V}$ potential was introduced. The scale bar is approximately $1 \mathrm{~mm}$. 113

Figure 4.23. The prototype on the left utilizes the cartridge fabrication process to create microfluidic channels. The image on the right shows hydrogels as they appear in the finished device.

Figure 4.24. Hydrogel response curves; measured time constants are expressed in seconds next to each response. 115 
Figure 4.25. Typical hydrogel response; the response begins towards the anode and spreads across the remainder of the hydrogel. A scale bar has been added to highlight the difference in jacket thickness before and after turning the voltage on. 116

Figure 4.26. Graph of hydrogel thickness with respect to time. The duty cycle was changed as indicated on the graph. The inset pictures correspond to a $20 \%$ duty cycle and $80 \%$ duty cycle respectively. The bar on the hydrogel measures $400 \mu \mathrm{m}$. 117

Figure 5.1. Detailed drawing of the actuation chamber, hydrogel, and star orifice. a. Off axis side view, b. top view, c. side view of a device operating at $25 \mu \mathrm{L} / \mathrm{min} \mathrm{pH} 2$ buffer flow, d. off axis side view, e. top view, and f. side view of a device operating at $100 \mathrm{uL} / \mathrm{min} \mathrm{pH} 2$ buffer flow rate. The $\mathrm{pH} 2$ buffer is shown in blue, and the $\mathrm{pH} 12$ buffer is shown in pink. Observe the size of the hydrogel post and the resulting imprint over the star orifice at the two given flow rates. The interface between $\mathrm{pH} 2$ buffer and $\mathrm{pH} 12$ buffer also changes with different flow rates.

Figure 5.2. Graphs showing a, Flow rate of $\mathrm{pH} 2$ buffer vs. time. b, $\mathrm{pH}$ of the output stream vs time for two separate devices, one with a hydrogel sensor/actuator and one without. The two pictures show the hydrogel sensor/actuator at two separate flow rates. The arrows point to when the picture was taken during the experiment. When the $\mathrm{pH} 2$ flow is increased, the hydrogel senses this and shrinks to allow more $\mathrm{pH} 12$ buffer through the star valve to linearize the $\mathrm{pH}$ 120

Figure 5.3. Parallel BoNT bio-detection using both an ELISA assay and bio-responsive materials. 121

Figure 5.4. Layout of the ELISA device. All fluidic paths are filled with food color for visualization For protein storage and driving flow through the channel network, 6 TAPs were included. The TAP is micro-molded from PDMS and consists of a reservoir, an outlet port and an adhesive layer at the bottom (inset A). A chaotic mixer was fabricated between the 2 layers of the device. Fluid moving through the chaotic mixer when TAP is depressed is shown in inset B. A porous hydrogel was utilized to separate blood cells from whole blood (inset $\mathrm{C}$ ). To prevent contamination between the reagents and backflow, 4 checkvalves were fabricated in the device. Each valve consists of a rigid post and a hydrogel piston (inset D). The direction of flow allowed is indicated as arrows in the layout. The readout consists of filter membrane and agarose beads. ELISA is performed on (and in) the gel beads. The color of the beads after the assay is shown in inset $\mathrm{E}$ 124 


\section{Table of Tables}

Table 1.1. Concentration of hydrogel components resulting in 50\% ER activity...................35

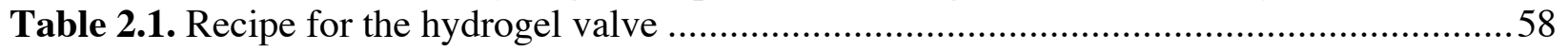

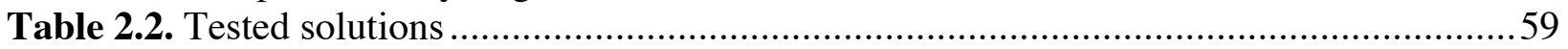

Table 2.3. Pre-filter compositions (* with respect to the weight of HEMA) .........................60

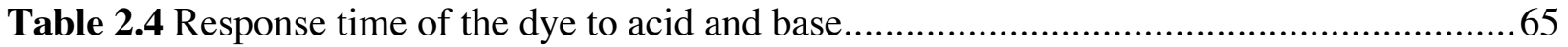

Table 2.5. Comparison between pumping methods for insect cells culture............................83

Table 4.1. Values for swelling (percent change in diameter) and mechanical stiffness (elastic modulus) are given for an array of gel compositions. Both acrylic acid content and crosslinker content are varied. 


\section{Summary}

We present a compilation of the bioflips program including materials development, component development, material characterization, materials modeling, and final system integration. The materials developed include non responsive materials for channel and structural support as well as responsive materials that include both biologically and non-biologically responsive hydrogels. The components developed using the techniques of photopolymerization include valves, filters, mixers, pumps, and sensors. Other components (valves, filters, pumps, sensors) were also incorporated into the microfluidic tectonics platform although made via alternative materials. Materials characterization included testing the hydrogel material for mechanical properties. These properties were then used in the modeling of the swelling and deswelling of hydrogels. The modeling of the hydrogels accurately predicted the physical deformation of the hydrogels. The project ended in the final system integration of the components developed into a bioassay capable of detecting toxin via a visible color change. 


\section{Chapter 1 Materials}

Materials development was key to the success of this project. Having the right materials to use in the fabrication of all the components of the microfluidic tectonics projects was very challenging. However, many materials were developed that consisted of non-responsive materials used to form walls and supports, non-biological responsive materials that changed size in response to physical or chemical stimuli, and biologically responsive materials that responded to specific biologicals.

\section{Non-responsive Materials}

Non responsive materials are those materials used in the microfluidic tectonics process that have no response to the environment but serves as non responsive but functional (walls, channels, supports) to other components of device fabrication.

\section{Construction Material}

A non-responsive material was developed [1] that consists of a monomer, iso-bornyl acrylate (IBA); a crosslinker, tetraethylene glycol dimethacrylate (TEGDMA); and a photoinitiator, 2,2'dimethoxy-2-phenyl acetophenone, (DMPA). This material, referred to as construction material, is photo-definable as shown in Figure 1.1. Construction material is the primary material used to create all the walls, structures, and supports in microfluidic tectonics. It demonstrates a low shrinkage (less than 10 percent) and well-defined characteristics that make it versatile in channel fabrication (see Figure 1.2). Although other monomers were also explored (bis-GMA), the channels used throughout this project consisted primary of the IBA based polymer due to its material properties.

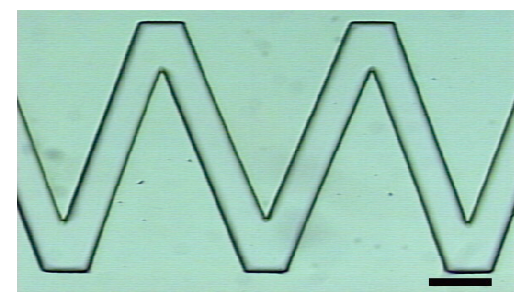

Figure 1.1. Microchannels created using IBA based "construction" material. Bars $=500 \mu \mathrm{m}$.
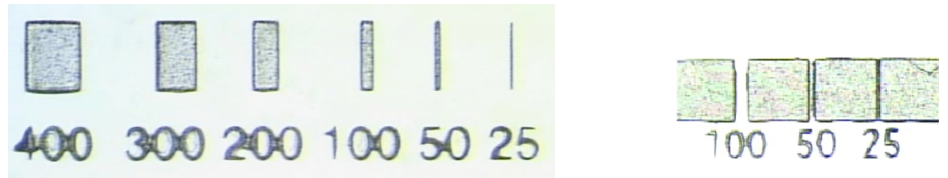

Figure 1.2. Structures showing resolution obtained by using construction material with features down to 25 microns and channel spacing of 100 microns. 
Adhesion of Construction Material

The process of in situ channel fabrication involves a liquid-phase mixture inside a glass channel. However, the inherent volume shrinkage during polymerization causes delamination between the construction material and the glass, leading to leakage. Such delamination occurs consistently, usually several days after the channel fabrication, if not immediately after photopolymerization. A variety of methods were attempted to prevent leakage, such as adding adhesion promoter to the construction material, adjusting the components of the monomer mixture, and surface treatment of the glass channel. It was found that after treating the surface of the glass channel with (3-acryloxypropyl) trichlorosilane (APTCS) (commercially available from Gelest) prior to polymerization, microchannels obtained with $\mu \mathrm{FT}$ could remain leakage-free for a prolonged period of time. APTCS acts as a coupling agent to form covalent bonds between the construction material and the glass (Figure 1.3), therefore improving the adhesion. Examples of such leakage-free microchannels are shown in Figure 1.4.

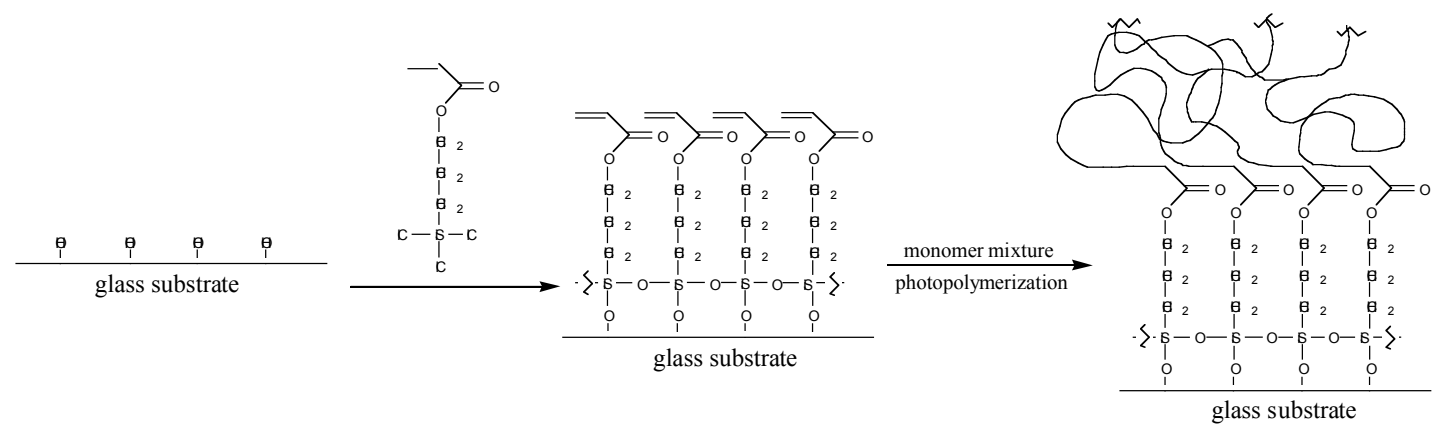

Figure 1.3. A schematic of the surface treatment inside universal glass channels. The silane coupling agent creates covalent bonds between the glass substrate and the construction material during photopolymerizaton

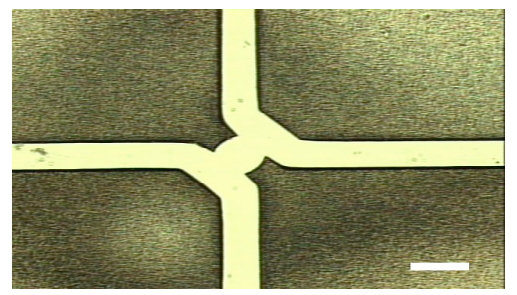

Figure 1.4. Microchannels created with $\mu F T$ that remained leakage-free. Bars $=500 \mu m$.

Although pretreatment solved the problem of adhesion to glass, there may be situations where the shrinkage in the devices could be used to our advantage. A constriction region could be fabricated from the shrinkage property to stop particle movement and allow flow. This method would allow for any device to have a constriction region fabricated inside the channel. Since the 
problem of adhesion applies to rigid glass cavities, a new cavity with other materials or compliant surfaces will also eliminate the shrinkage problem.

\section{Responsive Materials}

Responsive materials are those materials that respond to chemical or physical changes. These materials include hydrogels that respond to changes in $\mathrm{pH}$, chemicals, or temperatures as well as hydrogels polymerized with biological agents that serve as reaction sites. Responsive hydrogel gradients and multifunctional hydrogels are also shown.

\section{PH Sensitive Hydrogels}

The first types of responsive hydrogels that were studied [2] include $\mathrm{pH}$ responsive materials consisting of two monomers, Hydroxyethyl methacrylate (HEMA) and acrylic acid (AA); a crosslinker, ethylene glycol dimethacrylate (EgDMA); and a photoinitiator, 2,2'-dimethoxy-2phenyl acetophenone, (DMPA). These materials were used to show sensing and regulating (from changes in $\mathrm{pH}$ ) inside channels (Figure 1.5) [3].

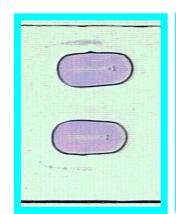

(a)

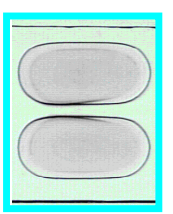

(b)

Figure 1.5. Two pH responsive hydrogel posts are shown inside a channel both before (a) and after (b) being exposed to higher $\mathrm{pH}$.

\section{Dissolvable Hydrogels}

Hydrogels with breakable crosslinkers were developed [4] that responded to a chemical stimuli by dissolving. Figure 1.6a shows the use of dissolvable hydrogels photopatterned into a grid of dissolving and non-dissolving posts. The smaller diameter posts (already seen dissolving by flow of DTT) where made of dissolving hydrogels while the larger posts were made from hydrogel materials with non-breakable crosslinker. In the presence of the de-crosslinking reagent DTT, the smaller hydrogel posts dissolved completely (Figure 1.6b) while the other hydrogel posts were not dissolved.

\section{Temperature Sensitive Hydrogels}

A temperature sensitive hydrogel was investigated as a precursor to the DNA responsive hydrogels. A temperature sensitive hydrogel is first photopolymerized and then chemically modified to produce an amine terminated polymer. Solid-phase coupling of the PNA monomers to the hydrogel may then be conducted. Temperature sensitive polymers can be achieved by neat copolymerization of the monomers butoxymethylacrylamide (BMAAm) and $N, N-$ 
dimethylacrylamide (DMAAm). Addition of acryloylsarcosine methyl ester (ASME) to the mixture provides a temperature sensitive polymer that can be chemically modified for eventual in-channel solid phase peptide/PNA synthesis (Figure 1.7). Increasing the BMAAm content lowers the transition temperature of the polymers, and polymerization in $\mathrm{H}_{2} \mathrm{O} / \mathrm{EtOH}$ results in an increase in \% change in area. Successful incorporation of the $\mathrm{pH}$ sensitive monomer, acrylic acid (AA), was conducted to study the swelling behavior induced by both $\mathrm{pH}$ and temperature. Small amounts of AA can be capable of inducing a large change in volume by the $\mathrm{pH}$ induced swelling of AA substituted gels. These swollen gels were also induced to contract at higher temperatures. This approach produces a temperature sensitive polymer onto which amino acid or PNA monomers can be covalently coupled.

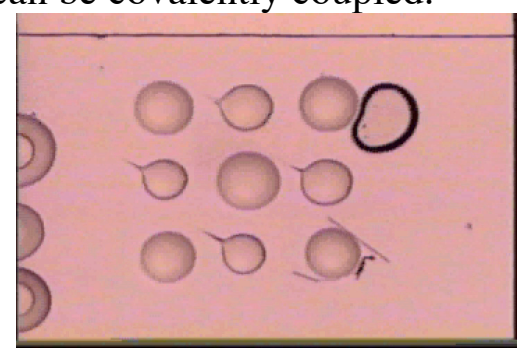

(a)

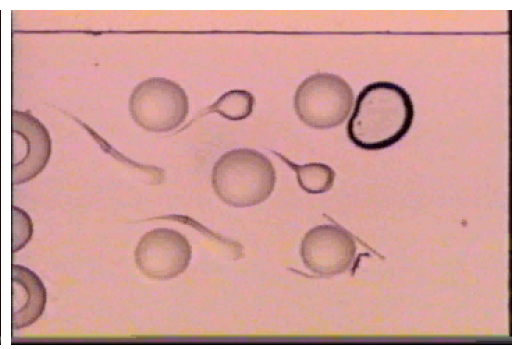

(b)

Figure 1.6. A photopolymerizable hydrogel with disulfide crosslinks dissolves in the present of DTT.
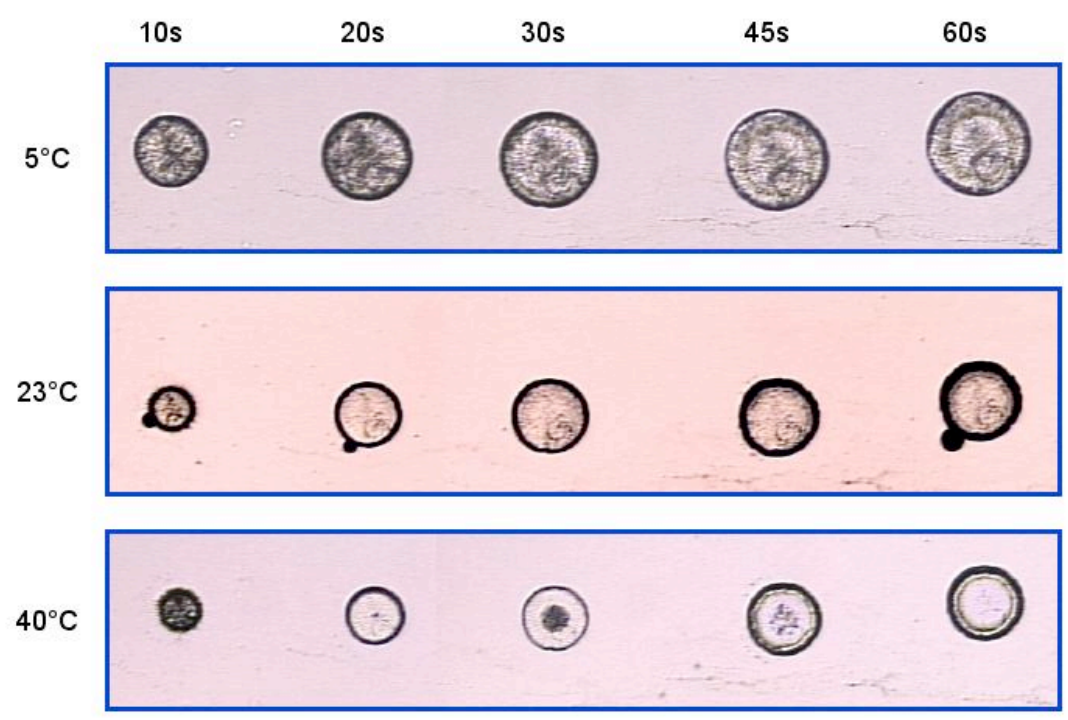

Figure 1.7. Temperature sensitivity of BMAAm/DMAAm/ASME gels at different polymerization times (10 - 60 seconds) for different temperatures $\left(5^{\circ} \mathrm{C}, 23^{\circ} \mathrm{C}\right.$, and $\left.40^{\circ} \mathrm{C}\right)$ Gels were photopolymerized through a 400 um diameter circle. 


\section{Biosensing Hydrogels}

In the early stages of the project, hydrogels that were made from acrylamide were use to trap biomolecules. Because these gels do not responds to a biological but rather have a biological agent trapped inside, they were classified as biosensing hydrogels. In order to create methods that would allow us to test the other components of the autonomous system (valves, filters, pumps) in the interim, this simple and less sophisticated technique was utilized. The end goal is to chemically engineer hydrogels that can act as both the detection (agent of interest attaches to gel) and sensing (gel changes color from secondary reaction).

In order to create a simple scheme, the readout, which utilized alkaline phosphatase, was combined with the enzyme Horseradish peroxidase (HRP). The enzyme is used quite frequently in signal amplification for Enzyme Linked Immunosorbent Assays, ELISAs, and its use increases the range of readouts available for the assay. An antibody with an enzyme conjugate (HRP) was incorporated into the acrylamide gel mixture and polymerized. Two substrates were used for evaluation of the assay: 4-chloro-1-napthol $(4 \mathrm{C} 1 \mathrm{~N})$, which produces a blue color (Figure 1.8a) and 3-amino-9-ethylcarbazole (AEC) which produces a red color (Figure 1.8b). These results offer examples of an additional enzymatic process that can be performed within a gel to produce a colorimetric readout.

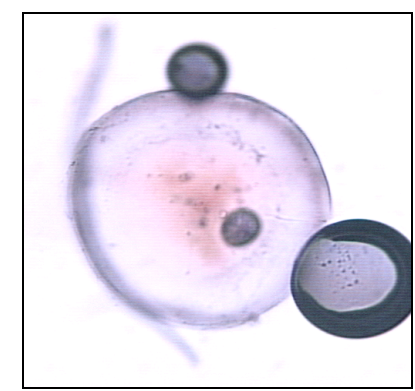

(a)

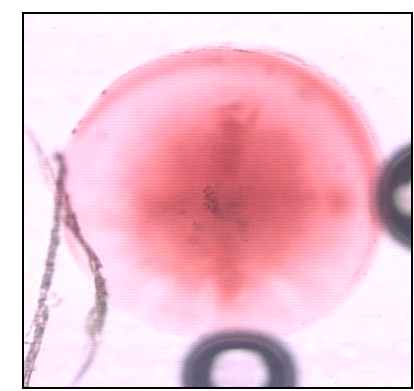

(b)

Figure 1.8. A hydrogel post containing antibody with HRP conjugate after exposure to 4chloro-1-napthol (4C1N) shows a blue color (a) and after exposure to 3-amino-9-ethylcarbzole (AEC) shows a red color (b). Post sizes are $300 \mu \mathrm{m}$.

Combining the previous concepts, a sandwich ELISA protocol was developed which incorporates directed flow of reagents in microchannels. Inactivated botulinum toxoid (type B) as well as Rabbit IgG Anti-Botulinum type B (biotinylated) was provided by the Johnson lab. Current microtiter plate protocol calls for coating the surface with $1 \mu \mathrm{g} / \mathrm{ml} 1^{0}$ antibody and, with this concentration, detection levels as low as 3-5 pg can be achieved. The initial antibody concentration will be much higher in the hydrogel protocol being tested until the experimental conditions are optimized, but initial results are very encouraging. Visible detection of the toxin has been achieved (Figure 1.9), and the concentrations should be much lower after optimization is completed. The protocol for the sandwich ELISA is shown in Figure 1.9. 


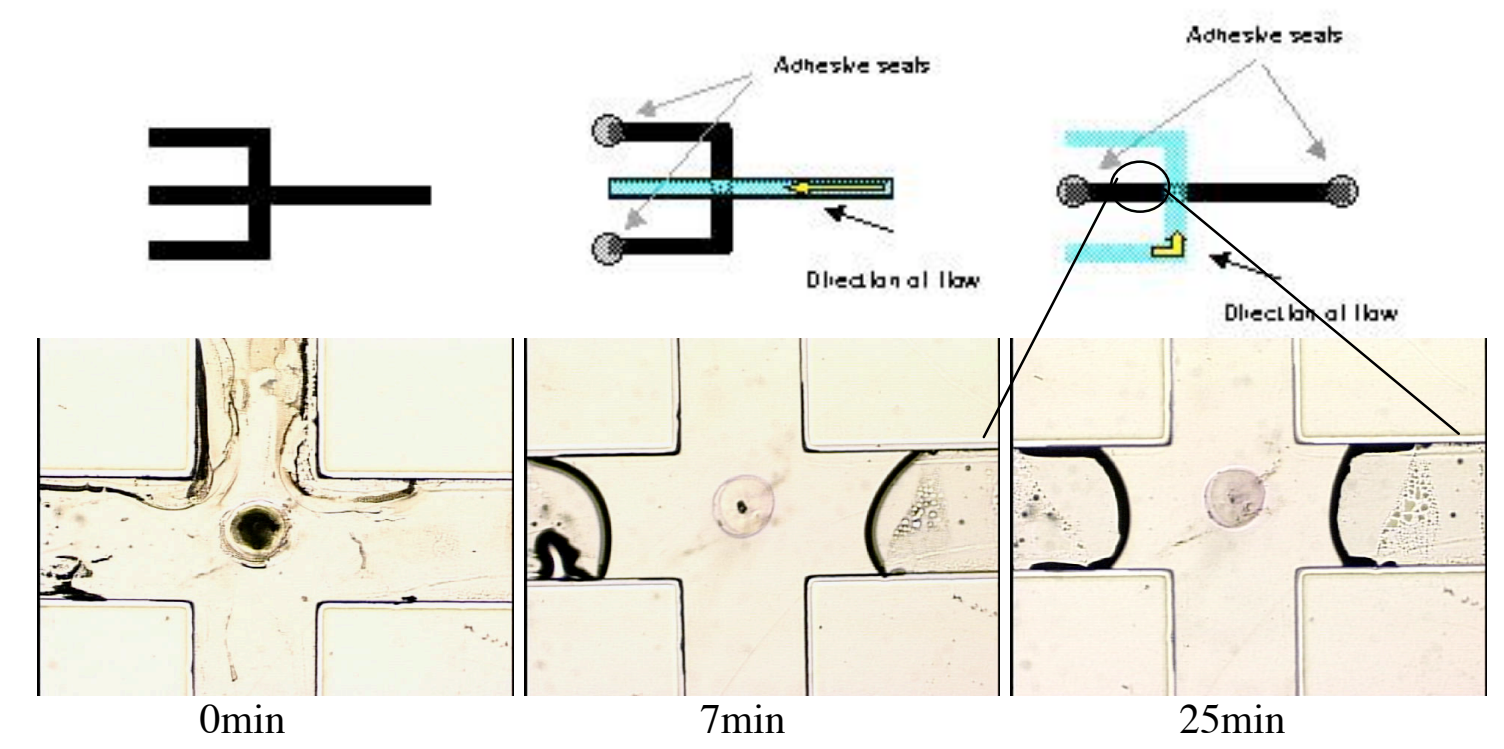

Figure 1.9. Color development of sandwich ELISA in hydrogel after the addition of substrate over time.

These initial studies demonstrated that biological materials could be contained within the hydrogels, dried, stored, and still retain functionality. This hydrogel material was then optimized to produce a readout structure, which could be seen directly by the human eye without the need of any instrumentation or electronics. For more information on biosensors, please see the components section.

\section{Surface Studies of Hydrogels}

Immobilization of fluorescein-labeled bovine serum albumin (FITC-BSA) protein to hydrogel surfaces was investigated. To demonstrate the utility and versatility of a hydrogel system capable of biomolecule immobilization, we have employed reductive amination to conjugate proteins displaying lysine residues to aldehydes localized on the perimeter of a patterned hydrogel (Figure 1.10). FITC-BSA was conjugated to the surfaces of 3:1 glycerol monomethacrylate-co-acrylic acid (GMM-co-AA) hydrogels that were oxidized with $\mathrm{NaIO}_{4}$ for $10 \mathrm{~min}$ to present aldehyde groups on the outer $30 \mu \mathrm{m}$ of the hydrogel. The protein immobilization process used a $\mathrm{pH} 7.4$ phosphate buffer containing $1.5 \mathrm{mg} \cdot \mathrm{ml}^{-1}$ FITC-BSA and $10 \mathrm{mg} \cdot \mathrm{ml}^{-1} \mathrm{NaCNBH}_{3}$ that was introduced into the microchannel. These conjugation conditions were beneficial in speeding the diffusion of the protein into the polymer network owing to the swelling of the hydrogel under the basic conditions. After washing with phosphate buffer to remove non-conjugated FITC-BSA, confocal microscopy was used to evaluate the location of the protein. The oxidized hydrogels conjugated with FITC-BSA showed fluorescence (Figure $1.10 \mathrm{~b})$ near the surface similar to previous hydrogels labeled with the small fluorescent molecule, lucifer yellow. Control hydrogels, GMM-co-AA hydrogels not oxidized with $\mathrm{NaIO}_{4}$ 
but subjected to the reductive amination with FITC-BSA, showed little fluorescence under the same visualization conditions (Figure 1.10c). These results suggest that the aldehyde containing hydrogels are applicable to many of the current biochemical techniques employing gluteraldehyde as a linker molecule. These applications include the immobilization of proteins for bioreactors or biosensors onto a unique, $\mathrm{pH}$-responsive hydrogel scaffold.

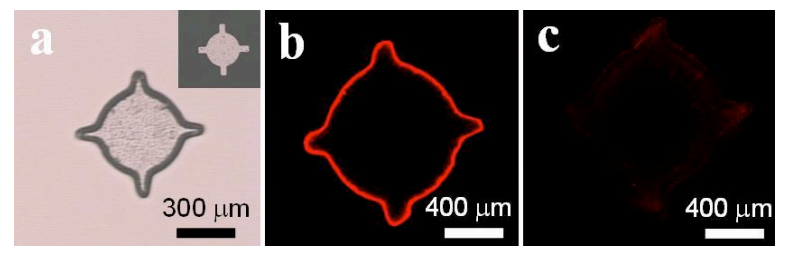

Figure 1.10. An optical micrograph of a GMM-co-AA hydrogel with a spike-circle design (a). Reductive amination of FITC-BSA to a $\mathrm{NaIO}_{4}$ oxidized hydrogel (b) and a non-oxidized hydrogel (c) was attempted using $\mathrm{NaCNBH}_{3}$. Only the oxidized hydrogel retained the fluorescent protein after washing.

\section{Dual Response Hydrogel Posts}

Hydrogels that swell in response to acidic or basic conditions (i.e., pH-responsive hydrogels) have been extensively studied due to their ease of induced swelling (all one needs is a change of buffer solution) and their relevance to $\mathrm{pH}$ changes that occur in biological systems. Previous work on $\mathrm{pH}$-responsive hydrogels utilized only a single ionizable monomer within the polymer matrix. Here, we have prepared hydrogels composed of two ionizable monomers that have opposing acid-base responses. Employing a pre-polymer mixture containing a hydrophilic monomer 2-hydroxyethyl methacrylate (HEMA), ionizable monomers methacrylic acid (MAA) and 2-dimethylaminoethyl methacrylate (DMAEMA), and a photoinitiator 2,2'-dimethoxy-2phenyl acetophenone, hydrogels were prepared through an in situ photo-polymerization within a microchannel. This polymerization scheme not only allows the rapid preparation of hydrogel structures, but also allows the incorporation of varying concentrations of opposing acid-base functionality in a single step through the free radical polymerization of the methacrylate functional groups.

Three hydrogels were prepared with 80 mol\% HEMA, and $20 \mathrm{~mol} \%$ of combined MAA and DMAEMA with ratios of $1: 1,2: 1$, and 1:2 (i.e., 10:10, 13.6:6.3, 6.3:13.6 mol\%). When subjected to a range of $\mathrm{pH}$ buffers, the equilibrium swelling of these hydrogels was dramatically different. As shown in Figure 1.11, all three hydrogels displayed a response to both high and low $\mathrm{pH}$; however, each composition showed a different $\mathrm{pH}$ volume transition. The dual response of these hydrogels is easily understood, as at low $\mathrm{pH}$ the amine groups are protonated while at high $\mathrm{pH}$ the carboxylic acids are deprotonated. Both of these effects would create an osmotic pressure difference between the inside and outside of the hydrogel and the amount of swelling is directly dependent on the amount of each ionizable monomer added. Hydrogels with 1:1 MAA:DMAEMA swelled to a similar extent in both acidic and basic conditions. However, 
hydrogels with more of one monomer (i.e., 2:1 MAA:DMAEMA) swelled greater in the $\mathrm{pH}$ that elicits the more concentrated monomer to swell. These hydrogels may have utility in applications where a hydrogel valve or actuator is needed to respond to acidic or basic conditions (i.e., swells when the specific $\mathrm{pH}$ is not maintained).

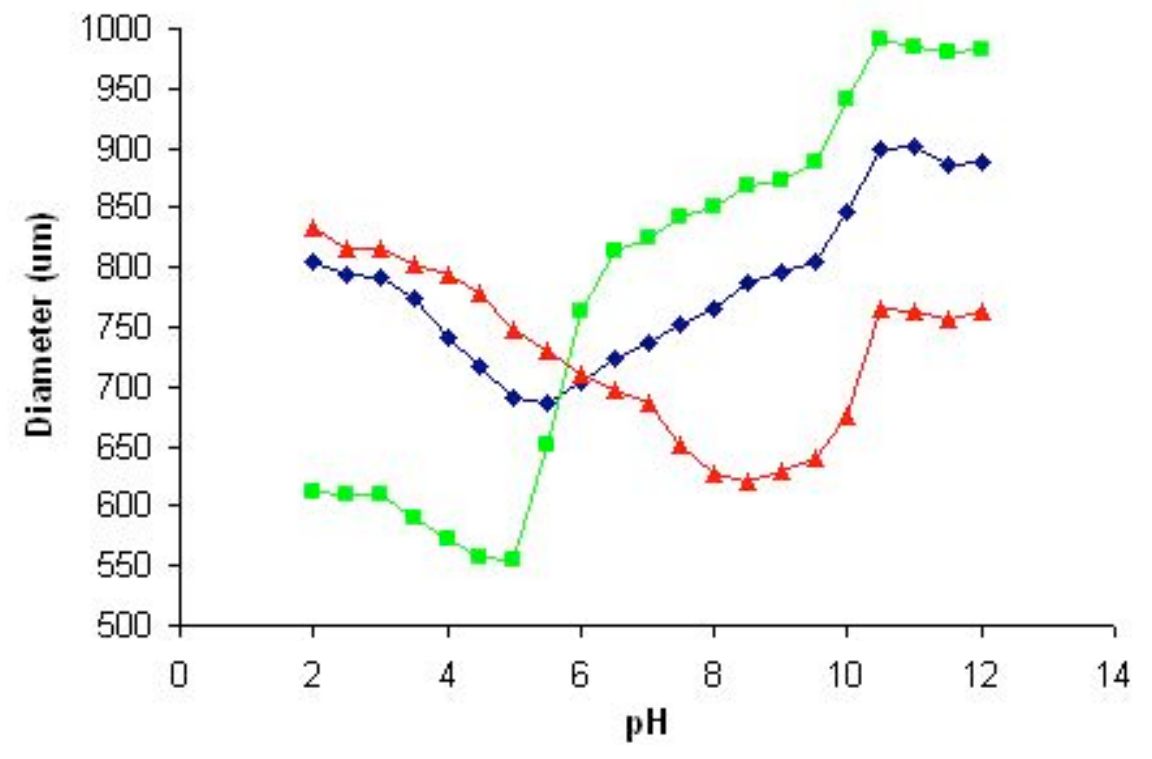

Figure 1.11. Swelling of hydrogels with 80\% HEMA and 20\% ionizable monomers MAA and DMAEMA in different ratios [1:1 $\diamond), 2: 1(\square)$, and 1:2 $(\pi)$ ]. Hydrogels were bathed in buffers from $\mathrm{pH} 2$ to $\mathrm{pH} 12$.

Dual $\mathrm{pH}$-responsive core-shell hydrogels were also made containing both a vinyl pyridine (VP) component and a 2-dimethylaminoethyl methacrylate (DMAEMA) component were prepared using an in situ photopolymerization process. Complementary photomasks were utilized to prepare hydrogels with core:shell volume ratios of 2:1, 1:1, and 1:2. Depending on the location of each polymer component, dramatically different swelling profiles were achieved. Selective swelling of the shell followed by the core components allowed the hydrogel to expand with the usual kinetics; however, by switching the location of each polymer component and swelling the core first, swelling rates decreased by over an order of magnitude and were dependent on the shell component's volume (Figure 1.12). The ability to patterned core:shell volumes also provided the ability to fabricate hydrogels that possess a constant maximum diameter but different cut off points between its first and second transition volumes. These materials may be of interest for controlled release applications.

\section{Hydrogel Gradients}

Microfluidic environments allow for precise and unique control of the local fluid environment. One of the consequences of laminar flow (typical in microchannels) is that the mixing between two or more streams flowing in contact is diffusion dependent. Such diffusion dependency is 
utilized in generating gradients where a range of concentration of protein or analytes as external stimuli could be applied to biological systems. Similarly, flow gradients could be further manipulated to generate scaffolding material with inherent chemical/biochemical gradients for studying the effects of surface properties on cell/tissue development/mobility.

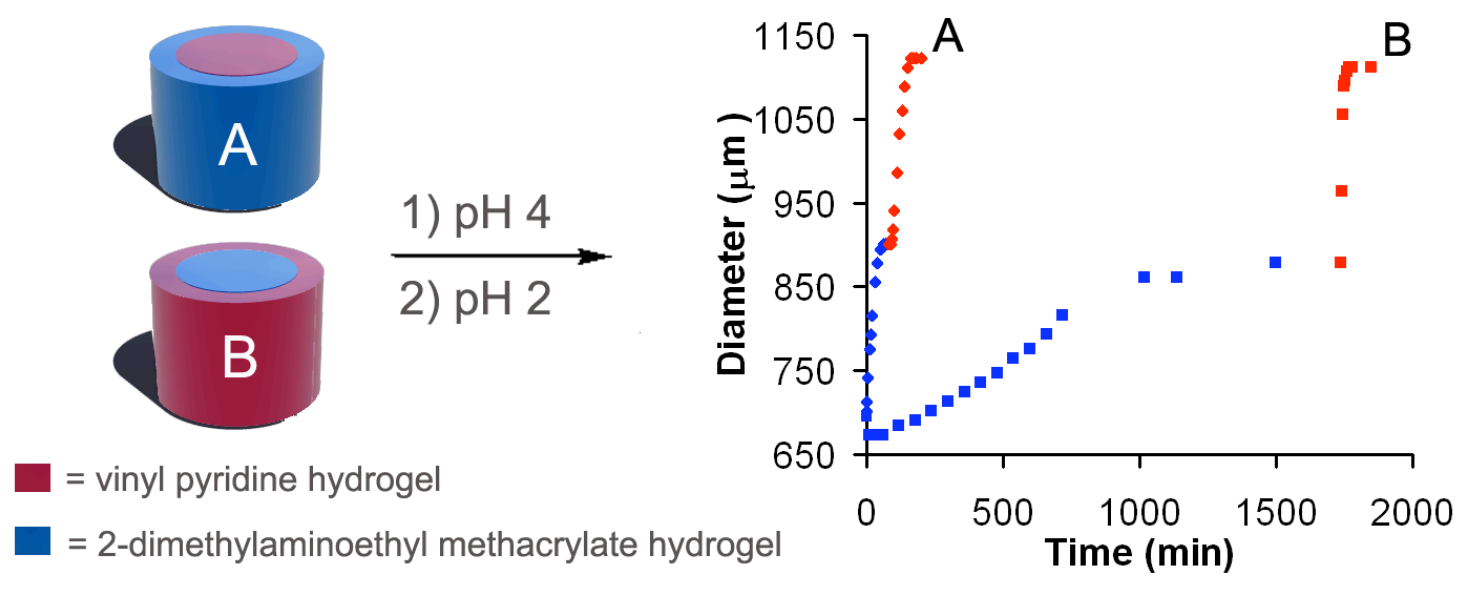

Figure 1.12. Dual responsive hydrogel posts are shown with different core shell ratios of swellable hydrogels.

To demonstrate the gradient idea, we have created asymmetric responsive materials using gradient surface polymerization that will be the basis of generating scaffolding or smart material.

Asymmetric microstructures are made by utilizing laminar flow and molecular exchange at the boundary layer between the two parallel flows down the channel (Figure 1.13). The parallel flows contained two different concentrations of crosslinker. The asymmetry can be controlled by varying the initial crosslinker concentration and the extent of diffusion between the two flows. Furthermore, the gradient generation can be applied to many different chemical systems where the asymmetry could be triggered by varying stimuli.

The gradient surface polymerization uses an additional flow stream on top of the two prepolymer mixtures used for creating the molecular gradient. A simple cross sectional view of the flow streams are shown in Figure 1.14.

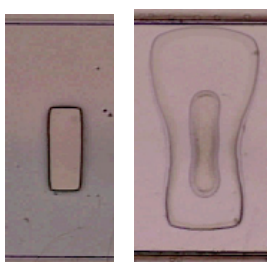

Figure 1.13. A hydrogel structure made inside a microchannel with a gradient of crosslinker is shown in the contracted (left), expanded (right) state. 


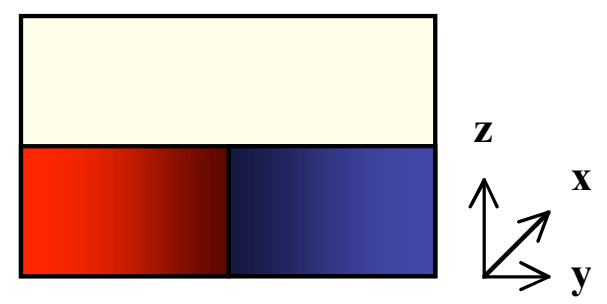

Figure 1.14. A schematic view of the cross section of the channel is shown above. The two bottom flows generate the molecular gradient and the third top flow functions as the "spacer" allowing for polymerization of the bottom surface only.

Figure 1.15 shows the gradient surface polymerization of the bottom layer of the channel. Here the diffusion in the boundary layer of the two bottom flows is tracked by $20 \mathrm{~nm}$ fluorescent microspheres that were present in one of the bottom streams. The bright image indicates that the polymerization has occurred only at the bottom of the channel.
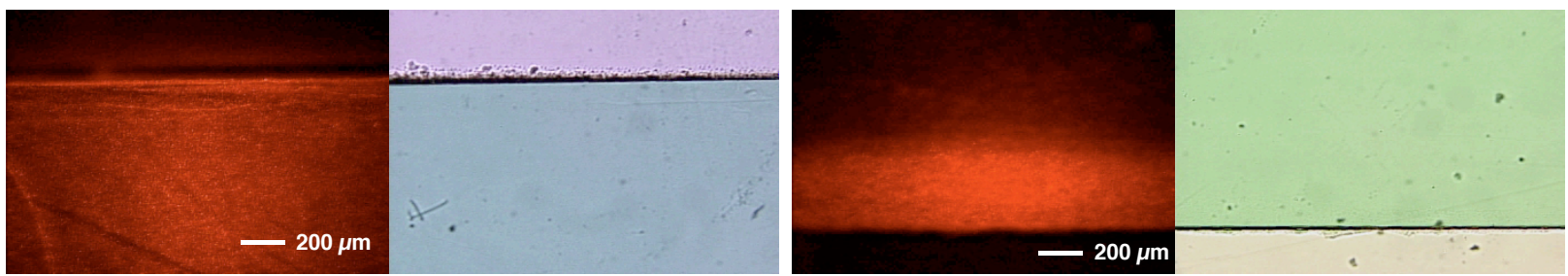

Figure 1.15. Surface polymerization of an acrylamide gel (fluorescent beads) and corresponding white image with blue water indicates surface layer polymerization (left). A gradient surface polymerization of acrylamide gel (fluorescent beads in lower stream) and corresponding white image with green water are shown (right).

The generation of dual response materials with asymmetric microstructures and surface gradient polymerization is an important addition to the microfluidic toolbox that can be utilized to control microenvironment parameters.

\section{Biologically Responsive Materials}

Biologically responsive materials involve methods of invoking a response from a material as well as direct stimuli responsive hydrogels to biologicals. These methods include lipid layers which can surround a hydrogel giving it a cell-like functionality. Liposomes also respond by having sensitive surfaces that lyse to produce secondary responses. Membranes are also developed that are not only functional as barriers but are also infused with biospecific degradable properties which can be used as sensors. 


\section{Lipids}

One approach to bioresponsive materials is the creation of a biologically responsive membrane around a hydrogel structure [5]. Stimuli-responsive hydrogels have been modified with a fatty acid layer to create a cell-like object, which will be referred to as a "cell-gel". Since the lipid layer is selectively permeable, a different $\mathrm{pH}$ can be maintained in the hydrogel, allowing it to remain contracted while bathed in a $\mathrm{pH}$ solution that would normally cause expansion. Upon the exposure of the surface receptor to a membrane-disrupting agent, the lipid layer will become permeable to ions causing the hydrogel to swell and consequently amplify the signal. The lipid coating performs the detection, and the hydrogel matrix amplifies the detection into a signal that is visibly observable to the operator without the need for a separate energy source. The approach for creating the lipid-modified hydrogel is illustrated in Figure 1.16.

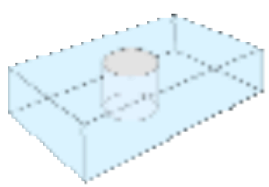

a)

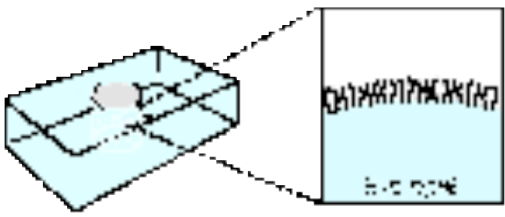

b)

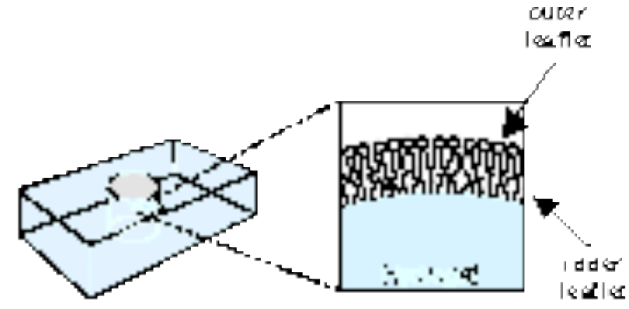

c)

Figure 1.16. Cell-gel fabrication shows a microgel cylinder $(a)$ is polymerized in a microchanel. Fatty acid chains $(b)$ are covalently linked to the surface to create the inner leaflet, promoted by the amphiphilic nature of the interface. Phospholipids are self-assembled around the exterior. This object maintains a pH gradient across the lipid coating for several days.

To test the efficiency of the lipid layer to form an ion barrier, the modified microgel ( $\mu$ gel) was exposed to a basic solution (which would normally cause the hydrogel to swell), and the $\mu$ gel diameter was measured at timed increments. It was found that hydrogels modified in this manner remain stable for a several days without visible change in color or size, demonstrating the ability to maintain chemical gradients. In contrast, unmodified hydrogels that contain a $\mathrm{pH}$ indicator (phenolphthalein) become fully expanded within 40 minutes accompanied by a color change from colorless to pink. The way in which the $\mu$ gel is modified is not clear, but by puncturing the lipid layer, a local swelling in the $\mu$ gel was observed (Figure 1.17). This showed that ion influx caused the immediate region to swell, and eventually lead to expansion and color change in the entire $\mu$ gel and that the lipid layer did not functionally change the responsiveness of the $\mu$ gel.

The lipid layers surrounding the hydrogel material should behave like a cell and have cell like characteristics (cell-gel). The disruption of lipid bilayers by surfactants has been extensively characterized in the literature and should affect the cell-gel much the same way. This process involves the interchain penetration of surfactants into the lipid layer, thereby increasing the physical properties, such as permeability of the lipid layer and ultimately resulting in its 
solubilization. However, it remains to be determined if surfactants can increase the permeability of a lipid layer composed of fatty acid chains covalently anchored to a surface, as is the case with the "cell-gel" structure we created. The effects of two surfactants, sodium dodecyl sulfate (SDS) and Triton X-100, on $\mu$ gels with fatty acids covalently linked to the surface were examined by taking modified $\mu$ gels and exposing them to a solution of surfactant dissolved in $\mathrm{pH} 12$ buffer. The $\mu$ gels were modified by flowing a $0.1 \mathrm{M}$ palmitoyl chloride, $0.1 \mathrm{M}$ triethyl amine, and 0.01 M 4-(dimethylamino) pyridine in benzene through the channel at a rate of $5.0 \mathrm{~mL}$ per hour for 8 hours.

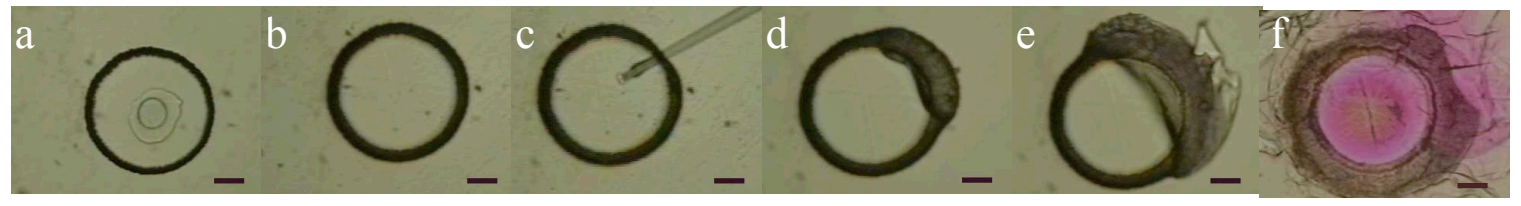

Figure 1.17. A pH sensitive hydrogel modified by covalently linking palmitolyl chloride to the surface. When a $\mathrm{pH}=12$ buffer was flowed into the channel (a), the modified $\mu$ gel remained stable for hours without obvious signs of change while the unmodified $\mu$ gel of the same size and composition started to swell instantly $(b)$. After physically disrupting the fatty acid layer by piercing the $\mu \mathrm{gel}$ with a micropipette tip (c), the modified $\mu \mathrm{gel}$ began to expand at the disrupted location (d,e) and eventually became fully swollen accompanied by the phenolphthalein indicator's color change (f). Scale bars are $100 \mu \mathrm{m}$.

Prior to the exposure of the modified $\mu$ gels to the surfactant, all of the $\mu$ gels were placed in a $\mathrm{pH}$ 12 buffer solution to demonstrate that the fatty acid layer attached to the surface created an ion barrier. The ions in the external buffer solution were unable to enter into the $\mu$ gels and consequently trigger $\mu$ gel expansion. However, the addition of the surfactant to the buffer solution increased the permeability of the fatty acid layer to the ions in the buffer solution.

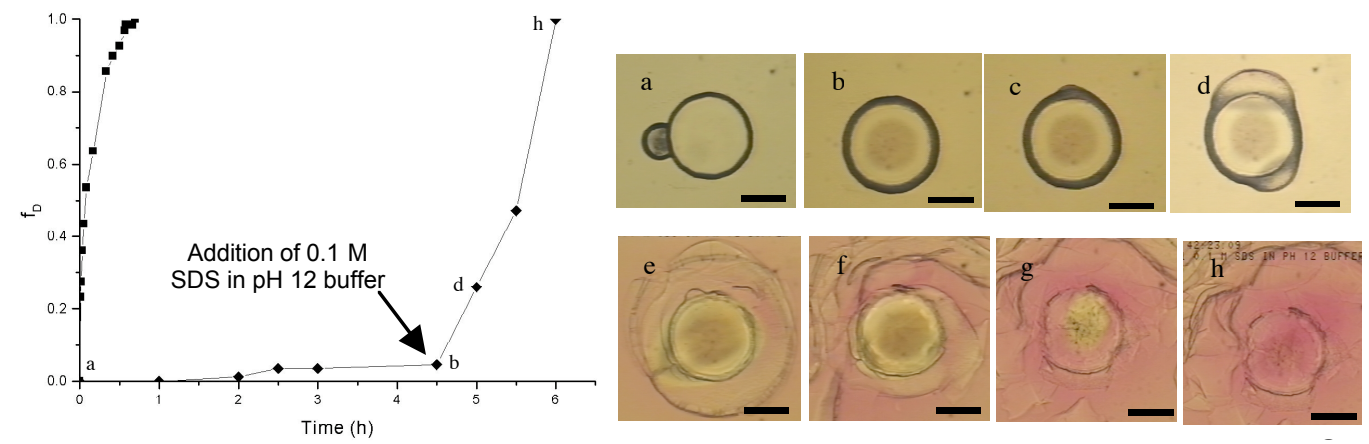

Figure 1.18. In contrast to an unmodified $\mu \mathrm{gel}(\boldsymbol{\square})$, a ugel covalently modified (a) with fatty acids ( ) doesn't expand in pH 12 buffer solution (b) until SDS was added to the buffer. The expansion quickly began at points on the $\mu \mathrm{gel}$ surface $(c)$, which propagated $(d-g)$ until the entire $\mu g e l$ had expanded (h). Scale bar is $250 \mu \mathrm{m}$. 
This fact was verified by the abrupt expansion of the $\mu$ gels after the addition of the surfactant (Figure 1.18). A series of concentrations were explored for SDS, as well as for Triton X-100. Ten modified $\mu$ gels were tested at each concentration for each surfactant, and the average time for expansion to the $2 / 3 \mathrm{~F}_{\mathrm{D}}$ (where $\mathrm{F}_{\mathrm{D}}$ is the fractional change in the $\mu$ gel diameter) was determined. This data was also used to determine the $95 \%$ and $99 \%$ confidence interval, and the statistical analysis was used to predict with the noted confidence the average time it will take a $\mu$ gel to swell to the $2 / 3 \mathrm{~F}_{\mathrm{D}}$ for each concentration and surfactant (Figure 1.19). It can be seen that expansion is the fastest when a higher concentration of surfactant is used and is slightly faster for Triton X-100 than SDS at the higher concentrations. This shows that permeability of the covalently attached fatty acid layer was induced by the addition of surfactants much like a real cell. This effect also allows the membrane to remain intact until the introduction of a chemical stimulus that will cause the membrane to rupture.

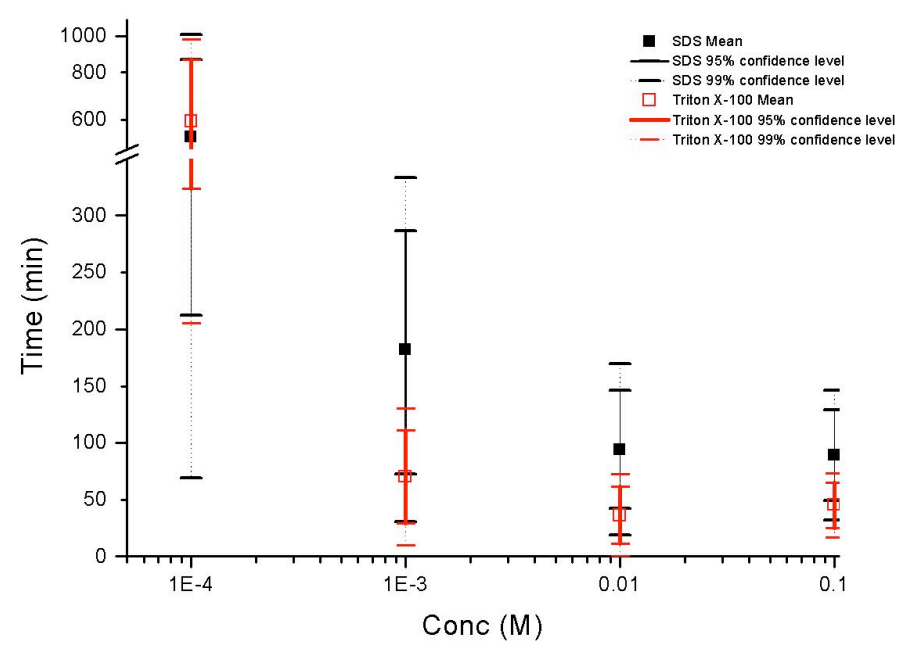

Figure 1.19. Plot of the mean expansion time for $2 / 3 F_{D}$ as a function of the surfactant concentration.

A $2^{\text {nd }}$ leaflet of phospholipids around a fatty acid modified $\mu$ gel was also fabricated. In order to create a cell-like surface on a $\mu \mathrm{gel}$, it is believed that the formation of the second leaflet of the bilayer through the assembly of phospholipids around the $\mu$ gel modified with a layer of fatty acids (Figure 1.20) is an essential step. It is crucial that this layer have a finite thickness and uniform packing to allow cell-like interactions between the modified $\mu$ gel and membrane proteins.

Previous literature reports have demonstrated that a phospholipid monolayer can by assembled on a hydrophobic surface through the introduction of vesicle solutions to the surface ${ }^{1}$. There are a multitude of parameters that must be adjusted in order to modify these procedures to be compatible with this system. These modifications include a method for forming vesicles, the lipid composition, what solvent (purely aqueous, buffered, organic, or a combination of both) the 
vesicles should be in when introduced to the modified $\mu$ gel, the vesicle concentration, the length of the incubation period, and the temperature of incubation.

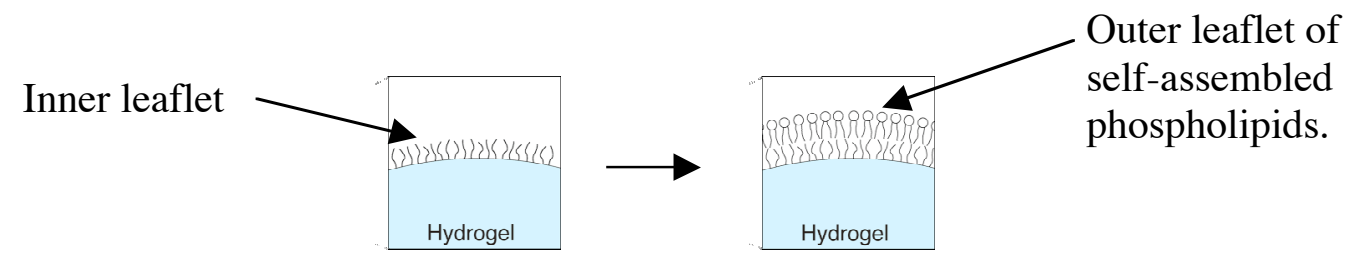

\section{Figure 1.20. Phospholipids are self-assembled around the modified $\mu$ gel to form the second leaflet of the bilayer.}

Experiments were performed with vesicle suspensions made in solutions of various $\mathrm{pH}$ and ionic strength either by sonicating dried lipids in aqueous solutions or through an injection process 1 . The phospholipid composition included 1,2-dioleoyl-sn-glycero-3-phosphocholine (DOPC)/ 1,2dioleoyl-sn-glycero-3-phosphoethanolamine-N-(7-nitrobenaoxadiazol-4-yl) (DOPE-NBD) or DOPC/ cholesterol/ 1,2-dioleoyl-sn-glycero-3-[phospho-rac-(1-glycerol)] [sodium salt] (DOPG)/ triolein/ DOPE-NBD mixtures, where the DOPE-NBD lipid has a fluorescent label at the head group region.

Bilayer formation was initiated by flowing the aqueous vesicles suspensions into channels containing fatty acid- modified $\mu$ gels, allowing the phospholipid layer to assemble for various time increments at various temperatures, then removing the excess phospholipids by flowing water through the channels. Characterization was performed on a newly acquired Leica SP2 confocal microscope. The presence of phospholipids on the $\mu$ gel was determined by the fluorescent signal emitted by the fluorophores attached to the phospholipids. A quenching technique was used to assess the thickness of the phospholipid layer. This technique is similar to one used for characterizing the lamellarity of vesicles. Briefly, $\mathrm{Na}_{2} \mathrm{~S}_{2} \mathrm{O}_{4}$ reduces the fluorescence intensity of the NBD fluorophore through the reduction of the fluorophore's nitro substituient. Since the phospholipid layer is impermeable to ions, only the fluorophores located at the phospholipid/water interface react with the ionic quencher, resulting in an increase in the fluorescence signal in this region. When employed to investigate the thickness of a phospholipid layer assembled on a fatty acid- modified $\mu$ gel, only the fluorophores attached to the most exterior phospholipid layer are quenched. Consequently, if the phospholipid layer on the modified $\mu \mathrm{gel}$ is a monolayer, as desired, the fluorescent intensity of the sample should be undetectable after the application of the quenching solution.

Confocal imaging of phospholipid layers assembled on the fatty acid- modified $\mu$ gels has demonstrated that a fluorescent lipid layer was present on the periphery of the $\mu$ gels (Figure 1.21). The intensity of this layer varied according to the z-position (height location) in the sample. When the quenching solution was applied to the samples, a range of fluorescent intensity decrease was observed, however none of the samples had a complete disappearance of the fluorescent signal. This implies that the phospholipids were assembled into more than one 
layer on the surface, and further investigation is necessary to improve the phospholipid layer assembly procedure.
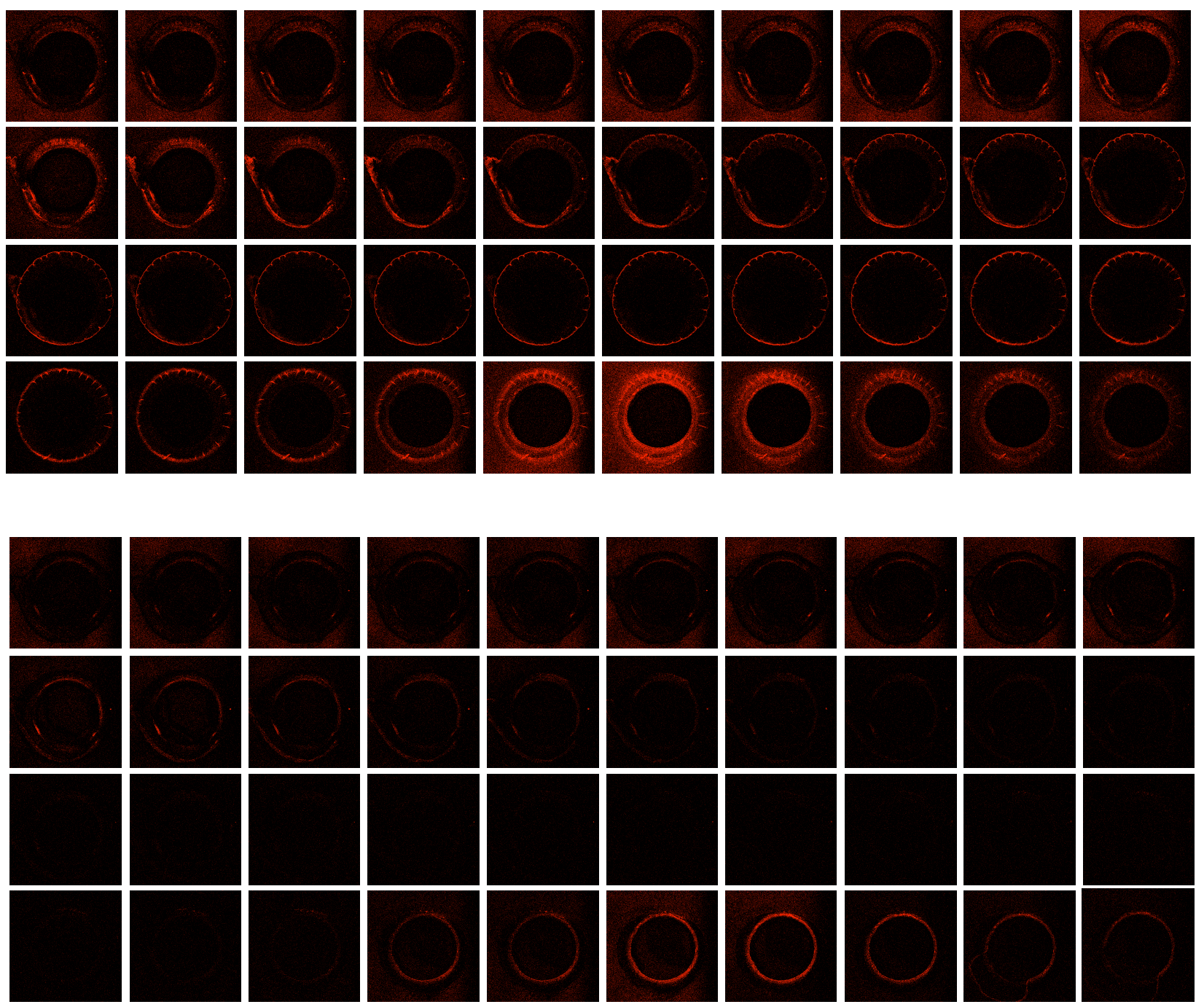

Figure 1.21. The upper block of images corresponds to confocal images of a phospholipid layer assembled on a fatty acid modified $\mu$ gel while in water. The lower block of images is the same sample imaged under the same parameters immediately after a $10 \mathrm{mM} \mathrm{Na}_{\mathrm{s2}} \mathrm{S}_{2} \mathrm{O}_{4}(\mathrm{Ph} \mathrm{11})$ quenching solution was introduced to the channel. The decrease in fluorescence intensity in the lower block of images is due to the interaction of the ionic quencher with the fluorophores attached to the head groups of phospholipids at the lipid/ water interface.

Experiments were conducted to determine if the 2-step bilayer formation approach had biological response potential to channel forming membrane proteins. Specifically, the response to the channel protein melittin, a bee venom component, was examined since the addition of this 
protein to vesicle solutions eliminates a $\mathrm{pH}$-gradient, as previously demonstrated in numerous publications and confirmed in our laboratory. However, the addition of melittin did not trigger $\mu$ gel expansion as expected, which may indicate the protein didn't span the membrane or incorporate into the lipid layer. Inability of the channel to span the lipid layer might be because the phospholipid layer is too thick, and the role that hydrogel surface reorganization plays in this process still needs to be explored. These complications have lead to the amendment of the current approach, as schematically illustrated in Figure 1.22

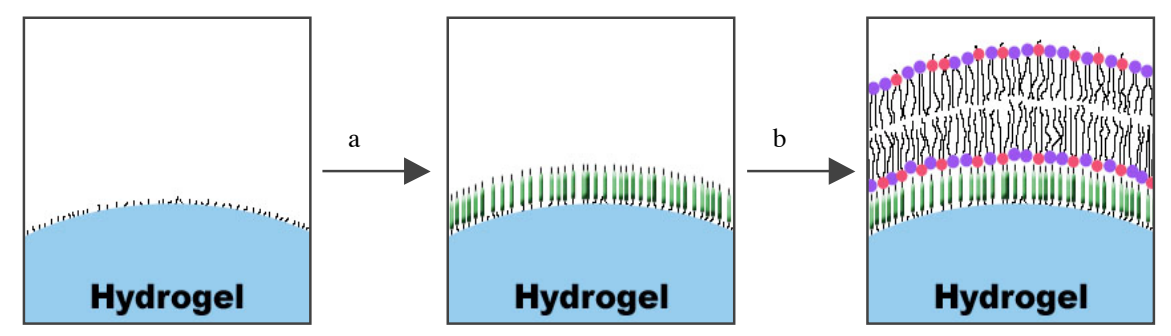

Figure 1.22. A hydrophilic spacer (a) is attached to the surface of the ugel to allow for the incorporation of transmembrane proteins. Then vesicles formed with ITC-lipids $(b)$ are fused to the $\mu \mathrm{gel}$. Reaction of the ITC moiety with the amines presented by the tether would result in the formation of a covalent urethane linkage.

Since the covalent attachment of lipids directly to the substrate has been reported to negatively influence transmembrane protein incorporation and function, a hydrophilic spacer, such as a glycine-glycine peptide, will be attached to the surface of the hydrogel. Once attached, a phospholipid bilayer will be self-assembled on the surface of the $\mu$ gel with a single step process, as opposed to the two separate modification procedures previously employed. Bilayers would be formed by the fusion of vesicles containing the reactive phospholipids to an unmodified $\mu$ gel. The reactive phospholipid would have an ethanolamine headgroup modified with an isothiocyanate moiety. Such phospholipids can be synthesized with a one step process and selectively react with amino functionalities in the presence of water. Incorporation of reactive lipids into vesicles and bilayer formation through their fusion onto a $\mu$ gel surface is predicted to form a phospholipid layer in a single step (Figure 1.23).

Saturated $n$-alkyl fatty acid chlorides $\left(\mathrm{CH}_{3}\left(\mathrm{CH}_{2}\right)_{n} \mathrm{COCl}\right.$ where $n=0,2,4,6,8,10,12$, and 14) were covalently attached to $\mathrm{pH}$-sensitive hydrogel objects ( $\mu$ gels) using an in situ process (Figure 1.24). Staining with a lipophilic dye indicated that the resulting fatty acid layer was confined to the periphery of the $\mu$ gel. The barrier properties of these fatty acid coatings were investigated by determining the half-life of $\mu$ gel expansion after exposure to a buffer solution that triggers swelling of unmodified $\mu$ gels. For $n=0$, the half-life values were similar to unmodified $\mu$ gels, indicating that no significant ion gradient was established. 


\section{Surfaces of Lipid Modified Gels}

The design, synthesis, and characterization of stimuli-sensitive hydrogels, which are capable of chemoselective ligation of lipids and other biomolecules (ie. proteins) in microchannels was investigated. A developed strategy for lipid modification involves the preparation of hydrogels containing aldehydes at the hydrogel surface, which can be used as a conjugation site. Glycerol monomethacrylate-co-Acrylic acid was polymerized to form $\mathrm{pH}$-sensitive hydrogels with pendant 1,2-diol groups. The 1,2-diol can then be mildly oxidized to an aldehyde with sodium periodate $\left(\mathrm{NaIO}_{4}\right)$ in aqueous solutions. As shown previously, the oxidation depth is dependent both on the concentration of the oxidant as well as the oxidation time. Further investigation has shown the dependence of hydrogel oxidation on the reaction temperature.
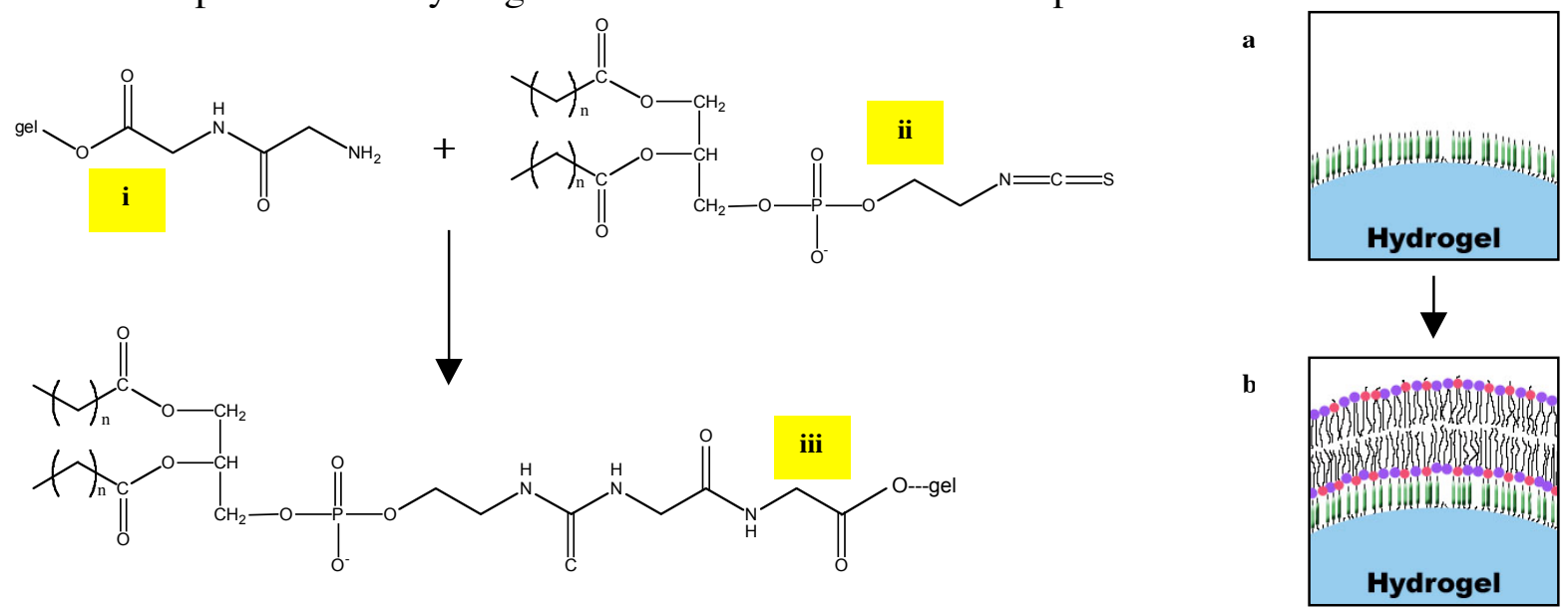

Figure 1.23. Lipid bilayer formation should proceed with one step. After the gly-gly tether attached to the hydrogel is deprotected to yield the free amine $(a, i)$, the $\mu g$ el is incubated with vesicles $(a q)$ that were made with some ratio of isothiocyanate-functionalized phospholipids (ii). Since the reaction between an amine and an isothiocyanate to create a covalent urethane linkage is reported to occur within minutes in the presence of water, this reaction should occur simultaneously to the vesicle fusion process, ultimately creating a phospholipid bilayer covalently tethered in some locations to the surface of the $\mu g e l(b, i i i)$.

Considerably longer half-lives were observed for $\mu$ gels modified with $n$-alkyl fatty acid layers with $n \geq 2$ carbons, as these $\mu$ gels significantly retarded ion penetration. However, the half-lives of $\mu$ gel expansion did not increase as the fatty acid chain lengthened, suggesting that the utilization of a hydrogel substrate involves additional factors (e.g. pinhole defects) that influence the permeability of the fatty acid coatings to a greater degree than the alkyl chain length [6]. By submerging the microchannels in a water bath of the desired temperature, the gels are capable of further tuning of the oxidation depth. As shown in Figure 1.25a and 1.25b, increasing the temperature of the water bath or concentration of $\mathrm{NaIO}_{4}$ can induce larger oxidation depths. The main goal of this work is to find the conditions that will provide the most oxidation, localized at 
the surface of the hydrogel. This would provide a thin coating of a reactive functionality (aldehyde) capable of conjugating biomolecules.
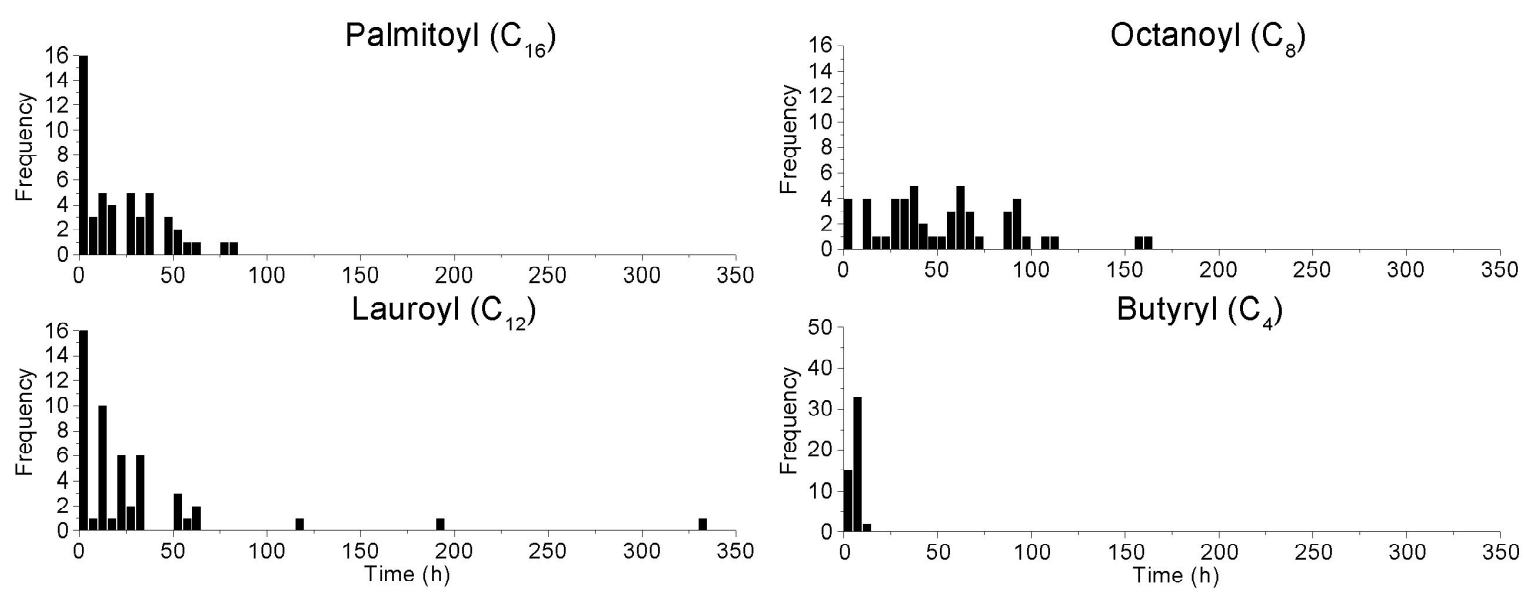

Figure1. 24. Representative histograms of the frequency of half-life values for $\mathrm{pH}$-sensitive ugels modified with saturated n-alkyl fatty acids. The number of samples with a half-life within the specified time interval (i.e., 0-5h) is indicated in each column.

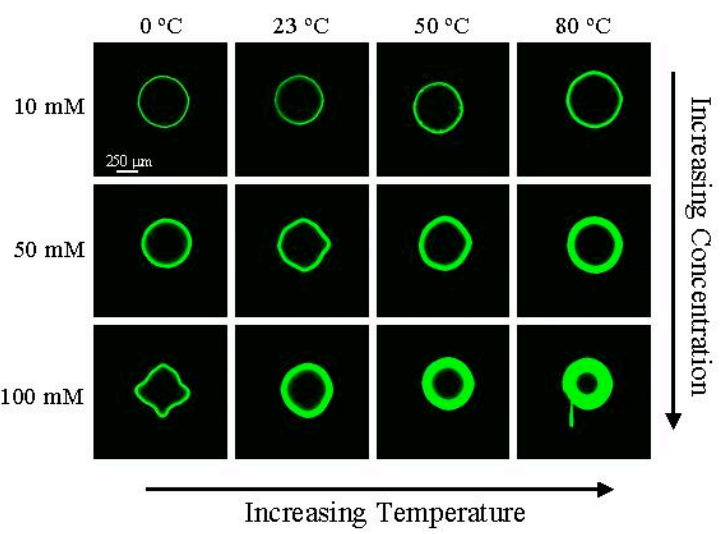

(a)

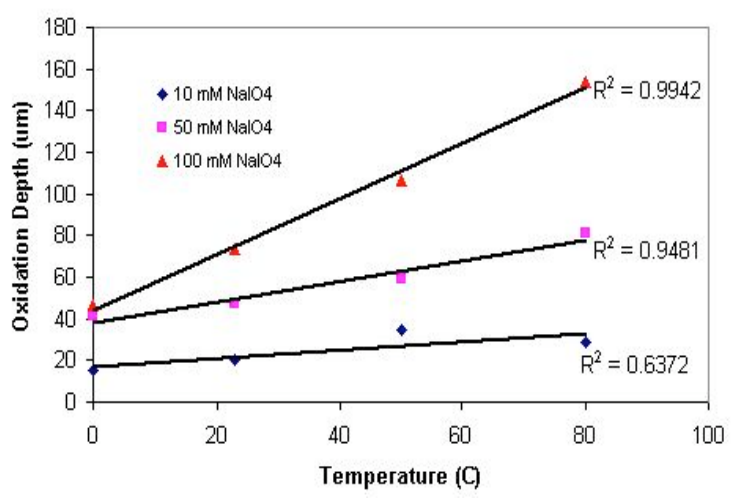

(b)

Figure 1.25. Confocal images of 12 hydrogel posts oxidized at several concentrations (10, 50, and $\left.100 \mathrm{mM} \mathrm{NaIO}_{4}\right)$ and different temperatures $\left(0,23,50,80^{\circ} \mathrm{C}\right)$ for 10 minutes (a). A graph showing the hydrogel oxidation depth (average value of each side of the hydrogel) as a function of temperature (b). These results indicate that oxidation depth increases with increasing temperature and concentration.

To test chemoselective ligation to the aldehyde-containing surface, a decyl-aminooxy molecule was synthesized. The aminooxy functionality has been found to be a selective partner of an aldehyde for chemoselective ligation. A $1.1 \mathrm{mM}$ decyl-aminooxy / $\mathrm{pH}=2.75$ buffer was 
introduced into a microchannel containing an oxidized hydrogel. The decyl-aminooxy molecules conjugate to the aldehydes displayed on the surface to create a hydrophobic ion barrier between the inside and outside of the hydrogel. To test the new ionic barrier, a decyl-aminooxy modified hydrogel was introduced to a $\mathrm{pH}=7.25$ phosphate buffer. While the non-modified control hydrogel swelled quickly, little swelling was observed in the decyl-aminooxy modified hydrogel. After 24 hours of soaking in the $\mathrm{pH}=7.25$ buffer, the ion barrier was still evident by the absence of further swelling. The introduction of a $0.1 \mathrm{mM}$ sodium dodecyl sulfate $/ \mathrm{pH}=7.25$ buffer induced the lysis of the ion barrier (Figure 1.26).
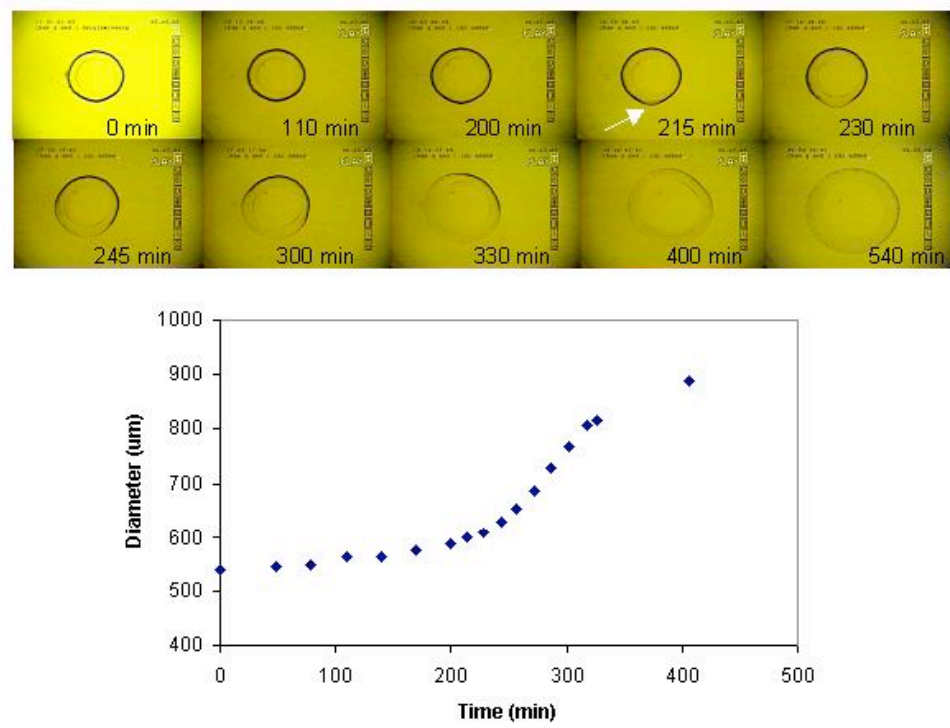

Figure 1.26. 2-Aminooxy- $N$-decyl-acetamide modified hydrogels pre-soaked in a $\mathrm{pH}=7.25$ buffer. Addition of a 0.1mM SDS / $p H=7.25$ buffer induced a breakdown of the chemical barrier produced by the chemisorbed alkyl chains. Swelling began slowly then once break point was introduced (see $215 \mathrm{~min}$ ) the swelling increased quickly.

The ion barrier properties created by acid chlorides composed of evenly numbered carbons 2-16 with saturated alkyl chains were investigated. A total of fifty runs were performed for each acid chloride. Briefly, the modification process was carried out using the same concentrations, temperature, and reaction times for each acid chloride used to modify the hydrogel. After the modification reaction, the channels were rinsed with methylene chloride to remove the excess reagents, and dried by flowing $\mathrm{N}_{2}(\mathrm{~g})$ through the channel for a minimum of $5 \mathrm{~h}$.

The ability of the fatty acid layer to establish an ion barrier, evidenced by the ability of the gel to remain contracted while exposed to an elevated buffer solution, was investigated by determining the half-life $\left(f_{D}(\ln 2)\right)$ of FAMG expansion, and the resulting data is displayed in histogram form (Figure 1.27). 

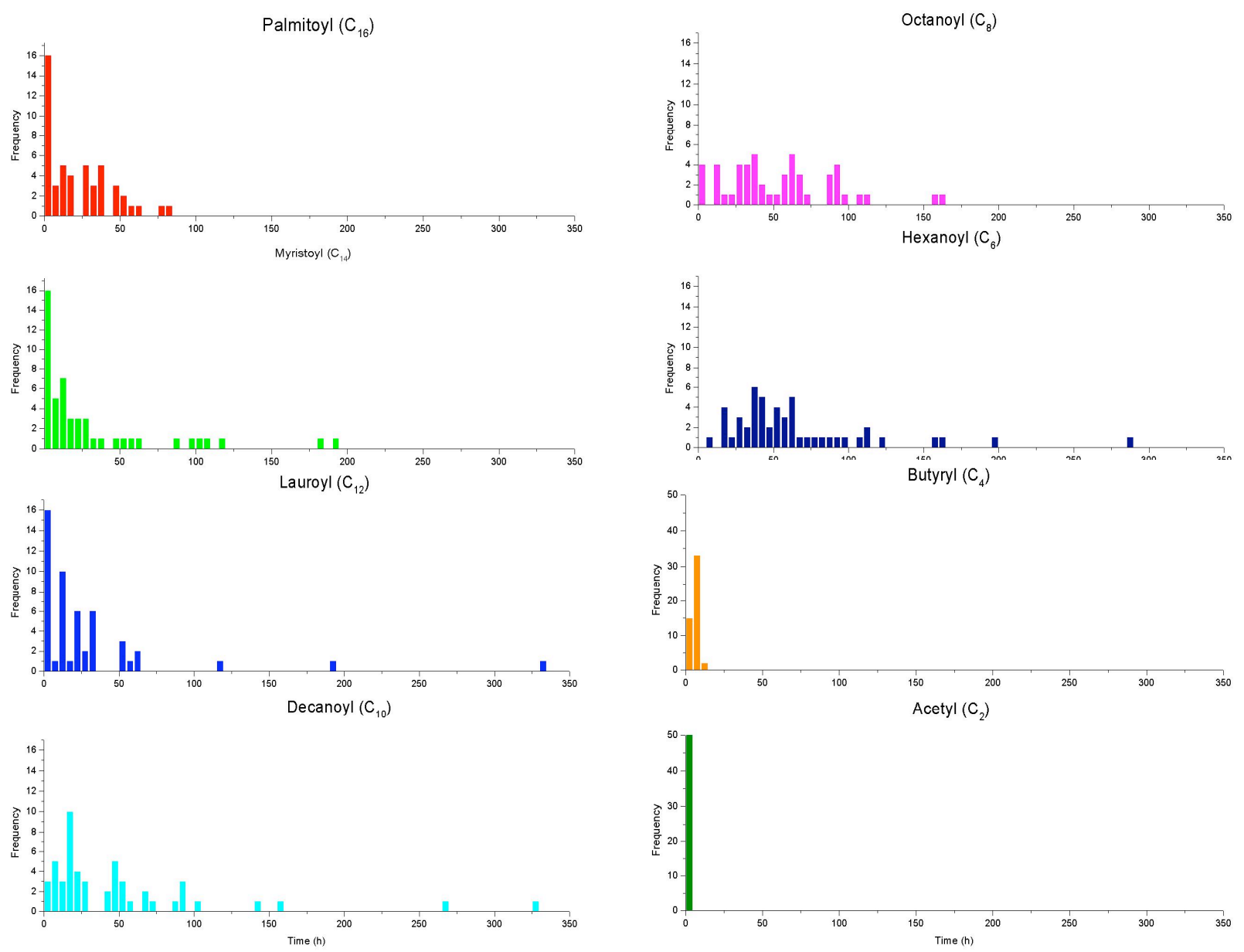

Figure 1.27. The frequency of the half-life values for the ion barrier stability of $\mathrm{pH}$-responsive hydrogels modified with acid chlorides composed of an even number of carbons (2-16), all with saturated alkyl chains. The samples that had a half-life value within the specified $5 \mathrm{~h}$ interval ( $i$. e. 0-5h) are indicated with each column. Fifty samples were tested for each acid chloride.

The ion stabilities of the gels modified with the two shortest chains, acetyl and butyryl chloride which have 2 and 4 carbons, respectively, were expected. Acetyl chloride is completely unable to establish an ion gradient and $\mu$ gel expansion proceeds uniformly around the surface of the hydrogel object immediately after exposure of the hydrogel object to the buffer solution. Initially, $\mu$ gels modified with butyryl chloride appeared to have an intact ion barrier, but in all cases this barrier was short lived and expansion was always evident in under 10h. However, the half-life for the ion stability of gels modified with hexanoyl chloride drastically increased compared to those modified with butyryl chloride, which was unexpected since the alkyl chain length only increased by 2 carbons. In addition, 49 of the 50 gels modified with hexanoyl chloride had a half-life above $15 \mathrm{~h}$. Gels modified with octanoyl chloride had a similar or slightly lower distribution of half-lives, and a more significant portion of them expanded in under $15 \mathrm{~h}$. The distribution of half-lives for gels modified with decanoyl chloride was definitely lower than those obtained for octanoyl and hexanoyl chloride, and a shift towards a lower half-life for ion 
stability as the chain length increased became apparent for the lauroyl, myristoyl, and palmitoyl chloride-modified $\mu$ gels. Oddly, the number of gels that had a half-life value of under $5 \mathrm{~h}$ was 16 for all three of these reagents.

At the onset of this investigation, it was expected that these objects would have properties similar to both monolayers on solid surfaces and lipid bilayers in solution (vesicles). Similar to vesicles and Self Assembled Monolayers, SAMs, the ion gradient established by the fatty acid layer was presumed to have a dependence on the alkyl chain length, where motions in the fatty acid tails produce transient defects where ions can pass through the insulating barrier, and the frequency of this phenomenon is lower for thicker fatty acid layers. Additionally, the FAMG ion barrier was expected to have additional properties in common with monolayers on solid surfaces due to the covalent attachment of the layer to the surface. Defects in the form of bare spots or missing chains in the layer are present on the surface after the self-assembly process for a SAM, and the consequence of these defects on the properties of the insulating layer are static; the holes change very slowly over time. Based on these two models, one would expect the manifestation of these properties on the stability of FAMGs made with various chain lengths to be two discrete average half-life values. One population would have a half-life around that of an unmodified $\mu$ gel, corresponding to FAMGs with defects in the fatty acid layer large enough to prevent formation of an ion gradient. The second population would be dependant on the length of the fatty acid alkyl chain, being longer for lengthier tails, analogous to ion transfer through transient, reversible defects caused by random chain movements. Once a certain number of ions pass through the barrier, the local underlying gel matrix expands until an irreversible defect is formed and the entire $\mu$ gel expands.

The data acquired in this study for fatty acid layers assembled on the soft hydrogel surface is inconsistent with the reported data for SAMs on solid surfaces. The ion barrier stability does not strongly correlate with the chain length. This suggests that the properties of the hydrogel matrix also play a significant role in determining the ion barrier stability of the fatty acid layer created in this study. This hypothesis will be investigated since a better understanding of the factors that influence ion barrier stability may allow us to tailor hydrogel response .

\section{Liposomes}

The feasibility of encapsulating a reducing agent in lipid vesicles to be used as a "second messenger" was also investigated. A disulfide (S-S) crosslinker was shown that could be chemically reduced which then causes disintegration of polymerized gel posts. Utilization of lipid vesicles or liposomes as a delivery vehicle adds new potential to specificity and sensitivity of future microfluidic biological detection systems. Liposomes can be engineered to carry a specific volumetric load and, more importantly, specific surface markers. Functionalized liposome surfaces can be activated by specific biochemical reactions triggered by external stimuli, which in turn cause lysis of vesicles and release of encapsulated "drugs" downstream.

The liposomes were prepared following standard techniques for producing small lamellar vesicles (SLV). A equal ratio of phosphatidylethanolamine (PE), phosphatidylcholine (PC), and 
cholesterol was used. Trace amounts of biotinylated-PE and rhodamine-PE were added for complex formation and for detection under epifluorescence microscopy, respectively. Liposomes were formed in the presence of $70 \mathrm{mM}$ tris-(2-carboxyethyl) phosphine hydrochloride (TCEP-HCl) to encapsulate the reducing agent as the "load". TCEP-HCl has been demonstrated as a strong reducing agent with good stability. The liposome mixture was finally purified by gel-filtration. Addition of CapAvidin (modified form of avidin) to purified liposomes causes formation of large liposome complexes due to biotin-avidin interaction; this effectively increases the size of the liposome in solution.

As a preliminary study, the effect of TCEP-HCl encapsulated liposome stock solution on S-S crosslinked posts was examined (Figure 1.28). The posts were incubated with unlysed followed by lysed liposome stock solutions. When straight solution is used, approximately $1 \mathrm{mM}$ TCEP$\mathrm{HCl}$ is needed to dissolve $500 \mu \mathrm{m}$ disulfide-crosslinked posts within 10 minutes. Figure 1.28 shows that TCEP-HCl encapsulated liposome stock solution is able to dissolve S-S crosslinked gel posts indicating that it is possible to load sufficient amount of the reducing agent into liposomes. Small trace of rhodamine-PE results in red background filled with small vesicles (50$100 \mathrm{~nm}$ in size). Higher liposome concentration could be employed to optimize the dissolution.

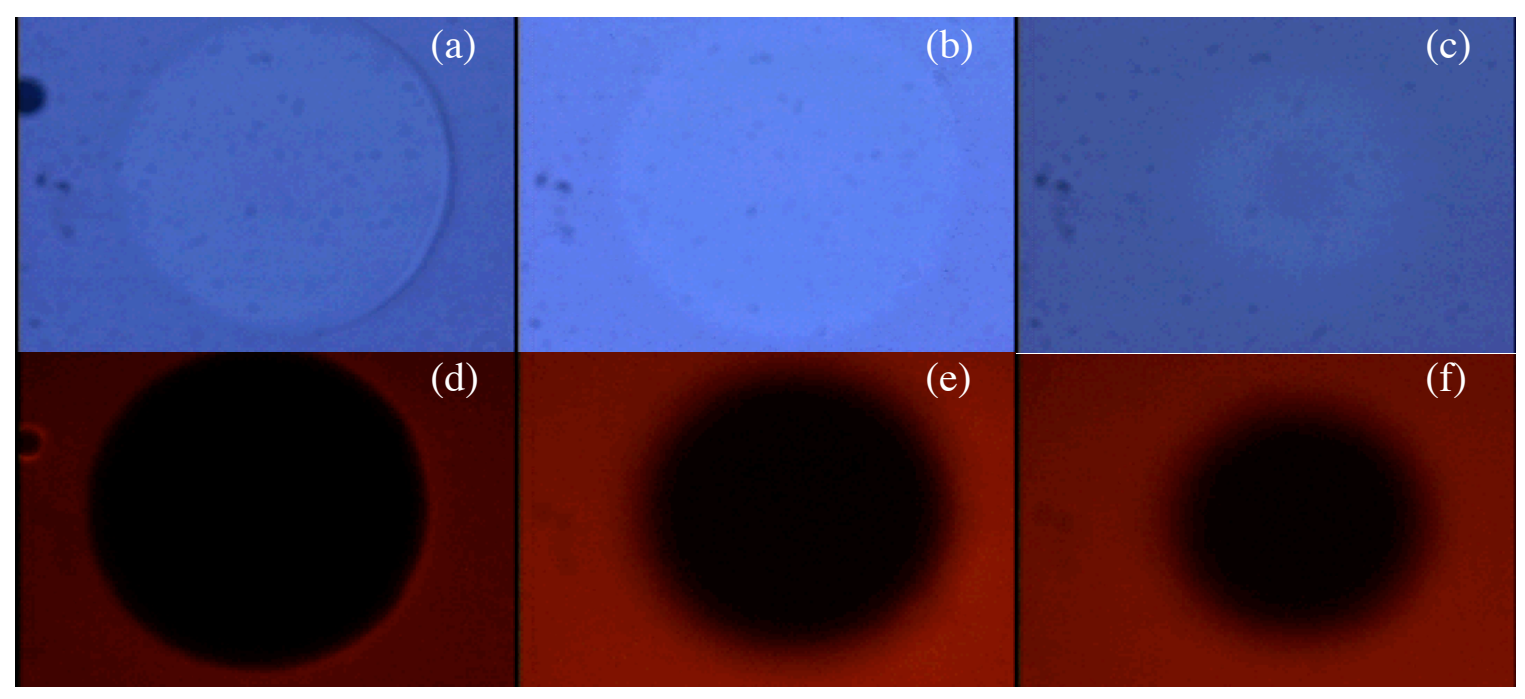

Figure 1.28. White and parallel fluorescent images of $S$-S crosslinked post taken during different exposure time to lysed TCEP-HCl encapsulated liposomes. Post with unlysed liposomes $(a, d)$, one minute after introduction of lysed liposomes $(b, e)$, and after ten minutes of exposure $(c, f)$. The exposure to released TCEP-HCl leads to dissolution of the post as seen by difficulty in edge detection and decrease in post size $(c, e, f)$. Liposomes contain a trace amount of fluorophore (rhodamine)-conjugated lipids on its surface for detection. The initial post is $\sim 500 \mu \mathrm{m}$ in diameter.

Biological detection capabilities was added to previous work on biochemical signal relay and amplification in microfluidic channels by utilizing ubiquitous and very sensitive antigenantibody interaction in conjunction with targeted lysing by complement system. 
Complement system plays a major role in innate immunity. When pathogenic antigens are detected and bound by free antibodies in blood, a group of proteins called complement is recruited to the site. This event triggers what is known as the complement cascade. The cascade, which involves multiple steps, ultimately drills holes into the membrane of the pathogen thereby lysing and destroying the cell. Artificial membranes like liposomes have been shown to trigger complement cascade in test tubes where the lysing effect is usually measured by detecting the release of encapsulated cargo into the bulk solution.

Liposomes can be functionalized by attaching specific antigenic molecules to the surface. If antibodies against the specific antigen are present in the sample, they will bind to the antigen on the liposome surface. This binding event will then trigger complement cascade, causing liposome lysis and unloading of the cargo. Lysis and unloading of liposome cargo will only occur when antigens on the liposome surface detect their target antibody in the sample. As a model, we have examined whether biotinylated liposomes can detect the presence of anti-biotin antibody and whether complement cascade could be triggered by such an event. In Figure 1.29 the filter is packed with liposomes carrying the fluorescent marker carboxyfluorescein as cargo. Anti-biotin antibody is introduced followed by complement serum. Release of carboxyfluorescein downstream of the filter indicates lysis of the liposomes. The unloading of liposome cargo implies that the surface antigens on the liposome can detect anti-biotin antibody and that such an event is sufficient to trigger complement cascade in microfluidic systems.

There is great potential for such a biochemical detection system. If the goal is to detect specific antibodies in a blood sample, both the detection and signal amplification can be triggered by the sample requiring no other reagents since complement proteins are present in blood serum. There are numerous antigen-antibody pairs of interest, and our system can be easily modified to detect different antibodies.
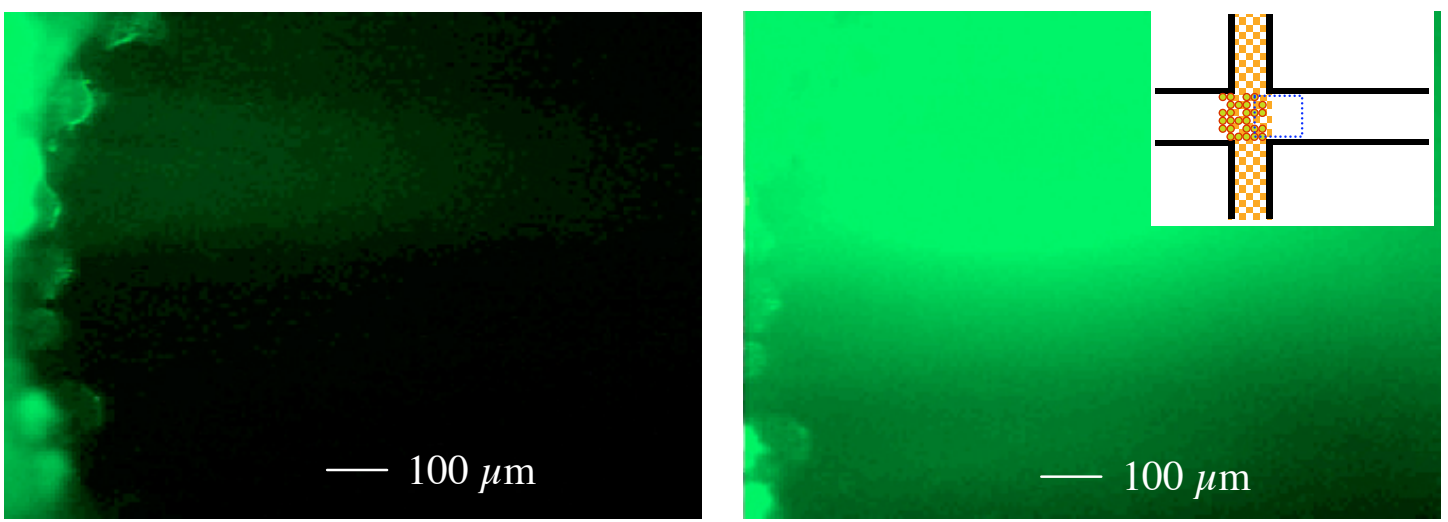

Figure 1.29. Edge and downstream of the liposome-capturing filter before and after introduction of complement serum into the microchannel. Complement cascade triggered liposome lysis and unloading of the florescent (CF, carboxyfluorescein) cargo. Such lysis is indication of specific biochemical detection event. The blue square in the inset shows the area in the channel where the micrographs were taken. 
The time needed to complete the complement induced lysis using the same liposomes was measured in test tube and data is shown in Figure 1.30. Close to $90 \%$ lysis occurs in the first 15 minutes of the reaction. This data can be used to optimize the speed at which the detection results can be obtained [7].

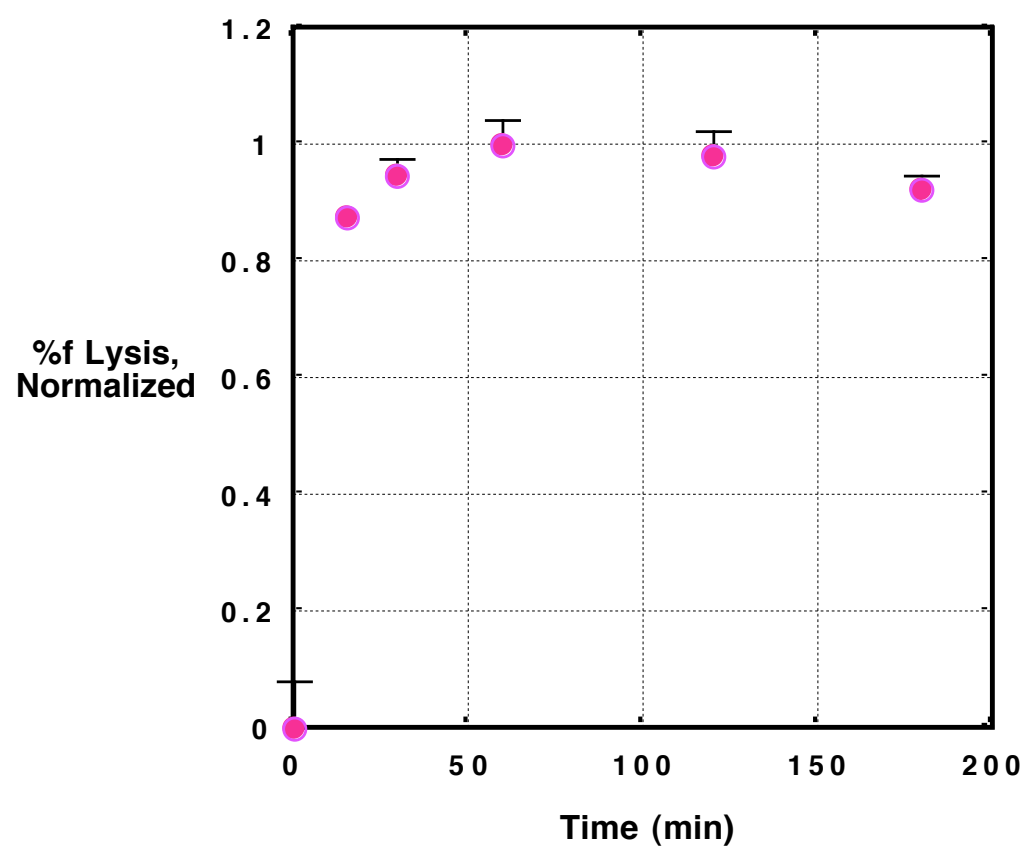

Figure 1.30. The time scale necessary for complement cascade to lyse the activated liposome is examined. Close to $90 \%$ reaction completion is achieved within 15 minutes.

\section{Liquid-liquid Interfaces}

Stable liquid-liquid interfaces have been achieved within micro-channels utilizing a photolithographic technique on self-assembled monolayers [8]. These monolayers consist of a photoactive molecule initially presenting hydrophobic perflorinated alkyl groups. Upon selective irradiation with UV light, the initially hydrophobic monolayer becomes hydrophilic, now presenting carboxcylic acid functionalities to the surface. It is this difference in surface energies that can stabilize the liquid-liquid interface of immiscible liquids. The ability to pattern and form stable liquid-liquid interfaces within a microfluidic platform allows for studies in phase-transfer catalysis, liquid-liquid extraction and interfacial polymerization.

Phase-transfer reactions involve the transport of charged ions, normally insoluble in organic liquids, to the organic phase wherein chemical reactions can take place. The transfer of these charged ions occur in the presence of a phase-transfer catalyst (PTC). These catalysts are generally quaternary amine salts possessing organic-soluble alkyl chains. The amine interacts 
with the charged ions while the alkyl chains shield the charge from the organic phase making it more soluble in the organic phase. The system studied involves an acid-base reaction between $\mathrm{NaOH}$ (aq) and 4-nitrophenylacetonitrile in Xylenes. The resulting anion of 4nitrophenylacetonitrile becomes red in color, providing a visual indicator of reaction. Addition of the PTC aliquat $(0.1 \%$ wt. in Xylenes) results in the rapid transfer of hydroxide ions to the organic phase. The PTC is critical as unsuccessful reaction was observed without the PTC. Addition of PTC, however, proved to destabilize the previously stable interface between water and xylenes. In order to stabilize the liquid-liquid interface, a Nylon-6,6 membrane was constructed between the aqueous and organic phase. A successful phase-transfer reaction was observed, indicated by the presence of the red anion in the organic phase (Figure 1.31) [9].

Chemical extractions take advantage of the differing affinity of molecules to become soluble in different solvents. Inclusion of these components into microfluidic systems provides a means to transfer molecules between phases as well as separate molecules from mixtures. Chelating metal extractions utilize the high affinity for a metallic ion to interact with organic ligands. These ligands are generally insoluble in water. As a result, when a metal ion chelates to the organic ligand, the metal is extracted from the aqueous phase to the organic phase. Dithizone is such a organic ligand, possessing a high affinity for $\mathrm{Pb}$ (II) ions. Upon chelation, the green dithizone solution becomes red, providing a visual indicator of metal extraction. A red extraction/diffusion band is observed parallel to the liquid-liquid interface and travels perpendicular to the interface (Figure 1.32).

Extractions utilizing reverse micelles have been shown to separate proteins and DNA as well as perform chemical reactions. It then is possible to envision separation/purification and chemical reactors at the micrometer scale. Extractions of this type rely on the presence of a surfactant molecule possessing a charged head group and two alkyl chains. In organic solvents these molecules form reverse micelles in which a pool of water is surrounded by the polar head groups, and the alkyl chains comprise the periphery. Molecules extracted into these reverse micelles are located generally in the water pool. The first system studied involves the extraction of $\mathrm{Co}$ (II) into hexadecane (HD). The surfactant molecule utilized is bis-(2-ethylhexyl) phosphoric acid. As the cobalt ions are extracted into HD, the solution becomes blue- indicating a successful extraction. Stable extraction was observed in systems in which one or both of the liquid streams are mobile. The stable liquid-liquid interface under flow is interesting in that extraction and fluid behavior under con- and counter-current flow can be studied. It has been shown that mass and heat transfer is more efficient when streams are flowing in a countercurrent fashion. Construction and fabrication of such systems however, is not trivial and generally rely on a solid or porous structure physically separating the two streams. Under the current fabrication techniques, a counter-current apparatus can be fabricated in which no physical barrier separates the two streams. Different extraction profiles were observed for reverse micelle extraction of $\mathrm{Co}(\mathrm{II})$. Under con-current flow, extraction was observed near the interface by the blue color in the organic stream (Figure 1.33a). The extraction profile however was not parallel to the interface, but was irregular. 


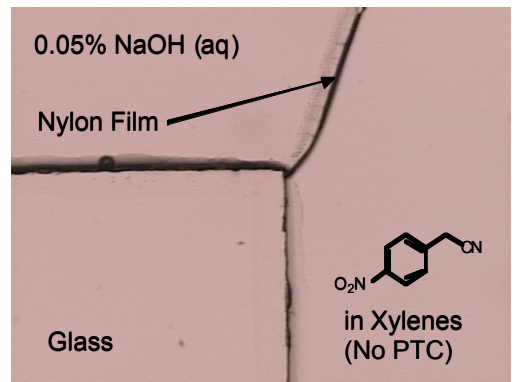

(a)

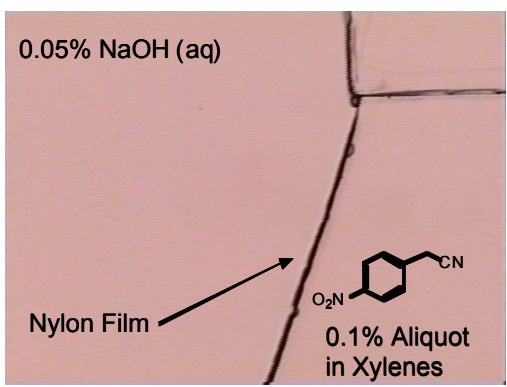

(b)

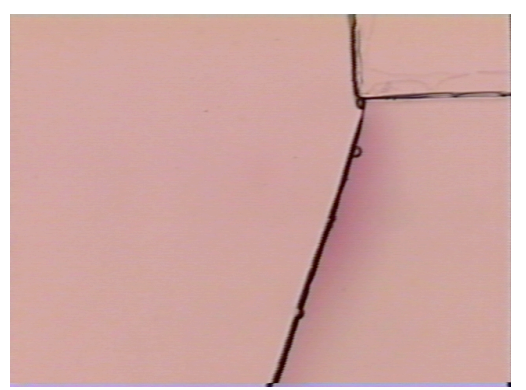

(c)

Figure 1.31. Phase-transfer reaction through a Nylon-6,6 membrane. (a) Control reaction with no PTC in xylenes phase, no red anion observed. Phase-transfer reaction through Nylon -6,6 membrane at time $=0(b)$ and at (c) 120 seconds.

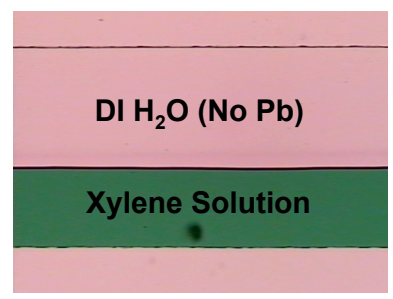

(a)

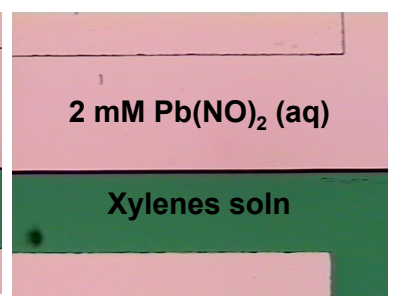

(b)

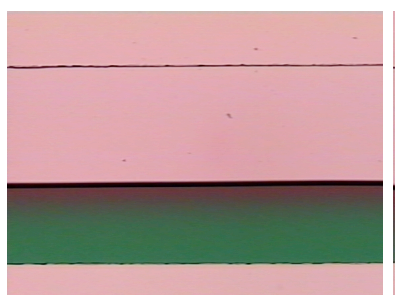

(c)

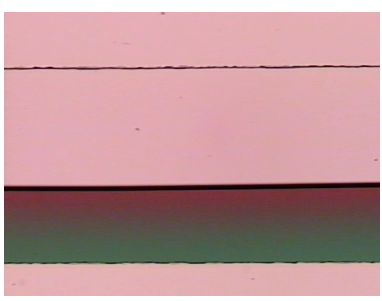

(d)

Figure 1.32. Chelating metal extration. A control reaction with no $P b(I I)$ ions in water time $=$ 5 min $(a)$. Chelating metal extraction time at $1(b), 3(c)$, and $5(d)$ minutes respectively.

The blue color generally moved in the direction of flow but the profile did not follow laminar behavior. The blue head was observed to swirl in a counter-clockwise manner as the mass moved from right to left. A constant extraction was also not observed as periods of little to no blue color was noted. Under counter-current flow, a more turbulent extraction was observed in which eddies and swirling patterns were detected (Figure 1.33b). Periods of no extraction were not observed under counter-current flow indicating more efficient extraction. The observation of turbulent flow is surprising within the microenvironment.

\section{Membranes}

Interfacial polymerization offers a wide range of applications including permeable/semipermeable membranes, solid support, and drug delivery. The interfacial polymerization of Nylon 6-6 was successful utilizing this photolithographic technique within micro-channels. ${ }^{1}$ Nylon 6-6 is a polyamide consisting of two monomeric species, an $\alpha, \omega$-diamine and an $\alpha, \omega$ diacyl chloride. If the $\alpha, \omega$-diamine was comprised of peptides, a biologically active polymer can be envisioned. These biologically active membranes lead to potential applications as solid-phase catalysis as well as biological sensors. These membranes acting as biological sensors possess several advantages within the microfluidic platform. Small amounts of reagents are needed to produce these membranes. Membranes are thin, lowering the diffusion path length of the agent to be detected (ie. large enzymes, toxins and proteases). 
(a)
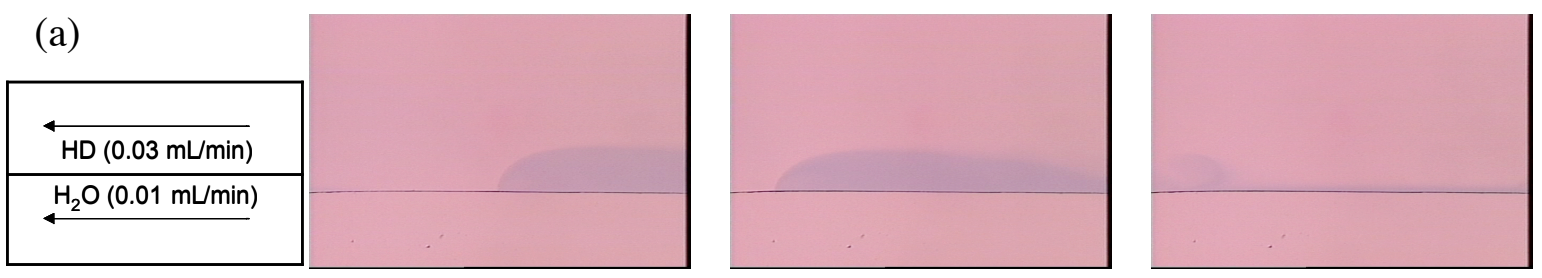

(b)
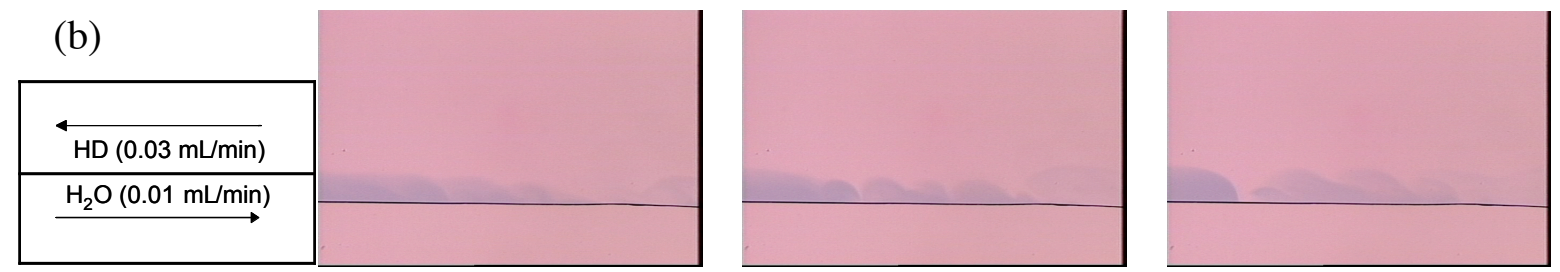

Figure 1.33. A schematic diagram (a) of con-current flow is shown for the top row of figures which show con-current extraction of $\mathrm{Co}(\mathrm{II})$ (blue) being extracted from the aqueous phase(lower) to the organic phase (upper). A schematic diagram (b) of counter-current flow is shown for the bottom row of figures that show counter-current extraction.

The first component of the biological membrane is comprised of the commercially available $\alpha, \omega$-diacyl chloride, adiptoyl chloride. Careful selection of the $\alpha, \omega$-diamine can lead to membranes that are biologically active. Amino acids are the monomeric units used in nature. In the polymer form, these peptides serve as templates, substrates, and catalysts for biological functions. The amino acid lysine contains a primary amine side chain. By choosing an amino acid sequence and terminating both ends with lysine, an $\alpha, \omega$-amine capable of polymerization can be envisioned. The amino acid sequence Ac-Lys- $\beta$ Ala-Tyr-Leu- $\beta$ Ala-Lys- $\mathrm{NH}_{2}$ was initially selected as the diamine. The amino acid residue Tyrosine (Tyr) is targeted by proteases such as chymotrypsin. Upon exposure to chymotrypsin, cleavage occurs at the C-terminal. Thus the peptide selected will break into two pieces (Ac-Lys- $\beta$ Ala-Tyr-OH and H-Leu- $\beta$ Ala-Lys- $\mathrm{NH}_{2}$. Once this peptide is incorporated into the membrane, it will break apart into smaller segments when exposed to chymotrypsin, either dissolving completely or becoming more porous. This event should be observable visibly, requiring no instrumentation for detection (Scheme 1).
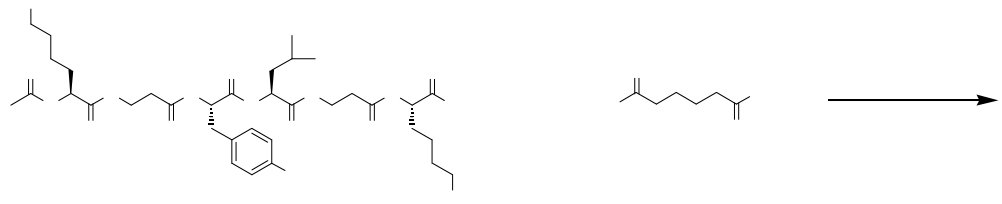

Scheme 1

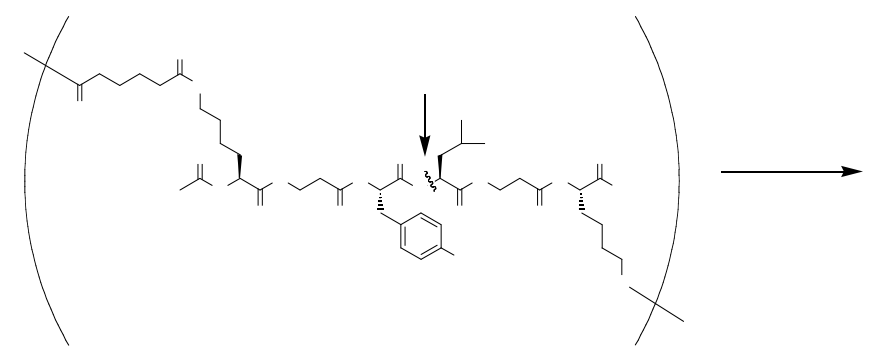


The target peptide was synthesized, and its activity when exposed to chymotrypsin was evaluated. Chymotrypsin was found to cleave the peptide at the Tyr-Leu bond, producing two peptide fragments. Interfacial polymerization utilizing the peptide sequence was successful in an H-shaped channel (Figure 1.34).

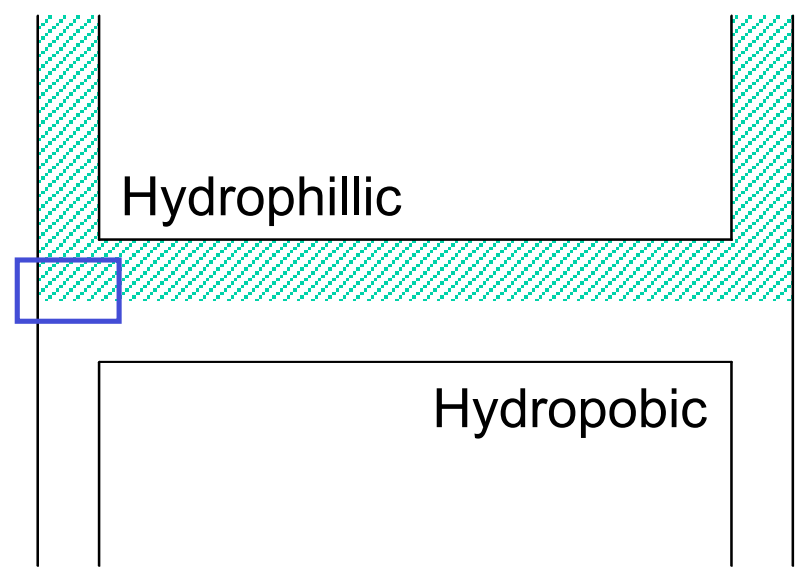

(a)

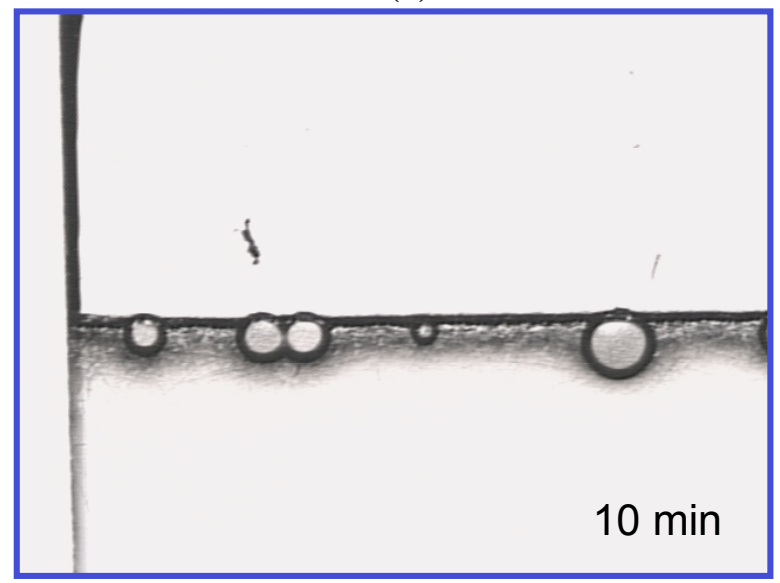

(c)

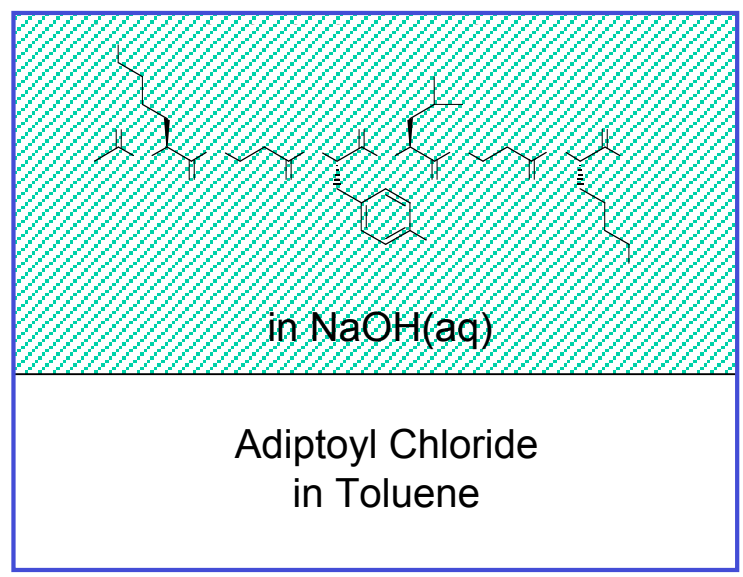

(b)

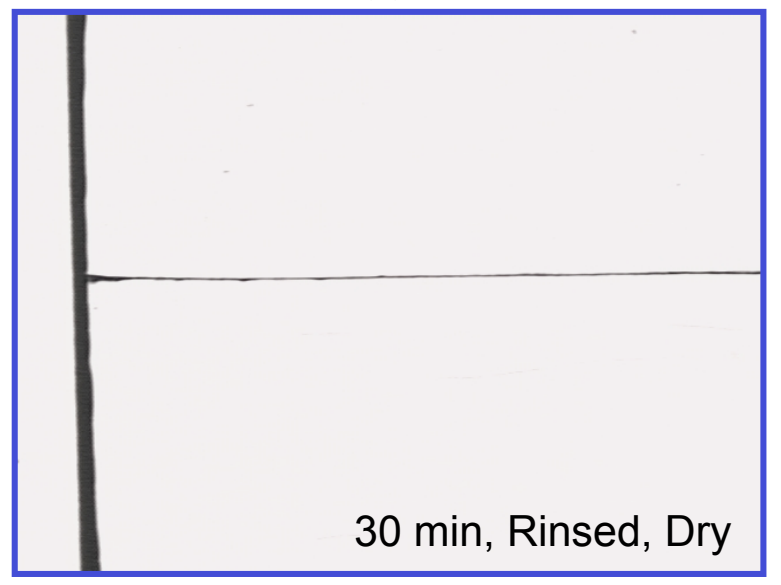

(d)

Figure 1.34. Synthesis of biologically active membranes. a) Schematic diagram of a photopatterned H-channel. The shaded area is hydrophilic and was exposed to light. The remainder of the H-channel was not exposed to light and remained hydrophobic. b) a close up view of the highlighted box in (a). The hydrophilic side of the channel was filled with an aqueous solution containing the peptide monomer. A toluene solution containing adiptoyl chloride filled the hydrophobic side of the channel. c) a micrograph of the highlighted section of the channel, after 10 min of interfacial polymerization. d) a micrograph of the membrane after 30 min of interfacial polymerization, rinsing $(\mathrm{MeOH})$ and drying under $\mathrm{N}_{2}$. 


\section{DNA Sensitive Hydrogels}

Peptide nucleic acids (PNAs) are capable of hybridization with complementary DNA strands (Scheme 2). Coupling PNA oligomers to a hydrogel may result in a system capable of detecting specific DNA sequences. Binding of the negatively charged DNA oligomers creates an ionic gradient and may result in hydrogel swelling. Potential applications are toward DNA/RNA sensing, sequencing, and PCR optimization.

Investigation toward the covalent linking of these PNA oligomers to the outer surface of the hydrogel has lead to the development of a hydrogel capable of chemical modification within the microchannel. Photopolymerization of acryloylsarcosine methyl ester and crosslinker bisacryloylethylenediamine produces a chemically active hydrogel. An amine-terminated polymer can be produced upon reaction of ethylene diamine. The difunctional linker phenylenediisothiocyanate can then be coupled to the amine groups of the hydrogel. A PNA oligomer containing a primary amino group can then be covalently linked to the hydrogel presenting the reactive isothiocyanate group, producing a hydrogel containing the DNA sensitive PNA oligomer (Scheme 3).

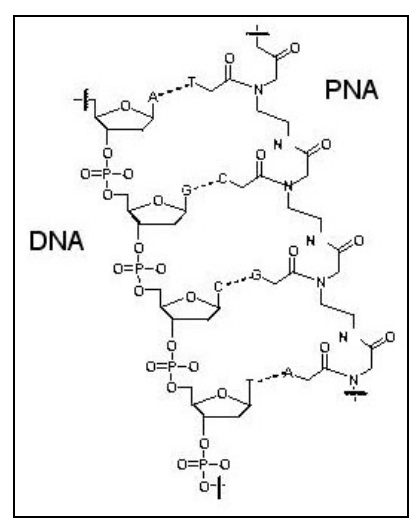

Scheme 2.

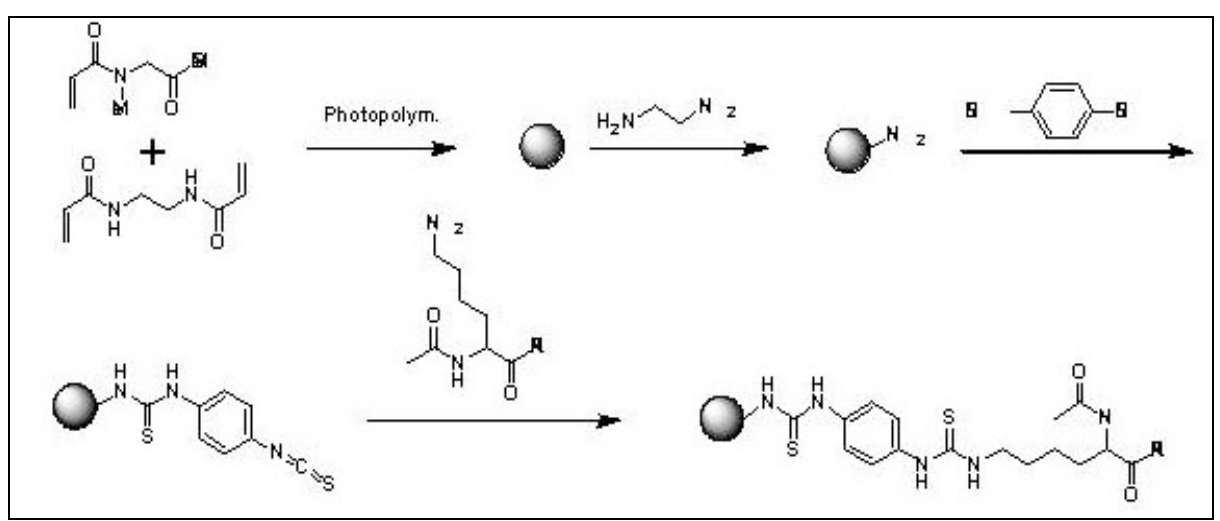

Scheme 3.

Either functional group reactive dyes or fluorophores containing similar reactive groups can visualize each step of this process. Visualization of the amine functionality on the hydrogel is accomplished by reaction with a solution of bromophenol blue dye. The blue color indicates the presence of primary amines (Figure 1.35a). Molecules containing the isothiocyanate functional group can then be covalently linked to the amine hydrogel. Visualization of this step can be accomplished by the reaction of the fluorescent probe fluorescein isothiocyanate (Figure 1.35b). Upon successful visualization of the reaction, the difunctional isothiocyanate group can be coupled to an amine hydrogel. A model reaction for the eventual PNA coupling is conducted by the reaction of a fluorophore containing an amine reactive group, Lucifer Yellow. Fluorescence on the periphery of the hydrogel indicates a successful reaction (Figure 1.35c). Upon further optimization of the reaction conditions, PNA coupling can be attempted. 


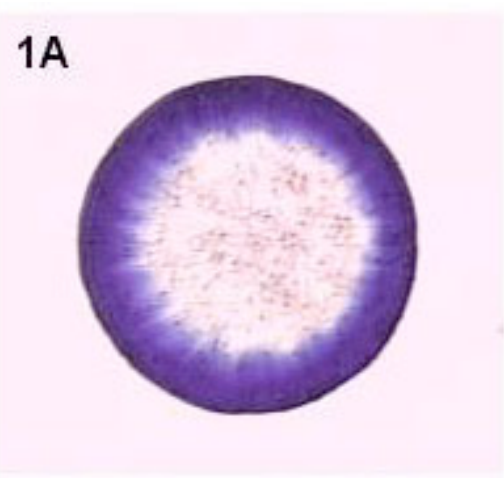

(a)

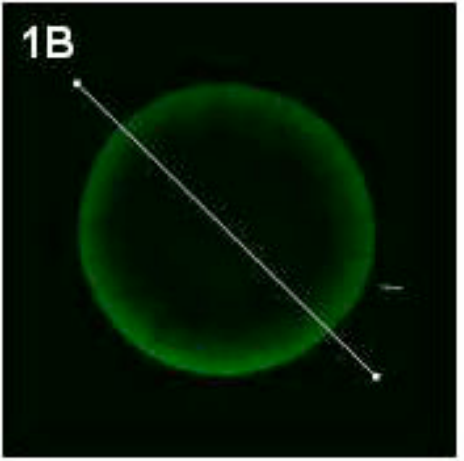

(b)

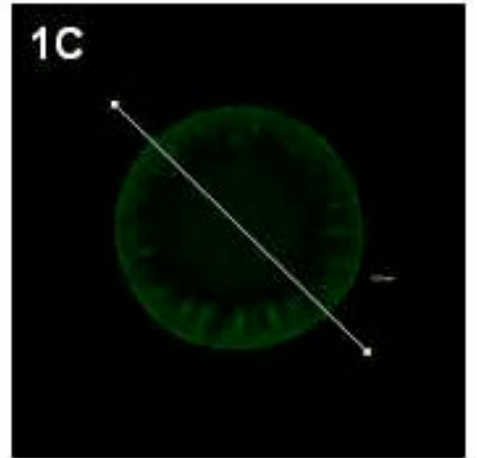

(c)

Figure 1.35. Model reaction and visualization using dyes and fluorophores. a) Ehylene diamine reaction with hydrogel produces an amine functional hydrogel. Visualization of primary amine functionality with bromophenol blue dye. b) Confocal image of a model reaction of isothiocyanate groups with the amine functional hydrogel, fluorescent dye = fluorescein isothiocyanate. c) Confocal image of a model reaction of amine groups with isothiocyanate functional hydrogel, fluorescent dye = Lucifer Yellow.

\section{Botulinum Sensitive Hydrogels}

One of the most important steps toward the development of botulinum-sensitive hydrogel is the incorporation of a peptide substrate into the hydrogel as crosslinker. To convert the peptide substrate into a polymerizable crosslinker, an efficient bioconjugation method with mild reaction condition and high yield was required. Therefore, model studies were carried out based on sulfhydryl-terminated poly(ethylene glycol) to identify the proper bioconjugation method. It was found that Michael-type addition of free sulfhydryl group to acrylamide can be carried out at room temperature in aqueous solution rapidly, leading to the desired model crosslinker with high purity (Scheme 4). In addition, the obtained model crosslinker has been polymerized into the hydrogel successfully and exhibited high crosslinking efficiency (Figure 1.36). Based on the model studies, a highly efficient bioconjugation chemistry to be used in the hydrogel formation has been determined (Scheme 5).

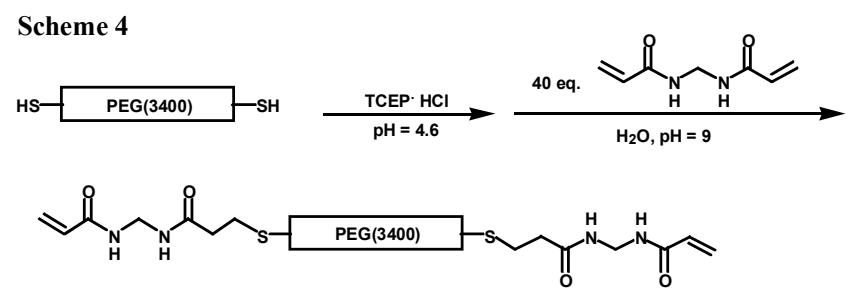




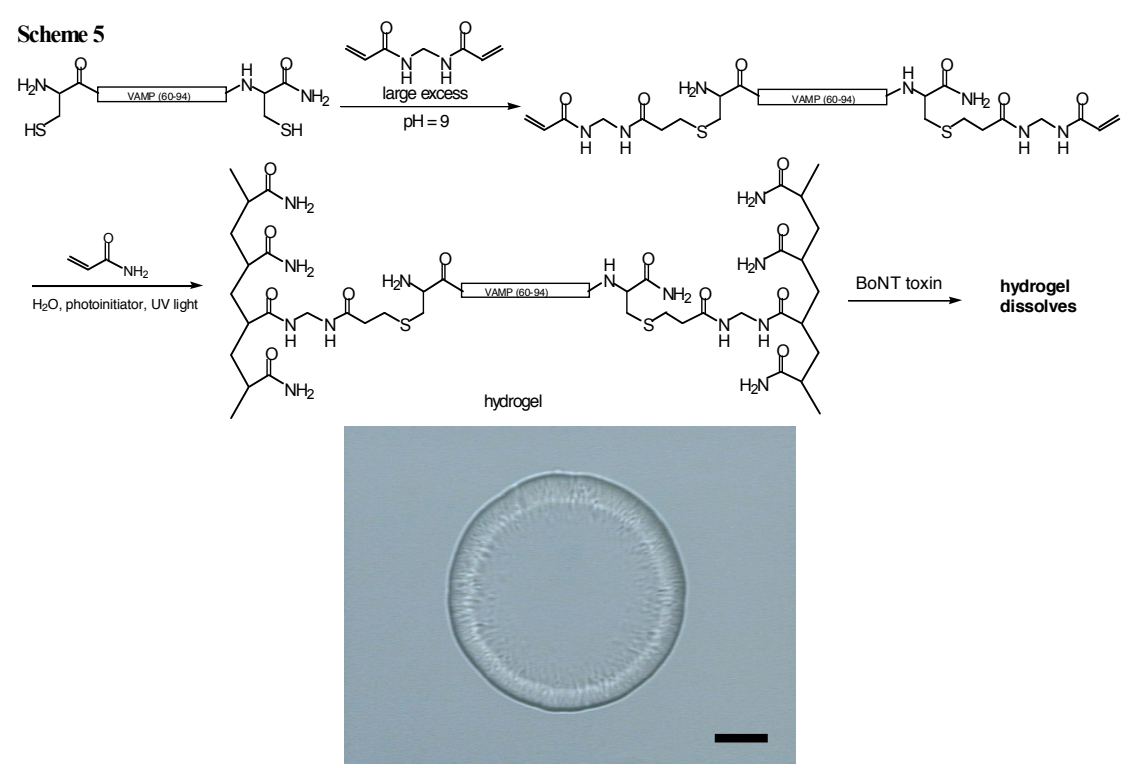

Figure 1.36. A photopolymerized hydrogel using the model crosslinker obtained from Scheme 1.

Acrylamide hydrogels crosslinked via a disulfide linkage were used as models to study the optimum condition for the proposed "biofuse" BoNT sensor. When a solution of DTT in pH 7.4 buffer is introduced into the microchannel, the disulfide-crosslinked hydrogel starts to dissolve until it completely disappears. The dissolving process was monitored at different conditions, such as varying DTT concentrations and varying flow rates, to evaluate the factors affecting the "Biofuse" sensor. It was found that the original size of the hydrogel in the microchannel and the concentration of DTT solutions play important roles, while flow rate of the DTT solution is less significant. These results provide valuable information in designing and testing future hydrogel biosensors and are shown in Figure 1.37.

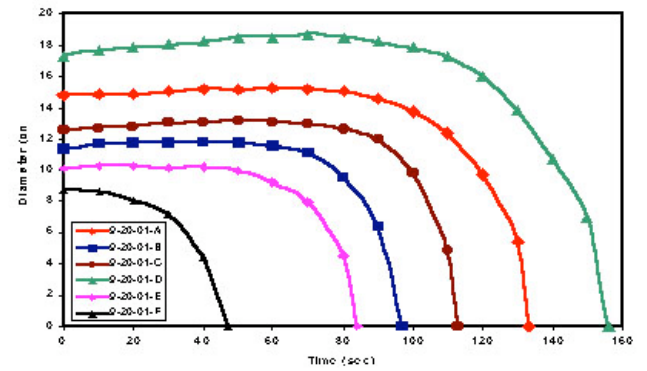

(a)

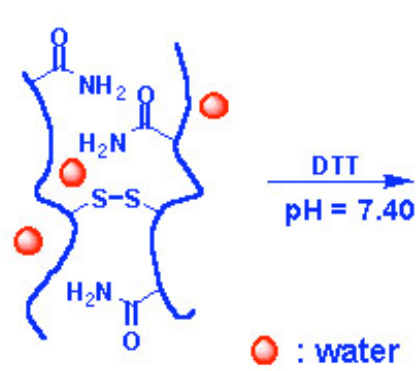

(b)

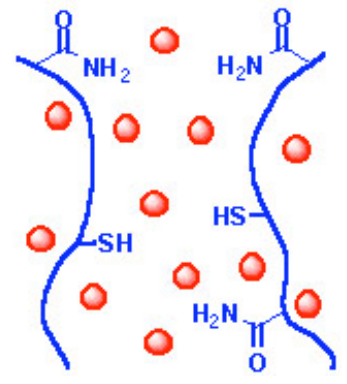

)

Figure 1.37. The dissolving curve of hydrogels (a) with different original diameter. All experiments were carried out using $50 \mathrm{mM}$ DTT solution in $\mathrm{pH} 7.4$ Tris buffer at a flow rate of $0.2 \mathrm{~mL} / \mathrm{min}$. (b) An acrylimide hydrogel crosslinked via a disulfide linkage. 


\section{Estrogen Receptor (ER) Responsive Hydrogels}

The appropriate hydrogel composition needed to be determined before an ER responsive hydrogel could fabricated. The activity of the estrogen receptor was tested with components of the hydrogel material in order to find the best combination. Acrylamide (AAm) was chosen to comprise the hydrogel matrix. Radioassay experiments showed that the ER retained a high amount of activity in the presence of AAm. Gel electrophoresis utilizes AAm, and it has a high potential for ER diffusion into and out of the matrix. AAm also swells at neutral $\mathrm{pH}$ so no extra driving force for swelling is needed. The inert crosslinker N,N'-methylene-bis-acrylamide (NMBA) was chosen for its compatibility with acrylamide, and has little affect on the activity of the ER.

The active crosslinks of the hydrogel will be formed through the dimerization of the estrogen receptor (ER), which is covalently bound to immobilized ligand. Introduction of a toxin will compete with the immobilized ligand for the binding pocket of the ER, releasing the ER from the network, and swelling or dissolving the gel (Figure 1.38). The initial approach involved conjugating a polymerizable tether to estradiol and binding the functionalized-ligand to the ER. Upon binding the ligand, the ER will dimerize forming the active crosslinker of the hydrogel. Polymerization around this crosslinker will result in an estrogen-responsive hydrogel (Figure 1.39).

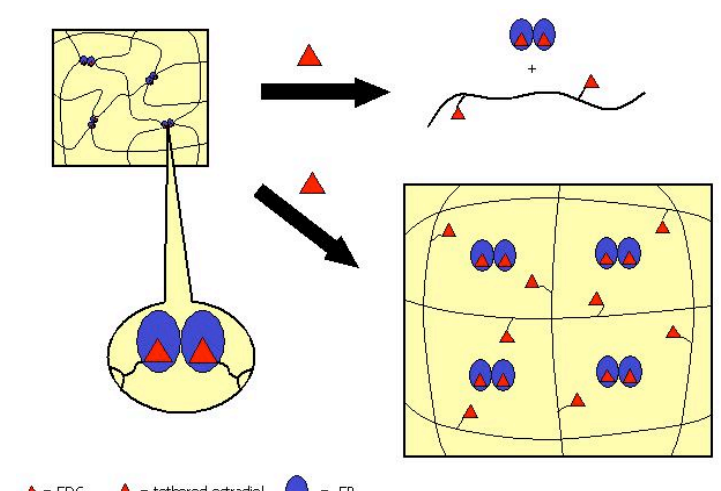

Figure 1.38. Estrogen-responsive hydrogel. The interaction of the dimerized estrogen receptor bound to immobilized ligand forms the active, noncovalent crosslinks. A toxin (EDC) will compete for the ER's binding pocket, releasing the ER from the tethered ligand, and breaking the active crosslinks of the hydrogel. 


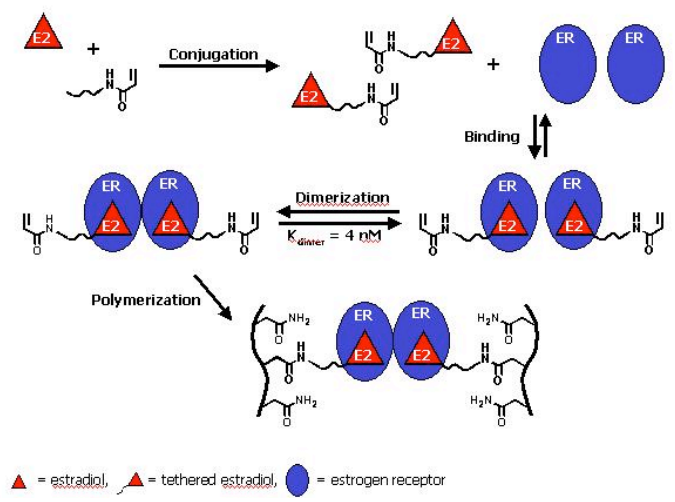

Figure 1.39. Estrogen-responsive hydrogel. Estradiol is conjugated to a photopolymerizable tether, and allowed to bind to the ER, enhancing ER dimerization. The hydrogel is polymerized around this noncovalent crosslinker.

Potential hydrogel components (monomer, inert crosslinker, photoinitiator) were first screened to determine their effects on the activity of the estrogen receptor. The components causing the least amount of reduction in ER activity were acrylamide (AAm) as the monomer, N, $\mathrm{N}^{\text {* }}$ methylenebisacrylamide (NMBA) as the inert crosslinker, 4-benzoyl(benzyl)trimethyl ammonium chloride $\left(\mathrm{BP}^{+}\right)$as the photoinitiator, and $\mathrm{N}$-methyldiethanolamine (NMDA) as the co-initiator. The concentrations of these components were then optimized resulting in a hydrogel containing $15 \% \mathrm{w} / \mathrm{w}$ AAm to water, $1 \% \mathrm{w} / \mathrm{w} \mathrm{NMBA}$ to AAm, $1.2 \% \mathrm{w} / \mathrm{v} \mathrm{BP}$ to solution and $1.2 \% \mathrm{w} / \mathrm{v}$ NMDA to solution. This monomer cocktail is photopolymerized into a $400 \mu \mathrm{m}$ sphere in $1 \mathrm{~min} 30 \mathrm{sec}$ via UV irradiation. A model study was performed with this hydrogel matrix using disulfide bonds instead of the dimerized ER as the active crosslinkers, and dithiothreitol (DTT) as the "toxin." This study demonstrated the feasibility of a responsive, poly(acrylamide) hydrogel based on a change in crosslink density. The volume change of the gel was observable with the naked eye, the gel responded at neutral to basic $\mathrm{pH}$, and the response time was rapid. A poly(ethylene glycol) tether was then conjugated to estradiol, the most active naturally occurring estrogen, at the $17 \beta$ position. The equilibrium dissociation constant of this compound was determined to be approximately $20 \mathrm{nM}$ by fluorescence polarization, a suitable value for the purposes of this research. Finally, a polymerizable unit was attached to the tethered-estradiol.

The compatibility of the estrogen receptor with the hydrogel components is crucial because the protein will be present during polymerization. Along with biocompatibility, the components must form homogeneous solutions in water, be capable of fast photopolymerization, have high gel stability, and show maximum response. In addition, the resulting gel must be able to function at neutral to slightly basic $\mathrm{pH}$, the optimal $\mathrm{pH}$ of the estrogen receptor.

Hydroxylapatite (HAP) radioassays were performed to determine the effects of potential monomers (Figure 1.40a) and inert crosslinkers (Figure 1.40b) on the activity of the estrogen 
receptor at a series of concentrations. The results from the HAP assays are summarized in Table 1.1.

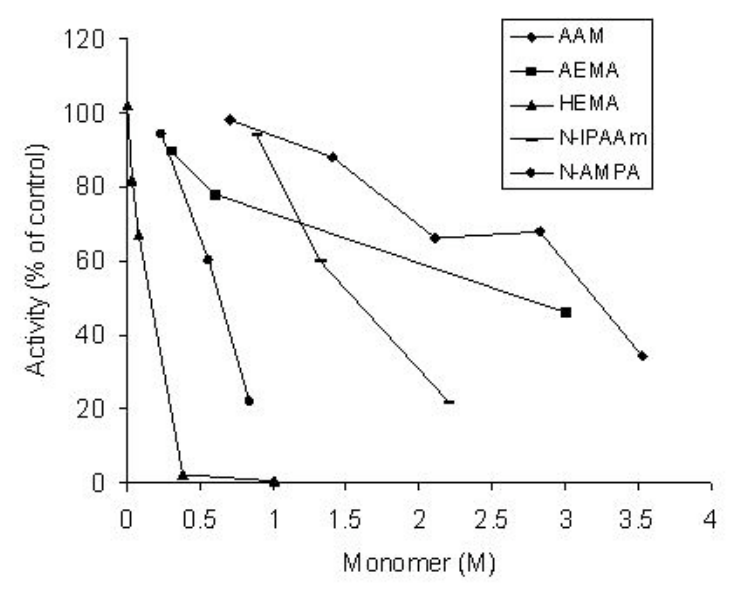

(a)

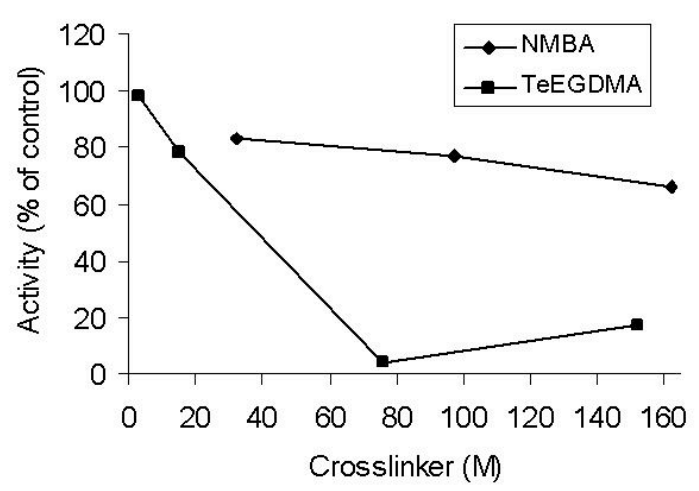

(b)

Figure 1.40. The effects of several monomers on ER activity, determined by the HAP assay (a), and the effects of two crosslinkers on ER-ES2 binding, determined by the HAP assay $(b)$.

\begin{tabular}{|l|l|}
\hline COMPOUND & M \\
\hline AAm & $\mathbf{2 . 6 3}$ \\
\hline AEMA-HCl & 2.34 \\
\hline HEMA & 0.112 \\
\hline N-iPAAm & 1.51 \\
\hline N-AMPA & 2.80 \\
\hline NMBA & $\mathbf{0 . 3 6 3}$ \\
\hline TeEGDMA & 0.052 \\
\hline
\end{tabular}

Table 1.1. Concentration of hydrogel components resulting in 50\% ER activity.

Polyacrylamide (AAm) was chosen as the major component of the hydrogel matrix because it traditionally has a high degree of biocompatibility and is widely used in biological applications such as gel electrophoresis. In addition, polyacrylamide swells in aqueous solutions at neutral to basic $\mathrm{pH}$. Since the hydrogel will exist in an initially restrained state in an aqueous environment, breaking the active crosslinks alone will cause the gel to swell. NMBA was chosen as the inert crosslinker when necessary. In addition, NMBA is structurally similar to AAm. Therefore, the hydrogel should polymerize with a uniform composition and rate. Finally, the photoinitiators tested (NMDA and $\mathrm{BP}^{+}$) were found to have no effect on estrogen receptor activity with the concentrations required.

Each component chosen to comprise the matrix of the EDC-responsive hydrogel was examined to determine the optimal concentration necessary for the formation of a stable hydrogel with fast polymerization times, short response times, and sufficiently large pore sizes to encompass high 
molecular weight proteins. Hydrogels containing 5\% - 25\% w/w acrylamide to buffer were polymerized with $1 \% \mathrm{w} / \mathrm{w}$ NMBA to acrylamide as the crosslinker with a $400 \mu \mathrm{m}$ diameter circle photomask (Figure 1.41). Gels containing at least $15 \% \mathrm{w} / \mathrm{w}$ acrylamide to buffer had good integrity, and the lower limit of these (15\% w/w AAm to buffer, $2.1 \mathrm{M})$ was chosen to comprise the hydrogel matrix. These hydrogels would exhibit the least effect on the activity of the ER, have the largest pore size, and the fastest response time.

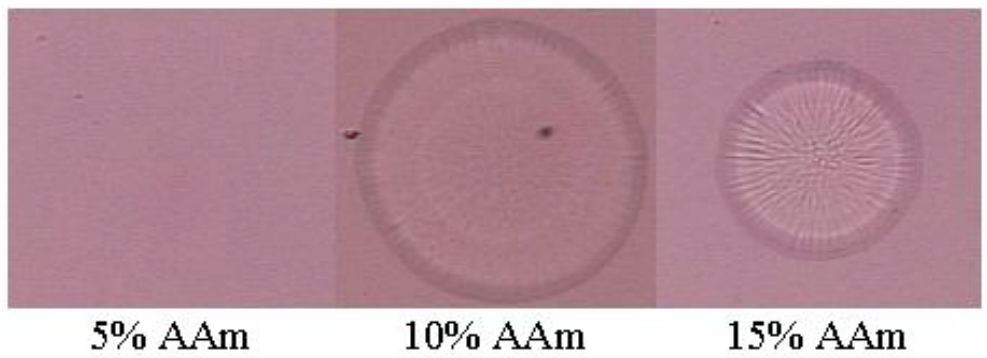

Figure 1.41. Optimization of acrylamide concentration. Hydrogels were polymerized with a 400 um diameter circle photomask. Monomer solutions with 15\% AAm formed gels with good integrity while 10\% AAm gels were unstable and 5\% AAm gels did not polymerize.

Hydrogels were polymerized with $0.3 \%$ - 2\% w/w NMBA to AAm (Figure 1.43). Although a high degree of crosslinking forms a tough gel with good integrity, the degree of swelling is low. When too little crosslinking is present, the gel is too soft to maintain its shape. At the optimum crosslinking level (lowest crosslink density that forms a usable gel), a small signal results in a large volume response. The optimum inert crosslinker concentration for an EDC-responsive hydrogel matrix was chosen to contain $1.0 \% \mathrm{w} / \mathrm{w}$ NMBA to AAm.

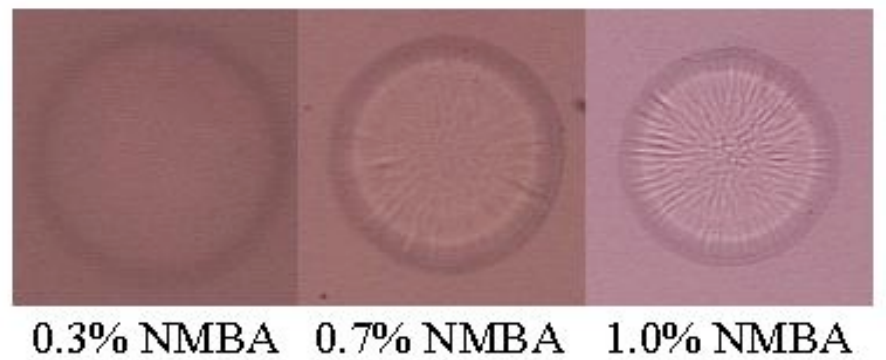

Figure 1.42. Optimization of NMBA (inert corsslinker) concentration. Hydrogels were polymerized with a 400 um diameter circle photomask. Hydrogels with 1\% NMBA contained the lowest degree of crosslinker needed to form stable gels while 0.3\% and 0.7\% NMBA gels were too soft to be functional.

The photoinitiator system, composed of $\mathrm{BP}^{+}$with NMDA, was optimized. If the photoinitiator has too low of a concentration, the rate of polymerization is decreased. If the concentration of the photoinitiator is too high, polymerization is not uniform, varying within the depth of the gel. The EDC-responsive hydrogel was chosen to contain $1.2 \% \mathrm{w} / \mathrm{v}$ of $\mathrm{BP}^{+}$to solution and $1.2 \% \mathrm{w} / \mathrm{v}$ 
NMDA to solution. These were the lowest concentrations of $\mathrm{BP}^{+}$and NMDA able to be polymerized through the $2 \mathrm{X}$ microscope lens.

The polymerization time for hydrogel formation is dependent on the type and amount of monomer, crosslinker, and photoinitiator, as well as the light intensity. The optimal polymerization time of a $15 \% \mathrm{w} / \mathrm{w}$ acrylamide to buffer gel with $1.0 \% \mathrm{w} / \mathrm{w}$ NMBA to AAm, $1.2 \% \mathrm{w} / \mathrm{v} \mathrm{BP}^{+}$to solution, and $1.2 \% \mathrm{NMDA} / \mathrm{v}$ to solution through a $2 \mathrm{X}$ microscope lens was 1 minute and 45 seconds (Figure 1.43). Shorter polymerization times resulted in gels smaller than the photomask due to incomplete polymerization. Longer polymerization times resulted in gels that were larger than the photomask due to over polymerization.

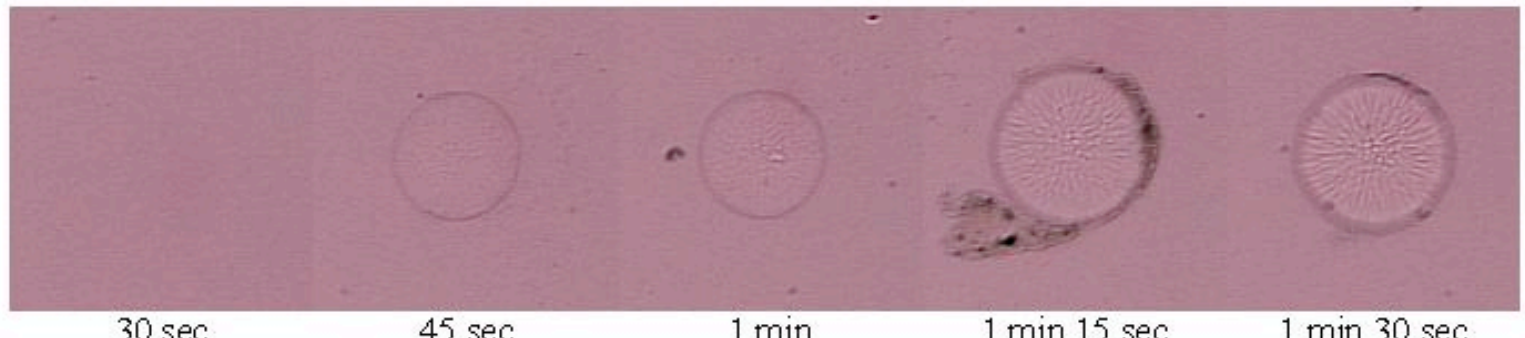

$30 \mathrm{sec} \quad 45 \mathrm{sec} \quad 1 \mathrm{~min} \quad 1 \mathrm{~min} 15 \mathrm{sec} \quad 1 \mathrm{~min} 30 \mathrm{sec}$

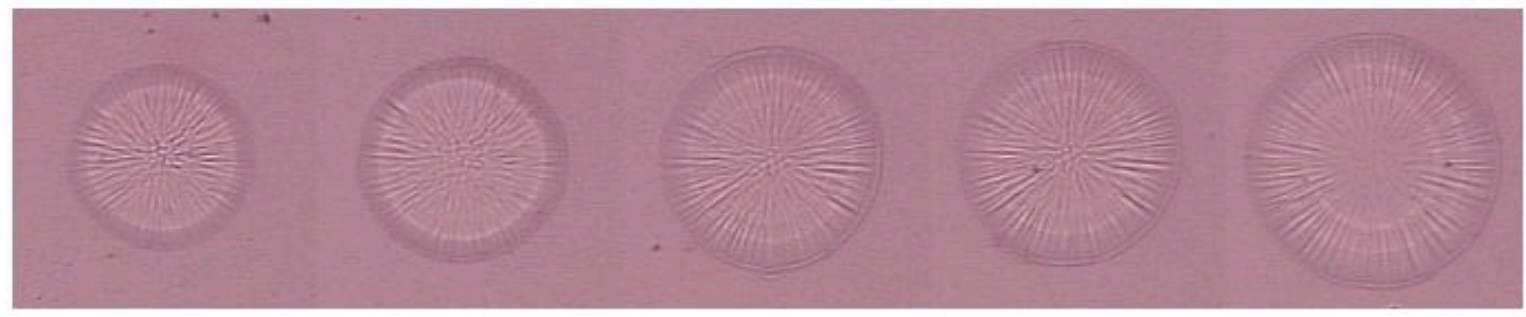

$1 \min 45 \mathrm{sec} \quad 2 \min \quad 2 \min 15 \mathrm{sec} \quad 2 \min 30 \mathrm{sec} \quad 2 \min 45 \mathrm{sec}$

Figure 1.43. Optimization of polymerization time. The gels contained: $15 \% \mathrm{w} / \mathrm{w}$ AAm to buffer, $1 \% \mathrm{NMBA}$ to $\mathrm{AAm}, 1.2 \% \mathrm{w} / \mathrm{v} \mathrm{BP}^{+}$to solution, and $1.2 \% \mathrm{w} / \mathrm{v}$ NMDA to solution. Polymerization times less than 1 min $45 \mathrm{sec}$ resulted in smaller gels (incomplete polymerization) while times greater that $1 \mathrm{~min} 45 \mathrm{sec}$ resulted in larger gels (over polymerization) when compared to the 400 um photomask.

A model study was performed to test the ability of the optimized acrylamide-based hydrogel to respond to a chemical stimulus. The active crosslinks of the gel were composed of disulfide bonds and the stimulus was dithiothreitol (DTT), a reducing agent. Upon addition of DTT to the hydrogel, the disulfide bonds break, allowing the gel to expand. Disulfide crosslinked hydrogels that did not contain the inert crosslinker, NMBA, dissolved within 4 minutes upon addition of DTT to the microchannel. Hydrogels that contained NMBA swelled to their maximum volumes within 20 minutes (Figure 1.44a). Changes in diameter of over 100\% were observed for each system (Figure 1.44b). This study demonstrated the feasibility of a responsive, poly(acrylamide) hydrogel based on a change in crosslink density. The volume change was observable with the naked eye, the gel responded at neutral to basic $\mathrm{pH}$, and the response time was rapid. These properties are important in the development of an effective biosensor. 
Tether position, composition, and length, were considered when designing the EDC-responsive hydrogel. The immobilized ligand must be able to bind to the estrogen receptor with enough affinity to crosslink the gel. However, the immobilized ligand must also be able to be displaced from the ER binding pocket by EDC's. The 17ß-position on estradiol was chosen for tether attachment because enough empty space is present in the receptor at this position to tolerate large substituents. In addition, modifying the chemical structure at the 17ß-position will not greatly disrupt ligand binding to the receptor. Poly(ethylene glycol) (PEG) was chosen as the tether because the hydrophilic PEG chain should help the solubility of the hydrophobic ligand in an aqueous environment and should extend out of the hydrophobic protein into the aqueous monomer solution.
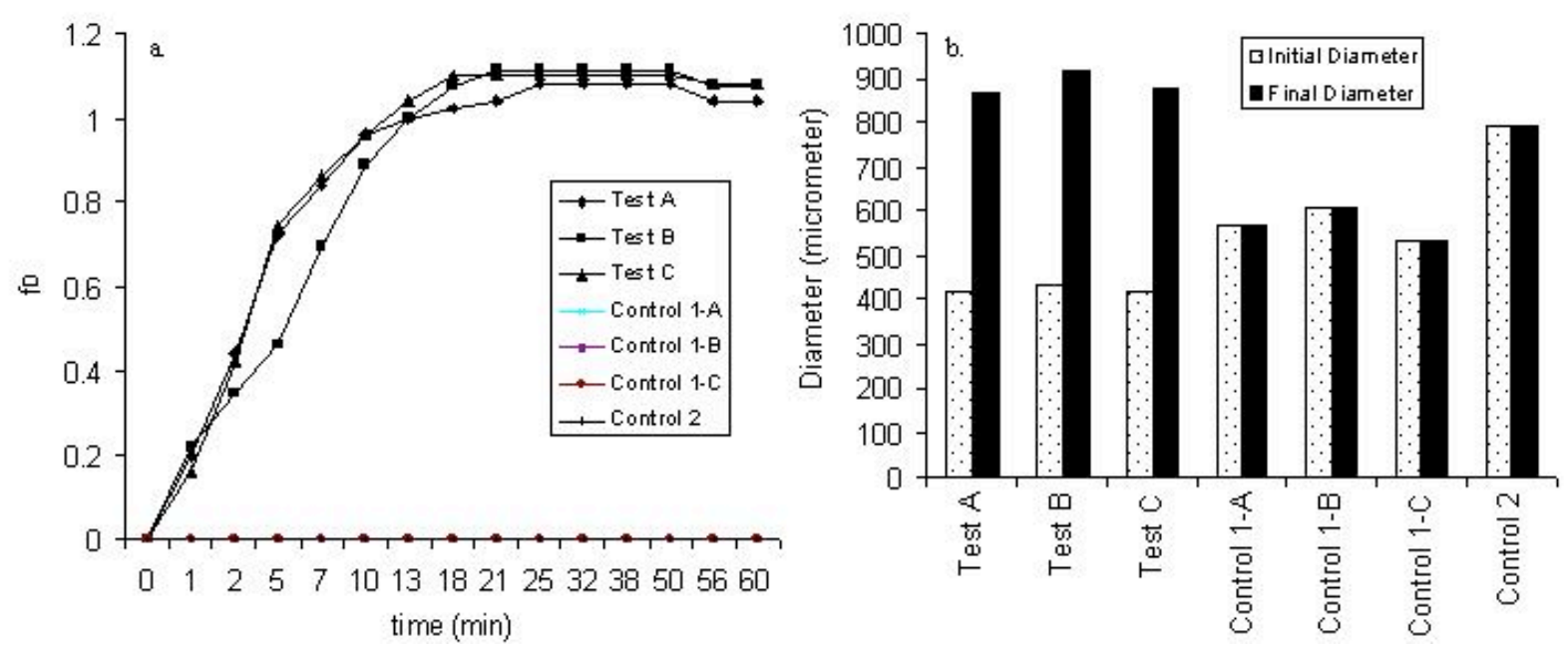

Figure 1.44. Model study of disulfide crosslinked poly(acrylamide) hydrogel. Test mixtures contained 7\% w/w CBA to AAm and 1\% w/w NMBA to AAm. Control 1 contained 7\% w/w NMBA to AAm. Control 2 contained 1\% NMBA w/w to AAm crosslinks. (a) Response of disulfide crosslinked hydrogel to DTT showed the $f_{d}=($ final diameter-initial diameter $) /$ initial diameter. (b) Degree of swelling of disulfide crosslinked hydrogel upon introduction of DTT.

A PEG trimer was conjugated to 17- $\beta$ estradiol hemisuccinate with [o-(7-azabenzotriazol-1-yl)1,1,3,3-tetramethyluronium hexafluorophosphate)] (HATU) and a photopolymerizable acrylamide unit was incorporated onto this ligand. If the tether spacer proves to be too short for polymerization into a hydrogel, longer PEG chains may easily be tethered before conjugation of the acrylamide moiety. Fluorescence polarization studies were performed to determine the relative binding affinity of the PEG-tethered ligand to the estrogen receptor (Figure 1.45). The PEG-tethered ligand was found to have a $25 \%$ relative binding affinity. 


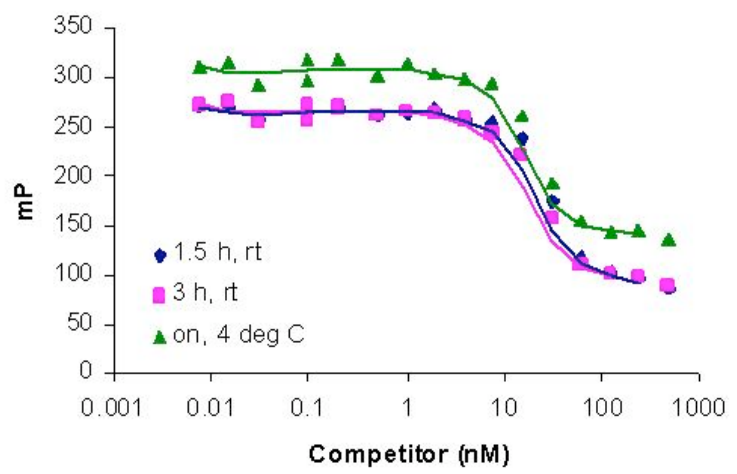

Figure 1.45. Fluorescence polarization studies were performed on serial dilutions of the competitor to determine its ability to bind to the estrogen receptor. A decrease in millipolarization $(\mathrm{mP})$ indicates that binding occurred.

This value is highly acceptable because it shows that the tethered ligand binds to the estrogen receptor with enough affinity to form a stable gel, but with low enough affinity to be competed out of the binding pocket by EDCs. The relative binding affinity (RBA) values are currently being determined for an acrylamide functionalized hydrogel. If these values prove acceptable, the acrylamide functionalized ligand will be introduced to the estrogen receptor to form an active crosslinker and will then be polymerized into an EDC-responsive hydrogel.

\section{Biofuse Material}

A model study is carried out to investigate the relationship between the volume of the hydrogel and the level of hydrogel crosslinking. Such modeling is important to future work where the sensing is dependent on the volume change of the hydrogel based on the change in the crosslinking density. In the model study, a crosslinking monomer containing a disulfide bond is polymerized into the hydrogel. Upon exposure to a chemical reagent that can selectively break the disulfide linkage in the crosslinker, a volume expansion is observed in the hydrogel. This result demonstrates the feasibility of a one-shot hydrogel "biofuse" that expands upon exposure to a biological or chemical reagent due to the cleavage of the crosslinker in the hydrogel network.

The model system being developed is crosslinked with a peptide sequence cleavable by a specific protease. When the protease is introduced to the system, it will digest the peptide crosslinker of the hydrogel, thereby decreasing the crosslink density of the hydrogel and causing it to dissolve (Figure 1.46). Towards that goal, an $\alpha$-chymotrypsin sensitive crosslinker was synthesized in the following manner. First, a tetrapeptide $\left(\mathrm{H}-\mathrm{Cys}-\mathrm{Tyr}-\mathrm{Lys}-\mathrm{Cys}-\mathrm{NH}_{2}\right)$ was synthesized on a peptide synthesizer. Second, a polymerizable crosslinker was synthesized and conjugated to the tetrapeptide through its terminal cysteines (Figure 1.47). This crosslinker, purified by preparatory HPLC, will be added to a monomer cocktail consisting of acrylamide and photoinitiator in buffer and photopolymerized into a hydrogel. 

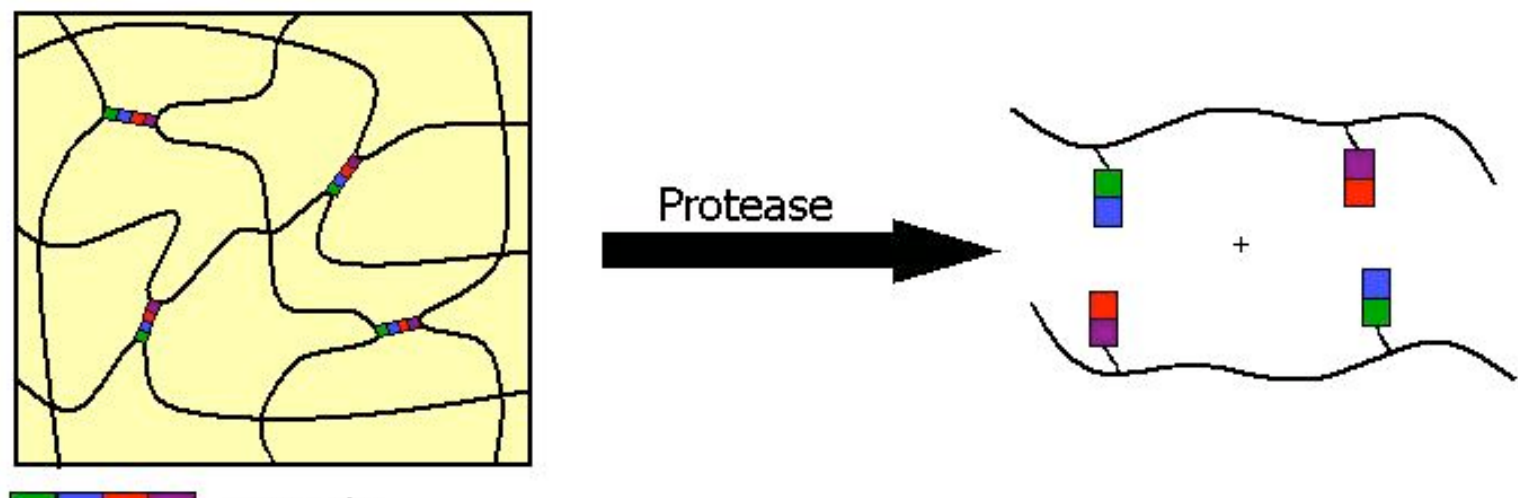

Figure 1.46. A hydrogel is shown crosslinked with a peptide sequence cleavable by a specific protease.

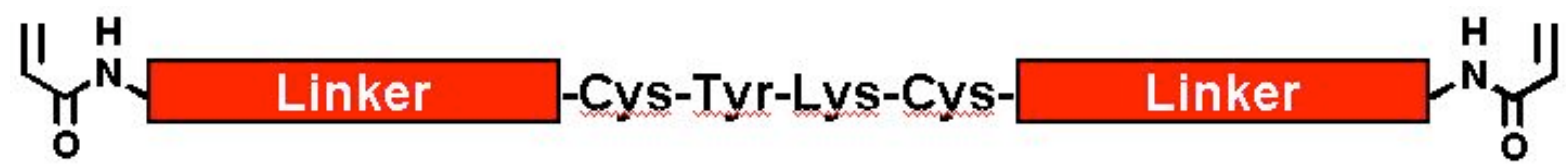

Figure 1.47. A polymerizable crosslinker synthesized and conjugated to the tetrapeptide through its terminal cycteines.

When this peptide crosslinker is incorporated into a hydrogel, the introduction of the protease, $\alpha$ chymotrypsin, will cleave the tetrapeptide after the C-terminus of tyrosine, decreasing the crosslink density of the gel and causing it to dissolve.

The ability of $\alpha$-chymotrypsin to digest the tetrapeptide was investigated via HPLC (Figure 1.48). Upon introduction of $\alpha$-chymotrypsin, the peak representing the tetrapeptide (A) as well as its oxidized, cyclic form (B) disappeared completely within $30 \mathrm{~min}$. The peptide fragment resulting from digestion is represented by peak $\mathbf{C}$. This experiment proved the feasibility of $\alpha-$ chymotrypsin to digest the synthesized tetrapeptide.

Conjugation of the tetrapeptide to linkers containing polymerizable end-groups was first attempted by reducing all of the oxidized cysteine residues and performing a Michael addition to the $\alpha, \beta$-unsaturated amides on the crosslinkers. These reaction attempts did result in the expected product, however, side-products from the addition of the reducing agent and the lysine residues to the $\alpha, \beta$-unsaturated amides also occurred. Although the side-products are relatively insignificant with the tetrapeptide, the difficulties will exponentially increase when applying this conjugation method to more complex peptides containing additional lysine residues. 


\section{Cys-Tyr-Lys-Cys $\stackrel{\alpha \text {-chymotrypsin }}{\longrightarrow}$ Cys-Tyr + Lys-Cys}

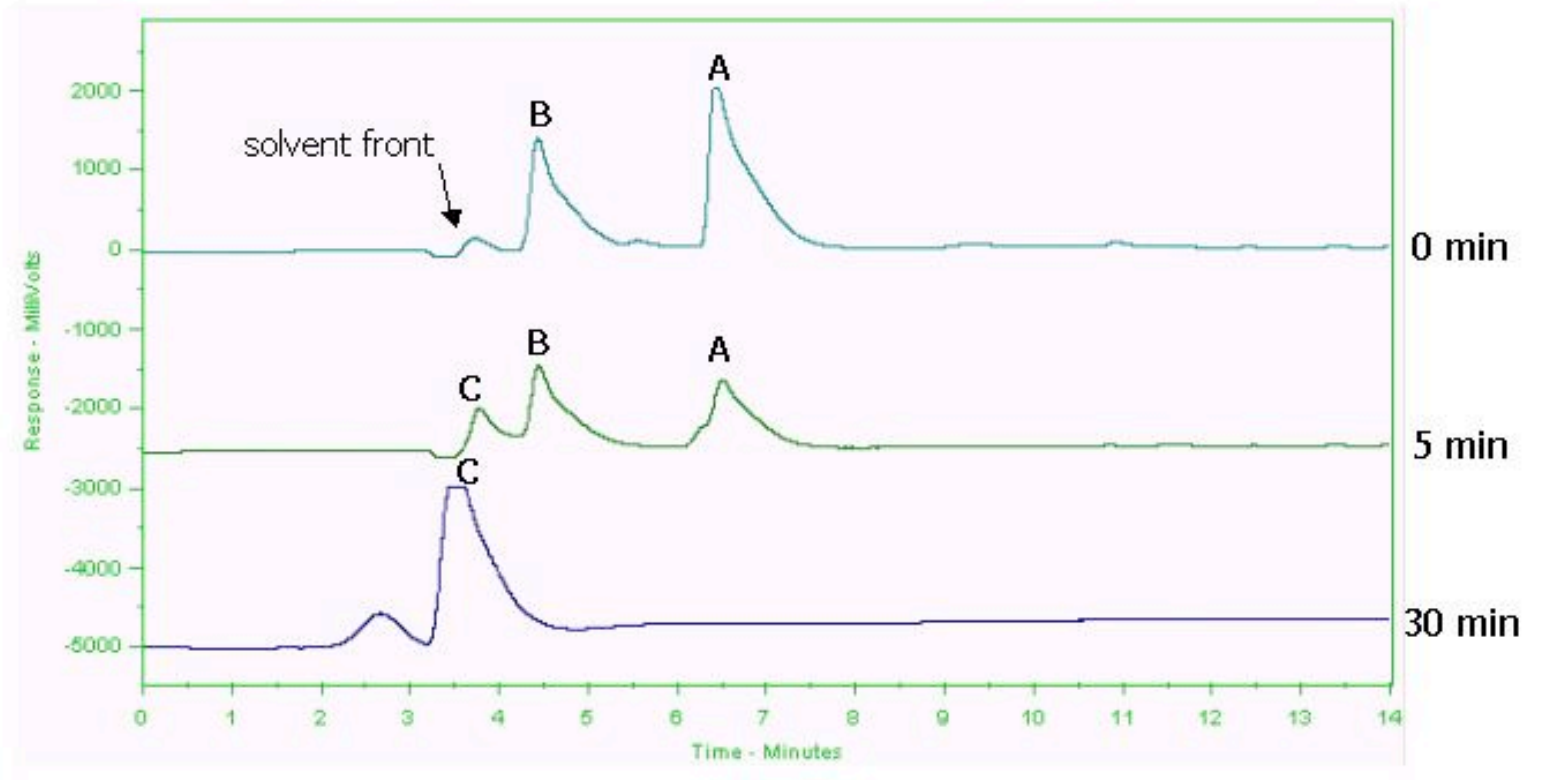

Figure 1.48. Digestion of the tetrapeptide by $\alpha$-chymotrypsin. The tetrapeptide $(\boldsymbol{A}, 2.0 \mathrm{mg})$ was dissolved in PBS (1 mL, pH 7.4). Some of the peptide oxidized into the cyclic form, $\boldsymbol{B} . \quad \alpha$ Chymotrypsin $(0.2 \mathrm{mg})$ was added to the peptide solution and HPLC spectra were taken after 0 , 5 , and $30 \mathrm{~min}$. Peptide peaks $\boldsymbol{A}$ and $\boldsymbol{B}$ disappeared with time after the addition of the protease.

The digested fragment, $\boldsymbol{C}$, was more polar than the intact peptide and eluted with the solvent front. Its intensity increased with time.

To eliminate the formation of side-products during solution-phase conjugation, the solid-phase conjugation of the polymerizable crosslinker to the peptide was employed. This technique is advantageous because an excess of reagents may be used and then rinsed away, facilitating easy purification and eliminating side reactions. A peptide containing disulfide-protected cysteine was synthesized on a solid-phase synthesizer. Upon deprotection, the cysteine was conjugated first to a maleimide derivative and then to a linker. This peptide was cleaved from the resin and is currently undergoing characterization. The second conjugation approach being explored involves the solution-phase synthesis of an unnatural cysteine residue containing a linker. This unnatural cysteine will be incorporated into the peptide via traditional solid-phase synthesis.

These techniques should eliminate the problems of side reactions with the linker and provide a generic reaction scheme applicable to larger peptide sequences. Once the conjugation reaction of the cysteine-flanked peptide to the polymerizable linker is optimized, the resulting crosslinker will be incorporated into a hydrogel. 
Since the successful implementation of a biofuse depends on a favorable environment, the focus of this work is to fabricate a hydrogel network with the ability to provide oxygen by incorporating materials that inherently trap oxygen. Progress has been made towards hydrogels with high oxygen solubility and high oxygen transporting ability. Although oxygen-dependent biological reactions have been integrated into hydrogels capable of sensing events, their effectiveness is often limited by the amount of available oxygen in the hydrogel's environment [10]. This deficiency may be resolved by designing hydrogels that are intrinsically able to trap molecular oxygen within their matrices. Fluorochemicals are known to solubilize large amounts of oxygen and are often used as blood substitutes during surgeries and transplants [11]. Thus, the incorporation of fluorosurfactants into hydrogels, should allow the hydrogels to achieve enhanced oxygen solubility and excellent oxygen transport.

pH-responsive hydrogels containing glycerol monomethacrylate and acrylic acid as the monomers, ethylene glycol dimethacrylate as the crosslinker, 2,2'-dimethoxyacetophenone as the photoinitiator, and 0, 10, and 18\% (mol to total monomer) Brij 58 as a nonionic, hydrocarbon surfactant were photopolymerized into $400 \mathrm{~mm}$ diameter cylinders (height $=180 \mathrm{~mm}$ ) containing 0,100 , and $200 \%$ water (mol to total monomer). Swelling and shrinking profiles of the mgels were obtained by introducing high and low $\mathrm{pH}$ buffer to the gels, respectively. The hydrogels exhibited a $50 \%$ change in diameter, and swelling was governed by 1 st order kinetics, while shrinking followed 1/2 order kinetics (Figure 1.49).

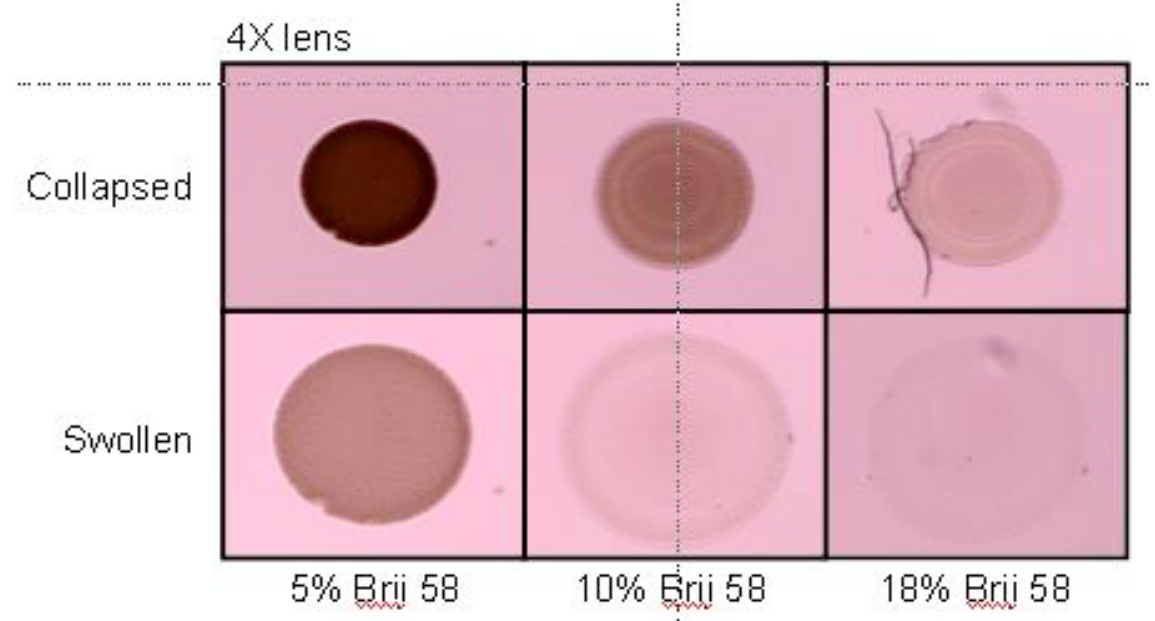

Figure 1.49. Optical micrographs of hydrogels with 5, 10, and 18\% surfactant (Brij 58) after shrinking ( $\mathrm{pH} 3.02)$ and swelling ( $\mathrm{pH}$ 10.85).

The diol moieties of glycerol monomethacrylate on the hydrogel backbone were oxidized to aldehydes, and Lucifer yellow, a fluorescent molecule, was conjugated to the gel through reductive amination. Confocal microscopy was then performed to visualize the effects of surfactant concentration on oxidation and conjugation depth. The gels containing no surfactant displayed a fluorescent ring around their perimeters, showing that oxidation and conjugation was 
localized to the hydrogel surface. Contrastingly, fluorescence was observed throughout the hydrogels that contained surfactant, with the depth of fluorescence increasing as the degree of surfactant and water concentration increased (Figure 1.50). These experiments will be repeated with a fluorosurfactant and the amount of dissolved oxygen present in the hydrogels will be determined.

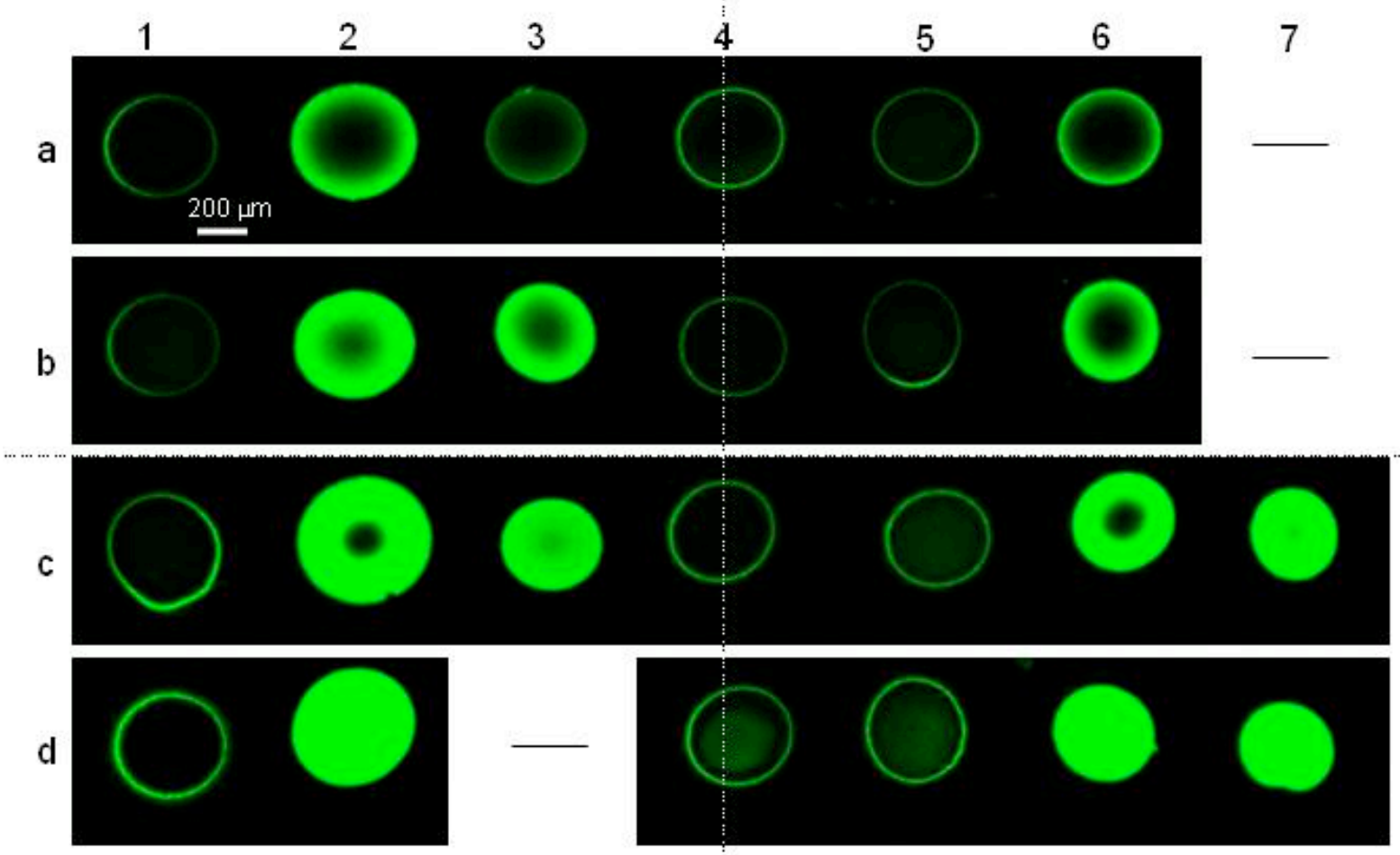

Figure 1.50. The effect of surfactant concentration on the oxidation depth of a pH responsive hydrogel containing glycerol monomethacrylate and acrylic acid. The diol moieties of hydrogels containing different concentrations of surfactant (Brij 58) and water were oxidized with $\mathrm{NaIO}_{4}$ and conjugated to Lucifer yellow to determine the degree of oxidation. 1) $0 \%$ Brij 58, $0 \%$ water 2) $10 \%$ Brij 58, $0 \%$ water 3) $18 \%$ Brij 58, $0 \%$ water, 4) $0 \%$ Brij 58, $100 \%$ water 5) $0 \%$ Brij 58, $200 \%$ water 6) $18 \%$ Brij 58, $100 \%$ water 7) $18 \%$ Brij 58, $200 \%$ water a) $10 \mathrm{mM} \mathrm{NaIO}_{4}, 10 \mathrm{~min}$ oxidation, b) $10 \mathrm{mM} \mathrm{NaIO}_{4}, 50 \mathrm{~min}$ oxidation, c) $50 \mathrm{mM} \mathrm{NaIO}_{4}, 10$ min oxidation, d) $50 \mathrm{mM}$ $\mathrm{NaIO}_{4}, 50$ min oxidation.

Previously a method of forming chymotrypsin-responsive membranes was demonstrated within microchannels. These membranes contained peptide sequences that degraded upon the introduction of chymotrypsin. Because of this success, an attempt was made to expand this work to include biologically active protein membranes. Applications of these membranes could result in events such as the detection of biological agents. 
The formation of stable liquid-liquid interfaces is possible within microfluidic channels. These interfaces are ideal for controlled membrane formation through interfacial polymerization, a property that is further enhanced if the channels are patterned with hydrophilic and hydrophobic regions. The vision of this application involved the flow of a protein containing multiple binding sites through the hydrophilic region of the microchannel, while flowing a multi-functional ligand through the hydrophobic region of the microchannel. The multi-functional ligand should bind to the protein at the interface, forming a membrane across the microchannel.

The microfluidic channels were constructed out of thin cover glass slides and then photopatterned so that one half of the H-shaped channel (the upper $\mathrm{U}$ ) was hydrophobic, while the other half (the lower $\mathrm{U}$ ) was hydrophilic. Changes in hydrophobicity were analyzed by monitoring the contact angle of the surface.

Ligand Synthesis. The Biotin-Avidin system seemed ideal for membrane formation because of the strong binding of the ligand to the protein as well as the availability and cost of the compounds. Avidin, a protein containing four biotin binding sites and a biotin dimer containing a poly(ethylene glycol) linker was used as received. A biotin dimer containing an aliphatic linker was synthesized through the reaction of biotin with an aliphatic diamine, and a three-arm biotin ligand was synthesized through the reaction of EZ link Biotin-PEO-LC-amine with tris(succinimidyl aminotriacetate) (Figure 1.51).

Attempted Membrane Formation. A solution of avidin in phosphate buffered saline (PBS) was introduced into the hydrophilic region of the microchannel. This solution was completely contained within the hydrophilic patterned boundary. A solution of the multi-functional biotin in xylene was then introduced into the hydrophobic region of the microchannel. The microchannel was placed in a dark area for $24 \mathrm{~h}$ and membrane formation was observed via microscope.

Although visual analysis through a microscope indicated the formation of a membrane resulting from the interfacial binding of avidin to each of the three attempted multifunctional biotin ligands, none of the membranes were stable enough to withstand even a small amount of pressure or channel movement. This result is most likely due to the concentration of the components tested. The previously demonstrated peptide membranes were formed from $0.2 \mathrm{M}$ peptide solutions. However, the upper limit to avidin solubility is approximately $1 \mathrm{mM}$, a difference of two orders of magnitude. Thus, the formation of protein membranes by this method has not been demonstrated to be feasible because of protein solubility. 
a)

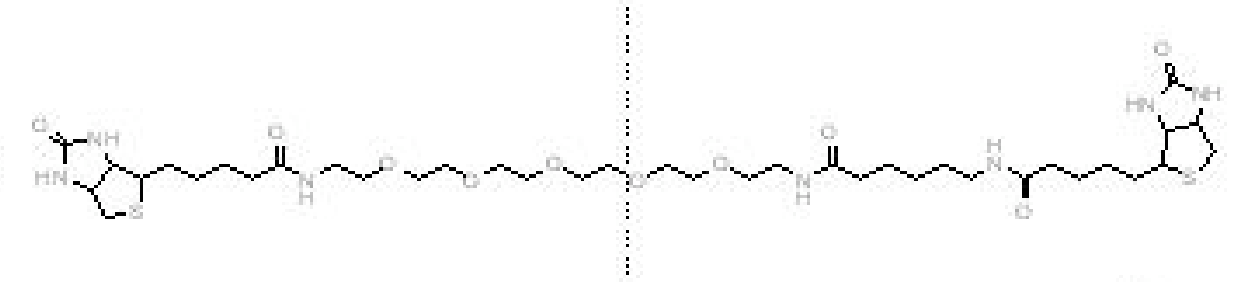

b)

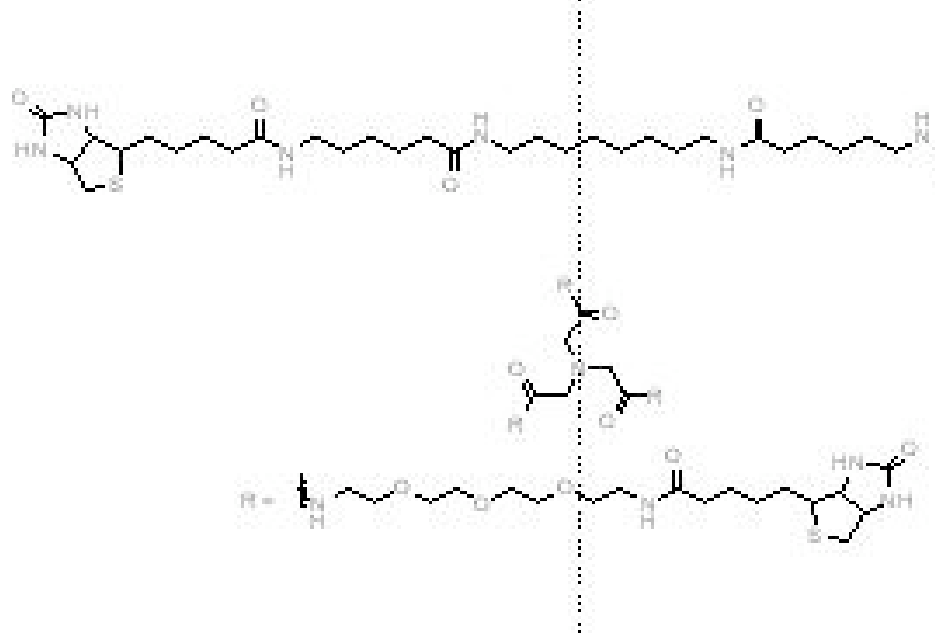

Figure 1.51. Multifunctional biotin ligands. Biotin dimers with a) a poly(ethylene glycol) linker and b) an aliphatic linker. c) Biotin trimer with a poly(ethylene glycol) linker. 


\section{Chapter 2 Components}

This chapter deals with the many components designed, developed, and demonstrated throughout the Bioflips project. The different components include; microchannels, valves, filters, sensors, mixers, membranes, and pumps.

\section{Microchannels}

Microchannels are the most basic component in the microfluidic tectonic device. Although not usually thought of as a component, the channel network is the starting block for all integrated fluidic chips. Without a fluid delivery system, devices would be ineffective.

\section{Construction Material}

In order to create the channel networks, a photodefinable polymer, referred to as construction material, is used to form the walls of the fluidic system [1]. The construction material is primarily made up of a monomer of isobornyl acrylate $95 \%$ and a crosslinker, tetraetheylene glycol dimethacrylate (TEGDMA) 5\% and a photoinitiator, dimethoxy phenol acetophenone (DMPA) $3 \%$ by weight. This material is flowed into a thin shallow cavity, a mask is placed over the cavity, and the surface is exposed to ultraviolet (UV) light (Figure 2.1). The original cavities for creating the channel networks were made from molded Polydimethyl siloxane (PDMS) bonded to glass. Because PDMS proved to be incompatible with the chemicals used to fabricate the channels, a more suitable starting block was needed.

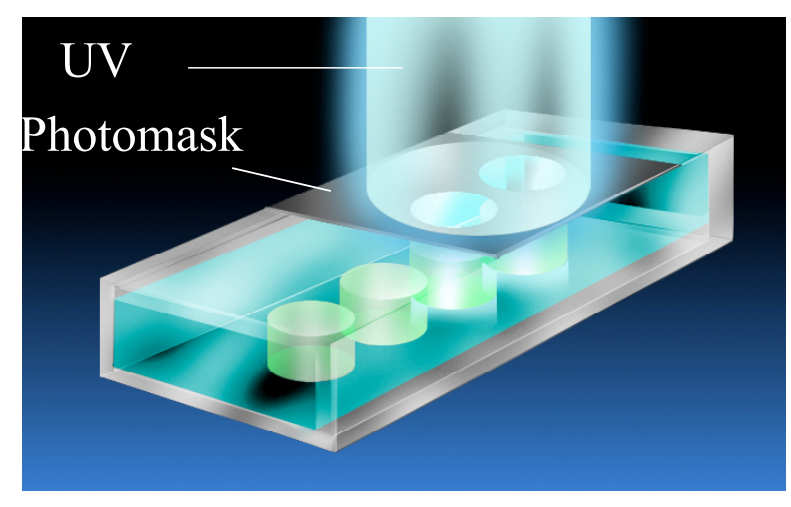

Figure 2.1. Concept photo depicting rapid channel fabrication in its simplest form.

Glass coverslips were also used since only a thin layer can be between the mask and the polymer solution. Because of capillary action and surface wetting, the construction material seeps into the gaps of the glass. When a mask is placed on top of the glass sandwich and exposed (Figure $2.2 \mathrm{a}$ ), the assembled sandwich forms a sealed cartridge complete with $\mu$ fluidic pattern. The 
device was then ready for use after flushing (Figure 2.2b). Process turnaround time was very fast, on the order of 2 minutes.

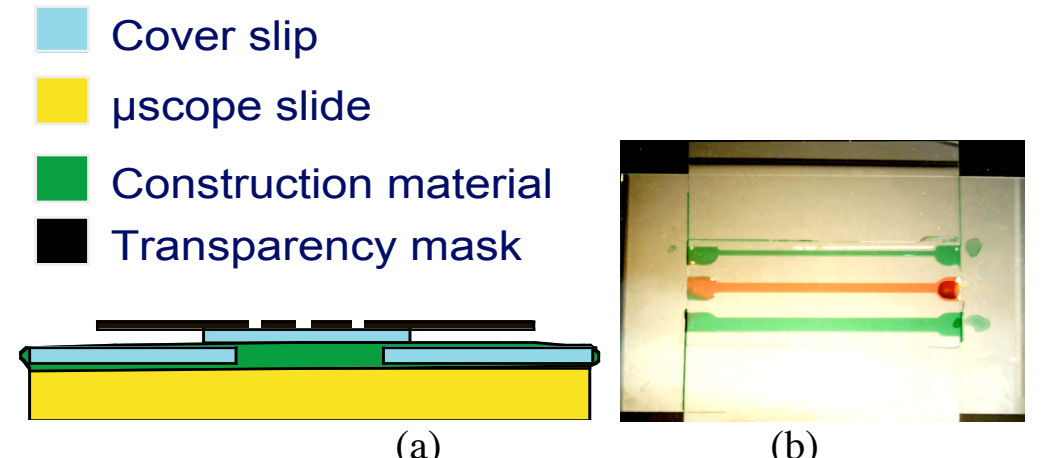

Figure 2.2. Schematic (a) of self-sealing, alignment free $\mu$ fluidic fabrication. Resultant channels $(b)$ are shown using the glass sandwiching technique. The channels were flushed with $\mathrm{MeOH}$ and filled with dyed water.

While the glass cover slips proved to shorten the fabrication time dramatically, the problem with implementing this method of fabrication process for the universal cartridge remained the connection scheme. In order to overcome the connection problem, a commercially available product was found that would serve as a suitable cavity for device fabrication.

The new universal cartridge was comprised of a polycarbonate top with 6 pre-punched holes and an adhesive gasket (commercially available from Gracebio Labs, Bend, OR) that easily attached to a microscope slide bottom. The pre-drilled holes allowed construction of three separate channels (Figure 2.3a) or one interconnected channel (Figure 2.3b). If more access holes were needed, more holes were punched into the top using a standard $1 / 16^{\text {th }}$ hole punch. This new cartridge allowed for fast construction of the cartridge cavity (30 seconds) and rapid fabrication of a microfluidic device ( 90 seconds). The process of forming the cartridge and channel network is referred to as ultra rapid prototyping (URP) [12]. Press-on connectors were designed and developed for flow through fluids (Figure 2.3b) that proved to be cheap and easy to manufacture. Multiple layers that are interconnected have also been demonstrated and are shown in Figure 2.3c.

The techniques for multi layer device construction have been extended to include interconnected layers. Previously, the layers were shown connected at the prefabricated fluid ports (Figure 2.3c), but this method limited the degree to which different layers could be connected. A conceptual drawing of a new interconnected microfluidic device is shown in Figure 2.4, and demonstrates how an integrated system can be fabricated using microfluidic tectonics. The integrated system will be used to incorporate components such as filters, pumps, valves, reservoirs, sample inputs, etc. 


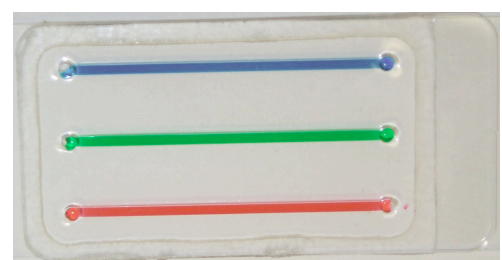

(a)

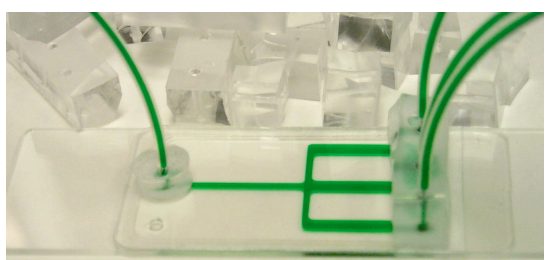

(b)

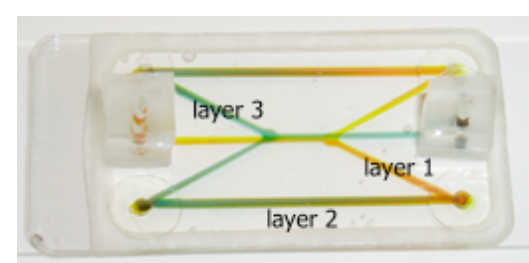

(c)

Figure 2.3. a) An example of a universal cartridge showing three separate channels, and b) a channel network inside the universal cartridge with press-on connectors is shown. c) A multiplayer device showing interconnected layers.

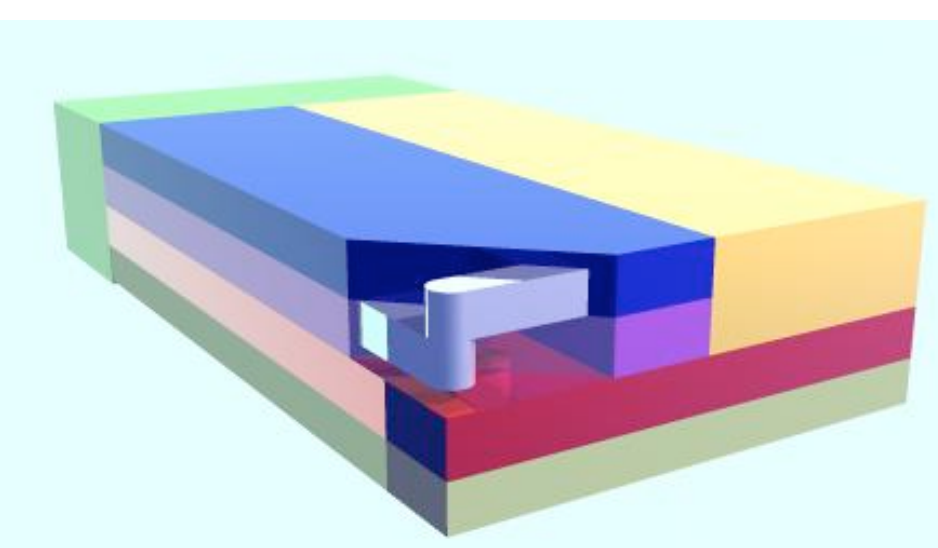

Figure 2.4. Conceptual drawing of interconnected microfluidic device. The different colors represent components needed for the integrated biodetection device.

The fabrication method for an interconnected device involves drilling or punching fluidic pathways through the polycarbonate tops [13]. The three-layer device shown in Figure 2.5a shows layers 1 and 3 interconnected to form a passive chaotic micromixer that wraps around layer 2. Although fluid passes from layer 1 through layer 2 into layer 3, the channel in layer 2 is not fluidically connected to the other two layers (Figure 2.5b, c).

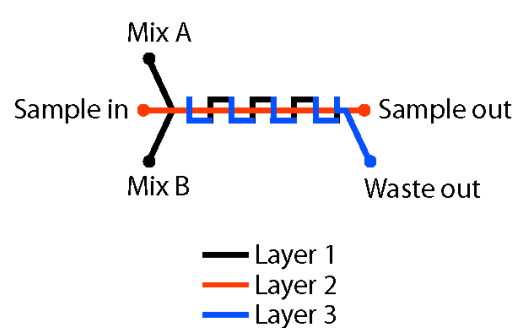

(a)

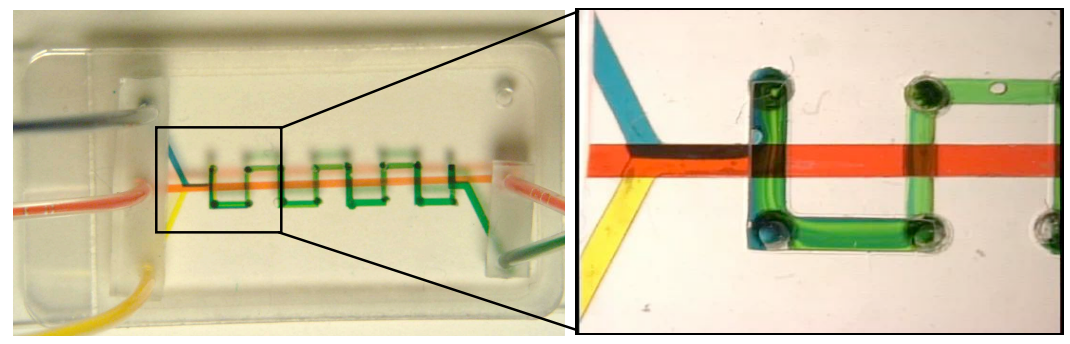

(b) (c)

Figure 2.5. A schematic (a) is shown of a three-layer device. Layers 1 and 3 are connected through layer 2 but do not interact with the channel formed in layer 2. Dyed water is shown 
flowing through the device and as the blue and yellow dyed water come together in the chaotic micromixer, the fluid streams turn green (b). The red channel flows through the device but is not connected to the other two layers (c). Channel widths are 800 microns.

\section{Alternative Material for Channels}

As microfluidic applications in cell biology move beyond diagnostic assays to long term culture and production, alternative materials will be needed. Poly (ethylene) glycol (PEG) has been widely utilized as a biocompatible polymer due to its hydrophilicity and non-fouling behavior. PEG resists protein absorption and has been used as a coating or as a polymer substrate to prevent or control cell adhesion and adsorption of proteins for over a decade. PEG has been used previously in bioMEMS-related technologies as a coating or as a co-monomer for purposes such as polymerizing cells in gels. Diacrylated PEG can be photopolymerized using the microfluidic tectonics platform $(\mu \mathrm{FT})$ and provides a more biocompatible alternative to previously used polymers. The ability to incorporate PEG as a construction material for microfluidic systems will allow the unique properties of PEG to be exploited for a variety of cell-based experiments. Examples include using in-situ polymerized porous PEG gels as selective diffusion barriers to replace media changes during cell culture, or copolymerizing with a hydrolytically degradable monomer for controlled release of biomolecules of interest. Straight channels were patterned using Polyethylene glycol (PEG) to test the patterning capabilities, with widths ranging from $175 \mu \mathrm{m}$ to $1,000 \mu \mathrm{m}$ (Figure 2.6). Good resolution of less than $10 \mu \mathrm{m}$ was achieved which is comparable to that achieved with the construction material. A valve mask was also patterned in PEGdA and shown in Figure 2.7a alongside one created in poly (IBA) Figure 2.7b. These two materials show similar capabilities although the PEGdA channels produce more rounded features.

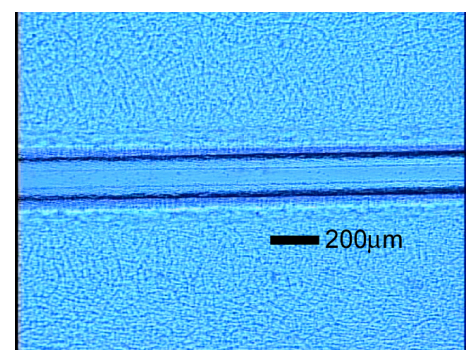

(a)

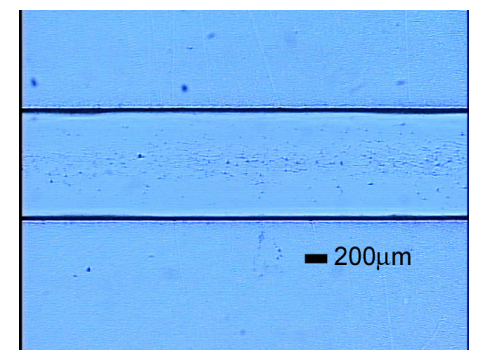

(b)

Figure 2.6. Channel walls patterned in PEG, (a) $175 \mu \mathrm{m}$ width, and (b) 1,000 $\mu \mathrm{m}$ width. An accuracy of less than $10 \mu \mathrm{m}$ was achieved with both channel widths. 


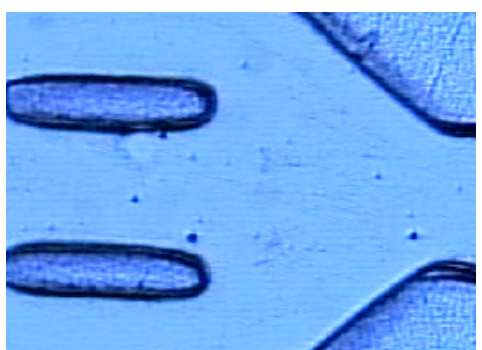

(a)

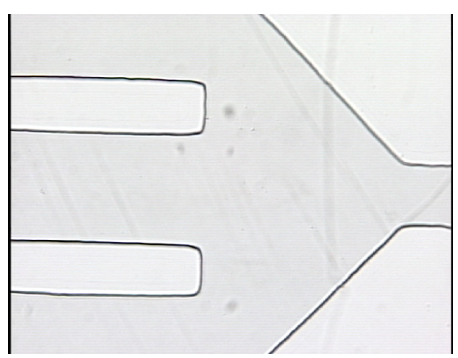

(b)

Figure 2.7. Photopatterned valve substrates, (a) made with PEG, and (b) with poly (IBA). The comparison shows features are similar in dimension but features are rounded with PEG but more optimization is needed.

The biocompatibility of this polymer will be partially dependent on the complete polymerization of the monomer while using a minimal concentration of photoinitiator. The typical concentration of photoinitiator used in these experiments was $0.05 \mathrm{wt} \%$, although lower percentages (below $0.01 \mathrm{wt} \%$ ) can be polymerized but exhibit more swelling. To verify the degree of polymerization, FTIR studies were done, comparing the spectra of the polymer with $0.1 \mathrm{wt} \%$ photoinitiator (Figure 2.8a) to that with $0.05 \mathrm{wt} \%$ (Figure 2.8b). The polymerization of diacrylates reduces the magnitude of the carbon-carbon double bond peak (shown in Figure 2.8) and is expected to decrease with increasing photoinitiator concentration as shown. FTIR measurements allow one to find a balance between concentration of photoinitiator and degree of polymerization that minimizes the cytotoxicity of the devices, while maintaining good patterning capabilities. After UV sterilization before use in cell culture, this peak is expected to decrease further.

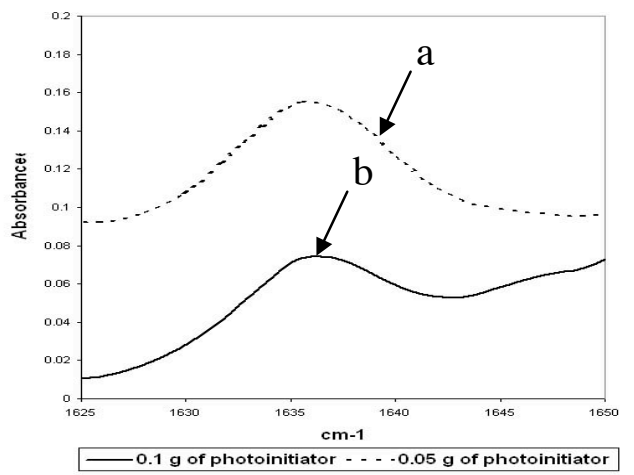

Figure 2.8. FTIR spectra of photopolymerized PEGdA, (a) with 0.1 wt\% photoinitiator and $(b)$ with $0.05 \mathrm{wt} \%$ photoinitiator. The amount of carbon-carbon double bonds decreases with increasing photoinitiator showing the increased polymerization of the PEGdA. A balance between concentration of photoinitiator and free monomer after polymerization will maximize biocompatibility. 


\section{Dissolvable Hydrogels}

We have demonstrated the use of hydrogels with breakable crosslinkers as potential biosensors. Here we show that these specifically engineered hydrogels can also be used in microfluidics as selective sacrificial structures that dissolve in the presence of a specific signal. Figure 2.9 shows the use of dissolvable hydrogels as selective sacrificial structures for microfluidics. Three types of hydrogels were photopolymerized inside a microchannel using sequential polymerization (Figure 2.9a). The two hydrogel walls blocking the entrances to the top and bottom branches have two different types of breakable crosslinkers, while the hydrogel post in the center was polymerized from hydrogel materials with non-breakable crosslinker. In the presence of one type of de-crosslinking reagent, the bottom hydrogel wall dissolved to selectively open the bottom branch (Figure 2.9b). The other hydrogel wall can be dissolved in the presence of a different de-crosslinking reagent, leaving both top and bottom branches open (Figure 2.9c). The hydrogel post in the center was not affected by either of the de-crosslinking reagents, suggesting that good selectivity can be achieved. This approach could further simply the system design toward autonomous microfluidic systems.
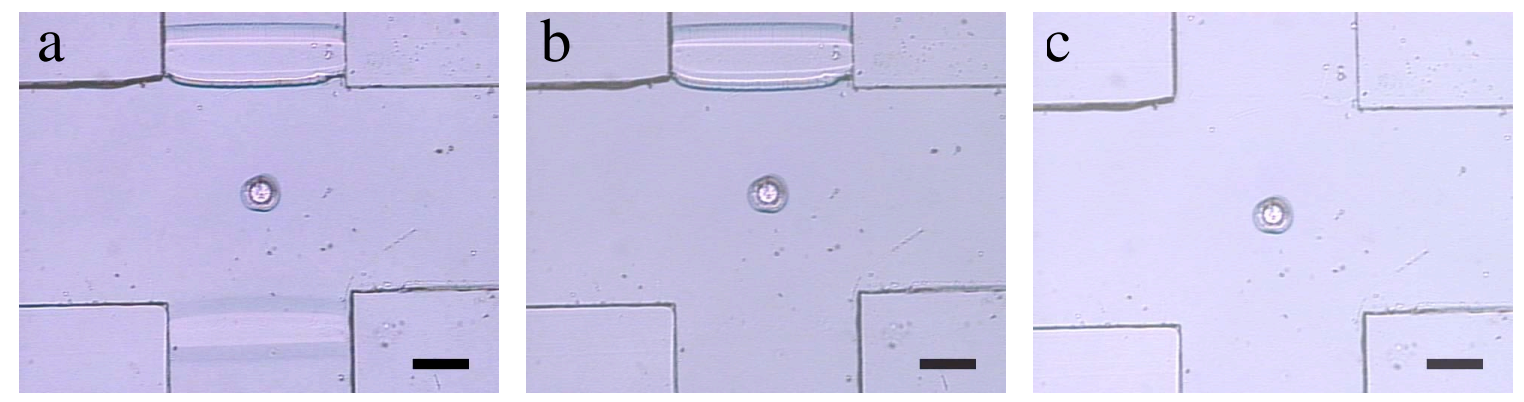

Figure 2.9. Two hydrogel walls were polymerized from mixtures with different breakable crosslinkers, while a hydrogel post was polymerized from a mixture with non-breakable crosslinker (a). One of the hydrogel walls dissolved completely in the presence of $5 \% \mathrm{NaIO}_{4}$ solution at a flow rate of $0.1 \mathrm{~mL} / \mathrm{min}$, while the other hydrogels were not affected $(b)$. The other hydrogel wall was dissolved using $10 \mathrm{mM}$ TCEP solution at a flow rate of $0.1 \mathrm{~mL} / \mathrm{min}(\mathrm{c})$. The hydrogel post in the center was not affected. Bars shown are $500 \mu \mathrm{m}$.

\section{Virtual Walls}

Although not envisioned in the original proposal, a method for building virtual walls within a microchannel was developed [9]. Manipulating gas and liquid fluids within networks of microchannels is crucial in the design and fabrication of microfluidic devices for applications in bioassays, microreactors, and chemical and biological sensing. We have used self-assembled monolayer chemistry in combination with multistream laminar flow to pattern surface free energies inside microchannels (Figure 2.10). These patterned channels demonstrate the principle of surface-directed liquid flow in which aqueous liquids are confined to the hydrophilic pathways, provided the pressure is maintained below a critical value. The maximum pressure is determined by the surface free energy of the liquid, the contact angle of the liquid on the hydrophobic regions, and the channel depth. On the basis of the maximum pressure differences 
for monolayers with different contact angles, simple pressure-sensitive switches were designed and fabricated inside channel networks to control liquid flow. A turn can be introduced into the
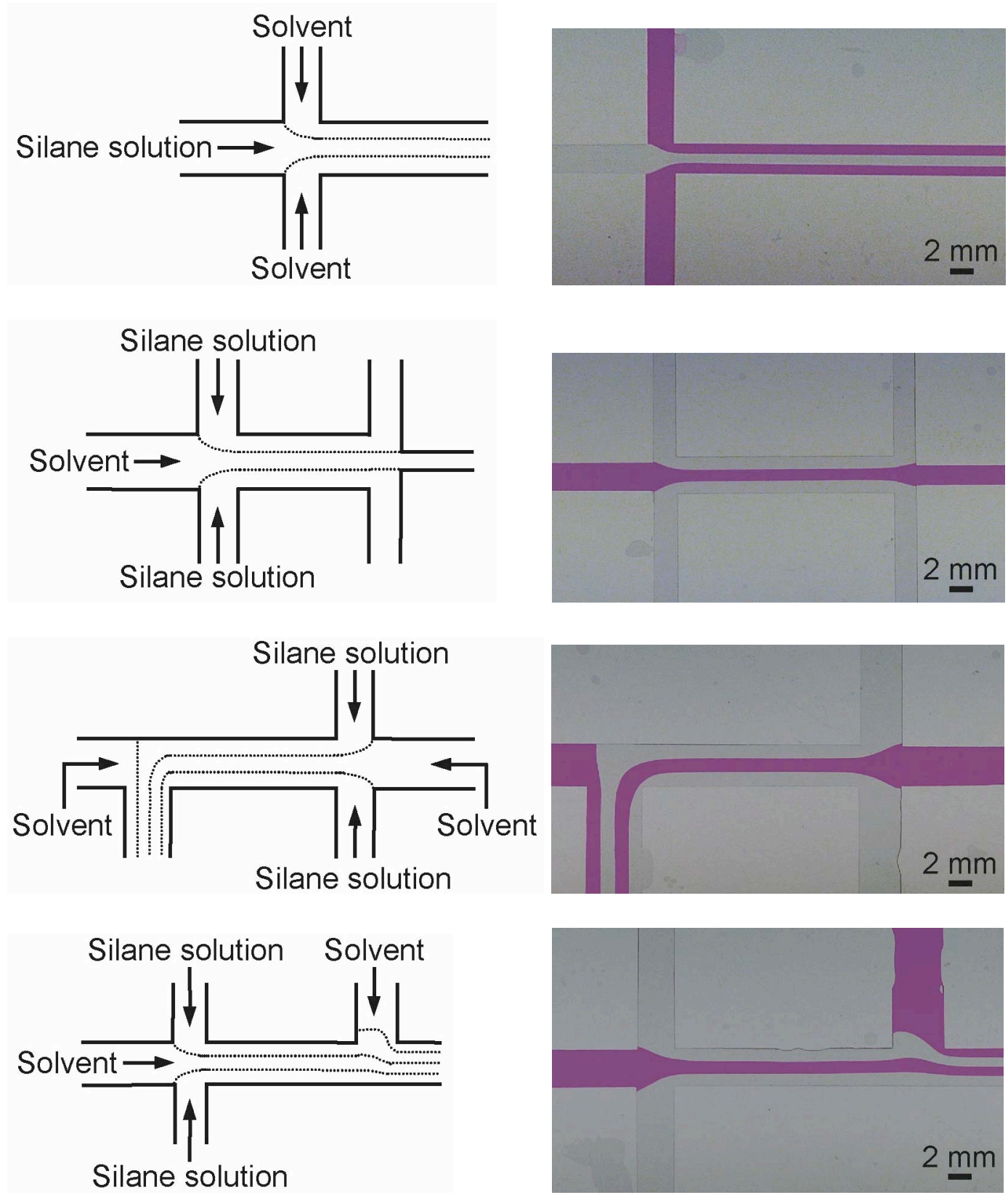

Figure 2.10. Schemes of multiphase liquid laminar flows and corresponding images of aqueous liquid flow inside channels after surface patterning. The liquid is a dilute solution of Rhodamine $B$ dye (conc. $0.057 \mathrm{w} / \mathrm{w} \%$ ) in deionized water. 
hydrophilic pathways to change the flow direction, and the critical radius of curvature is dependent on the contact angle and the channel depth. Photolithography was successfully developed to pattern surface free energies inside microchannels to overcome the limitations of multistream laminar flow fabrication. This method provides greater flexibility in the design and fabrication of complex flow patterns and facilitates mass manufacturing of surface-directed liquid flow devices. The ability to confine liquid flow inside microchannels with only two physical walls is expected to be useful in applications where a large gas-liquid interface is critical.

Liquids can be confined in hydrophilic regions, provided the pressure is below a critical value. The liquids are referred to as being confined by virtual walls. The underlying principles of surface-directed liquid flow have been investigated theoretically and verified experimentally. Theoretically, the maximum pressure $P_{\max }$ that virtual walls can withstand is $(2 \gamma / \mathrm{h}) \sin \left(\theta_{\mathrm{n}}-90^{\circ}\right)$, where $\gamma$ is the surface tension of the liquid, $h$ is the channel depth, and $\theta_{n}$ is the advancing contact angle of the liquid on the hydrophobic region. The critical width of the liquid stream $\mathrm{w}_{\mathrm{c}}$ under spontaneous flow conditions is $h /\left(\cos \theta_{p}\right)$, where $\theta_{p}$ is the contact angle of the liquid on hydrophilic region. The critical radius of curvature of a "turn" $R_{c}$ that liquid stream can make is $\mathrm{h} /\left[2 \sin \left(\theta_{\mathrm{n}}-90^{\circ}\right)\right]$. The minimal distance $\mathrm{d}_{\mathrm{m}}$ for two liquid streams to remain separated under the maximum pressure is $\mathrm{h}\left[1-\cos \left(\theta_{\mathrm{n}}-90^{\circ}\right)\right] / \sin \left(\theta_{\mathrm{n}}-90^{\circ}\right)$. Photocleavable self-assembled monolayers were synthesized and characterized before and after UV irradiation with contact angle goniometer and XPS. Experiments were designed to study the surface-directed liquid flow in various surface patterns within microfluidic channels. The results are liquid streams separated by $50 \mu \mathrm{m}$ under different pressures. The liquids bulged out under the higher pressure (Figure 2.11a) as indicated by thicker edges in the micrographs compared to the liquid streams under a lower pressure (Figure 2.11b).

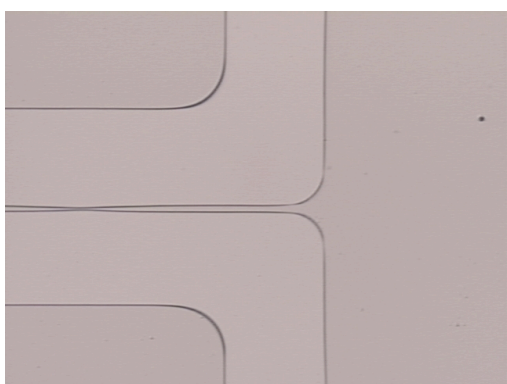

(a)

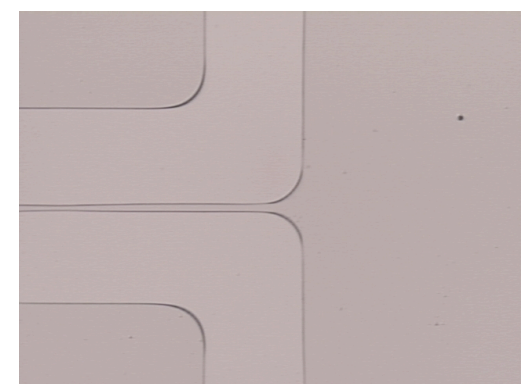

(b)

Figure 2.11. Water confined by a virtual wall under a) $12 \mathrm{~mm}$ of water pressure and b) $5 \mathrm{~mm}$ of water pressure.

A breakdown of "virtual walls" has been shown using steam. During an attempt to conduct a chemical reaction "across" a virtual wall via a reactive gas, it was observed that steam was generated from an exothermic reaction of nitric acid and water. The reagents were deposited on the hydrophobic channel, and the breakdown resulted in a relatively smooth collapse of the virtual wall (Figure 2.12). Such a phenomena could be used to selectively collapse one virtual 
wall while leaving others intact. Selective mixing of reagents could be accomplished using this method.

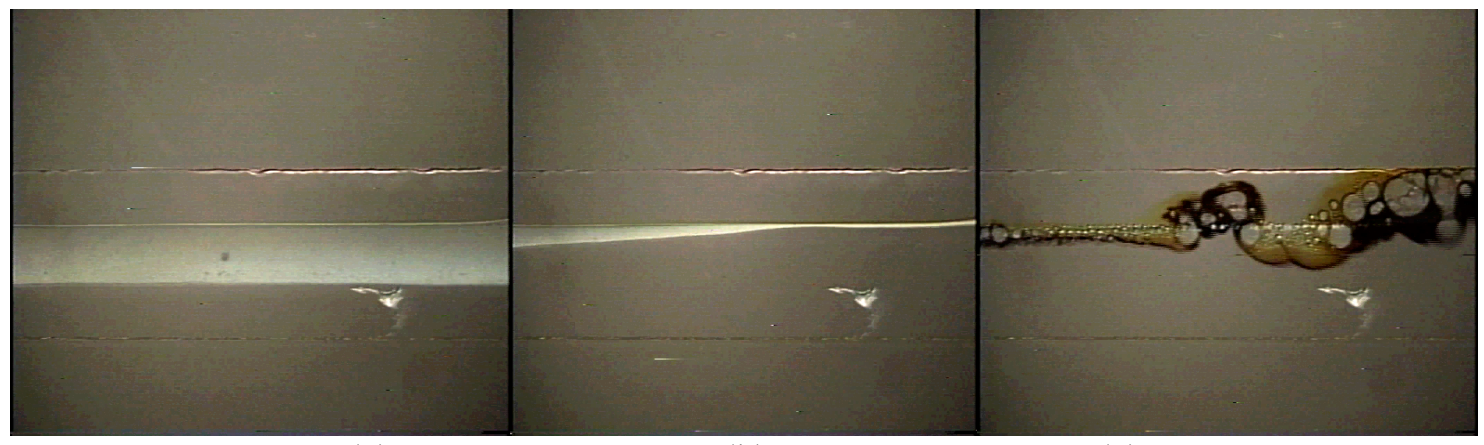

(a)

(b)

(c)

Figure 2.12. Solution on top is $1 \mathrm{MKI}$, on bottom is concentrated nitric acid. a) residual water in system mixes with nitric acid to create steam (whitish). b) wall starts to give out. c) redox reaction ensues.

\section{Active Assembly}

Active assembly methods for microfluidic systems are composed of several in situ functions that include blocking, delivering, control, etc. These functions are realized by using the spring force of a swollen hydrogel. Microfluidic systems often incorporate temporary or auxiliary channels to modulate the fluid flow. These channels are used for protecting certain sensitive components (i.e.; filter, readout post, etc.). The blocking function can be used for closing those channels or to detour the flow direction (Figure 2.13). Active assembly methods include other functions such as safety or release valves, degradable blocking components, etc. This method may allow additional tools to lower the fabrication limitation of microfluidic systems.

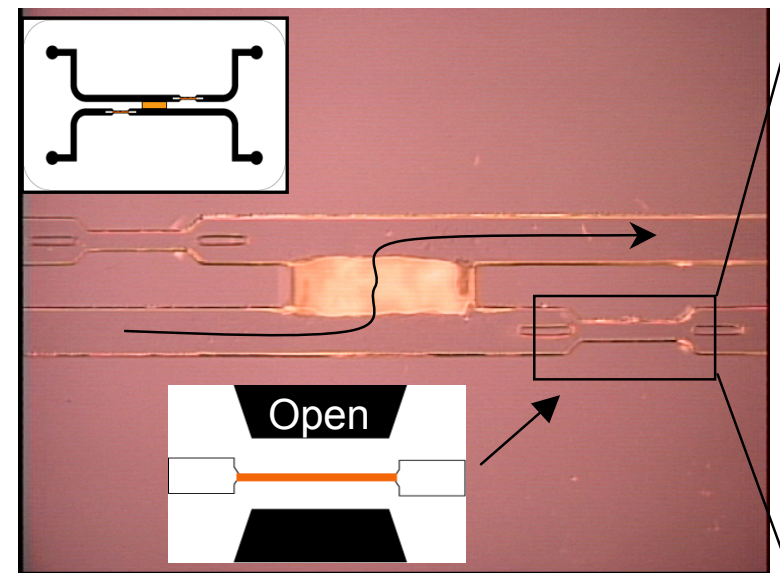

(a)

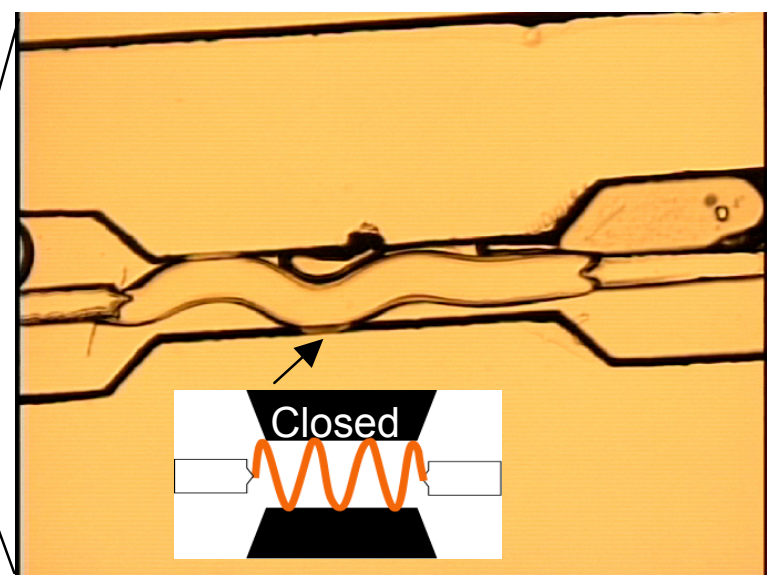

(b)

Figure 2.13. Schematic (inset) shows an example of the active assembly method applied to filter fabrication. The white area between the top and bottom channels in the picture $(a)$ is an in situ 
fabricated filter. The washing flow comes from left to right while not passing through the fabricated filter during the cleansing process. Once the filter is fabricated, the blocking components (b) located in the top left and bottom right side of (a) blocks the flow causing the sample solution to flow in the direction indicated by the curved arrow as shown in (a).

\section{Valves}

During the course of the project, several different types of valves were developed. The type of device dictated which valve was needed. A summary of the different valves is presented below.

\section{Bi-strip Valve}

A bi-strip hydrogel valve was fabricated [14] using a combination of sequential polymerization and parallel polymerization. The demonstration provides a good illustration of the potential range of devices made possible by $\mu$ Fluidic Tectonics. In a typical procedure, after polymerizing a pair of $\mathrm{pH}$-sensitive hydrogel strips simultaneously, the channel is flushed and filled with a non $\mathrm{pH}$-sensitive monomer mixture. A pair of non $\mathrm{pH}$-sensitive hydrogel strips is then polymerized at an adjacent location, slightly overlapping with the previous hydrogel strips. The non $\mathrm{pH}-$ sensitive strips also have anchors that fix one end of each bi-strip hydrogel to the channel at the desired location (Figure 2.14). When exposed to a basic solution, the pH-sensitive strip swells while the other strip remains the same volume, causing the bi-strip gel to extend and curve towards the non $\mathrm{pH}$-sensitive strip, thereby closing the channel (Figure 2.14c). In this way, it operates like the venous valves, allowing the fluid flow in only one direction. When contracted in acidic solution, the valve becomes deactivated and remains permanently open (Figure 2.14d).

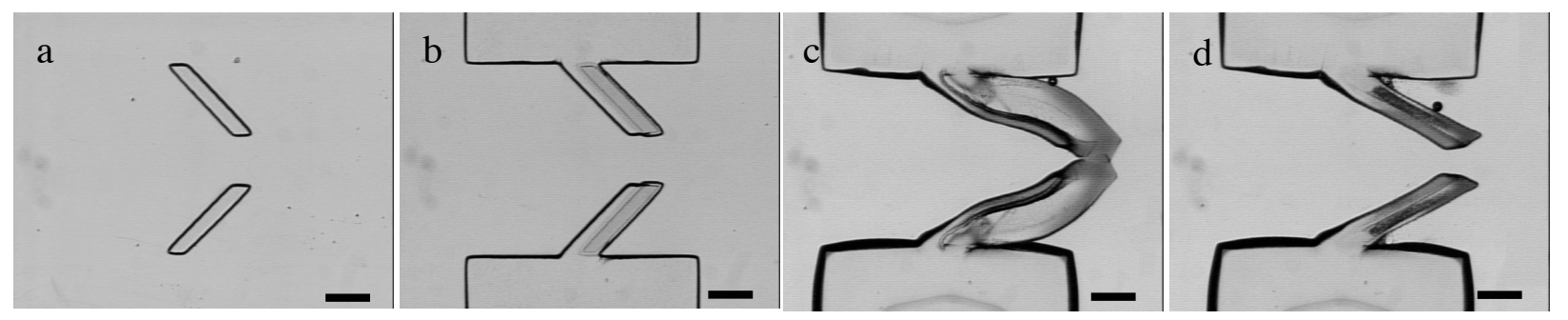

Figure 2.14. Fabrication of the biomimetic hydrogel check valve. A. After simultaneous polymerization of the $\mathrm{pH}$ sensitive hydrogel srips $b$. after polymerization of the non $\mathrm{pH}$ sensitive strips to for the bi=srip hydrogel valves with anchors. $C$. When exposed to basic solution, $k$ the bistrip hydrogel expands and curves to form a normally closed vlave. D. when exposed to ascidic solutions, the valve is deactiveate, returning to the permantly open state scale bars $500 \mu \mathrm{m}$.

Pressure measurements were carried out to characterize these hydrogel check valves. When activated in basic solutions, the $\mathrm{pH}$-sensitive strips serve as springs to provide a restoring force for the valves. Figure 2.15 shows a typical pressure profile upon forward fluid flow. After starting the flow at $1.0 \mathrm{~mL} / \mathrm{min}$ using a syringe pump, the pressure across the channel rapidly 
reaches $1.5 \mathrm{psi}$, pushing the check valve open to allow fluid flow. Upon stopping the flow, the valve gradually restores its closed position until the pressure gradient across the valve drops to zero. By adjusting the distance between the bi-strips and the degree of curvature of each bi-strip hydrogel, the pressure necessary to push open these check valves can be easily adjusted.

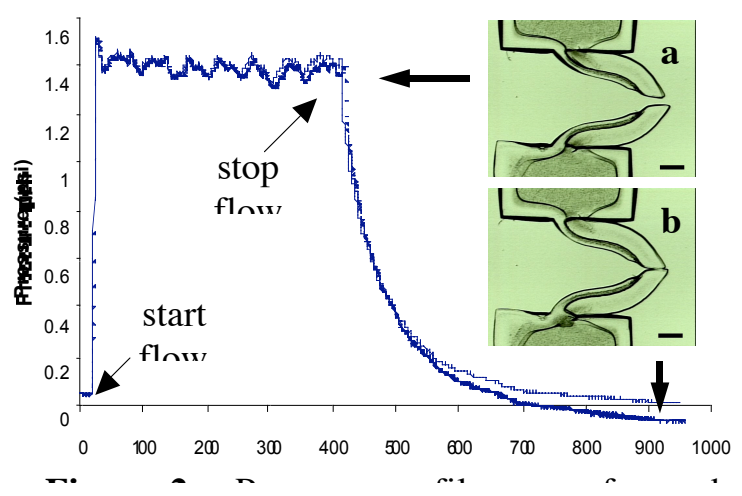

Figure 2.15. Pressure profile upon forward flow. After staring fluid flow at $1.0 \mathrm{~mL} / \mathrm{min}$, the pressure increases reapidly. When the pressure reaches 1.5 psi, the valve opens to allow forward fluid flow. Upon stopping the flow, the valve gradually closes.

A fully integrated in-channel fabrication of multiple microfluidic check valves has been developed. The process is shown in Figure 2.16. The valve anchors are formed separately from the channel walls to add the flexibility of placing the valves at any location. However, steps one and two in Figure 2.16 can be combined, leaving only the integration of the responsive hydrogel material to complete the valve.

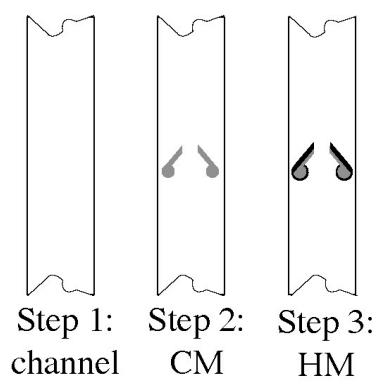

Figure 2.16. Formation of microchannel and valve. a) Step 1: formation of channel via URP process. b) Step 2: flow in construction material and polymerize valve anchor. c) Step 3: Flow in responsive hydrogel material and polymerize valve.

One immediate application of the check valves is to prevent backflow and contamination for multi-channel inlets of a miniaturized bioassay. Each inlet would contain a valve that would allow reagents to flow in the forward direction but not encounter each other until they have reached the center channel of a three-inlet device. An example of this is shown in Figure 2.17a and illustrates three separate colors flowing through a multichannel device. No backflow of the 
different colors can be seen due to the operation of the check valves. The human body utilizes redundant valves in the circulatory system, this principle is also applied here and shows the construction of such multiple valves in Figure 2.17b,c.

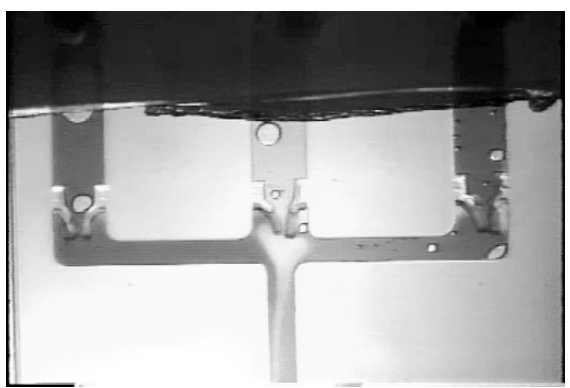

(a)

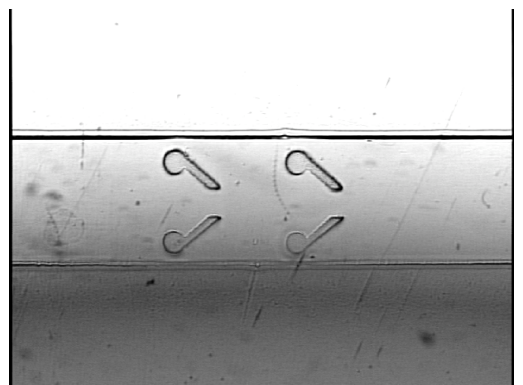

(b)

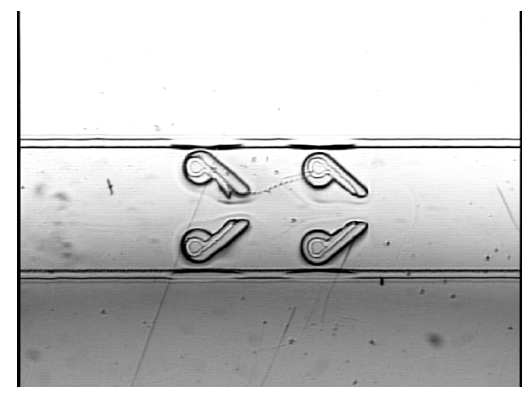

(c)

Figure 2.17. a) A multichannel device shown with different colored dyes and check valves that prevent backflow. b) The construction of multiple non-responsive valve anchors in a microchannel, and c) the addition of the responsive component of the valves is shown.

\section{Arrow Valve}

Another version of a tectonics microvalve utilized a different geometry. The micro check-valve was activated via hydrogel swelling and utilized the spring force of the swollen hydrogel. The valve was fabricated in situ in less than 10 minutes and is composed of two main parts. The first part is the anchor that includes a neck, guide and post as shown in Figure 2.18. The anchor is rigid and made of construction material, and the second part is the hydrogel piston comprising the head and body. The valve neck and head are designed to have similar contour lines to ensure a seal when they contact each other. The valve post supports the body and confines the swelling direction of the body to ensure swelling travels toward the neck. The hydrogel piston is directed through the guides toward the neck of the channel, and these guides prevent stray movements. The head connects with the neck of the channel while the body pushes the head with the spring force. The hydrogel recipe used for the valve is shown in Table 2.1. The recipe was optimized through experimentation to have proper swelling ratio, spring force, and adhesion to the channel surface and is shown below.

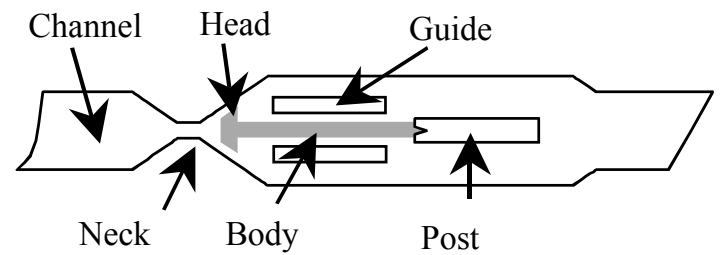

Figure 2.18. Schematic representation of the hydrogel valve 


\begin{tabular}{|c|c|}
\hline Material & Amount \\
\hline HEMA (monomer) & $3 \mathrm{~g}$ \\
\hline \hline EGDMA (crosslinker) & $0.09 \mathrm{~g}$ \\
\hline DMPA (photoinitiator) & $0.09 \mathrm{~g}$ \\
\hline
\end{tabular}

Table 2.1. Recipe for the hydrogel valve

Forward flow opens the valve by pushing the head and reverse flow seals the valve by increasing the pressure on the back of the valve head (Figure 2.19). The pressure curve measured to quantify the performance of the valve for forward flow is shown in Figure 2.20. The graph indicates the operating pressure is around 1.5 psi. Altering the gap between the neck and head or the width and length of the body can modify the operating pressure. Additional tests were performed to test the operation of the valve in aqueous solutions (see Table 2.2) commonly used an ELISA assay. The valve operated normally in these solutions.

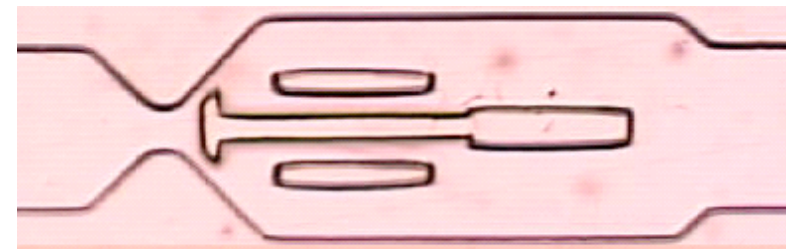

(a)

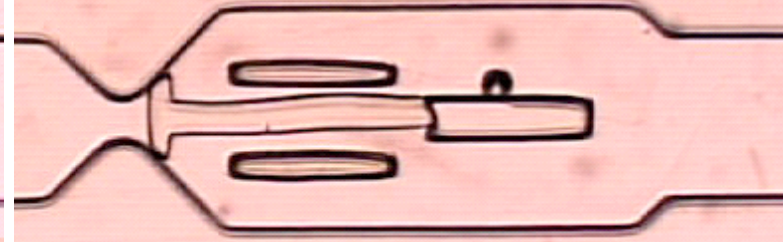

(b)

Figure 2.19. Hydrogel check-valve before (a) and after swelling (b).

\section{Pressure Curve}

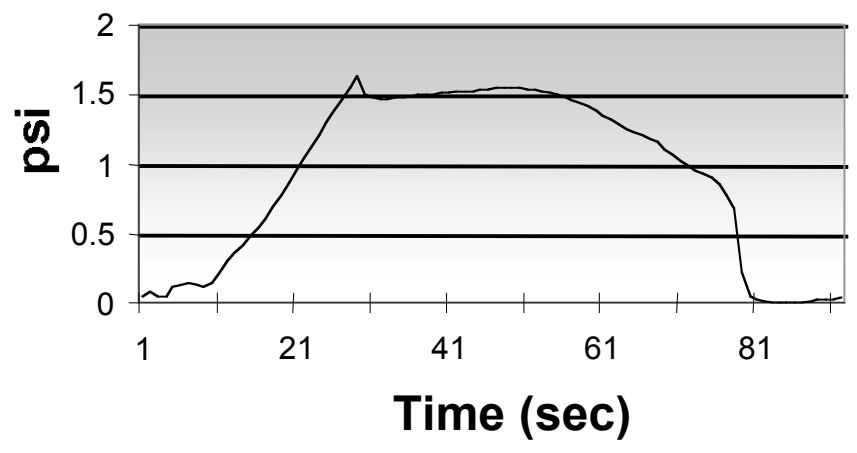

Figure 2.20. The operating pressure cureve of the hydrogel valve is shown for a forward flow rate of $0.5 \mathrm{ml} / \mathrm{min}$ 


\begin{tabular}{|c|l|c|}
\hline Solution & \multicolumn{1}{|c|}{ Composition } & pH \\
\hline Wash Buffer & TrisHCl 10mM, NaCl 150mM, Tween $200.005 \%$ & 7.4 \\
\hline Block Solution & Sodium Phosphate Monobasic $20 \mathrm{mM}, \mathrm{NaCl} 150 \mathrm{mM}$, Casein $1 \%$ & 7.4 \\
\hline Substrate & BCIP $400 \mathrm{mM}, \mathrm{NBT} 400 \mathrm{mM}$, Tris $100 \mathrm{mM}, \mathrm{MgCl}_{2} 5 \mathrm{mM}$ & 9.5 \\
\hline
\end{tabular}

Table 2.2. Tested solutions

\section{Filters}

The ability to filter solutions is vital in any bioassay. Therefore a means of filtering that can be adjusted for porosity and incorporated into microchannels is very important. Two types of filters both tectonics based and commercially available filters that can be incorporated into the channel device are presented.

\section{Tectonics Filter}

A tectonics filter is prepared by phase separation of a photo-polymerization of a mixture of monomer, porogen (e.g. water, salts), a cross-linker and a photo-initiator [15]. In this process, two immiscible phases are agitated to create droplets of one phase suspended in the second phase. Photo-polymerization of one phase results in the formation of polymer particles that subsequently join together. Upon further processing (e.g. drying to remove water), the porogen is removed to give a contiguous polymer particles network surrounded by interconnected channels. These channels were initially occupied by the porogen. The size and distribution of the pores and the mechanical properties are dependent on a number of factors including monomer and water concentration, crosslinkers, and initiator concentration. The large parameter space allows the filter property to be 'tuned' based upon the application. To see how the initiator concentration affects the porosity of the filter, the prepolymer mixture was prepared by varying the concentration of the initiator. The filter was then photopolymerized inside a glass capillary. Figure 2.21 shows the SEM image of the filters and shows that as the concentration of the initiator is increased, the pore size decreases initially, but above $1 \%$ concentration, the difference in porosity is minimal.

Filters with varying pre-filter compositions (Table 2.3) were characterized by confocal imaging and resistance measurements. Confocal microscopy gives an advantage over the traditional scanning electron microscopy techniques as the material can be imaged in the wet (or functional) state. To allow for visualization, the filter samples were coated with albumin-evan's blue complex. Upon flowing solution containing albumin-evan's blue complex through the filters, the albumin binds to the polyHEMA beads and labels them. The albumin-evan's blue complex is fluorescent with excitation around $490 \mathrm{~nm}$ and emission beyond $650 \mathrm{~nm}$. 


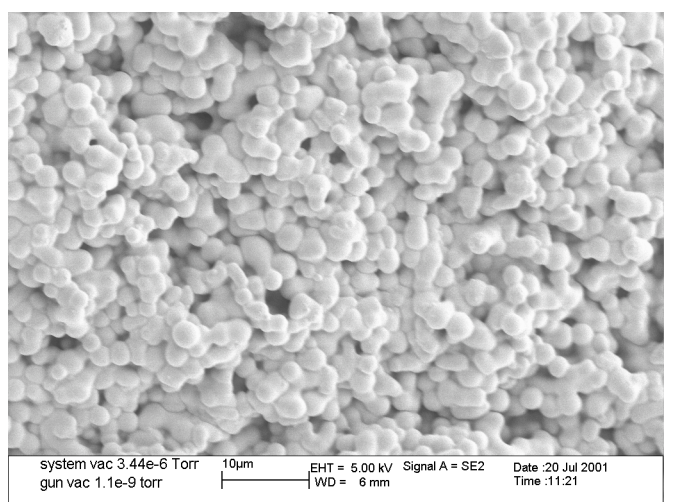

a

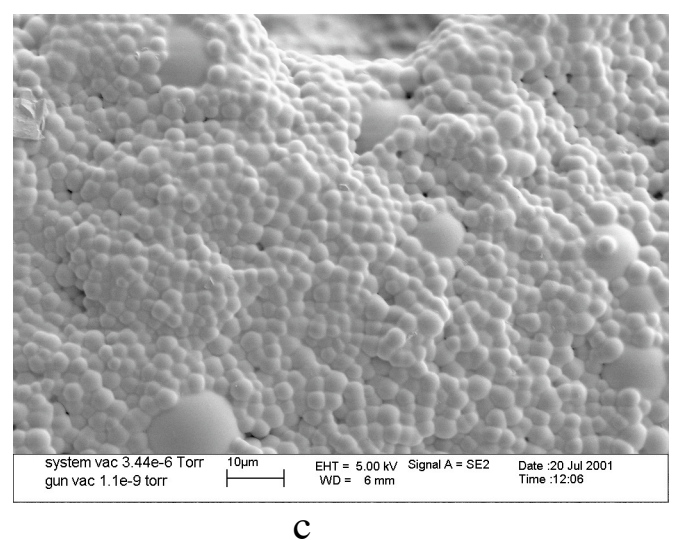

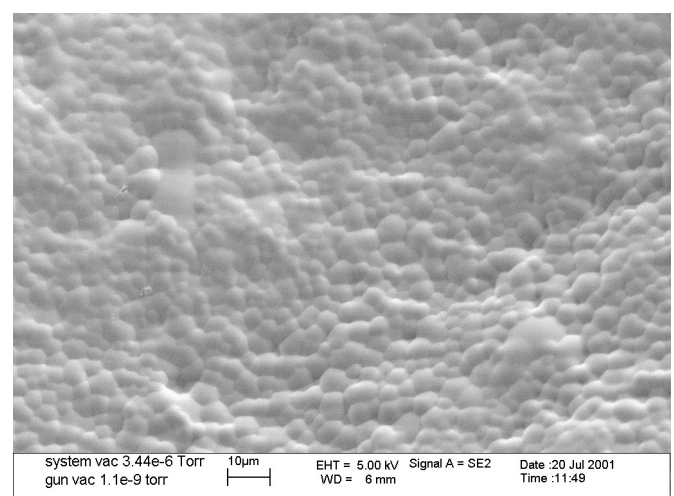

b

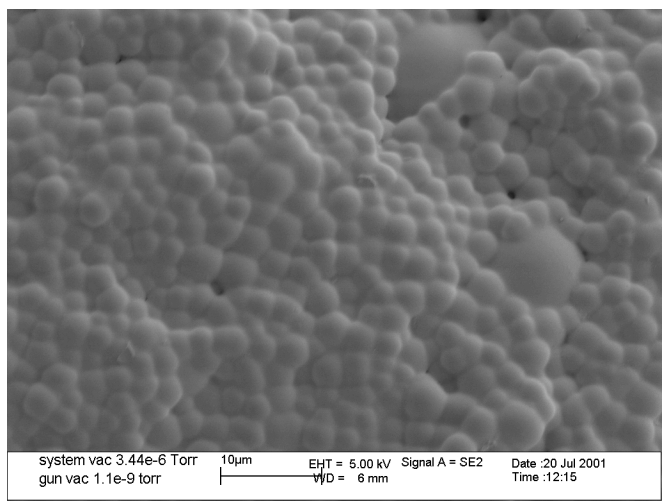

d

Figure 2.21. SEM images of a filter made with different initiator concentration. a) $0.378 \%$ b) $1 \%$ c) $1.5 \%$ d) $2 \%$ initiator. Scale bar is slightly different and is shown for each image.

The labeled filters were imaged with laser confocal microscopy (Bio-Rad MRC-1024). No washing step was required as the background interference was negligible. The size and distribution of the beads for the different filter types was thus visualized by confocal microscopy (Figure 2.22). The resistance across the filter was obtained by taking the ratio of the pressure (in psi units and measured using pressure transducer) and volume flow rate (in $\mathrm{mL} / \mathrm{s}$ and held constant). By dividing this ratio by the length of the filter (in $\mu \mathrm{m}$ ), the resistance for a micrometer length of the filter was calculated. Figure 2.23 shows the resistances for the different filter types. The resistance offered by F1 was the least while the highest was for the F2 filter.

\begin{tabular}{|r|r|r|r|r|}
\hline Type & $\begin{array}{c}\text { HEMA } \\
\% \mathrm{v} / \mathrm{v}\end{array}$ & $\begin{array}{c}\text { EGDMA } \\
* \% \mathrm{w} / \mathrm{w}\end{array}$ & $\begin{array}{c}\text { DMPA* } \\
\% \mathrm{w} / \mathrm{w}\end{array}$ & $\begin{array}{c}\text { Water } \\
\% \mathrm{v} / \mathrm{v}\end{array}$ \\
\hline F1 & 27 & 2.3 & 1 & 73 \\
\hline F2 & 27 & 3.3 & 1 & 73 \\
\hline F3 & 22 & 2.5 & 1 & 78 \\
\hline F4 & 22 & 3.5 & 1 & 78 \\
\hline
\end{tabular}

Table 2.3. Pre-filter compositions (* with respect to the weight of HEMA) 
The studies show that decrease in water concentration and increase in crosslinker concentration increases the resistance of the filter. Preliminary studies with rabbit whole blood cells demonstrated that the filter could be utilized to separate blood cells from proteins and other small molecules. Also, the large surface area of the porous filter can be used to carry out detection assays as the protein (albumin) preferably binds to the polyHEMA beads. Thus, with one component, separation, and subsequent detection assay can be carried out in a small region of the microsystem.

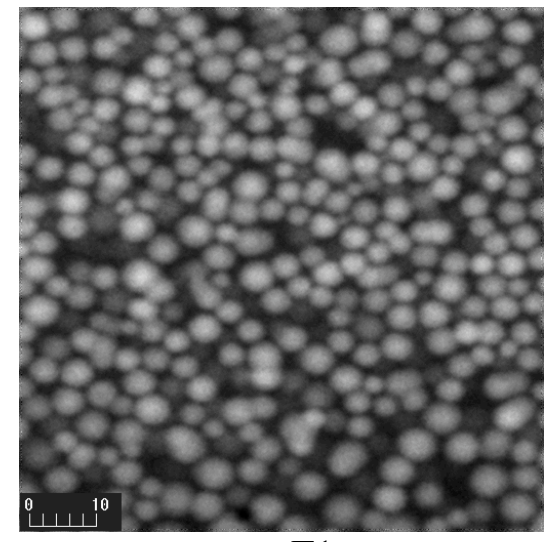

F1

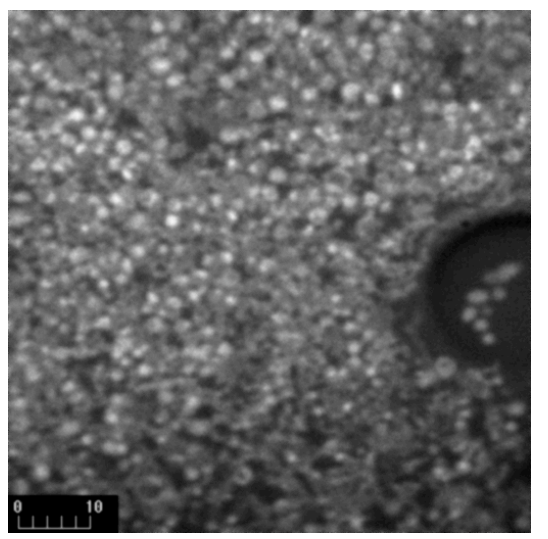

F4

Figure 2.22. Two examples of Confocal micrographs showing the pore size and distribution of the polyHEMA beads in the filter. The scale is shown within the images.

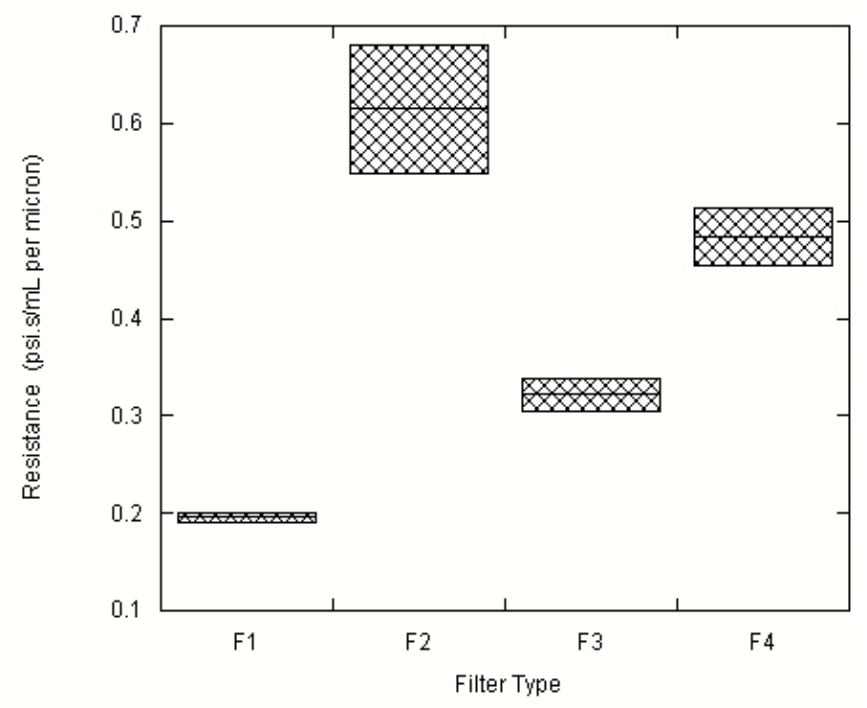

Figure 2.23. Resistance of the filters in a microchannel.

The utilization of the fabricated filter to perform biological separation was explored by separating blood cells from whole blood. Separation of blood cells from whole blood is required 
if the diagnostic device is to assay the body fluid directly from the patient (or end user). The present methodology is to use centrifuge methods. However, this process is difficult for very small sample volumes $(\mathrm{nL}-$ few $\mu \mathrm{L})$. Moreover, centrifuge requires external power for operation and may not be a practical in a portable diagnostic device. The average diameter of the rabbit red blood cells is about $7 \mu \mathrm{m}$. F1 and F2 filter types (Figure 2.24) were efficient in separating the blood cells from the whole blood. Upon flowing the whole blood past the filter, blood cells accumulated in and against the filter (Figure 2.25). The filtrate was collected and Glucose-6-phosphate dehydrogenase (G-6-PDH) assay was performed. The assay estimates the concentration of the enzyme, G-6-PDH. As the enzyme is present only inside the cells, the presence of G-6-PDH will indicate if cells were present in the filtrate or if the cells were lysed inside the filter. The results were compared with the unfiltered whole blood and with the supernatant after centrifuge. The enzyme kinetics was estimated from the absorption intensity at $340 \mathrm{~nm}$. Figure 2.26 shows the change in intensity over time. The comparison demonstrates that the filter is as efficient as centrifuge method in separating the blood cells.

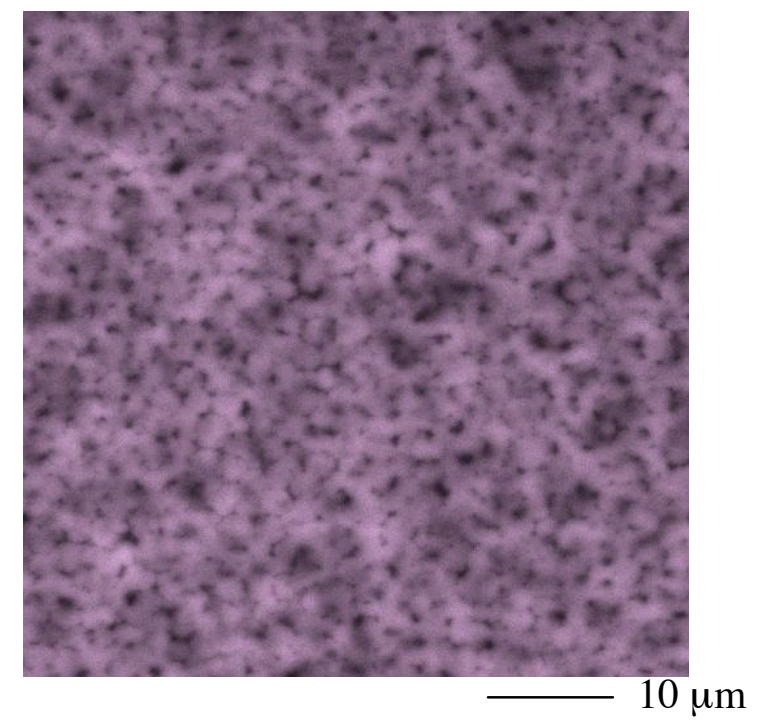

F1

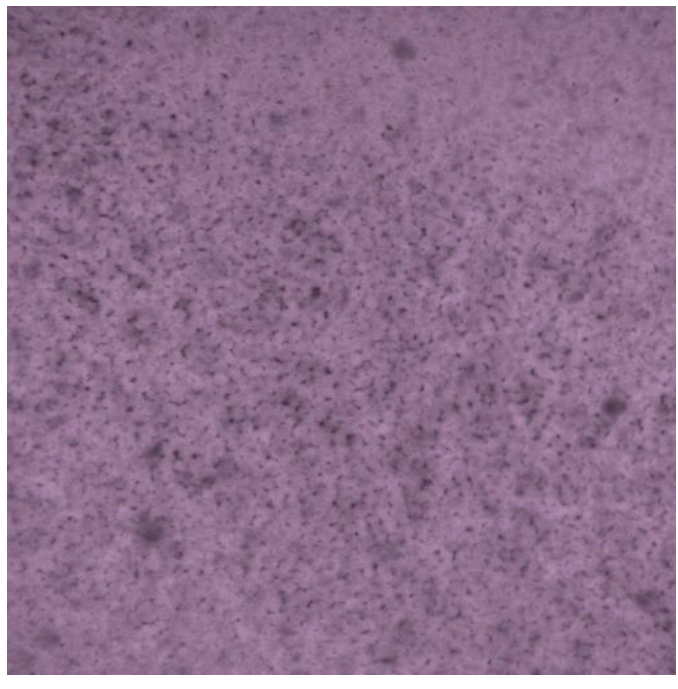

F2

Figure 2.24. Confocal micrographs of the 2 filters studied. F1 was prepared from $27 \%$ monomer, $2.3 \%$ cross-linker and $1 \%$ photo-initiator. F2 was prepared $27 \%$ monomer, $3.3 \%$ cross-linker and $1 \%$ photo-initiator 


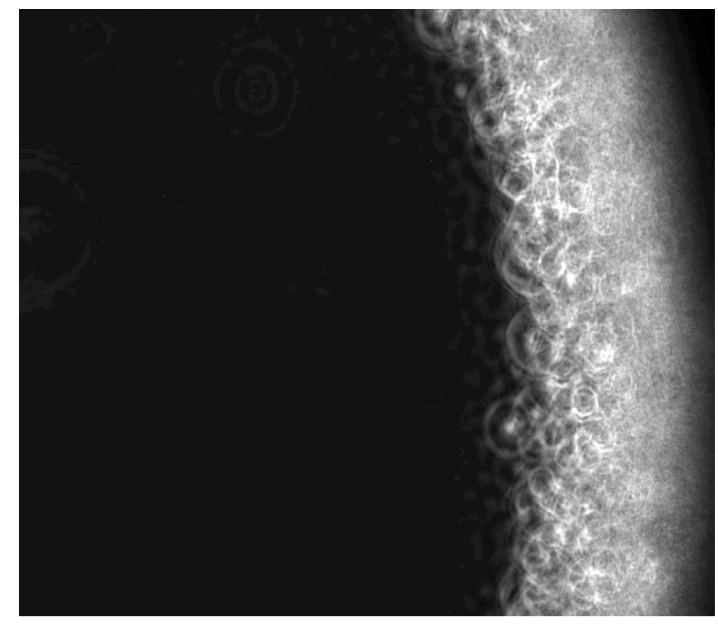

a

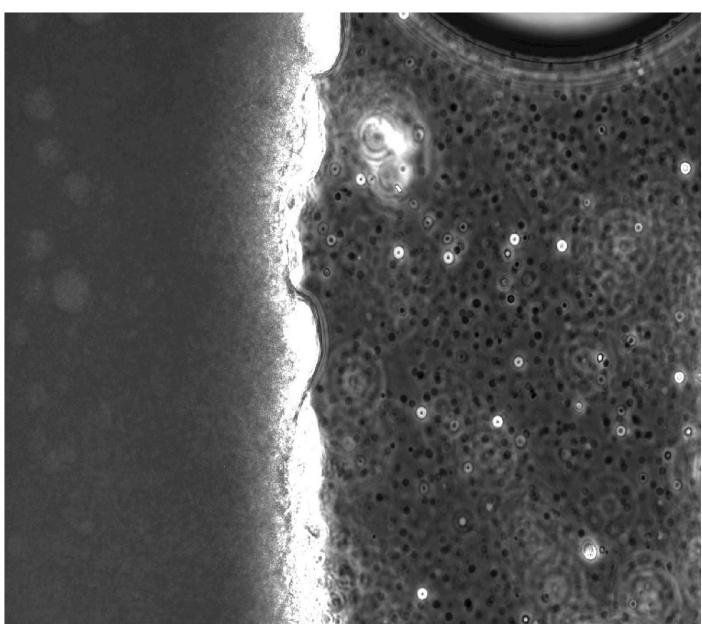

$\mathrm{b}$

Figure 2.25. Separation of blood cells from rabbit whole blood. (a) After filter (b) Before filter. The black dots are the red blood cells while the white dots are white blood cells. The cell size is around 6 um in diameter.

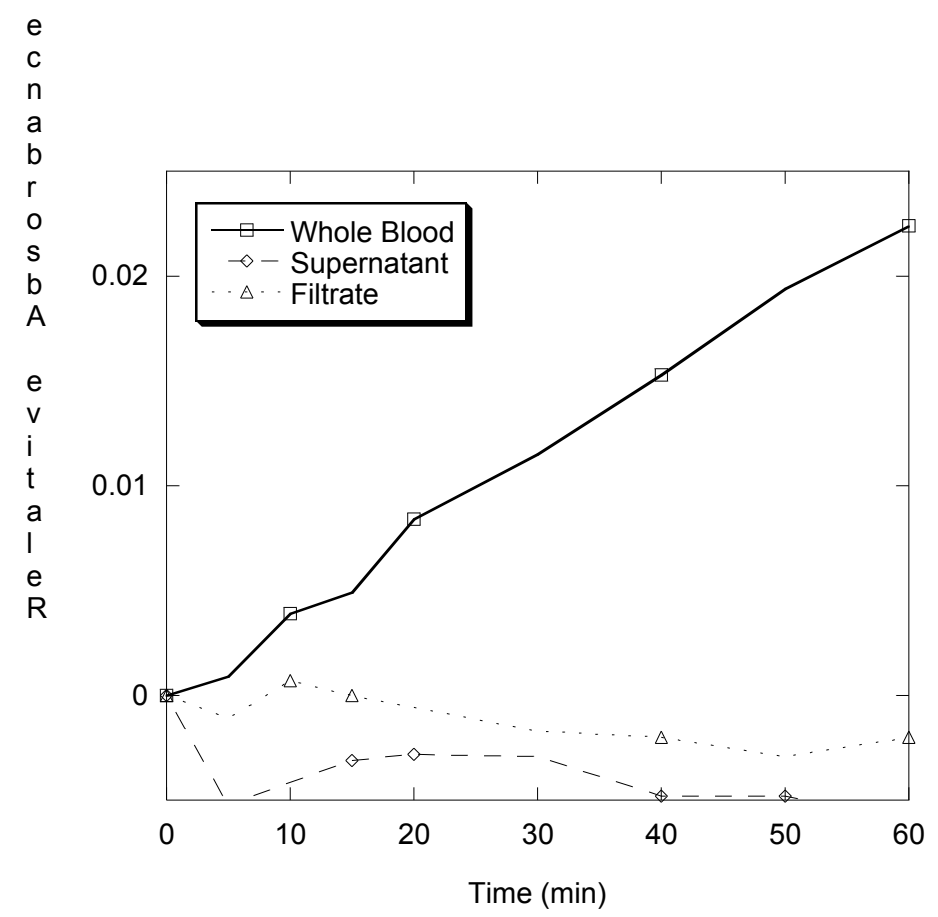

Figure 2.26. Kinetics of glucose-6-phosphate dehydrogenase enzyme. 


\section{Integrated Filter}

An alternative filter design is comprised of a commercially available polycarbonate screen filter that has through holes instead of a torturous path [13]. Using the layering technique, a device was formed with a hole cut into the top. The channel was filled with an immiscible fluid, and the filter was placed on top. A second layer was added, and the filter was polymerized in place during the formation of the channel (Figure 2.27). The filter pores do not appear clogged from the prepolymer solution during fabrication of the device as the channel was easily flushed. A high flow rate $(10 \mathrm{ml} / \mathrm{min}$ ) was used to introduce two types of beads ( 3 and 45 micron) into the filter. The filter allowed the 3 micron beads through initially and held the 45 micron beads on top. Due to the high concentration of the smaller beads, the filter began to get clogged but was still able to allow water and some smaller beads through (Figure 2.28).

The filter can be purchased with the correct pore size based on the desired filtering needs. For filtering blood, a 4.7 micron pore sized can be used and for any other filtering needs, the pore size can range from 0.01 to 20 microns. This filter can also be used to trap particles of interest in the microchannel and serve as a holding area to be observed under a microscope. This filter serves as an alternative to the tectonics filter and for added functionality of the $\mu$ FT platform.

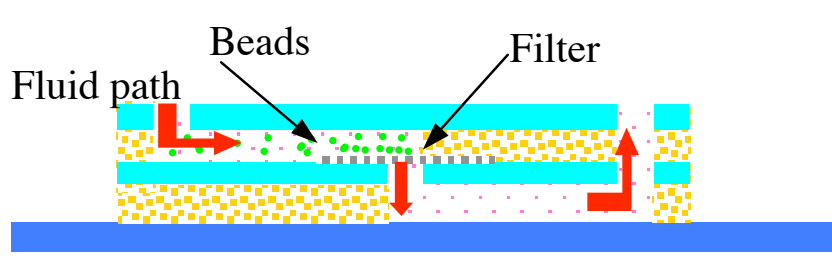

Side view

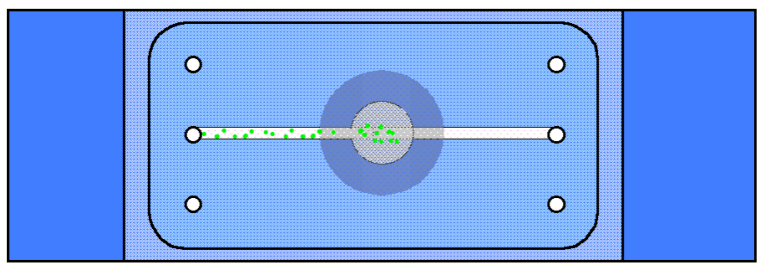

Top view

Figure 2.27. A top view and a side view of a two-layer device used to place the filter inline with the fluid path. Two different size beads ( 3 micron and 45 micron) were introduced into the device using a 5 micron pore size filter.

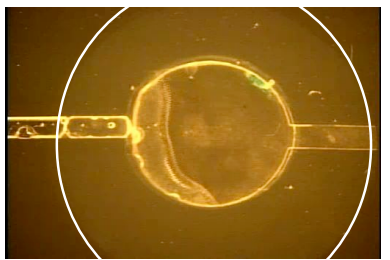

(a)

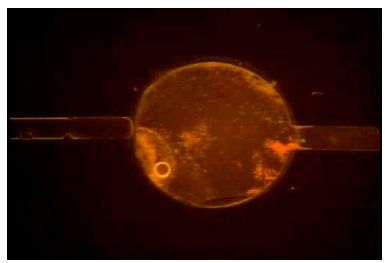

(b)

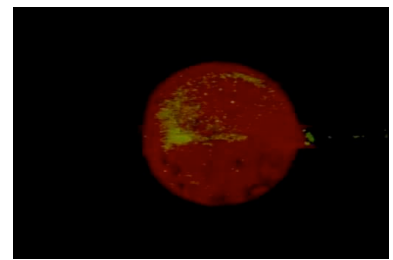

(c)

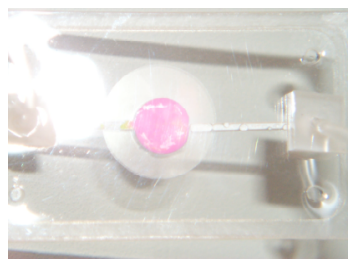

(d)

Figure 2.28. A closeup view (white circle indicates the outer filter edge) of the device is shown with beads flowing through (a) initially (b) with darkfield on and (c) after a few seconds at a flowrate of $10 \mathrm{ml} / \mathrm{min}$. A photograph (d) of the device is shown which shows the 3 micron beads (pink) in the filter and the shadow outline of the entire filter. 


\section{Sensors}

In a microchannel, the ability to monitor and read the conditions of an assay or test under investigation is necessary. A sensor or readout is used to give feedback of the conditions inside the channel with unaided human senses, and the simplest way to have a macroscale readout is with color change. Here we present several types of sensors that were fabricated inside the microdevices.

\section{$\mathrm{pH}$ Sensors}

A readout was prepared by dispersing $\mathrm{pH}$ sensitive dyes in a hydrogel matrix and then photopolymerizing the prepolymer mixture inside a microchannel [16]. The principle is similar to immobilizing dye into a matrix for preparing $\mathrm{pH}$ paper. However, the advantage of using the gel is flexibility in designing the device and also in geometry of the readout. We have tested two dyes - congo red (CR) and phenolphthalein (PP). CR changes color from blue to red at around 3 $-5 \mathrm{pH}$ while $\mathrm{PP}$ becomes pink from colorless at around $8-9 \mathrm{pH}$.

The time response of the gels is shown in Table 2.4. The color changes quicker at the higher $\mathrm{pH}$ since the gel swells and allows more solution to reach the immobilized dye. The CR responds slower than PP, and the exact cause for this difference is not yet clear. The response time for different flow rates $(0.01,0.05 .0 .1 \mathrm{~mL} / \mathrm{min})$ was tested and was found to be similar.

\begin{tabular}{|c|c|c|}
\hline & Congo Red (s) & Phenolphthalein (s) \\
\hline Dry-acid & 150 & - \\
\hline Acid-base & 45 & 20 \\
\hline
\end{tabular}

Table 2.4 Response time of the dye to acid and base

One drawback is that the dye leaks out of the gel once swelling and a color change has occurred. We tested the effect of flow rates on the recyclability for the readouts (Figure 2.29). The readouts were cycled between acid $(\mathrm{pH} 2)$ and base ( $\mathrm{pH} 12.5)$ solutions. The volume of solution was kept a constant $(1.38 \mathrm{~mL})$ hence the time in the solution varied for the different flow rates $(0.1,0.25,0.5 \mathrm{~mL} / \mathrm{min})$. Images were taken at appropriate time and the intensity value of the readout was extracted from the image using Adobe Photoshop 6.0. The intensity was normalized so that 1 in the graph indicates background color i.e. the gel is colorless. The trend indicates that flow rate is not the main variable that influences the response of the readout. Most likely, the main parameter is the exposure time in the solutions. The gels in the slow flow rate experiments were in contact with the solutions for longer duration which decreases the recyclability, while in the case of the fast flow rate $(0.5 \mathrm{~mL} / \mathrm{min})$ experiment, the gel was in contact with the solution for lesser time in each cycle and hence could withstand more cycles. Also another factor that 
increased the dye retention for the slow flow rate $(0.1 \mathrm{~mL} / \mathrm{min})$ is that upon swelling, the gels touch each other which makes the dye leak. For this reason, appropriate dimensions while designing the readouts is necessary.

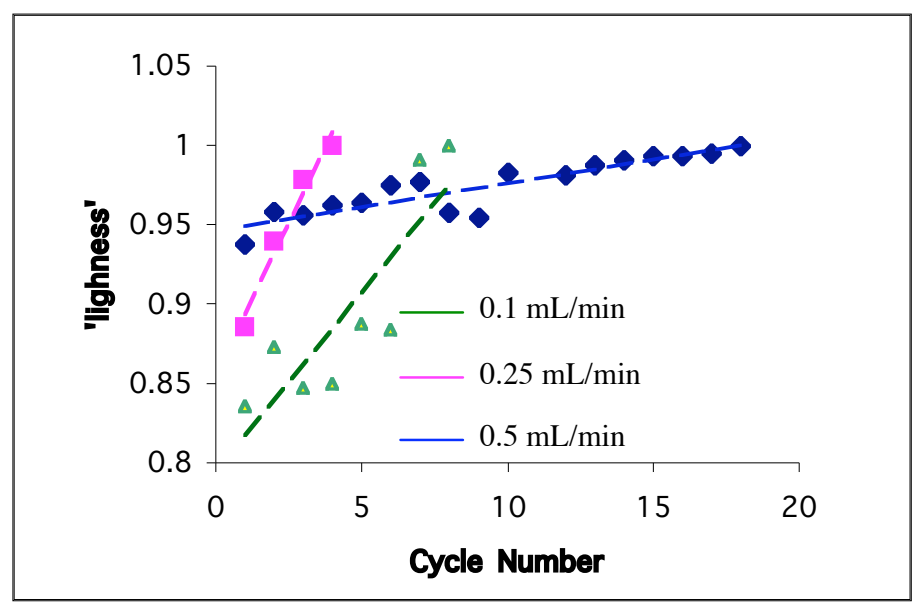

Figure 2.29. The decrease in intensity of the PP-gel for different flow rates.

A combinatorial readout can be prepared by polymerizing prepolymer containing different dyes. This combinatorial approach was demonstrated for CR and PP gels as shown in Figure 2.30.

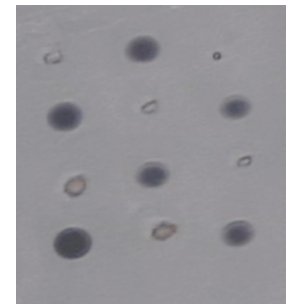

a

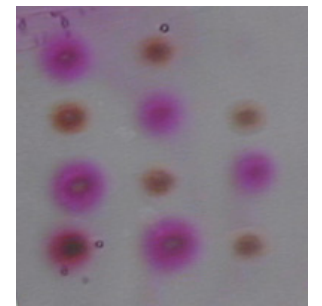

b

Figure 2.30. Combinatorial readout. a) at $2 \mathrm{pH}$ b) at $12 \mathrm{pH}$.

\section{Biosensors}

There are two approaches to making a gel into a biosensor; a passive approach that relies on colorimetric visualization, and the active approach that incorporates a biological binding event within the crosslinking of the hydrogel that leads to a volume change in direct response to a specific biological agent. Both approaches were investigated. 
By controlling the crosslinking of a gel, one can control the amount of protein intake of the gel. Likewise, by swelling the gel, one can control the diffusion of protein out of the gel. A model diffusion study is being conducted with bovine serum albumin in a $\mathrm{pH}$ responsive hydrogel (Figure 2.31). Understanding the diffusion mechanism, swelling properties, and protein interaction and retention of these materials may lead to new biosensing capabilities.

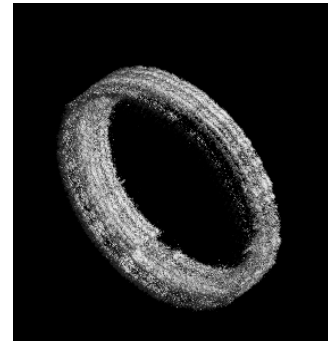

(a)

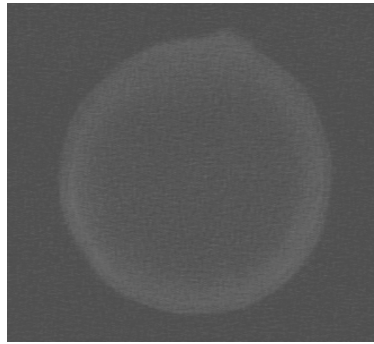

(b)

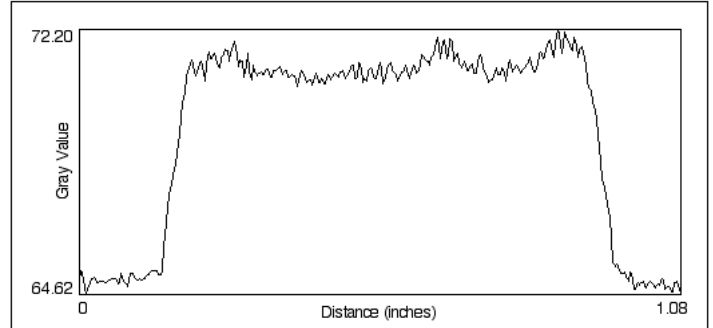

(c)

Figure 2.31. A confocal study of diffused protein (FITC-BSA) ring(a) around hydrogel. A micrograph of a $\mathrm{pH}$ responsive hydrogel $(b)$ with protein molecules embedded in the hydrogel matrix. Gray-scale intensity profile (c) of fluorescent protein content in a hydrogel.

A simple method of evaluating hydrogels as biological sensors was investigated with visual readouts. One critical question is the diffusion of the bioagent into the gel. In a model study, BSA was used as a model for a bioagent. A channel was filled with hydrogel posts (Figure 2.32a), and the posts were exposed to 5\% BSA in a phosphate buffer (Figure 2.32b) and show a color change due to BSA diffusing into the posts. Retention of the colored product was assessed with the introduction of other biological agents.

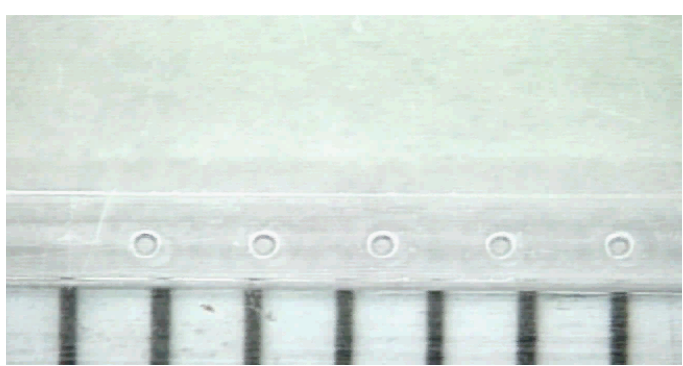

(a)

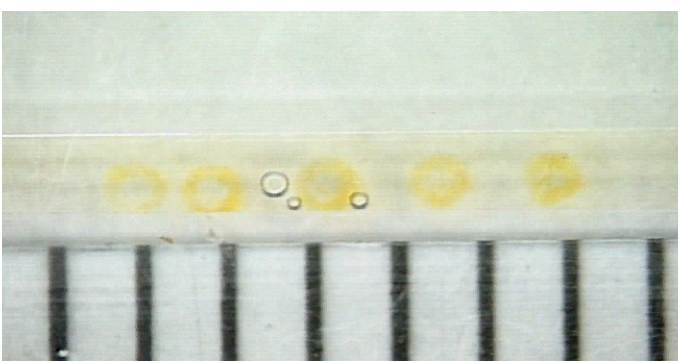

(b)

Figure 2.32. A channel (a) showing hydrogel posts before exposure and hydrogel posts with $5 \%$ BSA (b) in $0.05 \mathrm{M}$ phosphate buffer.

It was found that biological materials could be contained within the hydrogels, dried, stored, and still retain functionality. While signal is discernable around the edges of the gel, the top of the gel does not change color because it comes in contact with the top of the channel (Figure 2.33). Two methods were utilized to increase signal in the most visible area, the top of the gel, by increasing its exposure to solutions. The first method involved forming posts in smaller cavities, removing the tops, and forming a larger channel around the post (Figure 2.34a). The second 
method involved laminar flow of an inert solution on top of the hydrogel material, the post is polymerized inside the channel at a height smaller than the channel height (Figure 2.34b).

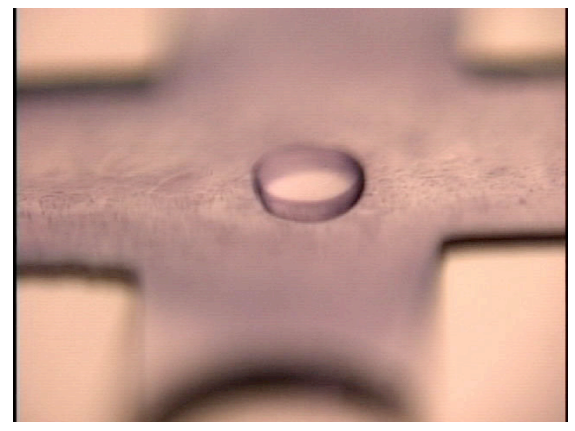

Figure 2.33. A sideview of an acryl amide gel containing biotinylated IgG, after exposure to Extravidin/Alkaline phosphatase conjugate and p-nitrophenyl phosphate.

Both methods were effective in controlling the initial gel size. The gels continued to swell to the tops of the channels, and modifications were made to the chemical formula to control the amount of swelling (Figure 2.35). Test gels were formed using an acryl amide monomer solution containing an increased cross-linker concentration. These gels, together with control gels, were dried at ambient temperature and later exposed to buffer solution. By coupling the modified construction methods with the increased cross-linker concentration, a readout structure with a robust signal was achieved (Figure 2.36 and 2.37).
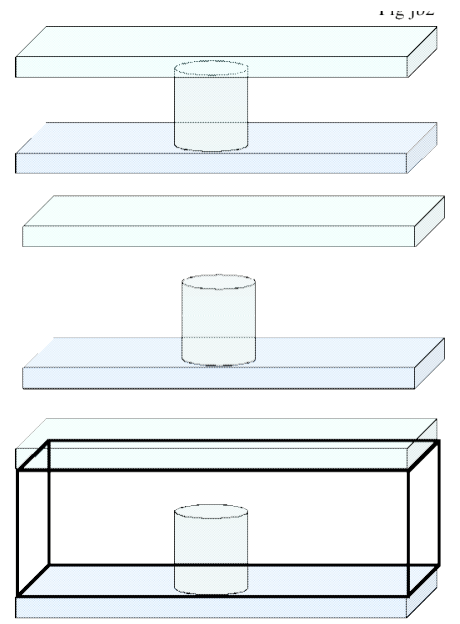

(a)
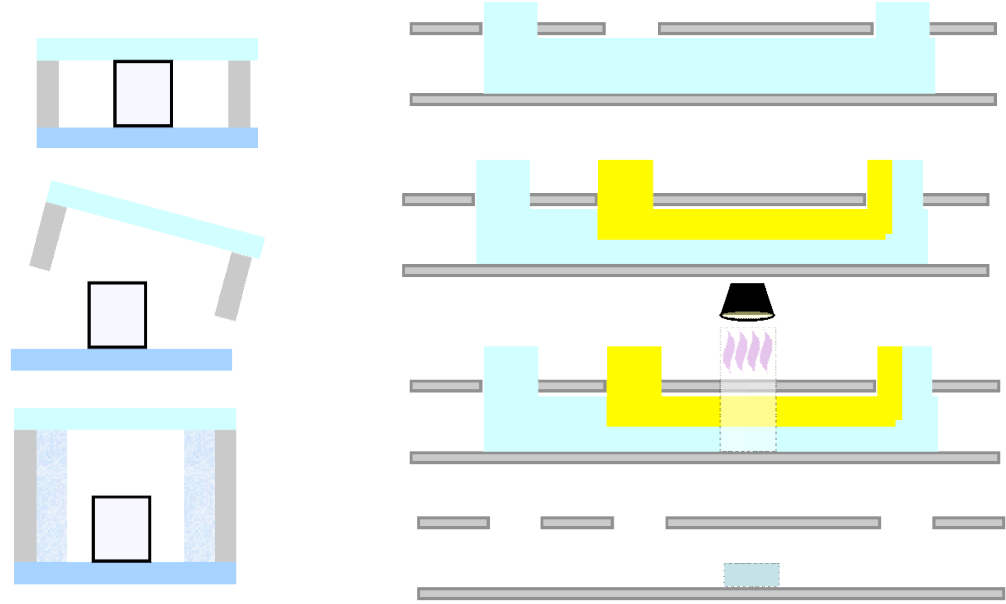

(b)

Figure 2.34. (a) Hydrogel posts are formed using a polycarbonate top with a gasket height of $125 \mu \mathrm{m}$. The polycarbonate top and gasket are removed, and a new polycarbonate top with a gasket height of 250 $\mathrm{\mu m}$ is put in place. Channels are formed around the gels. (b) Two inlet holes and one outlet hole are punched in the polycarbonate top and channels are formed. The 
hydrogel monomer is flowed in through first inlet well, and water is flowed in through the second inlet well, displacing the acryl amide monomer solution. UV light is used to polymerize the gel, and the channel is flushed, leaving a short gel on the bottom of the channel

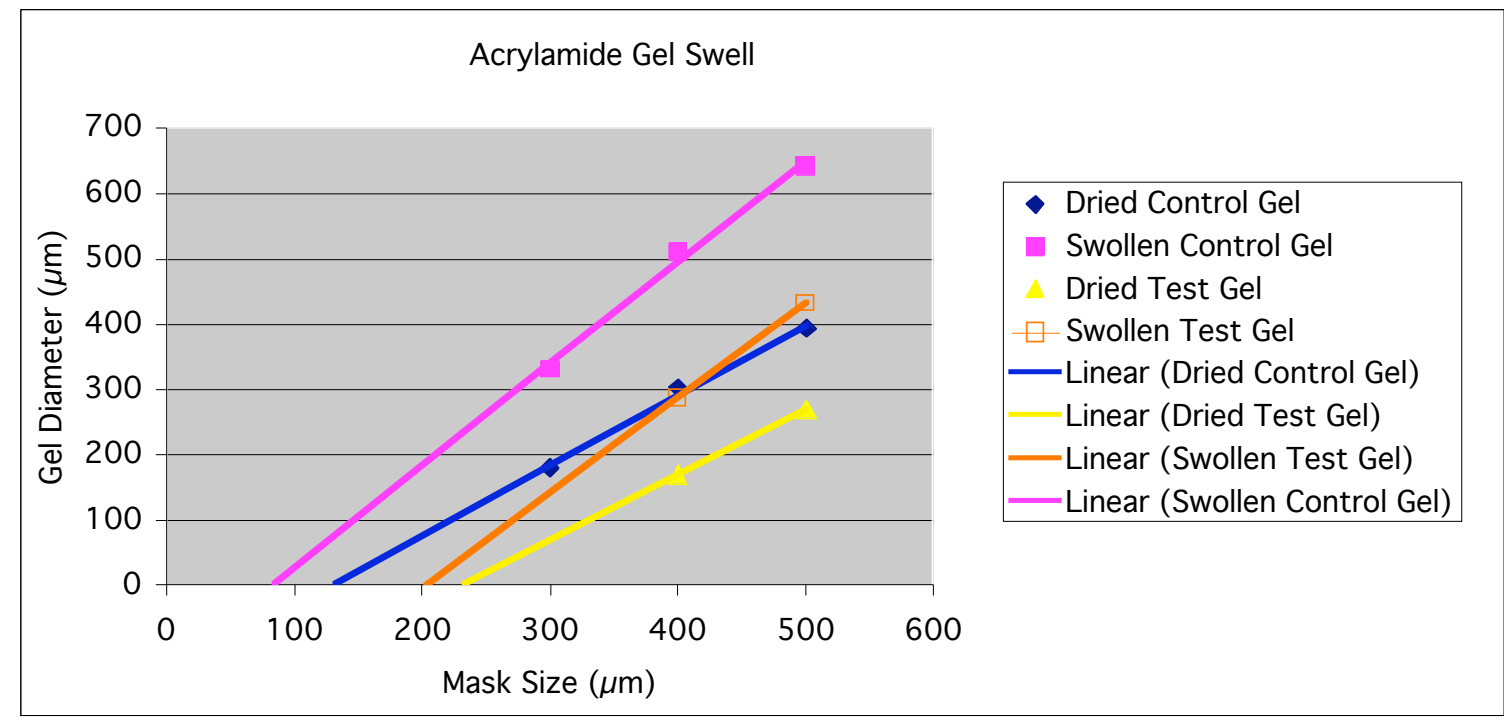

Figure 2.35. The diameters of all of gels with different amounts of crosslinker were measured. Results indicate that gel-swelling can be controlled by increasing the cross-linker concentration.

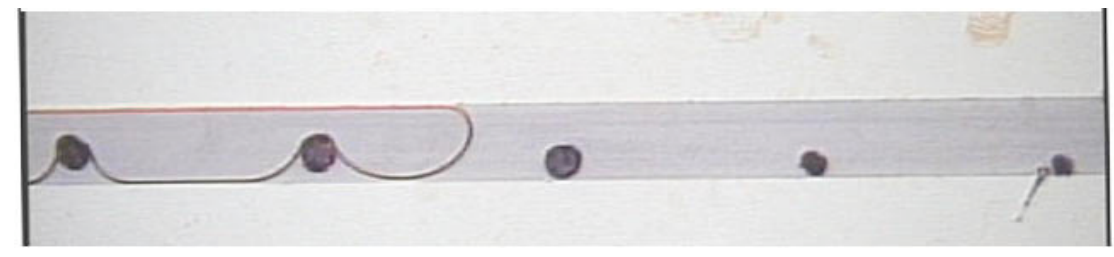

Figure 2.36. A top view shows $\sim 20 \mu \mathrm{m}$ tall reactive posts patterned by polymerizing Avidin in acryl amide gels. Channels were formed around the posts to direct reagent flow. An ELISA was performed, using biotinylated rabbit-anti-botulinum E as primary antibody, and ultimately produced a visible signal.

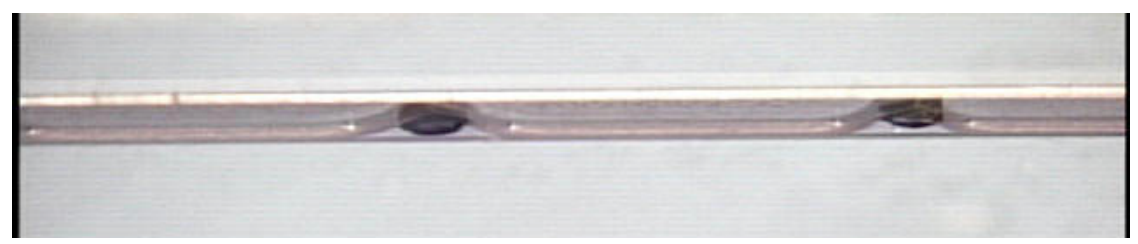

Figure 2.37. Side view shows the top of the gel does not impinge upon the top of channel. This provides maximum exposure of the gel to reagents, increased surface area, and intensification of signal to the observer. 
Another method of introducing biosensors into the devices was by using commercially available streptavidin-modified agarose gel (Sigma-Aldrich). The solution consisted of polymer beads that are 40-100 microns in diameter, and widely used in affinity chromatography. The presence of streptavidin allowed for the immobilization of biotin-conjugated antibodies. To test the solution, a 2-level channel was fabricated with a filter membrane in between the layers. The filter membrane was incorporated to hold the beads. To minimize the background, the channel and the filter were coated with casein prior to flowing in the bead solution. After the solution was introduced into the channel, the beads were incubated in biotin-conjugated antibody for (BoTX) for an hour. A sandwich ELISA was then performed (antibody-antigen-antibody) by flowing in protein solutions in the appropriate order. The incubation time was maintained at one hour. In parallel a control test was performed in which the addition of the antigen ( $\alpha$ toxoid) was omitted. Five minutes after introducing the substrate, the signal from the gel showed a higher intensity than the control (Figure 2.38).

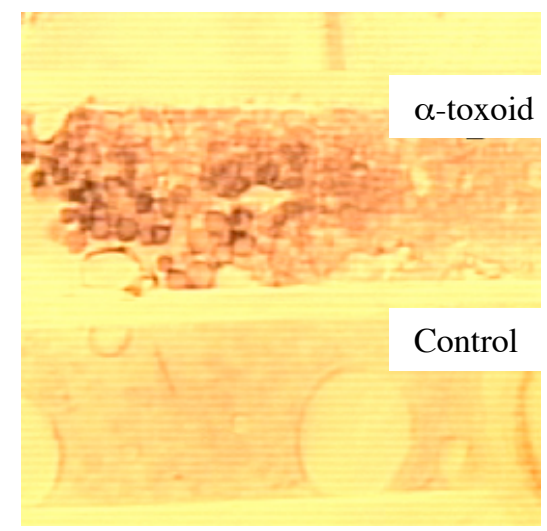

Figure 2.38. Agarose gel as the readout 5 min after introducing the substrate. The channel with is $1000 \mu \mathrm{m}$.

\section{Hydrogel Expansion Sensor}

Another potential readout consists of an expanding hydrogel breaking down a virtual wall. The ability to create a virtual wall within the $\mu$ FT platform is shown in Figure 2.39. The technology is useful for creating chemically separate, but physically close microenvironments. This is typically needed for introduction of reagents into a solution or for combining two carefully prepared microenvironments at a later time (cell microcultures). This is done via the use of a swelling hydrogel. The hydrogel responds to $\mathrm{pH}$, temperature, or just the presence of solution depending on the type of crosslinking. A hydrogel is constructed in one of the microenvironments in close proximity to the virtual wall. Once stimulated, the gel swells reaching over the virtual wall area. The hydrogel moves its surrounding microenvironment with it across the barrier leading to mixing of the two solutions. Figure $2.39 \mathrm{~d}$ illustrates virtual wall 
breakdown. In this case a solution of the $\mathrm{pH}$ indicator phenolphthalein was mixed with a basic solution to cause a purple color upon contact.

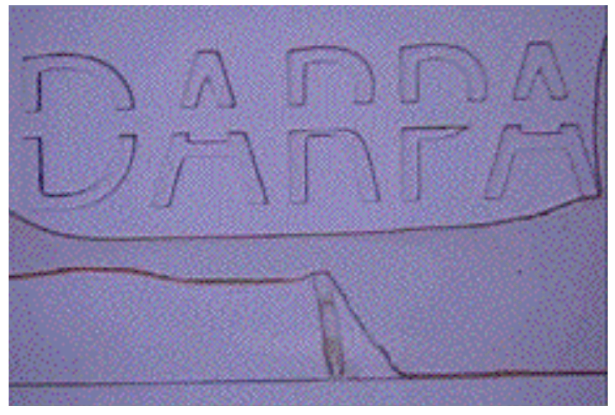

(a)

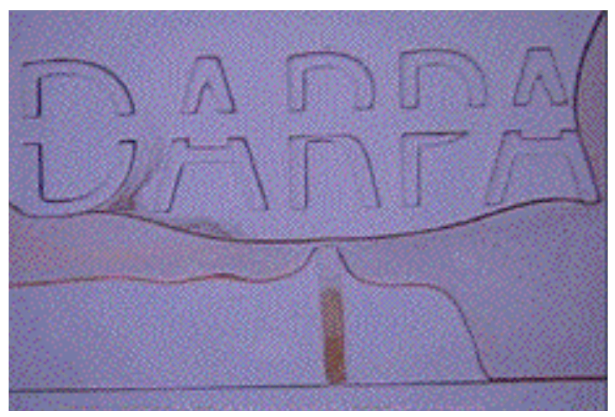

(c)

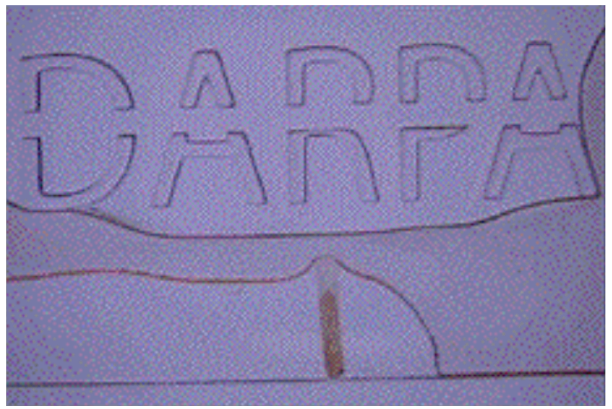

(b)

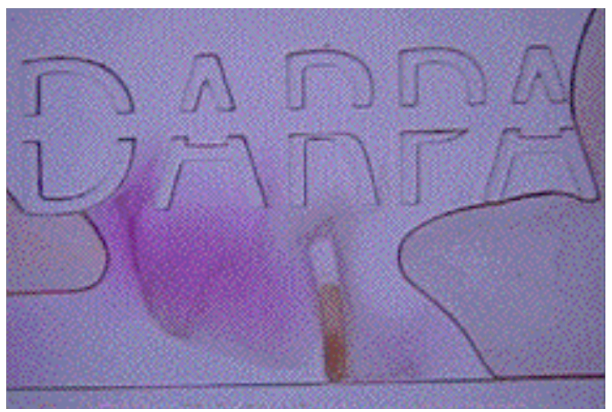

(d)

Figure 2.39. Progression of virtual wall breakdown with a hydrogel using a pH indicator phenolphthalein and a base. (a) Virtual wall separating two fluids, one with a hydrogel arm present. The top fluid is basic, and the bottom fluid has indicator in it. (b) The hydrogel begins to grow towards the other solution across the virtual wall. (c) The hydrogel is shown just before complete virtual wall breakdown. (d) Virtual wall breakdown is shown with pink color from the mixing of $\mathrm{pH}$ indicator and base.

\section{Liposomes}

The layout of a simple glass device used to test the model "signal amplification" from liposomes [7] is shown in Figure 2.40. The device is composed of an emulsion filter and acrylamide posts located downstream, some of which contain dissolvable S-S crosslinkers. Parts of an actual device with uploaded liposomes are shown in Figure 2.41. Liposome complexes are loaded and captured by the emulsion filter. After washing, liposomes are solubilized by addition of detergent (10\% triton), thus unloading the encapsulated reducing agent, TCEP-HCl, downstream. Although incomplete, modest dissolution of S-S crosslinked posts can be seen, some showing tethers (Figure 2.41d and e). 


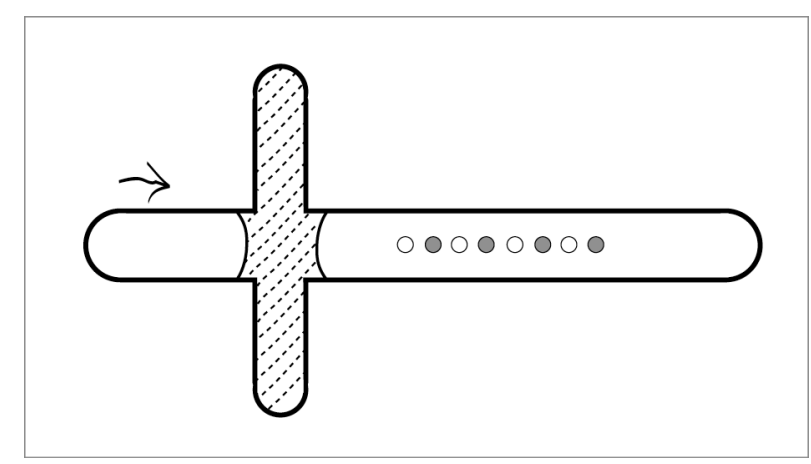

Figure 2.40. Schematic of the microfluidic device. Shaded portion denote the location of the polymerized emulsion filter. Clear circles downstream indicate normal acrylamide gel posts, while gray circles denote disulfide crosslinked posts. Arrow indicates the direction of flow.

Posts are about $500 \mu \mathrm{m}$, and the width of the horizontal channel is $3000 \mu \mathrm{m}$.
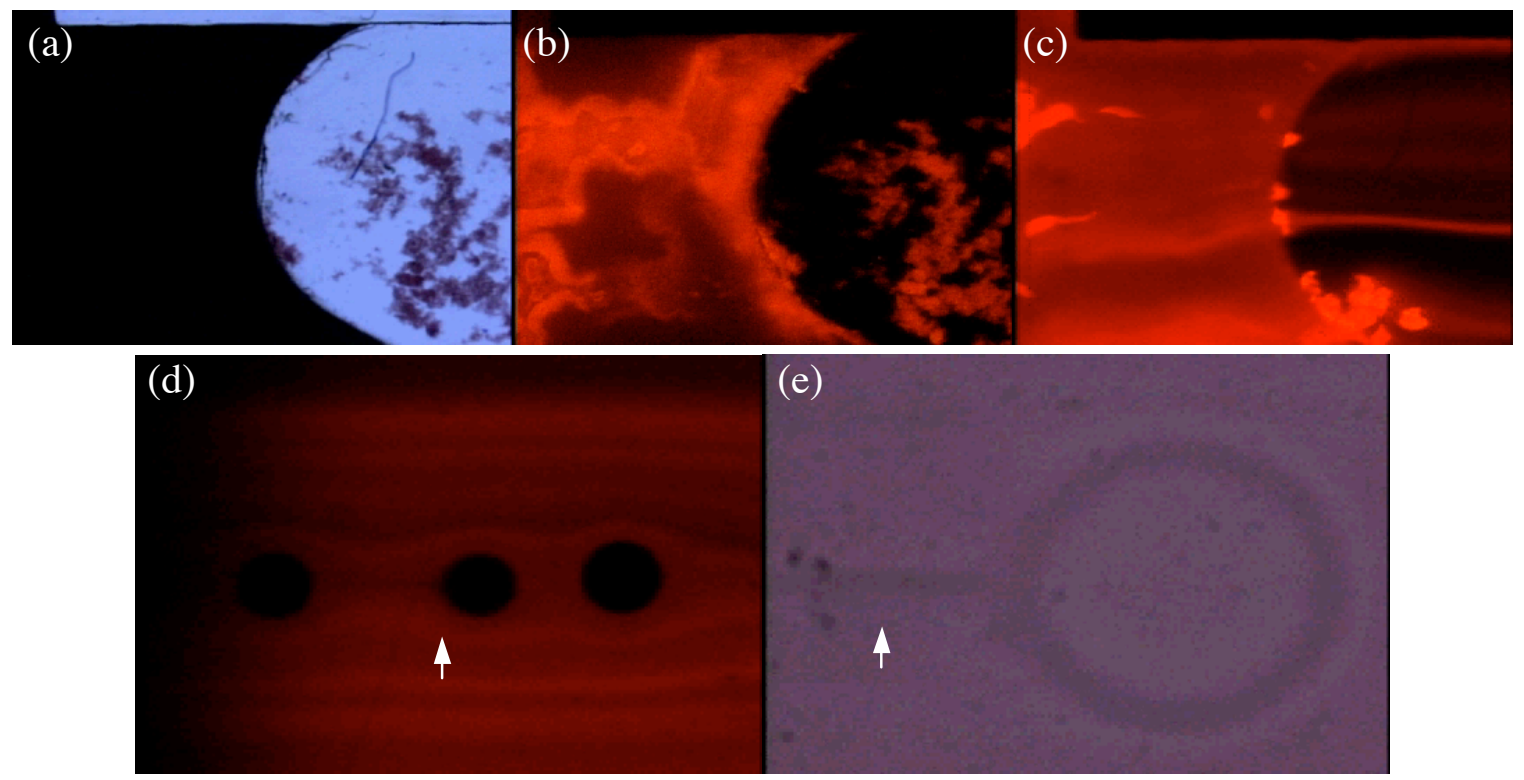

Figure 2.41. Filter with TCEP-HCl encapsulated liposomes and downstream acrylamide gel posts in the channel. Upstream filter seen in white light (a) and in fluorescence $(b)$ where large clumps of vesicles are visible within the filter and on upstream surface. With $10 \%$ triton, vesicles are solubilized (c), unloading encapsulated TCEP-HCl downstream. Downstream, acrylamide gel posts with disulfide crosslinkers slowly dissolve (d, outer two posts are nondissolving, only the center post contains $S$-S crosslinkers) and a tether is formed ( $d$ and $e$, arrows) in direction of the flow (e). Direction of flow is right to left.

The acrylamide gel posts with and without disulfide crosslinkers are examined more closely in a time series (Figure 2.42) following lysis of filter-captured liposome complexes. Normal acrylamide gel posts show no detectable change, while S-S crosslinked posts show increased 
uptake of fluorescent lipid indicating structural disintegration and increase in porosity. The degree of such effect was dependent on the location of the posts in the channel. Upstream S-S crosslinked post shows more significant uptake of fluorescent lipids that correlates to higher uptake of the reducing agent, TCEP-HCl, than a downstream post. This difference may be due to disturbed flow pattern (boundary layers) generated by the upstream posts.

(a)

1

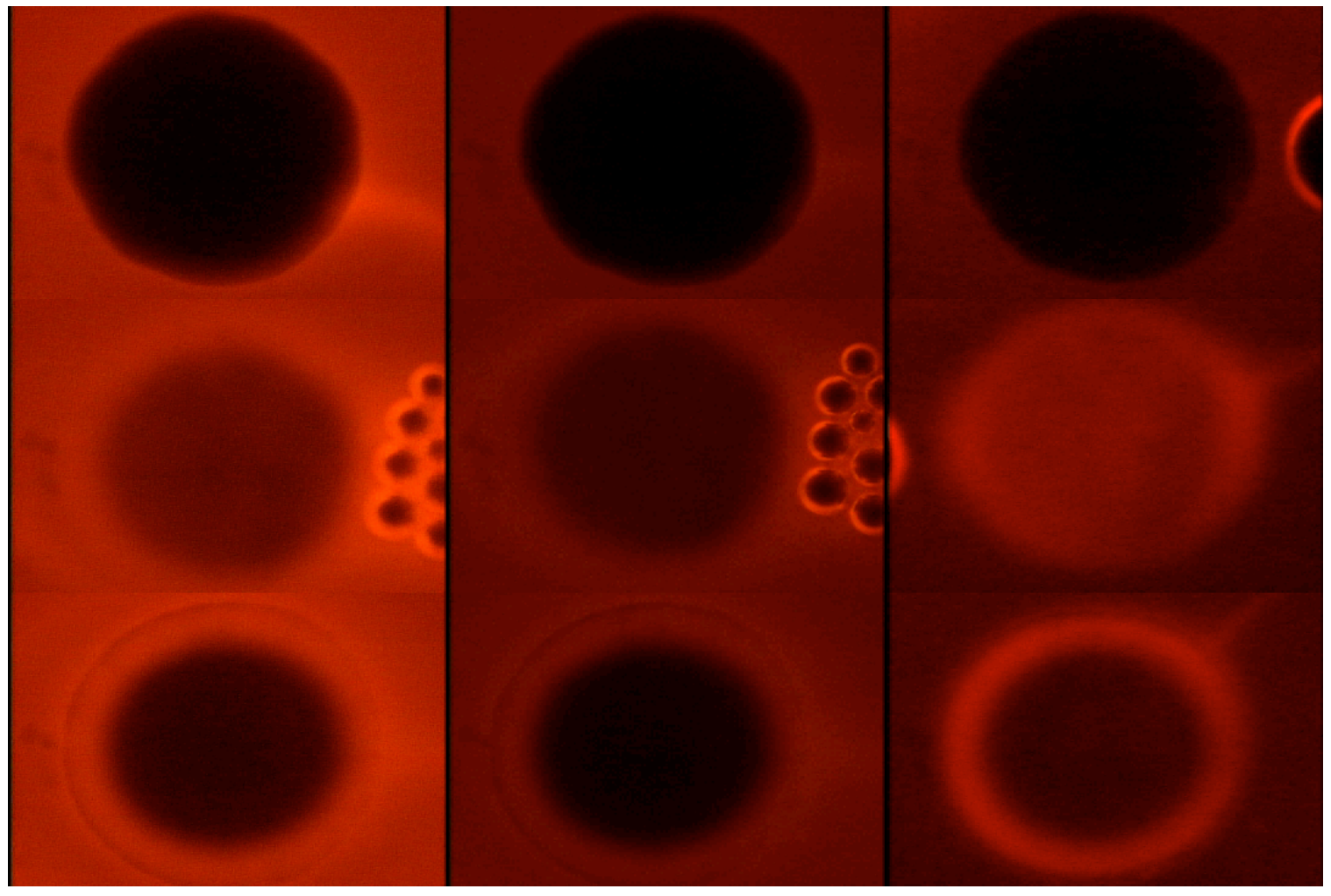

Figure 2.42. Effect of liposome released TCEP-HCl on normal acrylamide post (a) and on disulfide crosslinked posts $(b, c)$ in a microfluidic channel. Posts a and $b$ were next to each other close to the filter, while post $c$ was located downstream. A significant uptake of fluorophorelabeled lipid is seen (b3) which indicate reduction of disulfide crosslinks and dissolution of the gel post. The S-S crosslinked post located further downstream show reduced uptake of fluorescent lipid, which penetrated only the outer edges of the post (c3).

\section{Mixers}

Mixing in microchannels is an important component of biological assays. Both passive and active mixers were fabricated and incorporated into the universal cartridge. 


\section{Passive Mixer}

The passive mixer (Figure 2.43) was fabricated using the layering technique as described previously [13]. This mixer utilized 3D serpentine chaotic mixer geometries previously made with PDMS molding by the Beebe group.

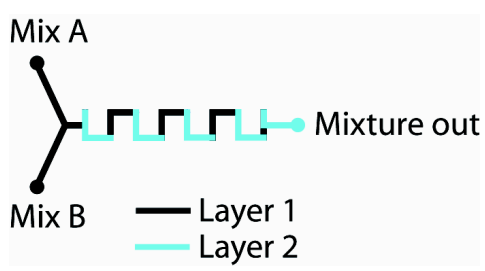

(a)

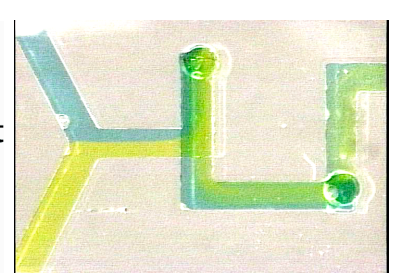

(b)

Figure 2.43. A schematic is shown of a 3D serpentine chaotic mixer (a). Layers 1 and 2 are connected to form a micromixer with blue and yellow dye. The streams start to appear green by the first turn in the channel $(b)$.

\section{Active Mixer}

The active mixer [17]was constructed using a magnetic "stir bar" made from a fabricated metal "blade". The channels were polymerized around the blade forming the mixing chamber (Figure 2.44a). The blade was held into place by forming a post inside the hole of the blade as shown in Figure 2.44b. Placing the device on top of a magnetic stirrer (commonly found in most research labs) activates the mixer. The mixer is shown in the off mode (Figure 2.45a), the on mode (Figure 2.45b) and at such high flow rates that incomplete mixing is occurring (Figure 2.45c)

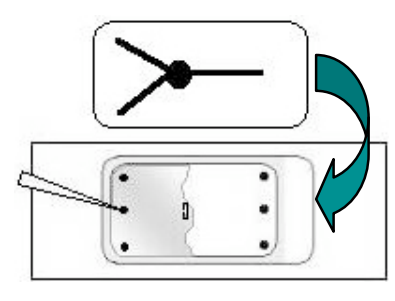

(a)

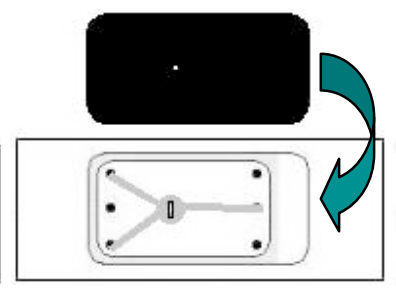

(b)

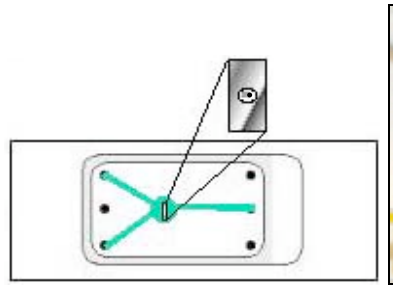

(c)

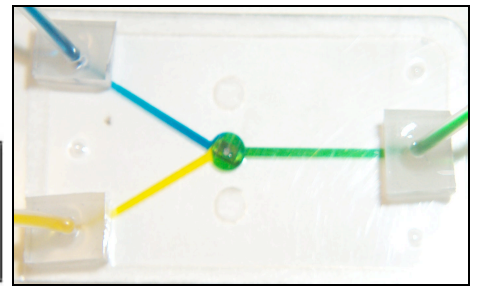

(d)

Figure 2.44. A magnetic mixing device is made by positioning a blade inside a cavity, filling the cavity with prepolymer, and exposing the device to UV to form the channel network (a). A 275 $\mu \mathrm{m}$ mask is placed over the mixing chamber $(b)$, and a post is polymerized inside the hole of the blade (c). A completed device is shown (d). 


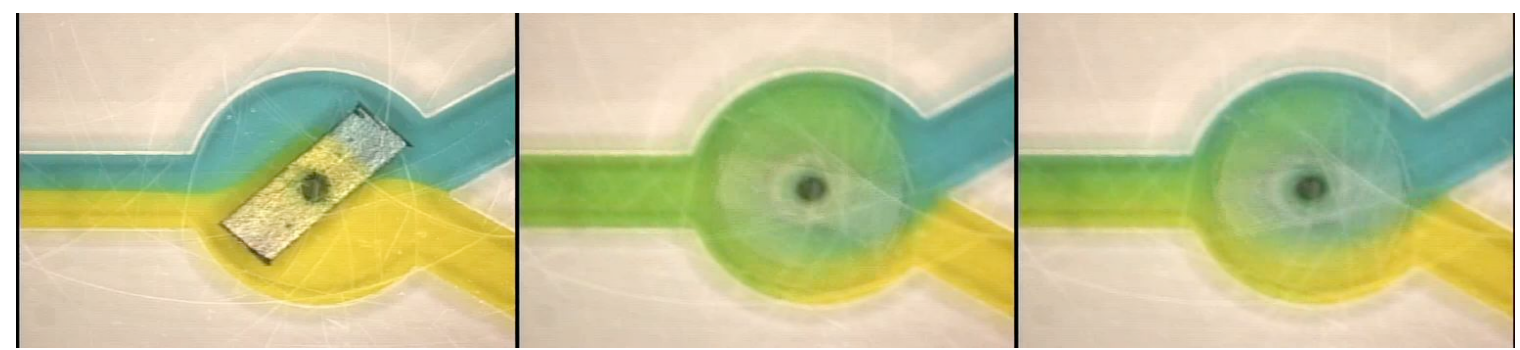

Figure 2.45. The $2.5 \mathrm{~mm}$ device, shown before mixing begins (a), during mixing at a flow rate of $2 \mathrm{~mL} / \mathrm{min}(\mathrm{b})$ and at a flow rate too high $(8 \mathrm{~mL} / \mathrm{min})$ for the mixer to mix efficiently $(\mathrm{c})$. Channel width is approximately $1 \mathrm{~mm}$.

Unlike most active mixers, this device does not require expensive materials or time-consuming cleanroom fabrication techniques. The blade is actuated by a common stir-plate, giving the user a convenient method of controlling the mixing in the device. The ease of fabrication lets the user customize the mixer so that the mixer operates efficiently within the constraints of the channel network. This mixer can be easily adapted to meet the required conditions simply by varying the length of the blade, the speed of the stir plate, or the channel size.

\section{Membranes}

The ability to produce stable liquid-liquid interfaces within microchannels, affords an environment in which high surface-to-volume ratios exists, as well as spatial control of fluid location and flow. This provides chemists the opportunity to conduct chemical reactions involving liquid-liquid interfaces. There are several systems involving liquid-liquid interfaces that have been targeted for transfer into microfluidic systems- interfacial polymerization, phasetransfer reactions, and chemical extraction. Successful implementation may lead to the production of specialized membranes, reactors, and purification systems within the micrometer scale. The ability to form stable liquid-liquid interfaces also provides the unique opportunity to observe the interfacial area under differing fluid flow (con- and counter-current). Focus has been applied to phase-transfer reactions and chemical extraction under con- and counter-current flow.

\section{Biological Membranes}

Interfacial polymerization was used to create permeable/semi-permeable membranes. These membranes acting as biological sensors were fabricated within the microfluidics platform. These membranes are thin, lowering the diffusion path length of the agent to be detected (ie. large enzymes, toxins and proteases). Exposure to a chymotrypsin solution did not lead to a complete dissolution of the membrane, but an increase in porosity was observed. This can be visualized by utilizing a methanol solution containing a dye. The membrane was exposed to a buffered chymotrypsin solution at $37 \mathrm{C}$ for 1 day. The channel was rinsed in methanol and dried. A 
control experiment was also conducted in the same manner, except the membrane was exposed to a buffered solution without chymotrypsin. After rinsing and drying, a methanolic dye solution was added to the hydrophobic side of the channel. Without a membrane, the solution will immediately cross from the hydrophobic side to the hydrophilic side, filling all four sides of the $\mathrm{H}$-channel. Incorporation of the membrane provides a physical barrier, preventing spontaneous crossover to the hydrophilic side, filling only half of the channel. If the porosity of the membrane changes considerably, the methanolic solution should permeate through the membrane and cross over to the hydrophilic side, filling the entire $\mathrm{H}$-channel. This provides a visual detection of exposure to chymotrypsin. It was found that the membrane exposed only to buffer solution did not change, and prevented the permeation of solvent (Figure 2.46). The membrane exposed to chymotrypsin became porous and could not contain the methanol solution. As a result, the methanol solution permeated through the membrane and crossed over to the hydrophilic side, filling all sides of the H-channel (Figure 2.47). It was found that membranes utilizing this peptide sequence did not completely dissolve.

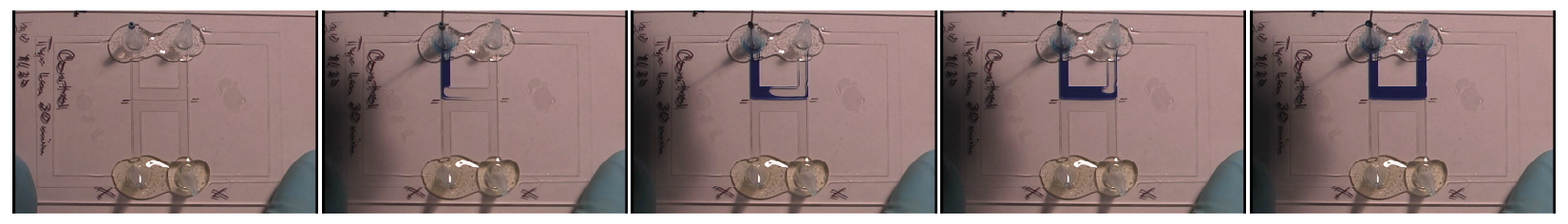

Figure 2.46. Sequential images of dye solution (1\% wt. Brilliant Green in MeOH) filling the Hchannel. This membrane was exposed to a buffer solution not containing chymotrypsin. The channel was initially dry and empty prior to filling. Notice the directed filling by the membrane, indicating no change in the membrane porosity.
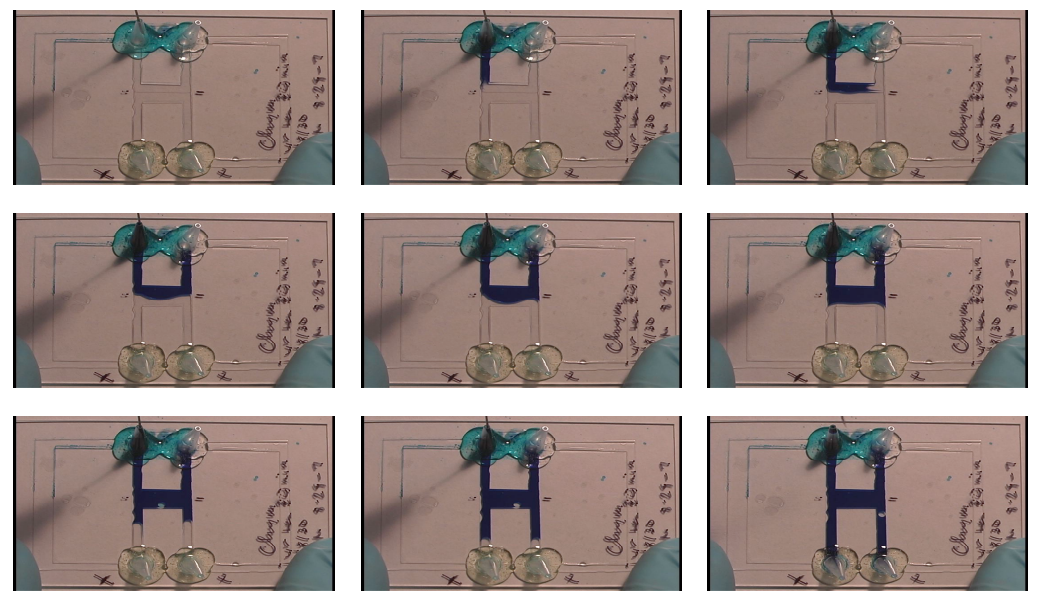

Figure 2.47. Sequential images of dye solution (1\% wt. Brilliant Green in MeOH) filling the Hchannel. This membrane was exposed to a buffer solution containing chymotrypsin. The channel was initially dry and empty prior to filling. Notice the solution permeating though the membrane indicating a change in the membrane porosity. 
Using a shorter peptide sequence (below), exposure to a chymotrypsin solution lead to a complete dissolution of the membrane (Figure 2.48).

\section{Shorter Peptide Sequence:}
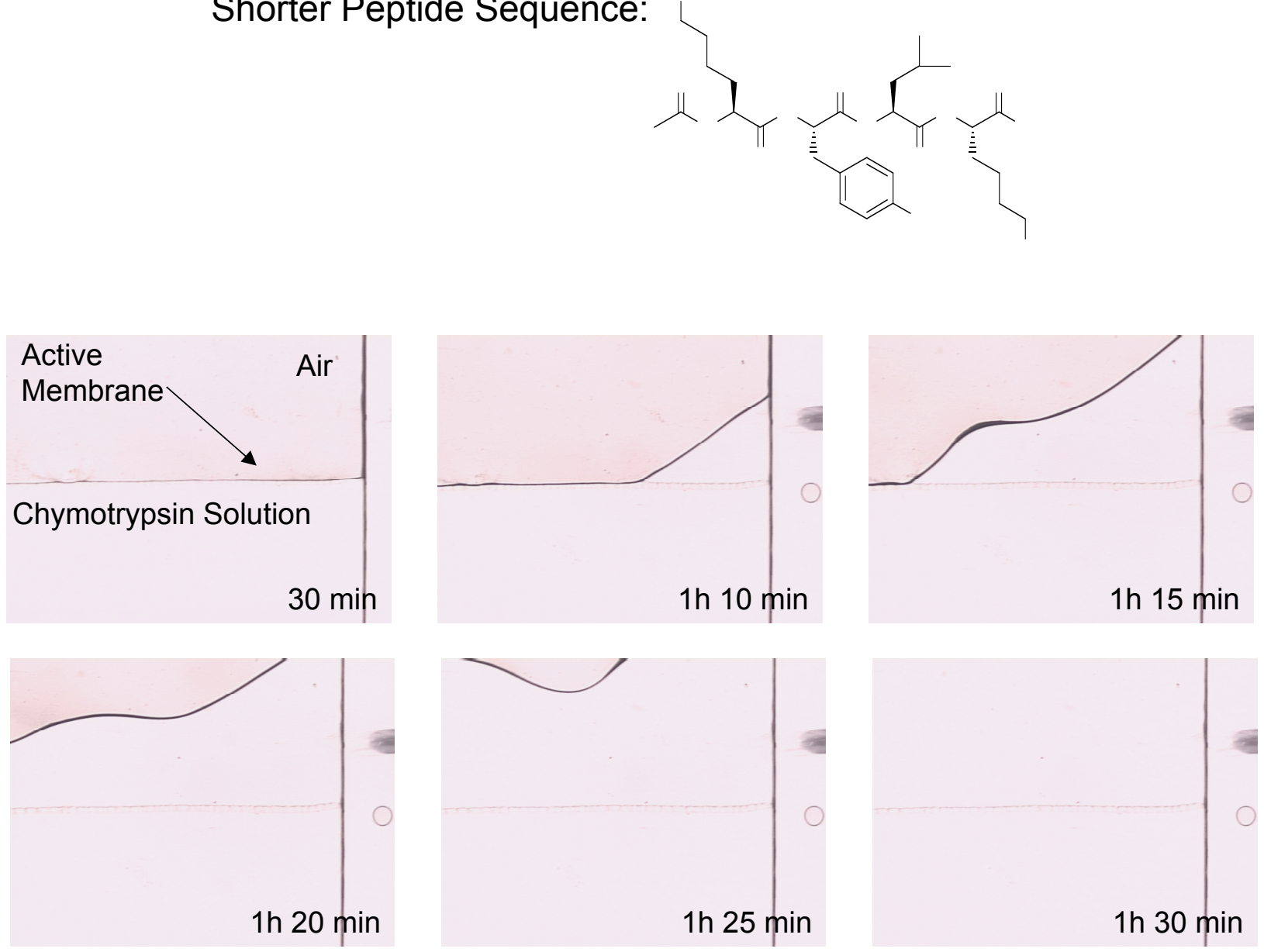

Figure 2.48. Sequential images of a membrane completely dissolving at room temperature.

\section{Pumps}

There are several kinds of pumps which are used to displace fluid. These pumps are both passive and active. A brief description of these types of pumps follows.

\section{Fluid Dispenser}

The ability to manipulate fluids in microchannels is very important for any assay development. The concept for the design of the fluid pusher [18] is shown in Figure 2.49. PDMS will be used to create the micromolded pumping and actuation chambers. The two layers will be identical so 
they line up and do not collapse. The support structures have different functions in the two layers. Support structures in the bottom layer will prevent the PDMS membrane from prematurely collapsing. These same supports in the top layer guide the prepolymer solution as it fills the chamber and contain the hydrogels to prevent them from moving. The support structures also facilitate correct alignment of the two layers.

The method of fabricating the fluid pushers is shown in Figure 2.49a. Hydrogels are polymerized in the top actuation chamber. The fluid to be pushed will be in the fluid reservoir. When the hydrogels are exposed to basic solutions, they will expand. This will deform the PDMS membrane and reduce the volume of the pumping chamber. The result will be the fluid pushed out of the bottom pumping chamber. The data on pump efficiency, back pressure, and pump volume will all be characterized. A fluid pushing device is shown in Figure 2.49b.

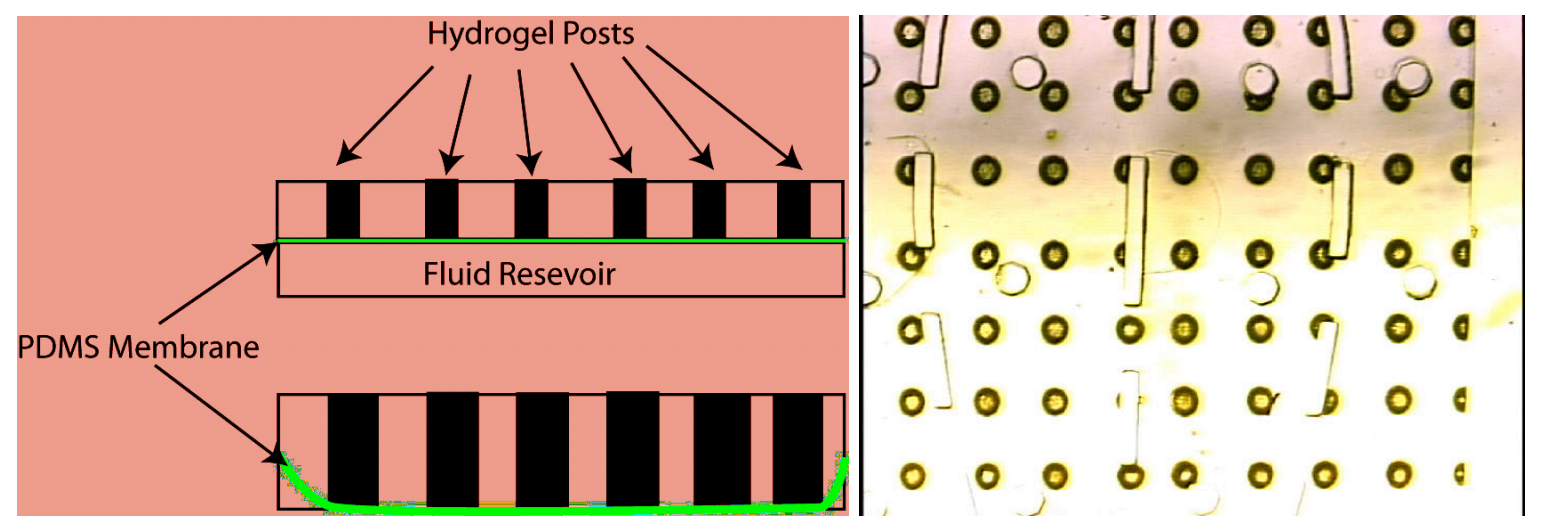

Figure 2.49. a) A schematic (side view) of a fluid pushing design and b) a top view of $300 \mu \mathrm{m}$ hydrogel posts polymerized in the actuation chamber of the device.

Ultimately, this type of pump could be combined with organic sensing methods to create closed loop diagnostic/therapeutic devices. The open-loop non-electronic subcutaneous delivery button will be designed to deliver a compound at a constant rate for eight hours addressing an immediate clinical need (e.g. insulin for a diabetic).

\section{Thumb Actuated Pump (TAP)}

The Thumb Actuated Pump (TAP) is a pump that is actuated by a thumb. The overall schematic of the design is shown in Figure 2.50. Because the force generated by the thumb is large (macro), the device needs to dampen this or have an overpressure vent. The dampening will be accomplished by making the reservoir smaller than the thumb, so when the thumb pushes down, most of the force will be absorbed by the bulk of the PDMS and not the membrane covering the reservoir. However, there will still be enough force to completely deform the membrane and empty the reservoir. 

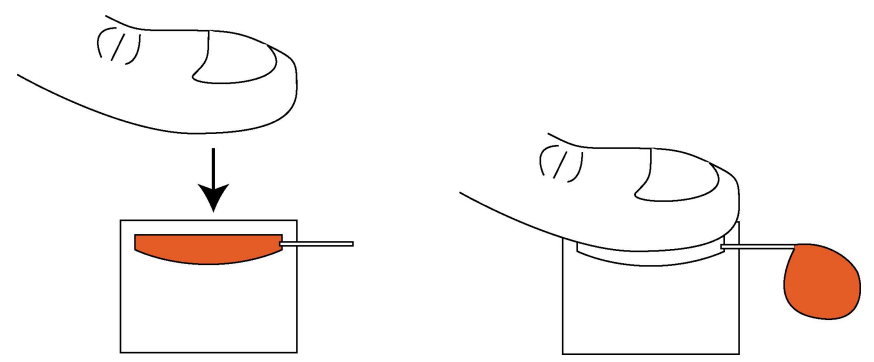

Figure 2.50. Schematic representation of the thumb actuated pump (TAP).

The TAP will be operated with a deformation of the membrane followed by the thumb sweeping along the reservoir. The fluid in the reservoir will need to be pumped through several system components such as a mixer, filter, and channel network. The filter element cannot withstand pressures greater than 15 psi. If the pressure exceeds this value, the filters will delaminate. Pressure vents will be integrated by not pushing the TAP down completely. There will be deliberate regions along the reservoir that will not be taped down. The seal will therefore be the PDMS on the cartridge surface, which will be able to be broken if a critical pressure is reached. The advantage of the TAP is its simplicity, and the TAP is an effective first step in an autonomous pumping device.

\section{Passive Pumping}

We have found that fluid motion in a microchannel results from the surface energy of the liquid in a microfluidic device. Therefore, no external pumps, tubing, connectors, or energy are required to perform the pumping. This eliminates tremendous costs. No reliable, cost effective micro pumps have been developed to date (i.e., none are commercially available). Those that are being developed (i.e., evaporative pumps) are still complex when compared to the pumping concepts described here.

Currently, high precision pumps that can achieve very low flow rates are required to make any microfluidic device functional. Even very expensive pumps (e.g., \$4000) do not provide a constant flow rate (i.e., the flow is never steady). This pumping scheme provides a flow rate smoother than anything currently achievable and provides another feature not currently available - smoothly changing flow. In addition to smooth flow, passive pumping allows highly accurate quantities of fluid to be pumped smoothly through a microchannel in a highly noisy environment (i.e., motion, non-level surfaces).

Current commercial microfluidic devices rely on electrokinetic flow as the pumping mechanism because it is easy to control and incorporate into microfluidic designs. The main drawbacks are that electrokinetic flow denatures biological samples, can adversely affect chemical samples, and requires high voltages. Passive pumping does not rely on electrodes to produce flow, thus eliminating these detrimental effects while at the same time providing an equal, or greater, degree of precision in the control of fluid flow. 
Figure 2.51, illustrates the operation of a passive pump. The Young-LaPlace equation allows one to calculate the pressure inside a drop of liquid (all drops have an internal pressure - that's why they're normally curved on the surface). One consequence of this equation is that a small drop of liquid has a larger internal pressure than a large drop. In Figure 2.51, the small drop has a larger internal pressure than the large drop, and because these two drops are connected via a fluidic channel, the pressure difference causes a fluid flow.

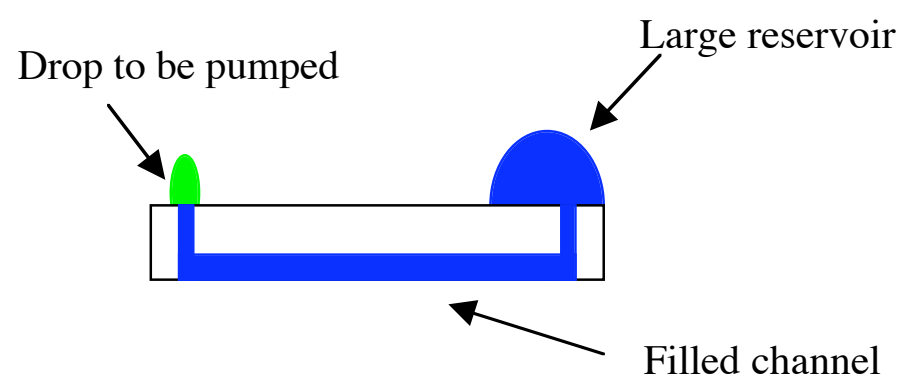

(a)

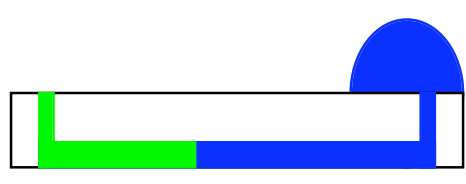

(b)

Figure 2.51. Passive pumping. The surface tension of the green drop in (a) provides enough force to cause the drop to move into the microchannel $(b)$ without any external effort. As long as there is a larger "reservoir" drop somewhere in the microfluidic system, fluid can be pumped from any number of ports to the reservoir drop.

\section{Micropumps}

Microculture systems can provide new insights on cell and cell microenvironment interaction, hence providing a new tool for understanding basic cell biology [19]. An effective culture system should provide nutrients/factors the cells need, and remove wastes while exerting minimum damage to the cells. Traditional spinner/shaker cultures accomplish nutrient delivery to the cell suspension at the expense of generating shear stress which can reduce cell viability. A peristaltic pumping microdevice built in PDMS has been tried on E. coli with a survival rate 94\%. Animal cells are more sensitive to shear stress than bacteria and may require more gentle pumping methods. Development of a micropump to provide recirculating flow for the culture of Fall armyworm ovarian cells (Sf9) (shear stress sensitive) in microchannels was demonstrated The device and experimental set up are shown in Figures $2.52 \& 2.53$. 


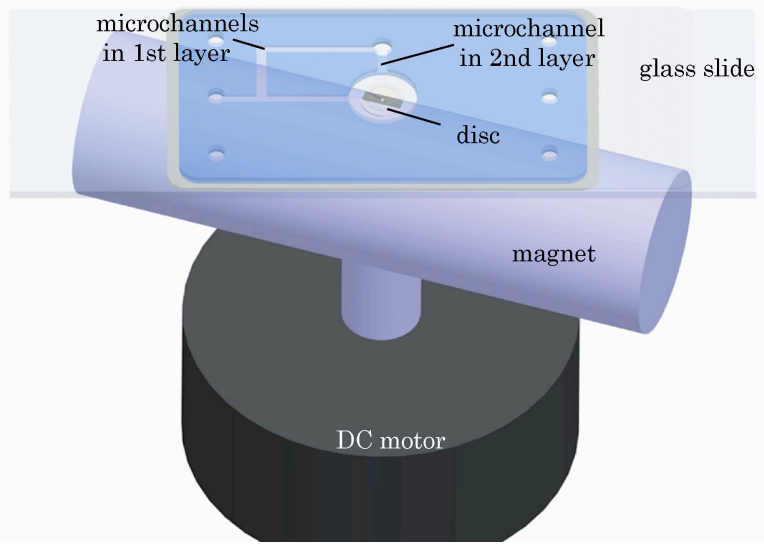

Figure 2.52. Mechanical model of the micro pump. The disk actuator is driven by an external DC motor through magnetic coupling.

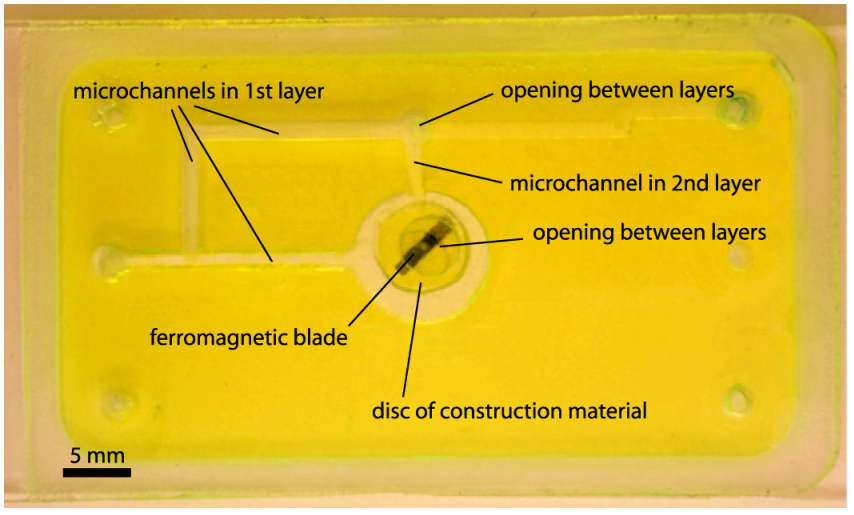

Figure 2.53. Rotary pump built in a two-layer microtectonics platform. The actuator consists of a ferromagnetic bar embedded in a polymer disc, placed in the first layer.

The pump is a variation on a traditional impeller pump where a disk is used in place of the impeller (a traditional impeller design was used for comparison). When the disk rotates, it transfers momentum to the layer of liquid in contact with it. This layer tends to rotate with the disc due to adhesion, and at the same time, tends to move away from the axis of rotation due to centrifugal force. The momentum is then transferred to the different layers of the liquid due to viscous drag, and produces a local non-pulsating flow. Figure 2.54 (a) shows the axial component of the flow patterns in a cylinder with a rotating disk inside. 

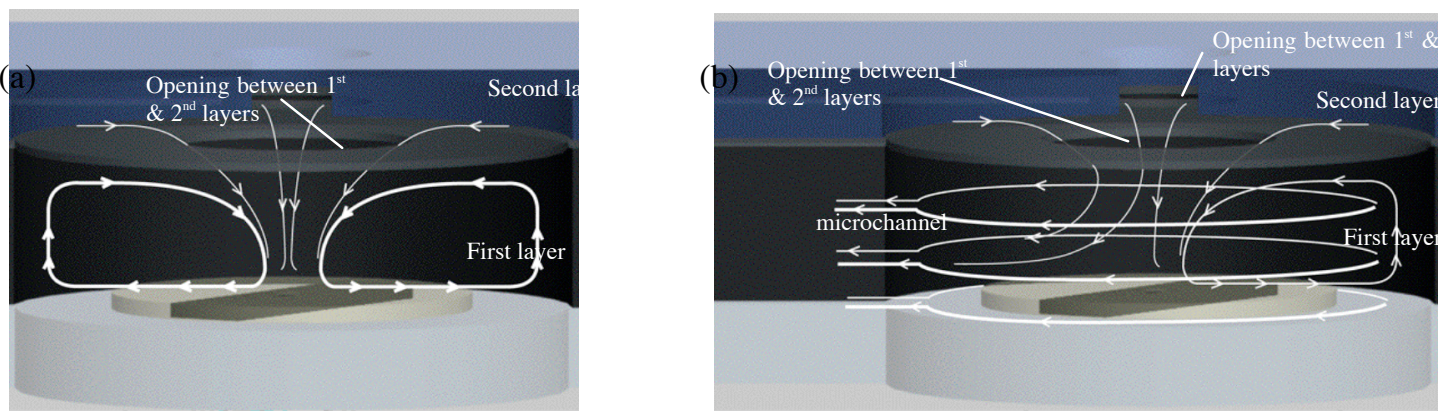

Figure 2.54. Diagram showing the path flows in (a) closed cylinder with only an opening at the top (b) cylinder with an opening at the top and another one in the left part (micropump). A nonpulsating net flow is created due to viscous drag, between layers of the fluid and adhesion to the surface of the rotating disk.

The flow rate vs. rotational frequency for both pump designs (disk, impeller) was measured via visualization of a dye moving through the channel (Figure 2.55). A cell recirculation experiment was conducted in order to visualize the effects of the shear stress of the pump rotating at $10 \mathrm{~Hz}$. After 23 minutes of recirculation, no lysed cells were found by Typan Blue staining. At $50 \mathrm{~Hz}$, pieces of some cell lysis were observed. Cell lysis occurred at all speeds in the impeller pump design.

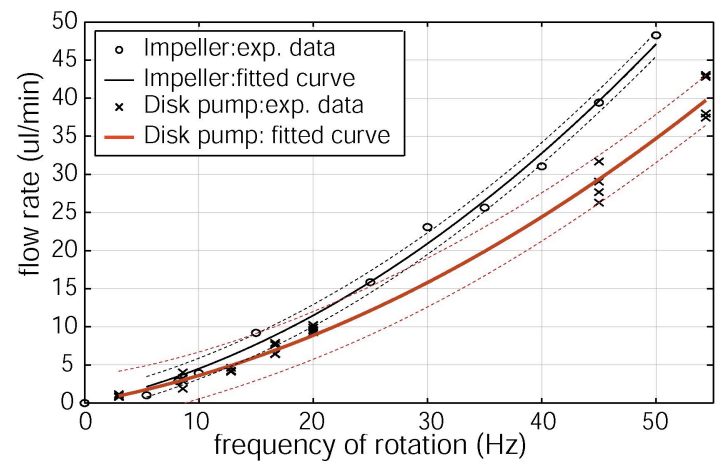

Figure 2.55. Flow rate versus frequency of rotation of the disc pump and of an impeller pump.

Figure 2.56 shows two models for calculating the maximum shear stress produced (a) in the disk type micropump, and (b) in an agitated vessel with an impeller used in macro scale culture systems. According to the model in Figure 2.56 (b), the maximum shear stress is $6 \mathrm{dynes} / \mathrm{cm}^{2}$, which is very similar to the one calculated for the disk micropump at $10 \mathrm{~Hz}\left(5.6 \mathrm{dynes} / \mathrm{cm}^{2}\right)$, in Figure 2.56 (a). The cells are only exposed to the rotating disk for a small percentage of each cycle through the system. The shear stress can be reduced further by decreasing the frequency of rotation of the pump. Table 2.5 summarizes the results. 
(a)

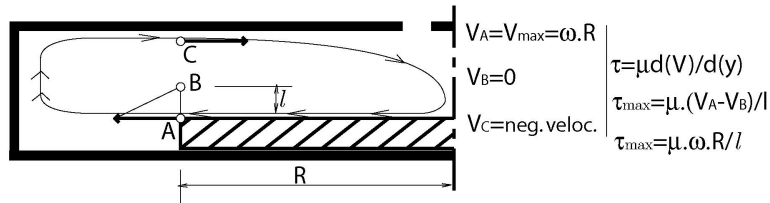

(b)

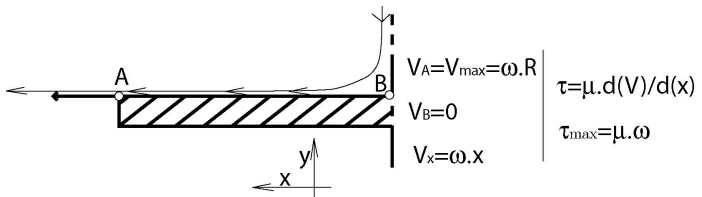

Figure 2.56. Velocity 'V'and max. shear stress '_max' in (a) upump (b) impeller in a big flask. In case $(a)$ assuming that $\mathrm{l}=\mathrm{d} / 2=0.275 \mathrm{~mm} / 2$, with a frequency of rotation $\mathrm{f}=10 \mathrm{~Hz}$, then ${ }_{-\max }=5.6$ dynes $/ \mathrm{cm}^{2}$. In case $(b)$ the maximum gradient of $\mathrm{V}$ is in the $x$ direction, which means that ${ }_{-\max }$ is only function of the frequency of rotation.

\begin{tabular}{|l|c|c|c|c|}
\hline & Type of flow & $\begin{array}{c}\text { Calc. Shear Stress } \\
\text { at } 10 \mathrm{~Hz}\end{array}$ & Lysed cells at $10 \mathrm{hz}$ & Lysed cells at 50 hz \\
\hline Disk & Non-pulsating & 5.6 dynes $/ \mathrm{cm}^{2}$ & None observed & Medium \\
\hline Impeller & pulsating & NA & High & High \\
\hline $\begin{array}{l}\text { Closed } \\
\text { flask } \\
\text { w/agitation }\end{array}$ & pulsating & 6 dynes $/ \mathrm{cm}^{2}$ & Minimal & NA \\
\hline
\end{tabular}

Table2.5. Comparison between pumping methods for insect cells culture.

\section{Hydrogel Microstructures}

While exploring the many advantages of the techtonics platform, a method of fabricating micron sized structures outside the microchannel environment was discovered. Ethyl vinyl acetate (EVA) tubing is a commercially available tubing that can be stretched and heat cured back to its original state. The tubing was stretched (inner diameter approximately 150-200 microns) and filled with construction material. The tubing was then exposed to UV light for 30 seconds. After exposure, the tubing was heated and allowed to shrink back to the original shape (inner diameter approximately 600 microns). Instead of producing a thin thread, the construction material actually shrank inside the tubing (Figure 2.57a) forming a coiled structure (Figure 2.57b). A SEM of the material formed inside the tube revealed a sinusoidal structure (Figure $2.57 \mathrm{c})$. 


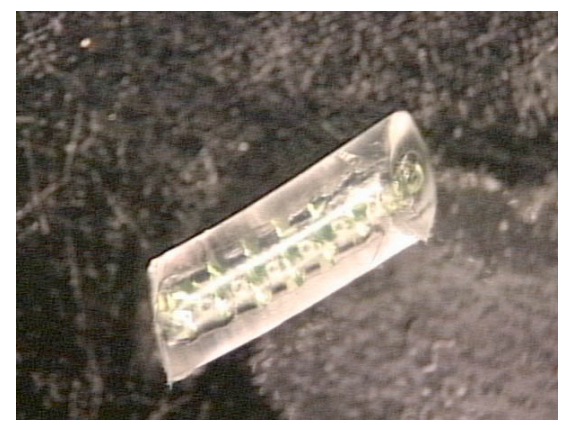

(a)

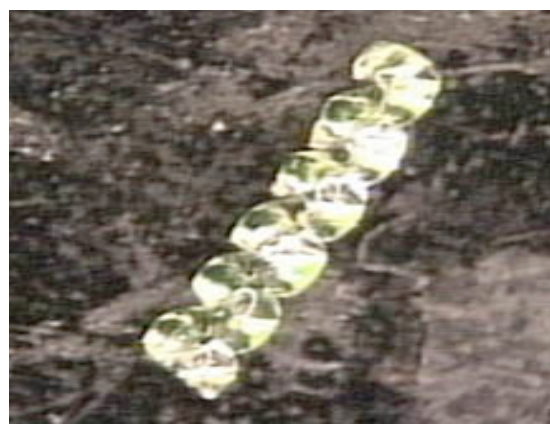

(b)

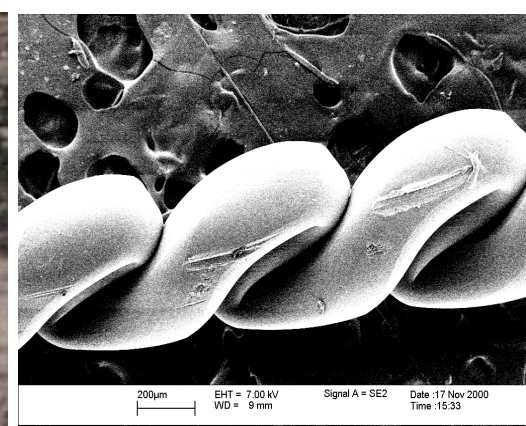

(c)

Figure 2.57. EVA tubing with construction material coiled inside (a) and after being removed from the tubing (b). An SEM image of the coil (c) reveals a sinusoidal shaped structure.

The same technique was also used on the responsive hydrogel material. That material also formed structures when exposed to heat, but the coils were much looser in nature, and the EVA tubing actually shrank back around the hydrogel material (Figure 2.58a) creating a deformed EVA tubing in the shape of the hydrogel structure. This type of coil is in contrast to the construction material which formed inside the cylindrical tubing (as shown in Figure 2.57a). Since the material responds by swelling to an increase in $\mathrm{pH}$, the structures were exposed to acids and bases to see how they would react to the changes in $\mathrm{pH}$. When these structures are exposed to acidic solutions (in this case $1 \mathrm{M} \mathrm{HCl}$ ) and also to DI water, the structures expand slightly due to the uptake of water (Figure 2.58b). The responsive material was then exposed to a basic solution, and the material swelled dramatically as shown in Figure 2.58c (a similar tail was on the right side of the structure but broke off when extracted from the vial).

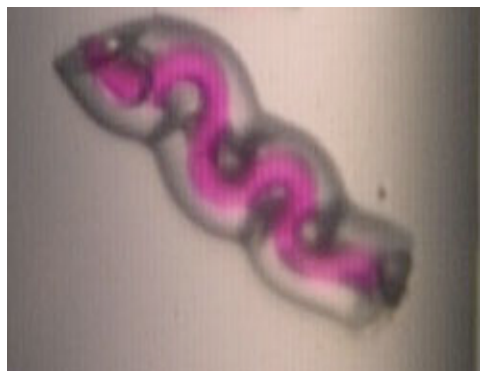

(a)

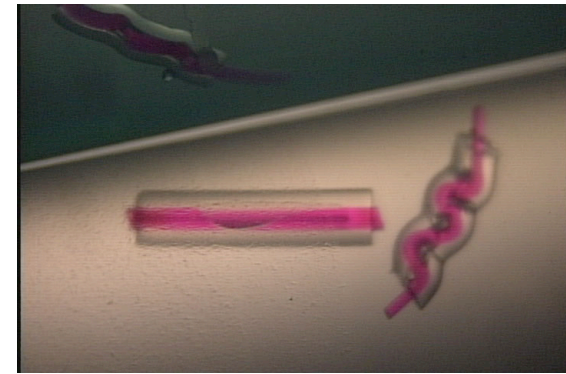

(b)

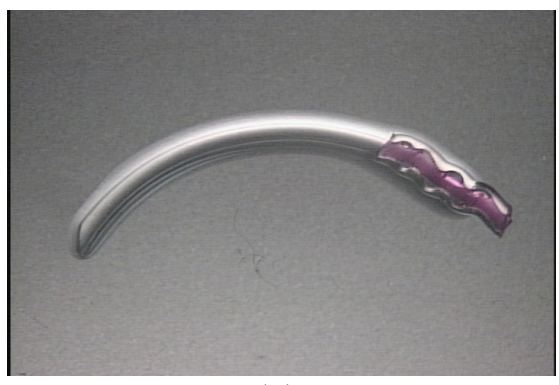

(c)

Figure 2.58. EVA tubing formed around the responsive hydrogel material inside (a). The responsive hydrogel in basic solution initially $(b)$ and after 30 minutes $(c)$.

To make threads, EVA tubing is stretched, filled with responsive material, exposed, and cut into 1 to $2 \mathrm{~cm}$ pieces (Figure 2.59a). The pieces of tubing are placed on a glass slide and cured on a hotplate (Figure 2.59b), and the resulting pieces of hydrogel material are then pulled from the expanded EVA tubing (Figure 2.59c). This technique produces roughly cylindrical threads of 
responsive hydrogel material that is approximately 150 to 200 microns in diameter and free from the EVA tubing.

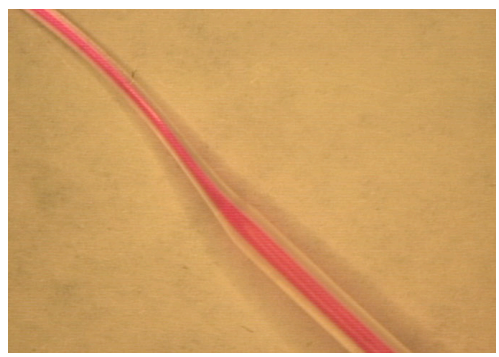

(a)

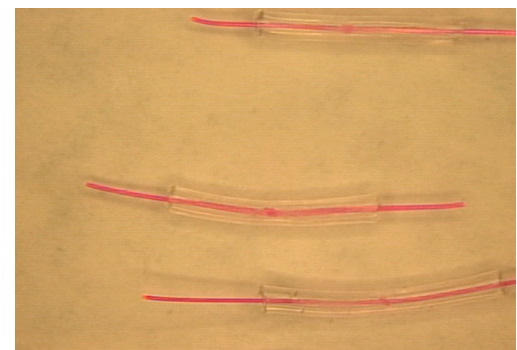

(b)

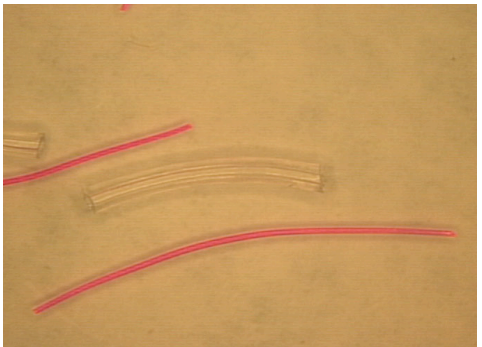

(c)

Figure 2.59. The EVA tubing is cut into pieces (a) and heated on top of a piece of glass (b), to form relatively straight pieces of responsive hydrogel material (c).

Once an easy method of producing threads was found, a number of them were produced. Since the threads were initially brittle, they were soaked in an acidic solution to allow them to rehydrate (water could also have been used). The threads were then carefully woven together using tweezers to manipulate the threads and a magnifying glass to generate an under/over weave (Figure 2.60a). The weave was then allowed to dry out (Figure 2.60b) and then re-hydrated with solution (see Figure 2.60c). After exposure to the basic solution, the threads began to swell even though the weave itself was unconstrained (Figure 2.60d). From the response of the hydrogel material, the fabric began to close up its weave. Responsive hydrogel weaves may have many applications including wound healing and responsive clothing [20].

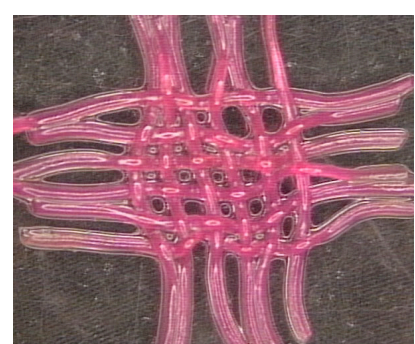

(a)

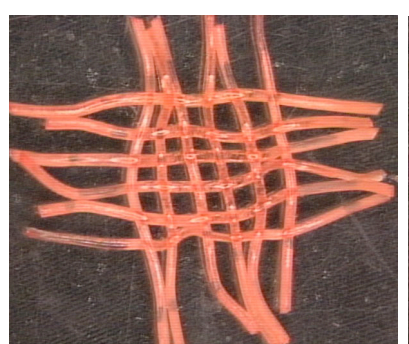

(b)

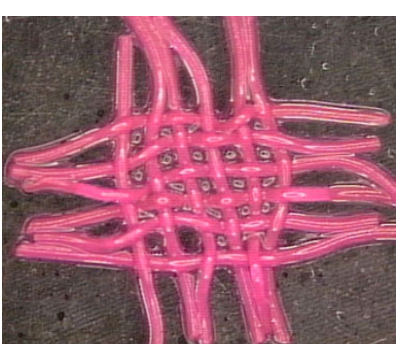

(c)

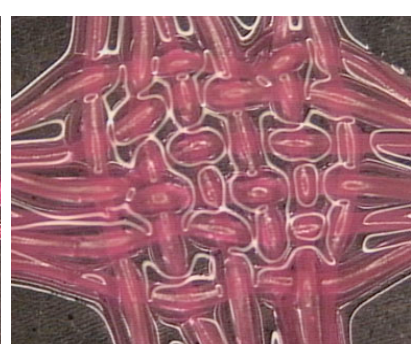

(d)

Figure 2.60. The threads were soaked in an acidic solution and woven into a 6 by 6 grid (a), allowed to dry out (b), re-hydrated with solution (c), and then exposed to base and allowed to swell for 30 minutes $(d)$. 


\section{Chapter 3 Materials Modeling}

\section{1-D Model}

A chemical and mechanical model to predict the swelling kinetics of hydrogels was developed [21]. This model was validated against experimental data that was published in the literature. When the model was compared to experimental data of the hydrogels used for this project, it didn't accurately fit the data. The model was adjusted to include the transport of buffer ions in the chemical diffusion equation. A new model was developed and implemented that investigated the swelling kinetics of hydrogels in buffered solutions. The inclusion of buffer terms was critical to accurately predict the swelling characteristics. A one-dimensional model was developed to investigate equilibrium swelling and deswelling of the hydrogels (Figure 3.1). This model matches the experimental results of the $300 \mu \mathrm{m}$ gels very well but doesn't accurately model gels of smaller diameters. It is thought that as the dimensions decrease, the mechanical response becomes critical and must be included in the model.
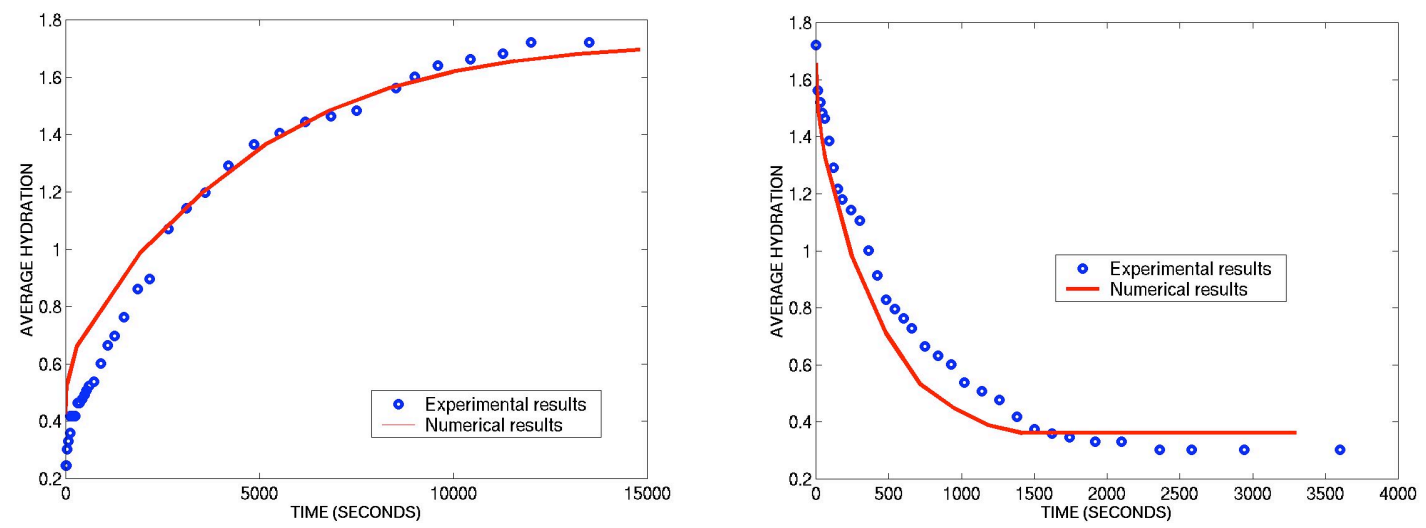

Figure 3.1 . A comparison (a) is shown of experimental and numerical swelling of a $300 \mu \mathrm{m}$ hydrogel. A similar comparison (b) is shown of experimental and numerical deswelling of 300 um hydrogel.

The chemical diffusion equation and mechanical equation were then coupled to model the swelling and deswelling kinetics of $\mathrm{pH}$ sensitive hydrogels in buffered solutions. Experiments were performed on gels of diameters: 300,200 and $150 \mu \mathrm{m}$ to validate the numerical model. The gels were equilibrated in buffered solutions of $\mathrm{pH} 3$ and then put in a bath of $\mathrm{pH} 6$ to study the swelling kinetics. The reverse was done for studying deswelling kinetics. In Figure 3.2, which shows the comparison for the $150 \mu \mathrm{m}$ gels, there was good agreement between the numerical values and experimental results. The chemical diffusion equation gives the rate of the solvent into or out of the gel based on concentration difference, whereas the mechanical equation takes in to consideration the stresses generated inside the gel due to solvent flux. The addition of the 
mechanical contribution helps to predict the swelling and deswelling behavior of the smaller diameter gels where mechanical response becomes critical. Figure 3.2 also shows the Hydration (Ratio of volume of fluid to total volume of gel) predicted by the buffered coupled equation changes slower (although slightly) with the added mechanical component when compared to that predicted by the chemical diffusion equation.

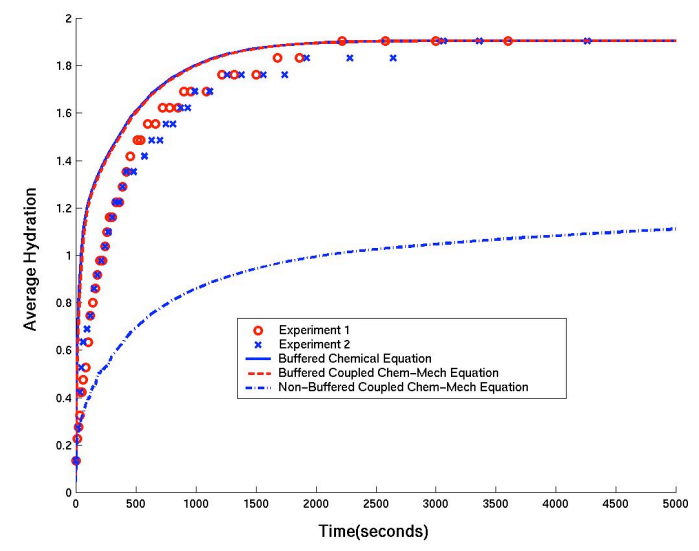

(a)

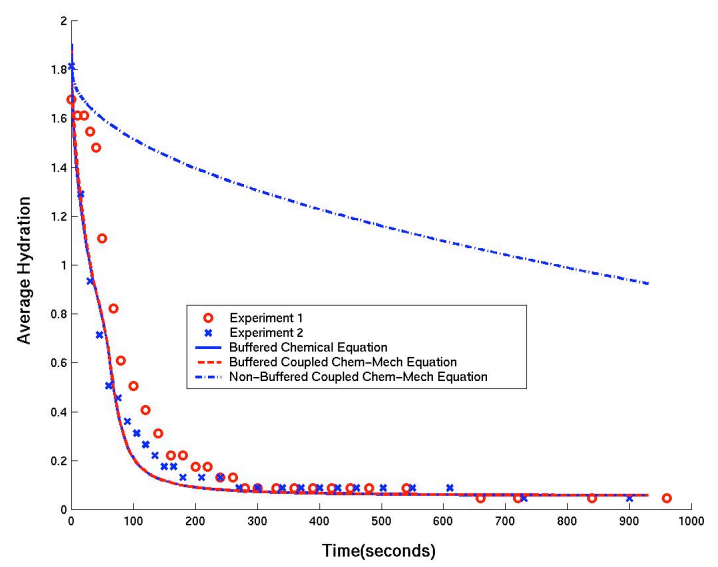

(b)

Figure 3.2. Swelling (a) and deswelling (b) of $150 \mu \mathrm{m}$ gel shows good agreement between the numerical and experimental data.

\section{Modified 1-D Model}

Previously, a one dimensional mechanical equilibrium equation was solved to get the deformation of the gel, however this is an approximation. Therefore, the equilibrium model for $\mathrm{pH}$-sensitive hydrogels was modified. The Nernst-Planck equation was coupled with the Poisson equation to solve the steady state concentrations of the different ions inside the gel. The new model solves a modified mechanical equilibrium equation, which is an exact representation of the hydrogel. The gels, which are cylindrical in shape, are restricted to deform along the height. Hence, the deformation of the hydrogels can be modeled by solving a plane strain problem, as shown in Figure 3.3. The strain along the vertical direction is zero, hence, all cross-sections of the cylinder are identical. Therefore, the final deformation equation is that of a circular section subjected to outward radial pressure, which has a simple analytical solution. The new model was applied and compared with experimental data with good agreement.

The expression for Hydration (which is defined as the ratio of the volume of fluid to solid in the gel) has been expressed in terms of the strain at a point. This is an important development, since, now for any geometry, the Hydration at a point can be specified, and a proper mechanical deformation equation can be obtained for that shape. It is also used in the kinetic model for hydrogel swelling, hence, one can continue to use that model by using Hydration values calculated by the equilibrium model. 


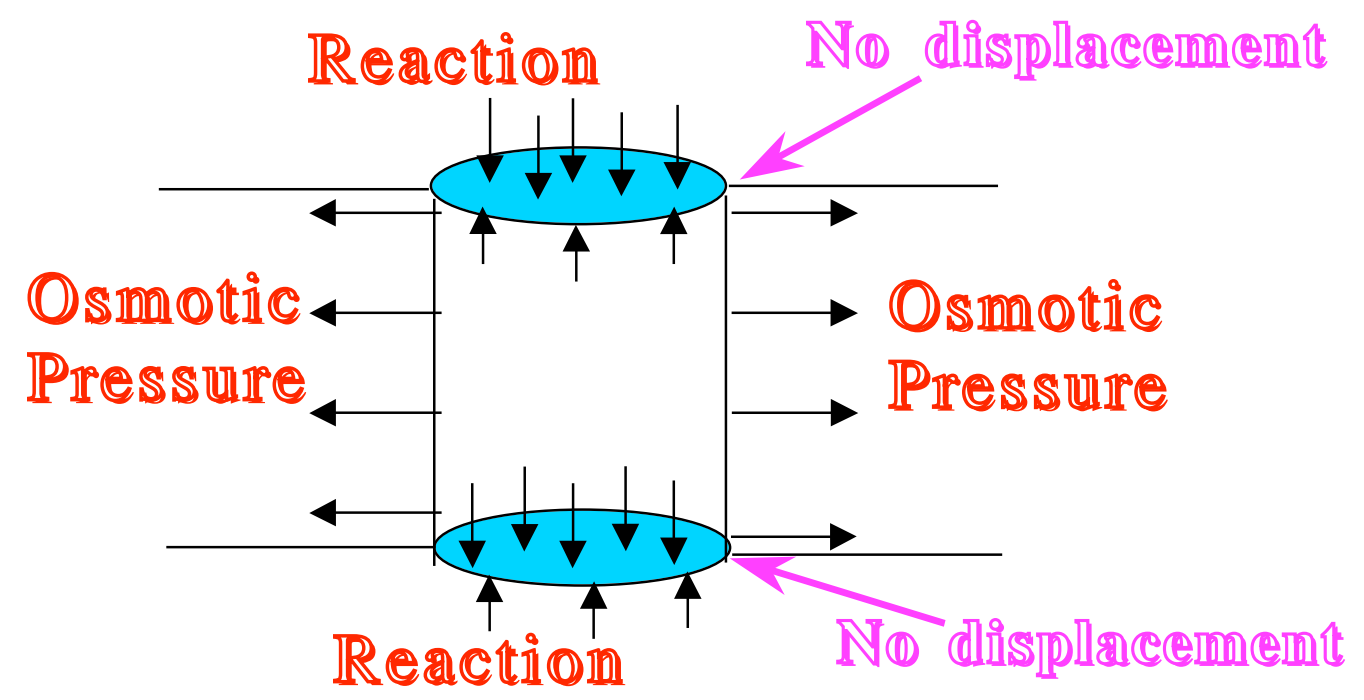

Figure 3.3 Hydrogel deformation model.

Figure 3.4a shows the comparison between the experimental data and the numerical simulations for two gels of different dimensions. The final diameter of the gel is calculated. The model allows us to calculate the forces exerted by the gels at the top and bottom surfaces during the swelling process. The variation of these forces with the solution $\mathrm{pH}$ is shown in Figure 3.4b.

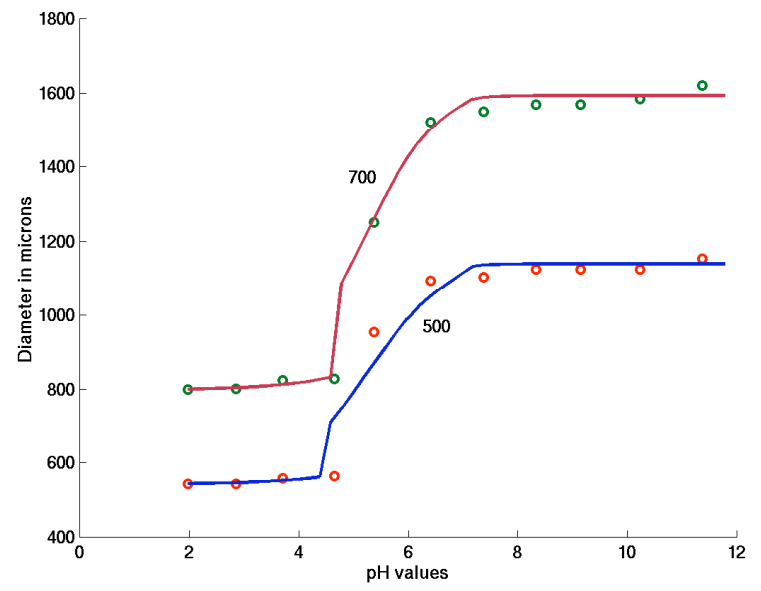

(a)

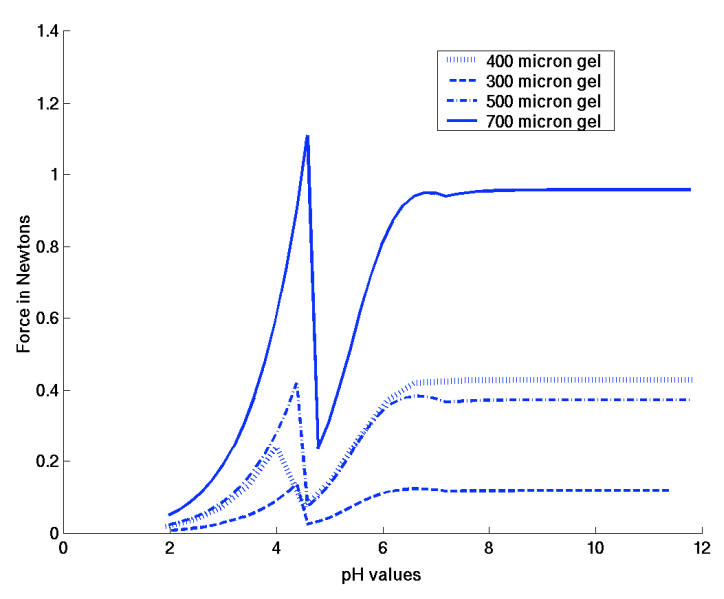

(b)

Figure 3.4. a) Swelling of 500 and 700 micron gels from $\mathrm{pH}=2$ to 12 , and b) forces exerted by the gel on top and bottom surfaces during swelling

In order for these gels to be modeled accurately, certain parameters needed to be determined. Prof. Crone and her group provided the experimental data (Young's modulus and Poisson Ratio) 
for the hydrogels. The values vary with the $\mathrm{pH}$ of the surrounding solution. However, the properties depend on a number of different aspects like the hydrogel cross-linking density and degree of exposure during polymerization. These values are also found to affect the degree of deformation considerably. Hence, exact determination of these values was important.

A simulation of the oxidation depth in a hydrogel when exposed to sodium iodate solution has also been explored to gain insights into the kinetics of hydrogel response. The aim is to predict the oxidation depth in the hydrogel for a given time of exposure and a given concentration of the outside solution. This problem was analyzed so that a suitable model can be developed.

\section{Dissolvable Gel Model}

The modeling of the swelling and dissolution of disulfide-crosslinked hydrogels (biofuse) was also completed. The dissolvable hydrogels can lead to the development of chemical and biological sensors and sacrificial structures for microfluidic systems. The mechanism of the whole process was studied in detail and suitable models for the various phases of the process was developed. After the simulation of the model showed compatibility with the experimental results, the model was used for the purpose of investigation. A variety of factors affecting the whole process were investigated using simulation. Some of these factors were, original diameter of the hydrogel, outside concentration of the thiol (Figure 3.5a), type of thiol, flow rate, crosslinking density (Figure 3.5b), and temperature (Figure 3.6a).

As this type of hydrogel is placed in a thiol solution, the thiol diffuses into the hydrogel. Then the thiolate anion attacks the disulfide bond, and a thiol disulfide interchange reaction takes place. As a result of this interchange, the disulfide bonds acting as crosslinks are cleaved. Due to the de-crosslinking, a swelling takes place during the induction period. As time progresses, more diffusion and reaction takes place. As a result the crosslinking density decreases drastically with time. After a certain time, which marks the end of the induction period, the polymer chains become linear or negligibly crosslinked. After this occurs, the linear polymer chains coming from the hydrogel starts dissolving. Depending on the type of the thiol, various situations can arise. For some thiols (e.g. DTT), the diffusion is fast while the reaction is slower. In this case the reaction is the rate limiting factor. For some thiols (e.g. TCEP at high $\mathrm{pH}$ ), both the diffusion as well as the reaction are fast. Here the dissolving time may be the rate limiting factor. While for some other thiols both diffusion and reaction may be slow. Thus depending on the type of thiol, the induction period will vary. For some cases there is no induction period.

Both the induction period and the dissolving period increases with the original diameter. Similarly the outside concentration affects both the induction period and the dissolving period. However the crosslink density is found to affect mainly the dissolving period, while the flow rate affects the dissolving period only when the bath is unstirred. When the bath has a very low flow rate then the convective mass transfer rate cannot be neglected during the dissolving period. As a result, it affects the time factor of the dissolving process. Another parameter that was exclusively investigated using simulations was the temperature. Since the temperature affects 
the reaction rate, temperature can have an important effect especially in those cases where the reaction is the rate limiting factor (e.g. DTT).

Simulations were used to investigate a case which was exactly opposite of the case for which experimental results were available in order to throw some light on an un-experimented process. A case was considered where diffusion was the rate limiting step. In fact the value of diffusivity and reaction rate were chosen in such a way that the diffusion process is much slower compared to the reaction process. The results can be explained by present understanding of the physics of the problem.

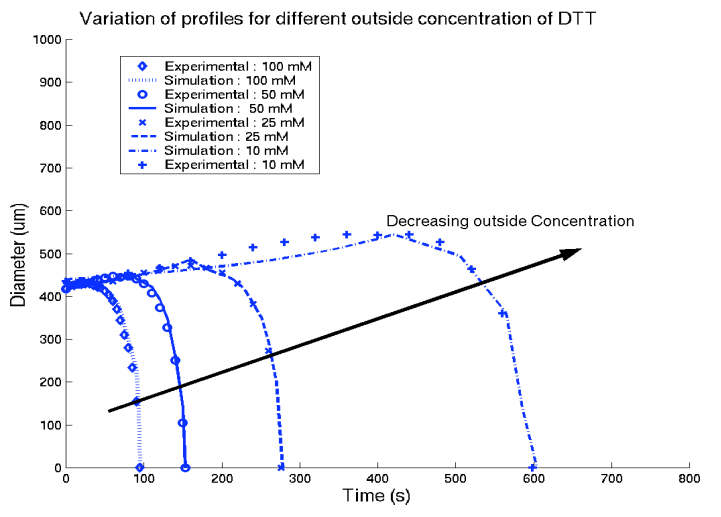

(a)

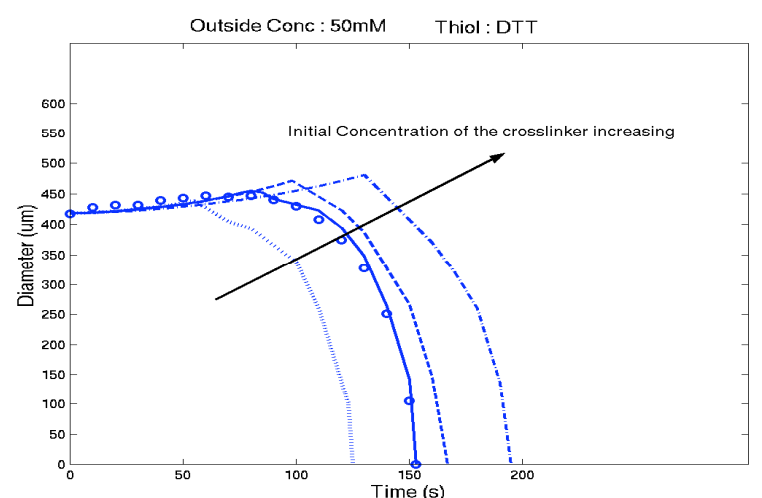

(b)

Figure 3.5. Variation of the profile of a biofuse for various outside concentration of DTT (a). and variation of the profile of a biofuse for various crosslinking density (b).

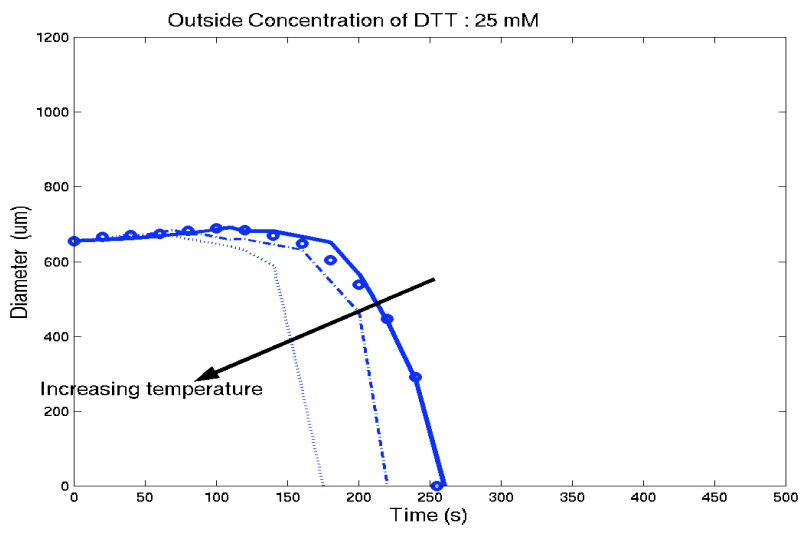

(a)

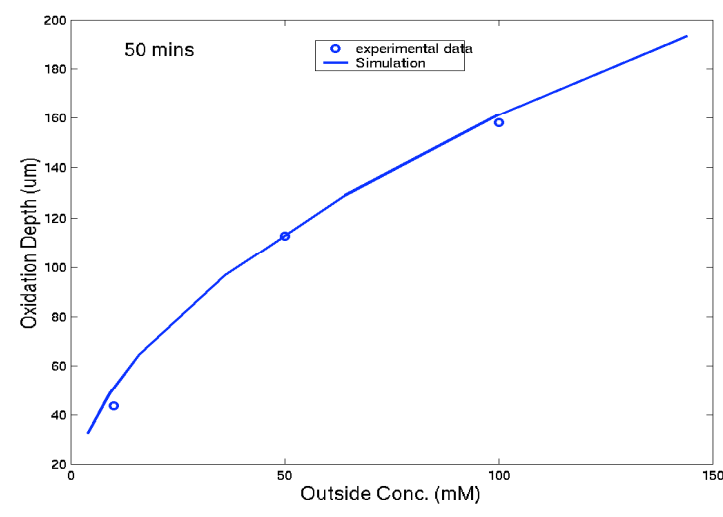

(b)

Figure 3.6. Variation of the profile of a biofuse for varying temperature (a). Variation of the oxidation depth with outside concentration is shown for a fixed time of 50 minutes $(b)$.

In another project, which involved the simulation of oxidation of diols installed in a hydrogel matrix to aldehyde, some more results were obtained that compared well with the experimental 
results. While investigating more design parameters that may affect the process, flow rate was not found to affect the process much. Presently investigations are going on using temperature as a design parameter. A model has been developed in this regard. Initial results of the simulation are encouraging [22].

Previously, models were developed for predicting the equilibrium swelling and swelling kinetics of $\mathrm{pH}$ sensitive hydrogels. While the equilibrium model worked well, the kinetic model was found to predict the swelling kinetics of larger gels accurately but not that of smaller gels. Further investigation showed that the kinetic model neglected the electrical migration term and the fluid velocity term in the Nernst-Planck equation, and it also applied the Donnan theory inside the gel. The existing model was modified by including these two terms (electrical migration and fluid velocity) in the Nernst-Planck equation. The applicability of the Donnan theory was also examined. In short, a more general model based on chemo-electro-mechanics, which solves the Poisson equation instead of applying the Donnan theory, replaced the earlier kinetic model, which was based on chemo-mechanics and the Donnan theory. Figures 3.7a and $3.7 \mathrm{~b}$ show the predictions from the new model for both larger and smaller gels. The numerical simulation matches the experimental data very well using the new model compared to the old model (Figure 3.3). The replacement of the Donnan theory by the Poisson equation and electrostatic coupling seems to play a major role in the improvement. The inclusion of the electrical migration term also improves the results slightly. The fluid velocity term has no affect in the applied range.

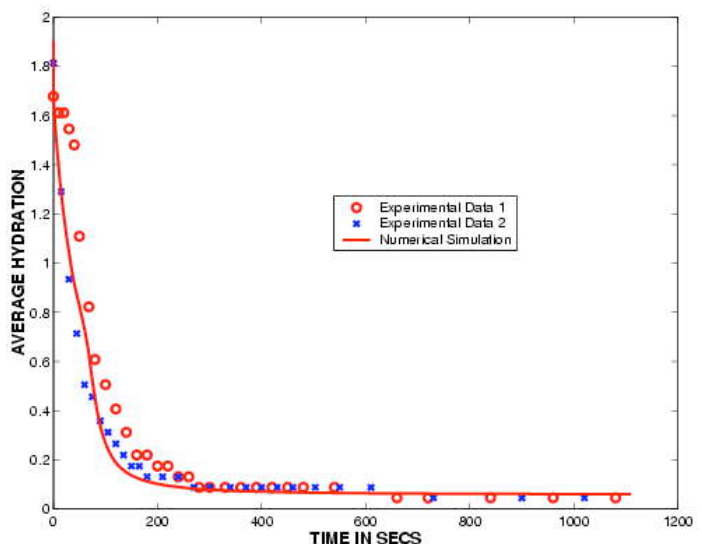

(a)

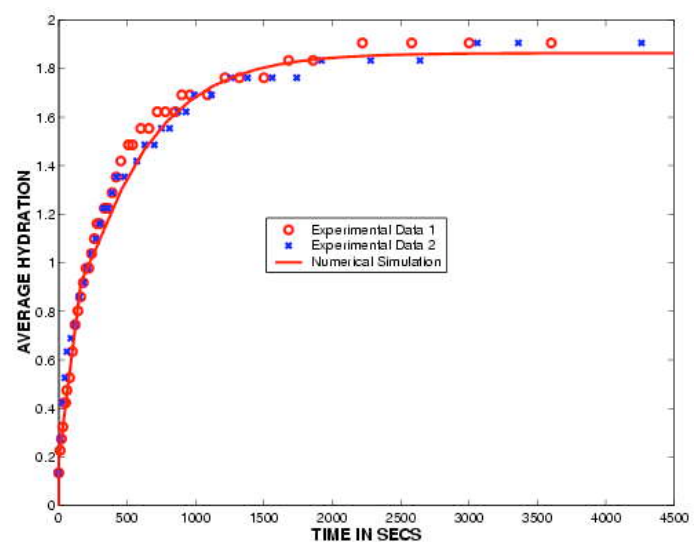

(b)

Figure 3.7. Swelling of 150-micron gel (a) and Deswelling of 150-micron gel (b) show good agreement with experimental data.

\section{Electrically Responsive Gel Model}

In addition to modeling the $\mathrm{pH}$ responsive gels, an effort was also made to model electrically responsive gels [23]. An extensive literature review on electro-active polymers and electroresponsive gels was conducted. The mechanisms behind the working of the electro-responsive gels were analyzed during the initial phase of the literature survey. The various mechanisms proposed by researchers who have worked in this field were categorized. The various models, 
which were developed for problems in the related field, were also reviewed. However, these models do not have much consensus, and a considerably new model is required for our gels. Therefore, a mathematical model for the electro-responsive gel was developed. This model incorporated the various positive aspects of the related models that are suitable for our gels. The new chemo-electro-mechanical model was used for the simulations in 2D as it already involves the electric field term in it. The electric potential was applied at the two electrodes placed in the solution, the anode was at $+10 \mathrm{~V}$ and the cathode at $0 \mathrm{~V}$, and the solution maintained at $\mathrm{pH}=10$. Swelling was observed only at the anode side of the gel and there was negligible swelling at the cathode side during the experiments. The simulations predicted this as well. Figure 8a shows the deformation profile within the gel at a particular time instant during the swelling process. The semi-circular jacket around the central semi-circular support is the gel. The left side (the anode) shows a larger deformation than the cathode side. The preliminary swelling kinetics for a electric field of $10 \mathrm{~V}$ is shown in Figure $3.8 \mathrm{~b}$.
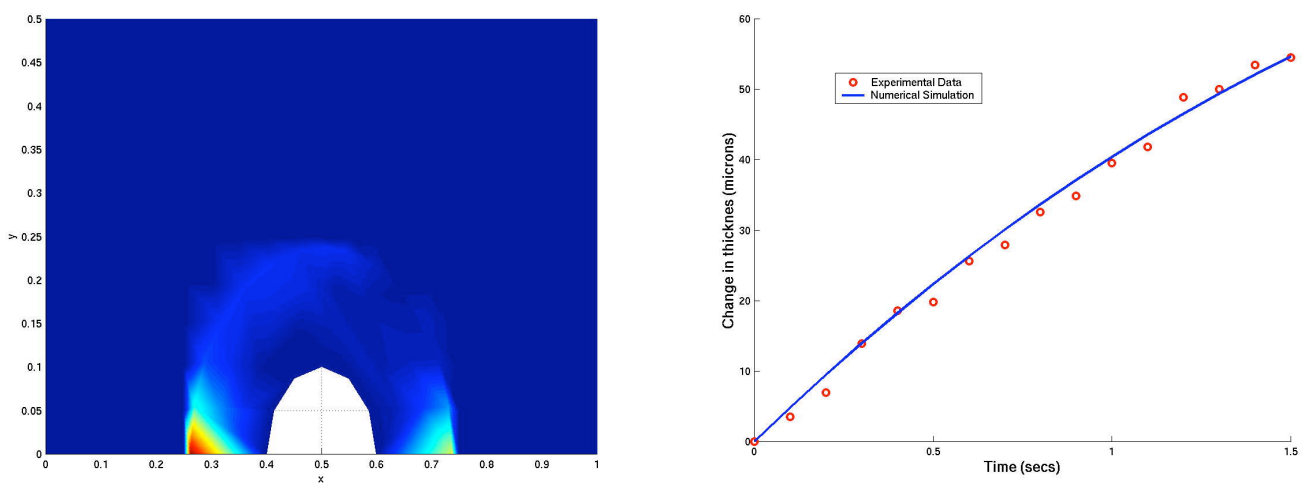

(a) (b)

Figure 3.8. Deformation profile in the gel-bath system during swelling (a) and swelling of the gel for a $10 \mathrm{~V}$ electric field (b).

In addition to force response testing, the influence of the degree of cross-linking on Young's modulus was also investigated. Seven hydrogel chemistries of $0.2,0.5,1,2,3,4$, and $5 \% \mathrm{w} / \mathrm{w}$ cross-linker were tested in tension in a buffered $\mathrm{pH} 7$ solution. Representative data is shown in Figure 3.9. 


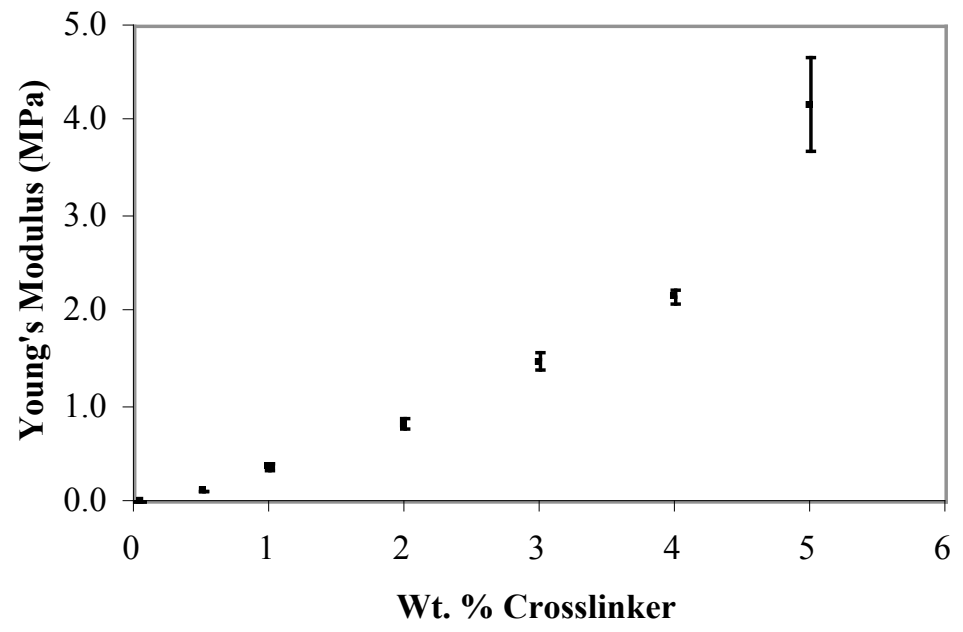

Figure 3.9. Young's modulus as a function of the $\% w / w$ of cross-linker in the hydrogel chemistry. Each data point represents the average of five samples while the error bars denote the standard deviation.

The model for predicting the behavior of hydrogels triggered by electric fields (known as Electro-responsive gels) was modified and improved. The effect of the $\mathrm{H}^{+}$ion generation at the anode and the $\mathrm{OH}^{-}$ion generation at the cathode has been taken into consideration in this model. The present model also takes into account the variation of the modulus of the gel with change in the applied electric field and with change in the hydration state of the hydrogel (Figure 3.10, 3.11). However, from the model it can be observed that the variation in the modulus of the gel is significant when the applied field is of the order of $\mathrm{KV} / \mathrm{mm}$ or $\mathrm{V} / \mu \mathrm{m}$. Simulation of the response of an E-gel was done for a number of cases. For example, the response of an electro-responsive gel with a rectangular cross section, surrounding a rectangular post, has been observed to be similar to that of an E-gel with cylindrical cross section. The response of electro-responsive gels to square voltage waveforms of differing amplitudes and duty cycles were obtained by simulation. The simulation results compared well with the experimental results. These simulations show that for constant amplitude signals, the equilibrium hydrogel volume is proportional to the duty cycle. So if the amplitude is increased, the rate of volume change (in transition between different volume cycles) is increased, as is the final equilibrium volume at higher duty cycles. The hydrogel response is found to be non-isotropic. During the application of the DC field for stimulating an E-gel, it was observed that the deswelling is taking place much slower compared to the swelling. Therefore, to get a faster deswelling an electric field was applied in the reverse direction. Initially a sharp deswelling was observed, but it was followed by a re-swelling due to reverse osmosis.

Since the size of the gel is directly related to the response, smaller gels were investigated. Nano sized gels can have a number of advantages over the micro sized hydrogels. For example, the response time of the nano gels to any stimuli will be much less than that for the corresponding micro gels. Some of the factors that were not significant for the modeling of the micro gels can become very important for the modeling of nano gel phenomenon. For example, the electrostatic 
interaction of the charged ions/particles and the charged polymer membrane (due to the presence of the fix charge) can be a very significant issue in the nano gel model.

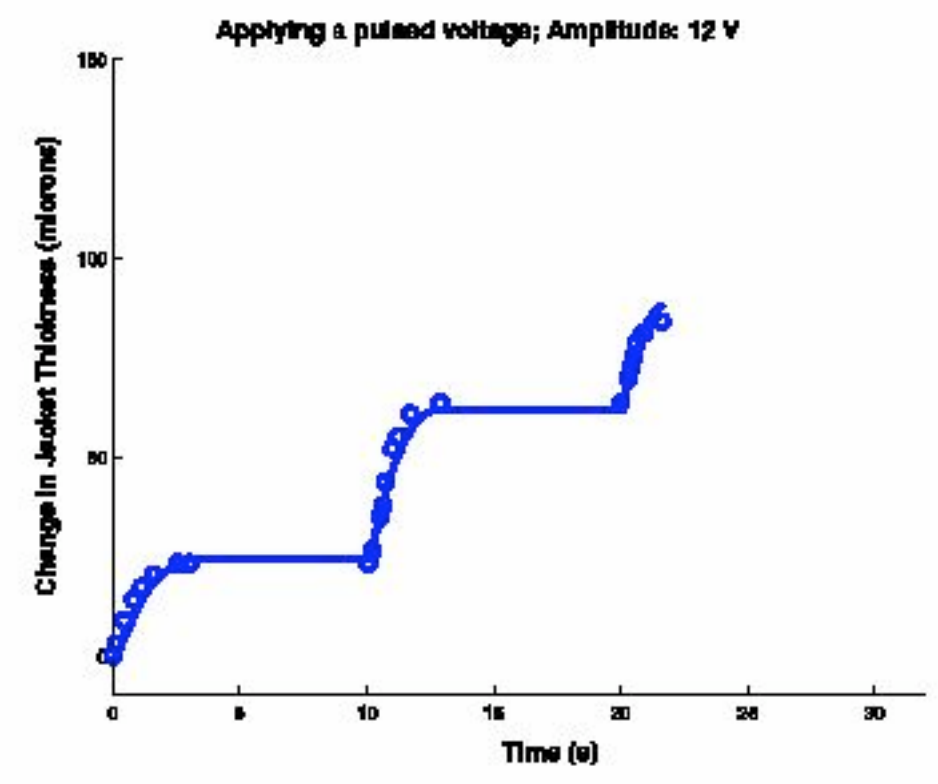

Figure 3.10. The response of an E-gel towards a voltage signal of amplitude $12 \mathrm{~V}$ with an offset of $6 \mathrm{~V}$. The duty cycle varies as $20 \%, 50 \%$ and $80 \%$.

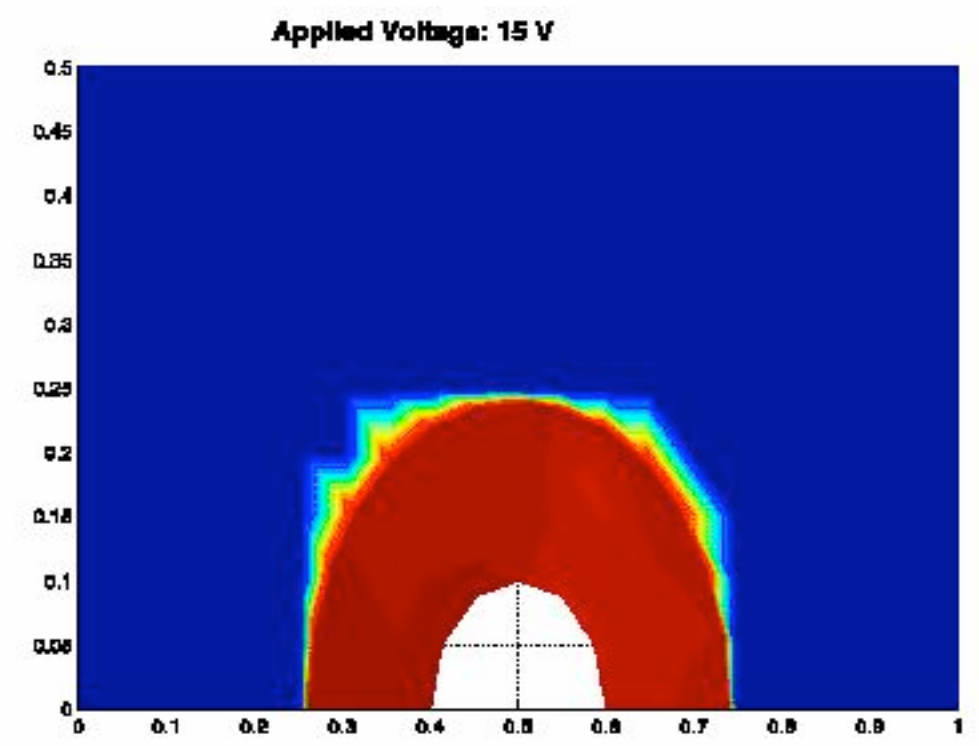

Figure 3.11. Variation in the hydration state. 
Previously, the modeling group was focused on device oriented modeling. Hydrogels have been used for a variety of applications where environmental sensitivity is needed. By controlling the functional groups along their backbone chains, hydrogels can be made sensitive to environmental conditions, such as temperature, $\mathrm{pH}$ or ionic strength. In the work done by Bashir [24] surface micromachined cantilevers were fabricated using commercially available SOI wafers with a 1 $\mu \mathrm{m}$ silicon layer and $1 \mu \mathrm{m}$ oxide layer. A $0.3 \mu \mathrm{m}$ oxide was grown on the side walls of the SOI layer, and a dry anisotropic etch was used to remove the oxide from the substrate exposed silicon surface, while leaving it on the side-walls of the SOI layer. Tetramethyl Ammonium Hydroxide, TMAH, was used to etch the silicon substrate and to release the cantilever/oxide composite structure (Figure 3.12). The device shown in Figure 3.12 can be used as an ultrasensitive $\mathrm{pH}$ microsensor. The sample can be allowed to equilibrate at a given $\mathrm{pH}$, and the deflection of the cantilever beam can be measured by adjusting the focal plane from the edge of the cantilever to the tip of the cantilever.

The physics of the bending of the composite cantilever beam was based on the mechanism of the swelling of the $\mathrm{pH}$-responsive hydrogels. The mobile ions diffuse into the hydrogel from the surrounding solution. Due to the presence of the fixed charge there is a concentration difference between the ionic concentration in the gel and in the bath, which can be predicted by the principle of Donnan equilibrium and electro-neutrality. As a result of the concentration difference, an osmotic stress develops. However, as the gel strip is exposed to the solution only in one side, while the other side of the gel strip is blocked from the solution by the silicon beam, the osmotic stress acts on one side of the composite cantilever beam. So effectively, the device is like a uniformly loaded heterogeneous beam. The following design parameters of the device are expected to play a significant role: the length of the beam, the overall thickness of the composite beam, the individual thickness of the different materials of the composite beam, the fixed charge on the back bone of the polymer chain, the initial hydration of the gel, and the salt concentration in the bath surrounding the composite beam. The model for the dynamic response of the composite beam is based on finding the concentration of the ions inside the gel and outside the gel at every time step and then finding out the osmotic stress corresponding to the difference in the ionic concentration.

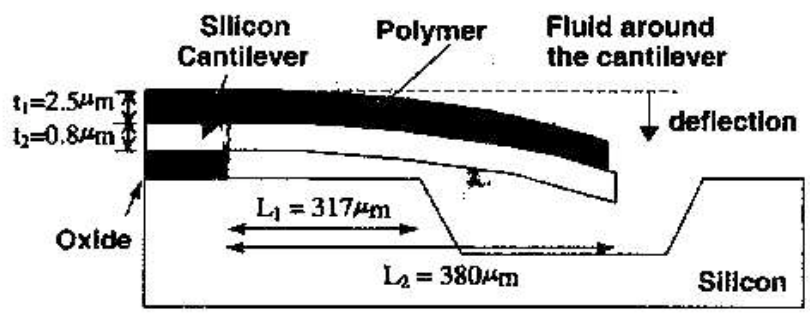

Figure 3.12. Cross-sectional schematic of the cantilever/polymer structure with the various dimensions. 
Figure 3.13 shows the comparison of the simulation results with the experimental results. It is to be noted that the tip of the beam touches the bottom surface of the cantilever well before the beam can bend fully. Thus the motion of the beam is restrained. Figure 3.14 shows the variation in the equilibrium bending of the micro-sized composite cantilever beam with varying length of the beam. Figure 3.15 shows the relation of tip deflection to the thickness of the gel only. From the results obtained, one can understand that there is a large number of design parameters to control the performance of the micro-sized composite cantilever beam. The beam can have several applications, which includes $\mathrm{pH}$ sensor and actuator based on $\mathrm{pH}$ stimulation.

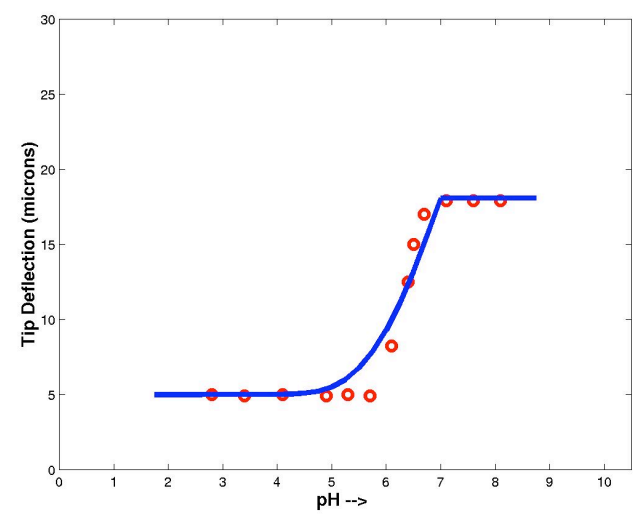

Figure 3.13. This figure shows the comparison of the simulation results with the experimental results. It is to be noted that the tip of the beam touches the bottom surface of the cantilever well before the beam can bend fully. Thus the motion of the beam is restrained. However, due to the slow response of the $\mathrm{pH}$ responsive hydrogels, hybrid actuation (simultaneous stimulus of $\mathrm{pH}$ and applied electric field) can be done to improve the response speed of the device acting as a transducer.

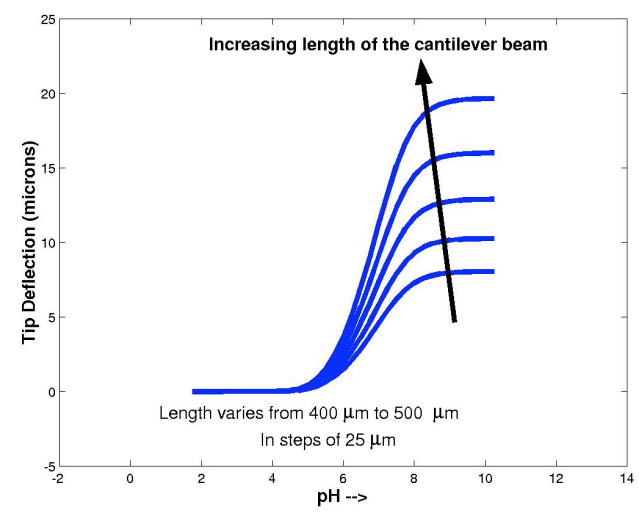

Figure 3.14. This figure shows the variation in the equilibrium bending of the micro-sized composite cantilever beam with varying length of the beam 


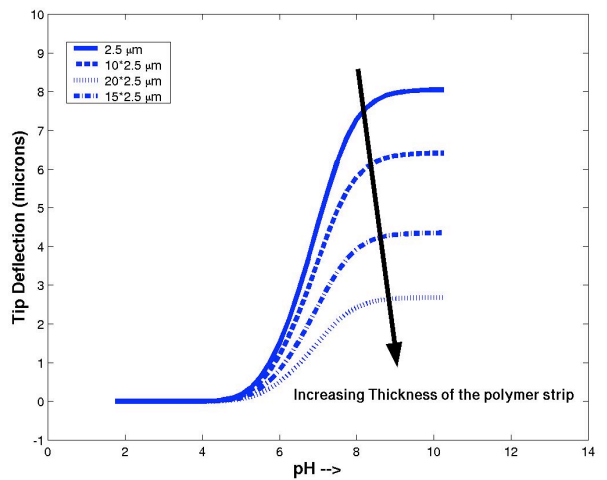

Figure 3.15. This figure shows the relation of tip deflection to the thickness of the gel only.

The effect of proper hybrid actuation on cylindrical hydrogels in a micro-channel was next considered. A significant improvement in the swelling of the $\mathrm{pH}$-responsive gels due to the presence of the applied electric field was observed (Figure 3.16). The improvement in the tip deflection of the composite cantilever beam due to the presence of hybrid actuation was also observed from simulation. The presence of field is evident in the higher $\mathrm{pH}$ range because at that range the acidic group fixed to the polymer chains dissociate and the ions from the bath solution rush into the hydrogel for minimizing the charge imbalance. Thus the presence of an external current can lead to the significant variation in the migration of ions, which in turn leads to an increased osmotic stress and an increased swelling. If the device is used as an actuator, a significantly higher actuating distance and force of actuation can be obtained by hybrid actuation compared to only $\mathrm{pH}$ stimulus (Figure 3.17). If the composite beam has to perform as a sensor then a faster response is desired. That can be obtained by simultaneous application of $\mathrm{pH}$ and electric-field stimuli. A faster response was observed by simulation of the kinetic results on hybrid stimulation. In summary, the results obtained from modeling and simulations indicate that the performance of a $\mathrm{pH}$-responsive hydrogel based transducer can be improved significantly by simultaneous application of $\mathrm{pH}$ and electric stimulation.

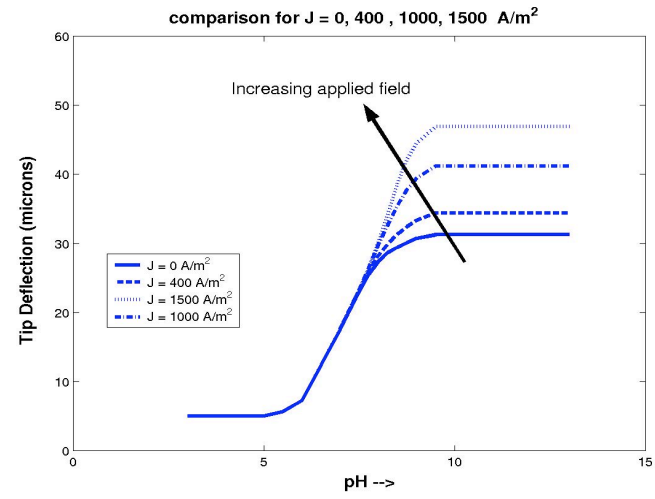

Figure 3.16. The comparison of tip deflection obtained for various hybrid actuations. 


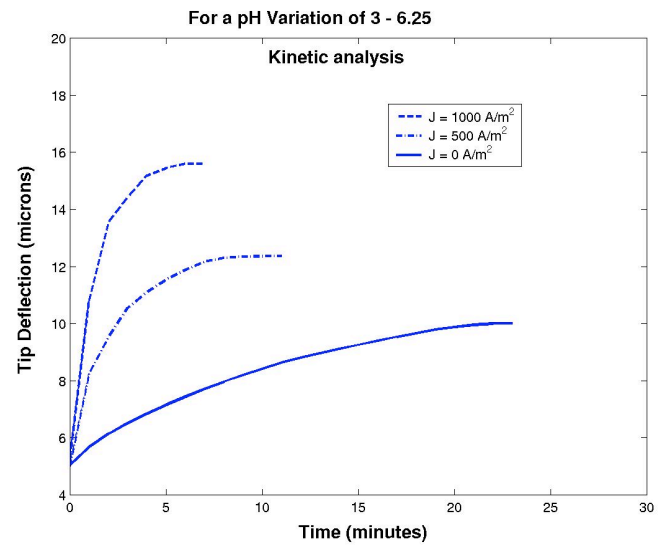

Figure 3.17. Kinetic analysis shows that the sensor can have a better speed of response in the presence of an applied field 


\section{Chapter 4 Material Characteristics}

\section{Hydrogel Properties}

Accurate modeling depends on correct mechanical behavior and material properties. The materials properties of both responsive and non-responsive materials were studied [25]. Samples for mechanical characterization of the responsive hydrogel and construction material were created with microfluidic tectonics fabrication techniques (Figure 4.1).

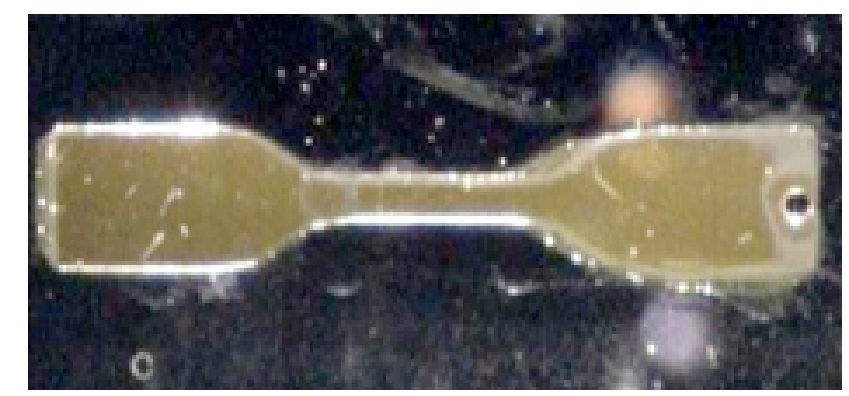

Figure 4.1 Tension sample of construction material used to determine mechanical behavior and material properties.

Standard tension samples like the one shown were used to evaluate basic mechanical behavior and determine material properties. The results of the tension tests shown in Figure 4.2 were obtained from a pH-responsive hydrogel at a displacement rate of $1 \mathrm{~mm} / \mathrm{min}, 2 \mathrm{~mm} / \mathrm{min}$, and 4 $\mathrm{mm} / \mathrm{min}$. Each sample had a $2.5 \mathrm{~mm}$ by $1 \mathrm{~mm}$ cross section and was loaded to the point of rupture. The standard dimensions of the dumbbell sample (Type IV) were scaled down due to better approximate the component sizes being developed in the BioFLIPS project. Dumbbell shaped test samples were created with the photolithographic mask technique used in microfluidic device construction. The graph shows a stress-strain relationship characteristic of a semicrystalline polymer.

Hydrogels exhibit both rubber elastic and viscoelastic properties that can be determined through traditional tensile testing and dynamic mechanical analysis, respectively. The mechanical properties of hydrogels depend on the environmental conditions in which they are tested, as well as the deformation rate at which a mechanical test is conducted. 


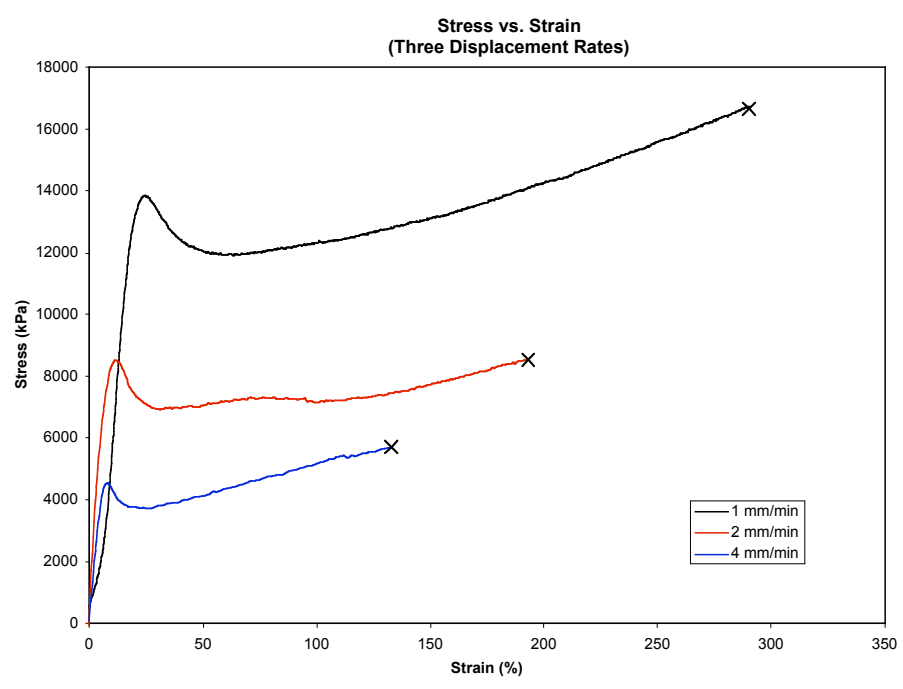

Figure 4.2. The stress-strain relationship of three hydrogel samples with displacement rates of $1 \mathrm{~mm} / \mathrm{min}, 2 \mathrm{~mm} / \mathrm{min}$ and $4 \mathrm{~mm} / \mathrm{min}$. The graph shows a stress-strain relationship characteristic of a semi-crystalline polymer.

Optical images of the dumbbell tension sample taken at intervals during the deformation process are shown for a $5 \mathrm{~mm} / \mathrm{min}$ test (Figure 4.3). These images clearly illustrate the formation and subsequent progression (cold drawing) of the neck region up to the point of fracture. The sample cross-section was $2.9-\mathrm{mm}$ by $1-\mathrm{mm}$, and its gage length was $6.25 \mathrm{~mm}$ prior to loading.

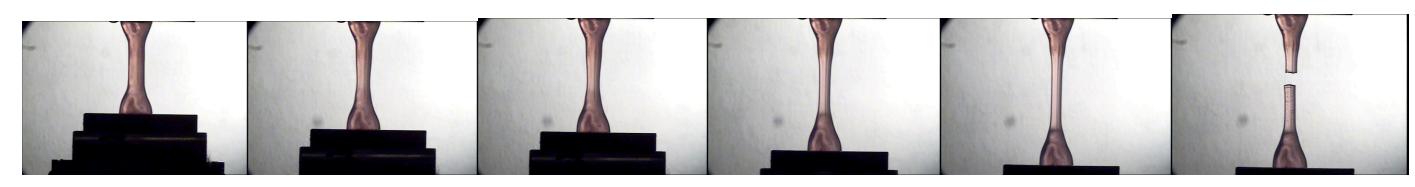

Figure 4.3. Images of the responsive hydrogel undergoing deformation.

The Young's modulus and Poisson's ratio were determined for the responsive hydrogel material, and this information was used for the modeling equations.

Material testing techniques were refined significantly through the use of the Instron Micromechanical Testing Machine. The Instron (Figure 4.4a) has better force and displacement resolution and improved sample handling. These improvements create a more uniform cross section and no observed anisotropic effects. The environmental chamber system has also been incorporated into the testing machine allowing experiments to be conducted in a fully hydrated and $\mathrm{pH}$ controlled environment. An Epon patterned silicon wafer (Figure 4.4b) was used to 
mold PDMS to form a thin stencil (Figure 4.4c). The stencil was filled with responsive hydrogel and polymerized to form the sample used in the Instron.

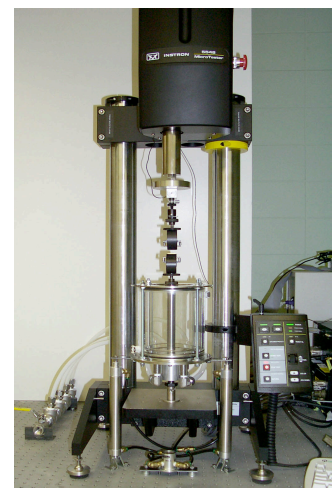

(a)

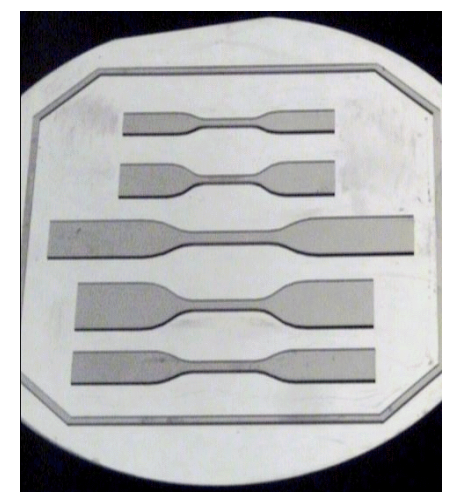

(b)

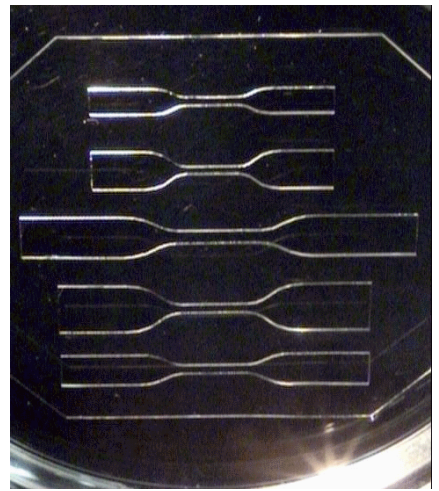

(c)

Figure 4.4. - The Instron Micromechanical Testing Machine (a). An EPON patterned silicon wafer (b) was used to mold PDMS to produce a thin stencil (c). Responsive hydrogel was injected into the stencil and polymerized to form well-defined tensile samples.

The structures created from hydrogels for use in microfluidic devices must endure forces imposed by flow of an aqueous solution, the constraint of device walls during swelling, and the restoring force of elastic membranes. Optimizing the design of these devices demands an understanding of how the mechanical properties of the gel change with its environment.

An experimental protocol was developed to determine the basic mechanical properties of the hydrogel at various levels of $\mathrm{pH}$ equilibrium. Additional tests were performed to evaluate the force produced by the hydrogel during its phase transformation. An Instron micromechanical testing machine was modified such that tensile tests could be carried out in temperature controlled $\mathrm{pH}$ solution, while optical methods of strain measurement were employed. Values of Young's modulus, Poisson's ratio, ultimate tensile strength, and percent elongation at break have been determined as a function of $\mathrm{pH}$.

Several material properties exhibit a dramatic shift at the $\mathrm{pH}$ where phase transformation occurs. For instance, the ultimate tensile strength decreases from approximately $250 \mathrm{kPa}$ to $50 \mathrm{kPa}$ with increasing $\mathrm{pH}$ (Figure 4.5), and the percent elongation at break decreases from approximately $140 \%$ to $30 \%$ with increasing $\mathrm{pH}$ (Figure 4.6). 


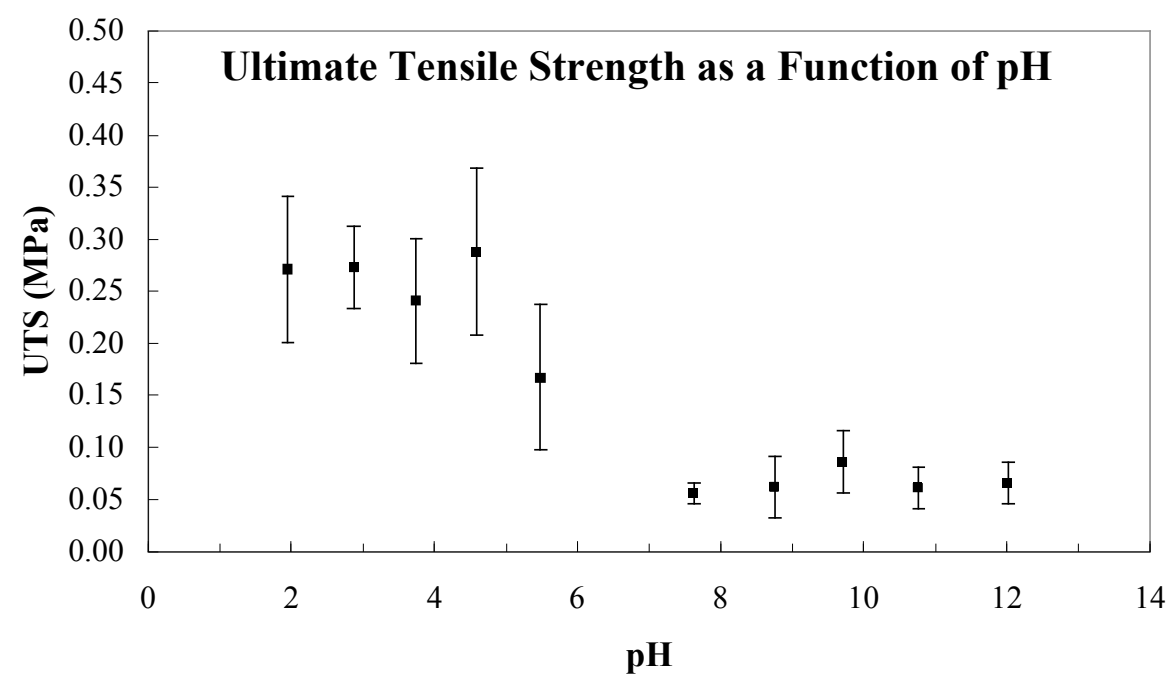

Figure 4.5. The ultimate tensile strength shows a decrease from $250 \mathrm{kPa}$ to $50 \mathrm{kPa}$ as $\mathrm{pH}$ is increased from $\mathrm{pH} 2$ to $\mathrm{pH} 12$.

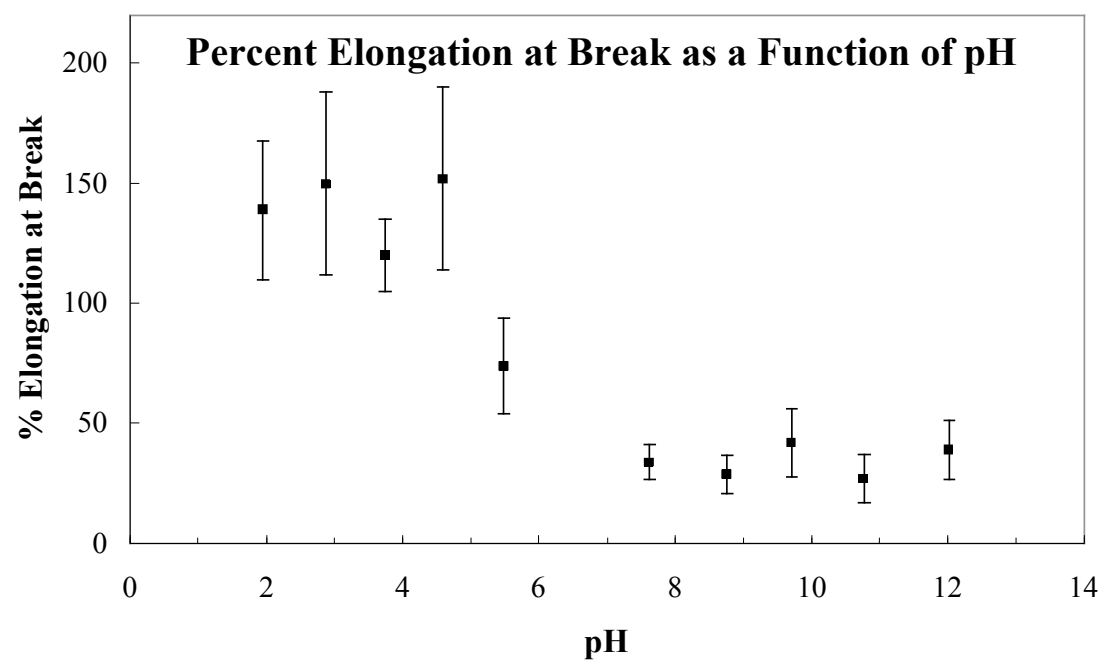

Figure 4.6. The percent elongation at break decreases from $140 \%$ at $\mathrm{pH} 2$ to $30 \%$ at $\mathrm{pH} 12$.

The interaction of water with molecules can be altered by the addition of salt. In fact, the cations and anions of neutral salts can be arranged in order of their effectiveness with respect to a particular measurement. Hofmeister found that cations and anions could be arranged according to their influence on protein solubility. The ordering arises from the effect of the salt ions on the hydrogen-bonded clusters that water forms, either with other water molecules or with polar or charged groups. Cluster formation is a function of the intensity of the electrostatic field around the ions. Smaller ions have more intense fields than large ions of the same valence, resulting in a 
greater degree of hydration. Thus, the solubility of a given protein can be extremely sensitive to both the concentration and type of salt added to the aqueous solution. This ordering is observed by many other measurements of neutral salt solutions, including surface tension at the air-water interface, the self-diffusion coefficient of water, and polymer swelling. Since a polyelectrolyte hydrogel consists of a number of fixed charge groups, it is conceivable that different ions could have different effects on the kinetics or equilibrium characteristics of polyelectrolyte hydrogels.

The effects of several concentrations of $\mathrm{NaCl}$ added to $\mathrm{pH} 2 \mathrm{HCl}$ and $\mathrm{pH} 12 \mathrm{NaOH}$ solutions are shown in Figures 4.7 and 4.8. In each case, the hydrogel was expanded and contracted by changing the $\mathrm{pH}$ between 2 and 12. The salt concentration in the initial and final solution was identical. As expected, a higher salt concentration produced a smaller degree of swelling for both the contracted and expanded hydrogel. It also appears that the different salt concentrations produced different rates of swelling.

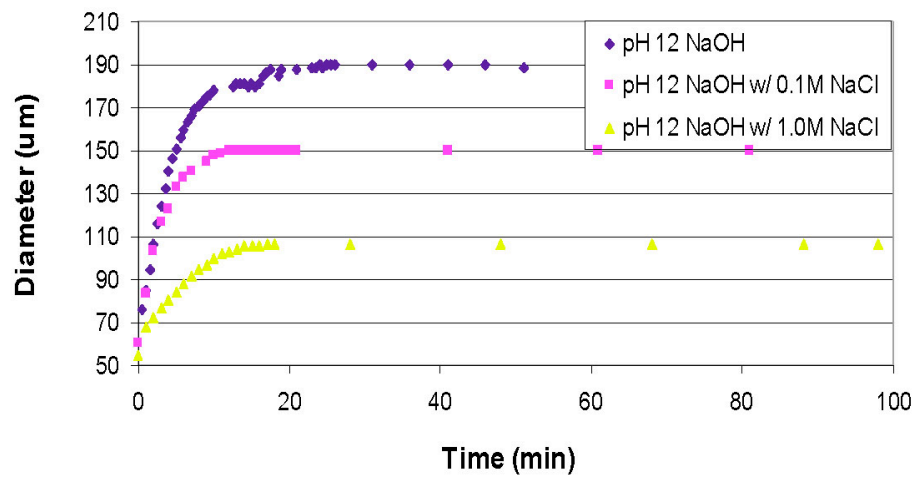

Figure 4.7. Expansion in $\mathrm{pH} 12 \mathrm{NaOH}$ solutions with various quantities of $\mathrm{NaCl}$ added.

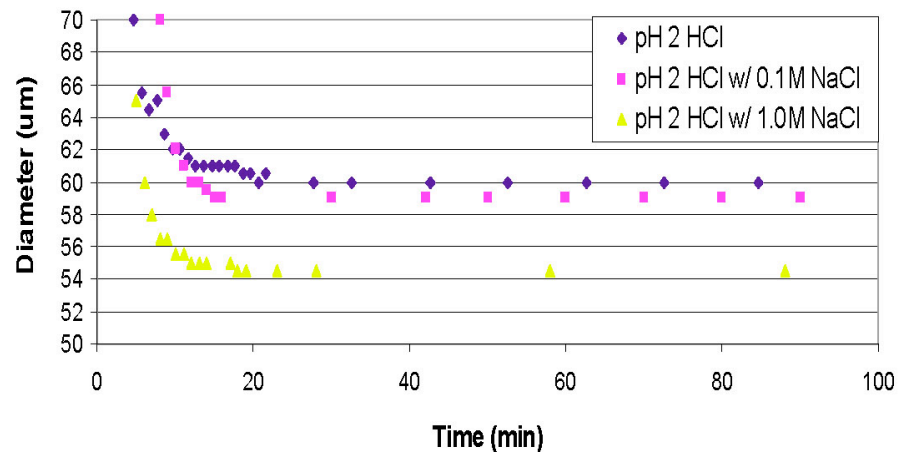

Figure 4.8. Contraction in $\mathrm{pH} 2 \mathrm{HCl}$ solutions with various quantities of $\mathrm{NaCl}$ added.

A critical parameter of interest for the design of microfluidic devices is the amount of force the hydrogel is capable of exerting during swelling. Additionally, forces acting on stationary 
channel walls must be controlled to avoid delamination and regulate frictional interactions. Thus, quantification of the magnitude and response time of the force produced by a hydrogel actuator during the swelling process was of critical importance to the effort of device optimization. The test setup was configured in a manner that would mimic the conditions of a channel in a microfluidic device. The Instron MicroTester was equipped with a $50 \mathrm{~mm}$ diameter stainless steel compression platen that supported a shallow glass dish filled with buffered $\mathrm{pH}$ solution. A custom-built acrylic compression platen $(6.35 \mathrm{~mm}$ diameter) was connected to a 10 $\mathrm{N}$ load cell and served as an analogue to the upper wall of a microchannel.

Figure 4.9 shows a side view of a typical test specimen. The sample is dried and kept in air for storage prior to testing. For testing, the hydrogel was placed in the glass dish and the upper compression platen was manually lowered onto the top surface of the surrounding PDMS sidewalls. After a buffered $\mathrm{pH}$ solution was poured into the glass dish, the load cell was reset to account for buoyancy effects on the platen. Load data was acquired over time while holding the displacement constant. A typical force response curve for a $600 \mu \mathrm{m}$ diameter sample is shown in Figure 4.10. A hydrogel of this size is capable of exerting $0.14 \mathrm{~N}$ on the upper platen of the test machine after approximately 2.1 hours of swelling.

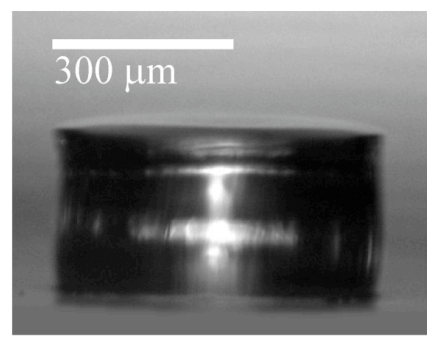

Figure 4.9. Optical image (side view) of a hydrogel used in force response tests. The cylinder has an approximate diameter of $600 \mu \mathrm{m}$ and height of $300 \mu \mathrm{m}$.

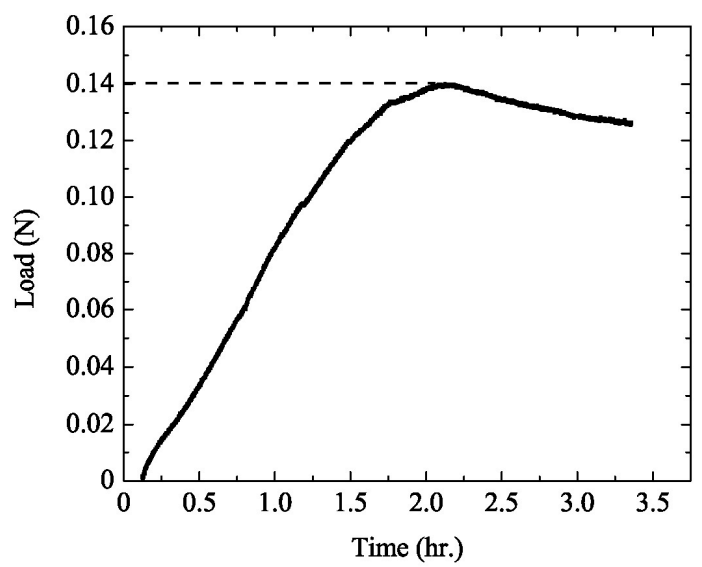

Figure 4.10. Typical force response curve generated by a 600 um hydrogel structure swelling in pH 12 buffer. The hydrogel exerted a maximum force of $0.14 \mathrm{~N}$ on the upper platen of the test machine after approximately 2.1 hours of swelling. 
In addition to force response testing, the influence of the degree of cross-linking on Young's modulus was investigated. Seven hydrogel chemistries of $0.2,0.5,1,2,3,4$, and $5 \% \mathrm{w} / \mathrm{w}$ crosslinker were tested in tension in a buffered $\mathrm{pH} 7$ solution. Representative data is shown in Figure 4.11 .

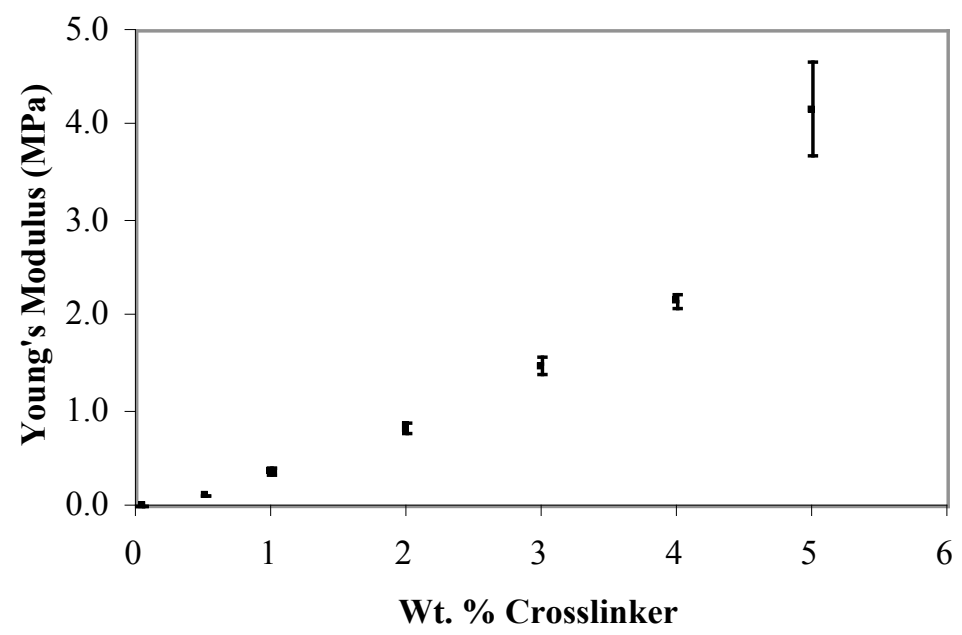

Figure 4.11. Young's modulus as a function of the $\% w / w$ of cross-linker in the hydrogel chemistry. Each data point represents the average of five samples while the error bars denote the standard deviation.

Although the force of a hydrogel cylinder was measured, an array of hydrogel posts was also tested. A sample with forty hydrogel cylinders, arranged similar to the ones in the hydrogel actuated pump device, was designed and tested. This sample design can be directly compared to the behavior of devices and eliminates inconsistencies that arise due to alignment of an individual cylinder sample. A series of pictures showing the swelling of part of the array is in Figure 4.12. This test was carried out to verify that the hydrogels were not coming in contact with each other while approaching the swollen state.

The force response of a hydrogel is very sensitive to a number of different parameters that arise during fabrication and testing. Precise and repeatable sample geometry is essential. The sample system is designed so that a channel configuration is mimicked. Sidewalls are made of glass so that the test machine can use the sidewalls as a displacement stop. The test platen creates the top of the channel, and $\mathrm{pH}$ solution flows through the channel from a circulating bath. Because the cover glass had slight variations in thickness, the samples created also showed slight variation in the cylinder heights (about 5 microns). With small changes in the hydrogel volume the maximum force response dramatically changed. The dependence of load on the volume can be seen in Figure 4.13. 


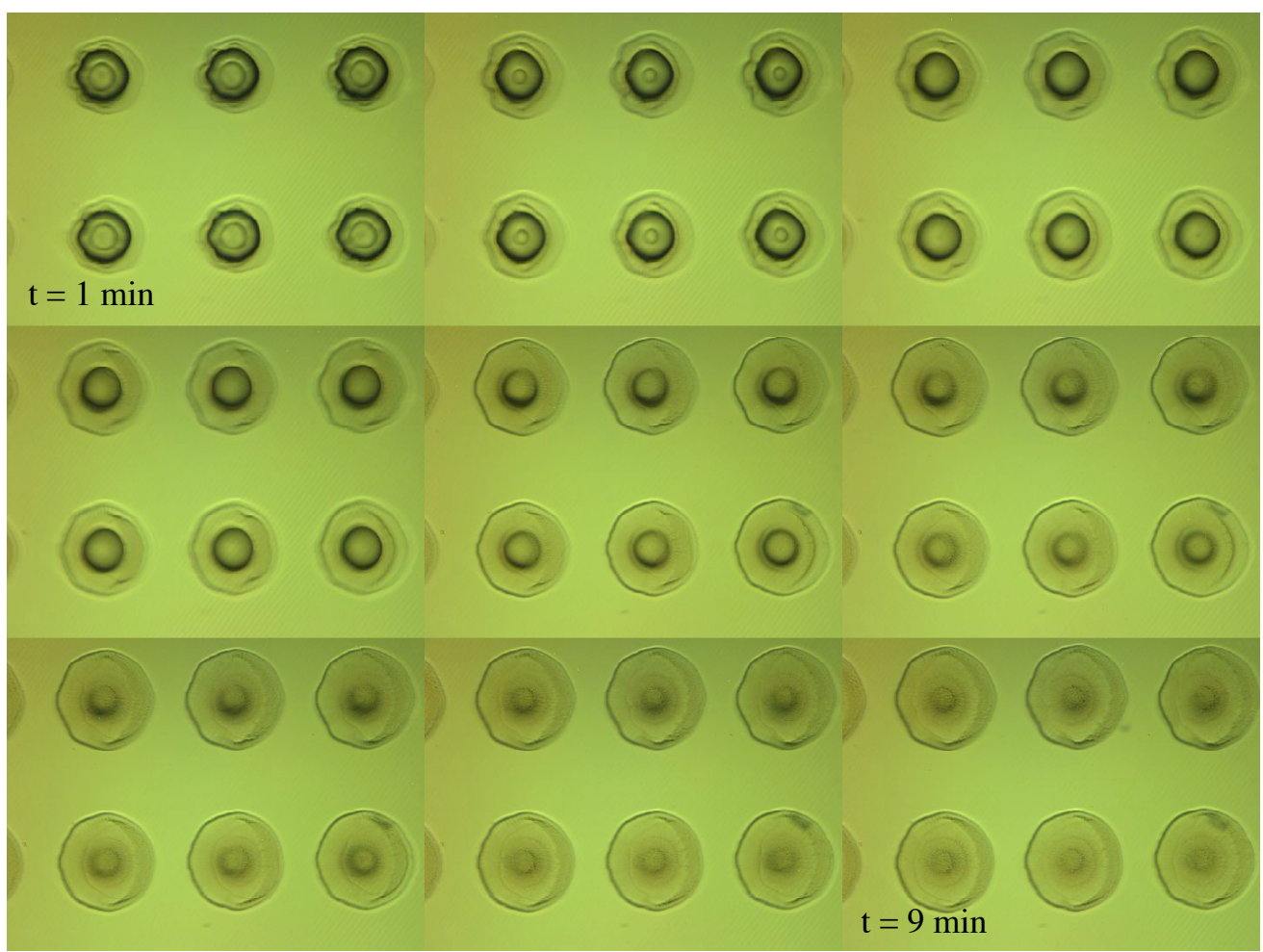

Figure 4.12. Series of time lapsed (1 min intervals) pictures of a partial hydrogel array after being exposed to $\mathrm{pH} 12$.

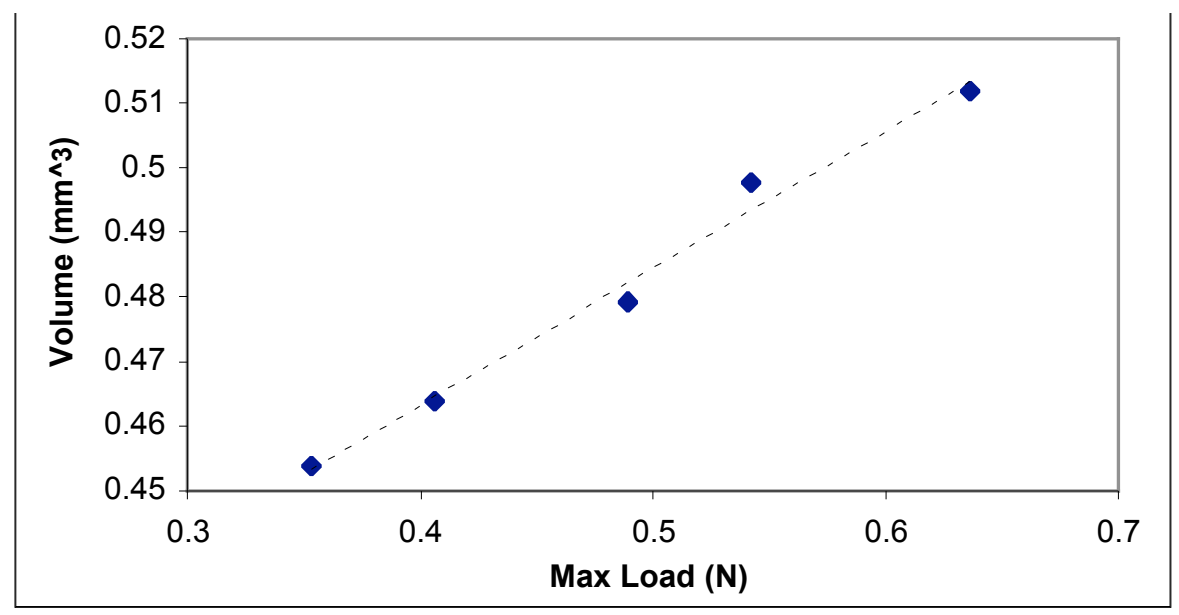

Figure 4.13. The maximum load exerted by an array of 40 gels plotted versus their respective volumes. 
Experiments were conducted to assess the effects of varying HEMA:AA molar ratios on the Young's modulus of hydrogels (Figures 4.14 and 4.15). These experiments are an extension of the previous experiments that characterized the change in mechanical properties due to variation of crosslinker over a wide range of levels. The specimens tested were dog-bone samples created in PDMS molds and polymerized with UV light. Samples were exposed to pH 7 buffer at least 24 hours prior to and during testing. Tensile tests were performed on an Instron 5548 Microtester at an extension rate of $5 \mathrm{~mm} / \mathrm{min}$. Modulus data was calculated from the linear portion of the stress/strain curve.

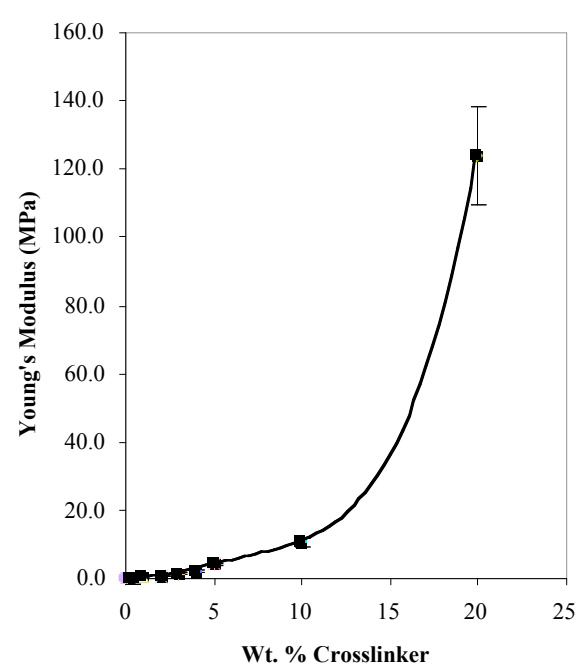

(a)

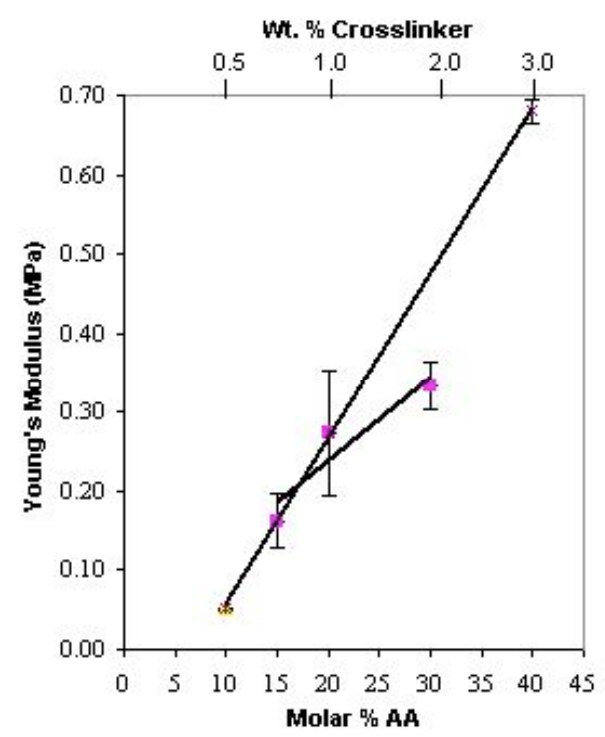

(b)

Figure 4.14. The variation of Young's modulus with weight percent crosslinker is shown (a). The standard hydrogel chemistry used in the BioFLIPS designs incorporates $1 \%$ crosslinker. Each data point represents 5 samples. (b) The impact of both crosslinker variation and acrylic acid variation are shown for values near the standard hydrogel chemistry $(1 \mathrm{wt} . \%$ crosslinker, 20 molar\% AA). 


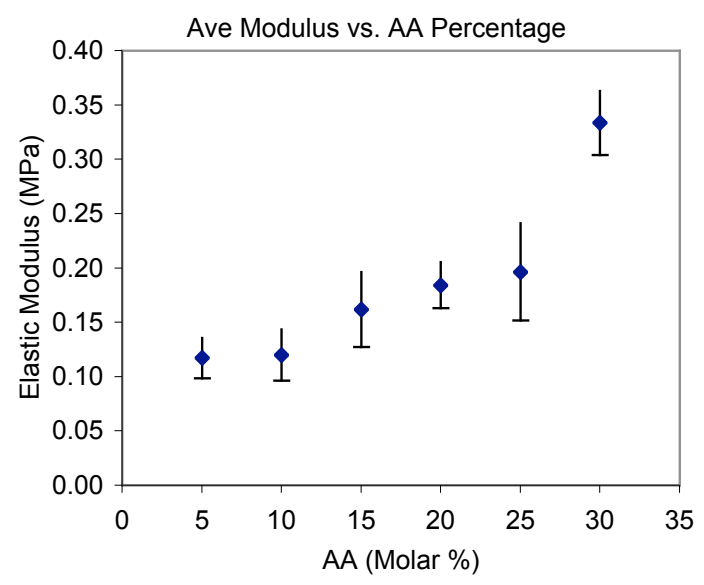

Figure 4.15. The impact of molar percent acrylic acid on the elastic modulus of standard hydrogel chemistry.

Optimizing the design of these microfluidic devices with hydrogel components demands an understanding of how the mechanical properties of the gel change with its surrounding solution. To assist device designers, material properties must be determined in order to ensure functional and reliable structures. Our research has demonstrated that the mechanical properties of HEMAAA hydrogels are strongly dependent on the degree of equilibrium swelling [26]. Adjusting the crosslinker component of the hydrogel is an effective method of controlling mechanical properties such as modulus, while the acrylic acid content effectively controls the gel swelling (Table 4.1). With precise control over mechanical and swelling characteristics, microfluidic device designers will have the knowledge required to produce optimal hydrogel valves and sensors.

The force response of a hydrogel is pressure that is exerted by a gel due to its swelling in a constrained environment. A new in-situ fabrication process was created for the force response samples. A system (Figure 4.16) was built directly on the testing platform where the samples can be made and tested. This new procedure helped to eliminate many of the variables contributing to the variation in prior test results. In addition, the new procedure better mimicked the conditions in a microfluidic device.

Since Polydimethylsiloxane (PDMS) was used in some components, certain mechanical properties were of interest. PDMS tensile specimens were fabricated and tested in accordance with ASTM standard D638-99 with an Instron 5548 MicroTensile Tester (Figure 4.17). The study quantified the stiffness of (PDMS) as a function of curing parameters, weight ratio of prepolymer to hardener, and aging. The experiments reveal the manufacturer's recommended curing parameters yield devices with different material properties. Since PDMS is often used as an engineering material (Figure 4.18), the understanding of how the stiffness changes as a result of curing parameters is an important issue. 


\begin{tabular}{|c|c|c|c|c|c|c|c|}
\hline \multicolumn{2}{|c|}{$\begin{array}{l}\text { \% Change in } \\
\text { Diameter } \\
\text { E = Elastic } \\
\text { Modulus with } \\
\text { S = Standard } \\
\text { Deviation }\end{array}$} & \multicolumn{6}{|c|}{ Acrylic Acid Content (\% Molar in monomer mixture) } \\
\hline \multirow{11}{*}{ 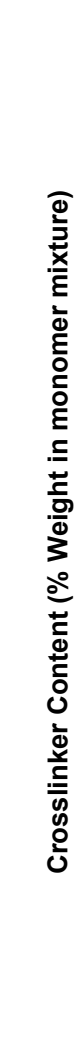 } & & 5 & 10 & 15 & 20 & 25 & 30 \\
\hline & 0.2 & & & & $\begin{array}{c}E=.034 \mathrm{MPa} \\
\mathrm{S}=.004\end{array}$ & & \\
\hline & 0.5 & & & & $\begin{array}{c}E=.11 \mathrm{MPa} \\
\mathrm{S}=.01\end{array}$ & & \\
\hline & & $160 \%$ & $190 \%$ & $200 \%$ & $210 \%$ & $230 \%$ & $240 \%$ \\
\hline & 1 & $\begin{array}{c}\mathrm{E}=.117 \mathrm{MPa} \\
\mathrm{S}=.018\end{array}$ & $\begin{array}{c}\mathrm{E}=.120 \mathrm{MPa} \\
\mathrm{S}=.024\end{array}$ & $\begin{array}{c}\mathrm{E}=.162 \mathrm{MPa} \\
\mathrm{S}=.035\end{array}$ & $\begin{array}{c}\mathrm{E}=.184 \mathrm{MPa} \\
\mathrm{S}=.021\end{array}$ & $\begin{array}{c}\mathrm{E}=.197 \mathrm{MPa} \\
\mathrm{S}=.045\end{array}$ & $\begin{array}{c}\mathrm{E}=.334 \mathrm{MPa} \\
\mathrm{S}=.030\end{array}$ \\
\hline & 2 & & & & $\begin{array}{c}E=.83 \mathrm{MPa} \\
\mathrm{S}=.05\end{array}$ & & \\
\hline & 3 & & & & $\begin{array}{c}E=1.48 \mathrm{MPa} \\
\mathrm{S}=.1\end{array}$ & & \\
\hline & 4 & & & & $\begin{array}{c}E=2.16 \mathrm{MPa} \\
\mathrm{S}=.08\end{array}$ & & \\
\hline & 5 & & & & $\begin{array}{c}\mathrm{E}=4.17 \mathrm{MPa} \\
\mathrm{S}=.48\end{array}$ & & \\
\hline & 10 & & & & $\begin{array}{c}E=10.7 \mathrm{MPa} \\
\mathrm{S}=1.09\end{array}$ & & \\
\hline & 20 & & & & $\begin{array}{c}E=124 \mathrm{MPa} \\
\mathrm{S}=14.5\end{array}$ & & \\
\hline
\end{tabular}

Table 4.1. Values for swelling (percent change in diameter) and mechanical stiffness (elastic modulus) are given for an array of gel compositions. Both acrylic acid content and crosslinker content are varied. 


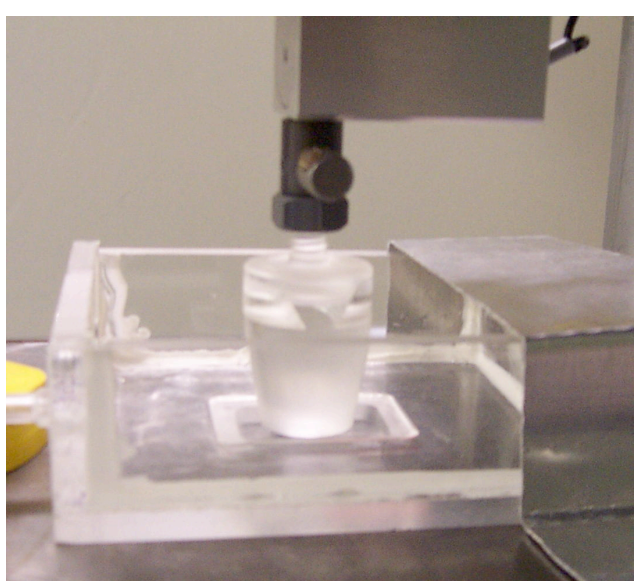

(a)

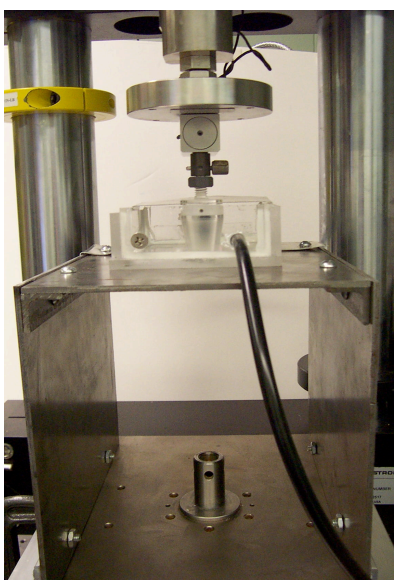

(b)

Figure 4.16. Side view of sample chamber (a) and front view of sample chamber and loading platform (b) designed for force response testing. The platen attached to the load cell at the top of the figure is held at a fixed displacement. The cylindrical hydrogel sample is polymerized in situ using UV irradiation from below. Solutions of controlled $\mathrm{pH}$ level are circulated in the chamber during testing.

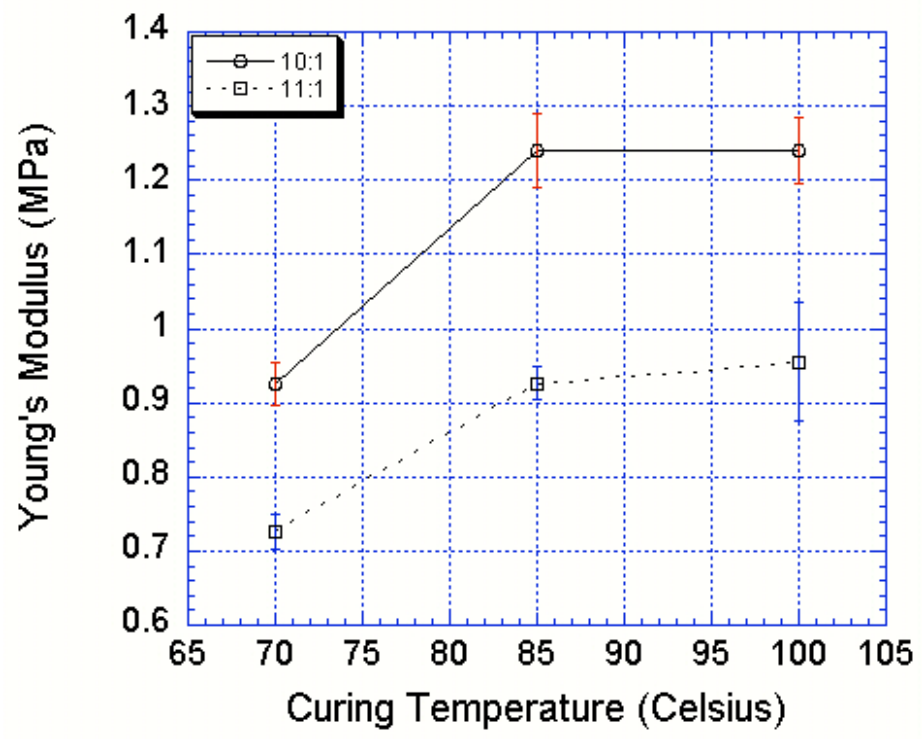

Figure 4.17. Graph of the Young's Modulus with respect to curing temperature for PDMS with a 10:1 and 11:1 weight ratio of prepolymer to hardener. 
a.

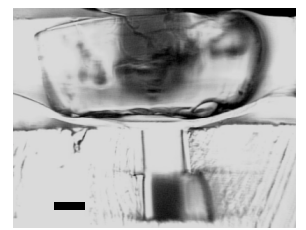

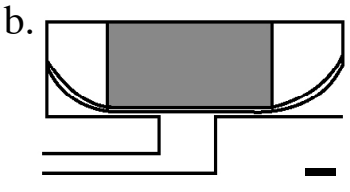

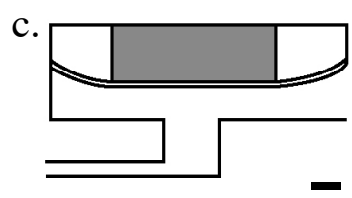

Figure 4.18. Affect of membrane stiffness on PDMS membrane valve operation. Cross section image (a) and schematic (b) of a hydrogel membrane valve cured at $70^{\circ} \mathrm{C} .(\mathrm{c})$ Schematic cross section of a membrane valve cured at $85^{\circ} \mathrm{C}$. The effects of aging may cause a valve to function normally initially, but fail due to shelf life issues later. The scale bar corresponds to $100 \mu m$.

\section{Particle Image Velocimetry (PIV)}

The patterning of surface free energies inside microfluidic networks can be used to direct fluid flow. In order to understand how the fluid was interacting with the surface in this environment, PIV was performed [27]. One method of patterning the surface energies involves pumping chemicals into a microchannel such as in Figure 4.19a, creating permanent hydrophobic pathways on otherwise hydrophilic surfaces. These pathways will then confine fluids introduced into the channel to the hydrophilic regions, if the hydrophilic surfaces are sufficiently close to one another and the driving pressure is below a critical value (Figure 4.19b).

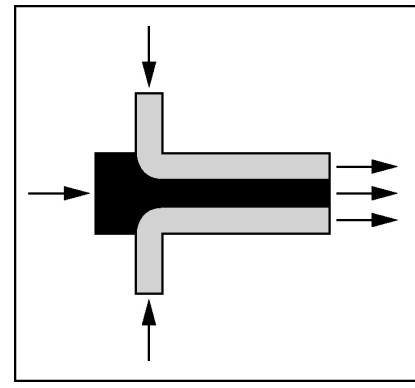

(a)

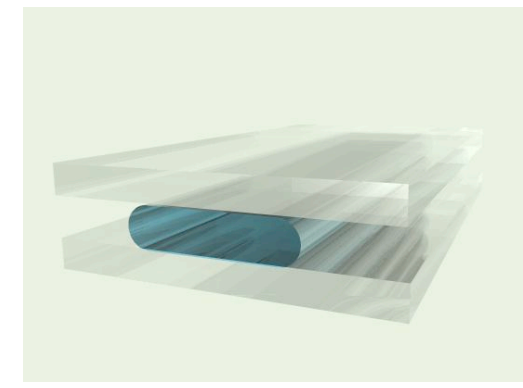

(b)

Figure 4.19. Schematic showing the laminar flow of chemicals that are used to define the hydrophobic and hydrophilic regions of the channel. The gray regions become hydrophobic after the chemicals are removed $(a)$. A schematic three-dimensional representation of the fluid flow in the channel (b).

A particle image velocimetry (PIV) analysis was conducted on three devices fabricated in this manner. PIV allows the calculation of local flow velocities by tracking the motion of neutrally buoyant particles as they are transported by the flow. Images of the (fluorescent) particles taken with a microscope/CCD camera setup are then analyzed to yield a grid of velocity vectors, where each velocity vector represents the average flow velocity in the "interrogation spot" surrounding the vector. For flows with no out-of-plane component, a three-dimensional reconstruction of the 
flow field was created by repeating this procedure at various focal planes. The thickness of the focal plane is a function of the microscope optics, and for the results presented here, the focal plane is $10.5 \mu \mathrm{m}$ thick.

Figures $4.20 \mathrm{a}, \mathrm{b}$ show the results of a PIV analysis on the flow of deionized water in a hydrophilic pathway that is approximately $165 \mu \mathrm{m}$ tall and $625 \mu \mathrm{m}$ wide. The flow was driven by a constant pressure head reservoir (gravity) to avoid any perturbations that might be introduced with mechanical pumps. The dimensions of the reservoir were sufficient to ensure that over the course of the experiment, the driving pressure varied by less than one percent. One micron diameter particles added to the reservoirs allowed the PIV analysis to be performed. Each calculated vector represents the flow velocity in a rectangular volume that is $6.37 \%$ of the total pathway depth and $4.5 \%$ of the width. The calculated vectors at each $\mathrm{z}$ position were then averaged to generate the curves shown in Figure 4.20a. For the seven focal planes investigated, the centerline velocity is plotted versus the y position in Figure 4.20b. From these figures, it can be seen that the flow in the $x-y$ direction (between the hydrophilic surfaces) follows a parabolic velocity distribution, while the flow in the $\mathrm{x}-\mathrm{z}$ direction is non-zero at the air-water boundary and relatively constant in the interior.

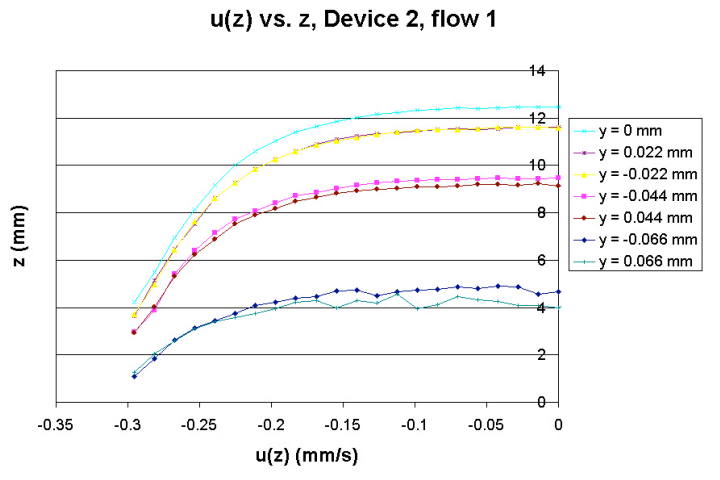

(a)

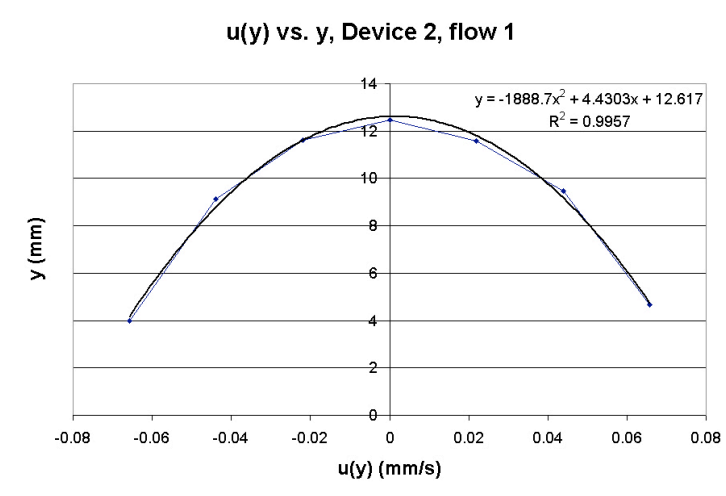

(b)

Figure 4.20. Velocity profiles (a) in the $x$-z plane are shown (looking down at the top of the channel as shown in Figure 4.19b). The curves are the velocity profiles at various depths (y positions). Reconstruction of the flow $(b)$ in the $x-y$ plane from the centerline velocities of the curves as shown in (a).

Although unable to be resolved at this resolution, it appears that a recirculation region has developed where the air-water interface connects to the device input and output connections. An image of the device input connection is shown in Figure 4.21. Also, optical observations indicate that some of the particles (in a region $5-10 \mu \mathrm{m}$ from the air-water interface) are flowing against the bulk flow. Confirming these flow characteristics and their relationship constitutes future work, and will require further studies with higher magnification and smaller particles. 


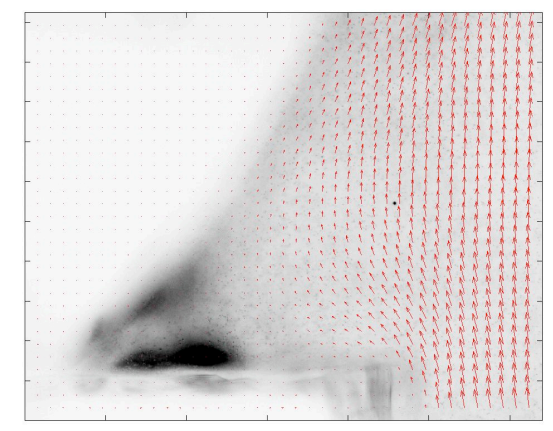

Figure 4.21. Calculated velocity vectors superimposed on an image of the fluorescent particles moving near the channel input. The image has been altered (black regions converted to white, and vice versa) to more clearly show the air-water interface.

\section{Electric Field Responsive Hydrogels}

Hydrogels that change volume under the influence of an electric field have been demonstrated previously, but most, if not all experiments, have been carried out at scales much larger than is of interest for microfluidic applications. Determining the characteristics of these hydrogels at the microscale and adapting their functionality for use in the $\mu$ Fluidic Tectonics platform was investigated [23].

Initial experiments involved placing thin silver wires in a microchannel and fabricating hydrogels between them. The gels used here were the $\mathrm{pH}$ responsive hydrogels investigate previously using chemicals. When a small bias was applied (between 1 and 10 volts), asymmetric swelling of the hydrogel was observed within seconds and continued until parts of the hydrogel were swollen many times the original volume. Figure 4.22 shows an example of these first-generation experiments.
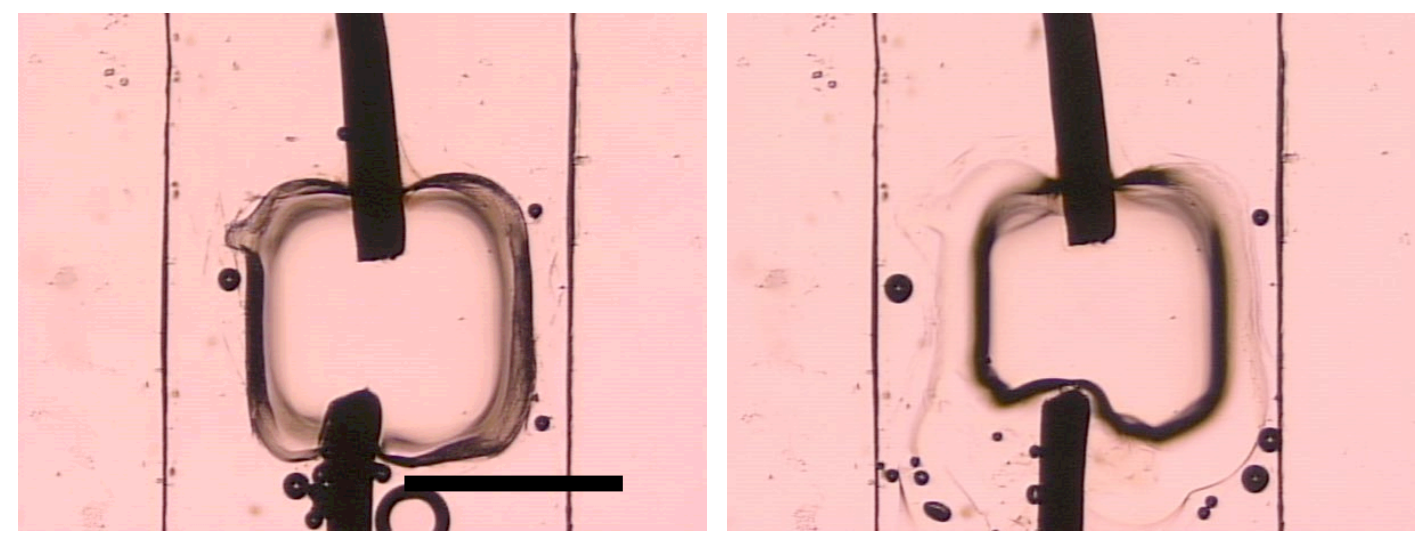

Figure 4.22. A pH-sensitive hydrogel was fabricated between two silver wires. The first image is of the water-swollen hydrogel before any voltage was applied. The second image shows the hydrogel 5 minutes after a $3 \mathrm{~V}$ potential was introduced. The scale bar is approximately $1 \mathrm{~mm}$. 
Current trials use gold electrodes patterned on a glass substrate overlaid with PDMS channels. This setup will allow smaller hydrogels to be fabricated, which will improve the time response of the swelling. Also, hydrogels that are not pierced by large electrodes will have smoother features and will not be as likely to break apart, as was observed in the initial experiments. Another advantage is that the shape and size of patterned electrodes can be modified to optimize the delivery of the electric field for the best response.

The physical mechanisms for the electrical triggering of hydrogels are not well understood. Some experimenters believe that the electrochemical reactions taking place at the electrodes are responsible for changing the composition of the fluid medium and thereby causing a change in the hydrogel. Others maintain that the electric field induces a migration of free ions in the hydrogel interior, and these ions transport water in or out of the gel. Initial experiments have been performed using gold electrodes patterned on a quartz substrate, with channels and hydrogels fabricated on top. This setup provides great flexibility as to the geometry and spacing of the electrodes and hydrogels, as well as providing for a microfluidic environment that can be closely controlled and easily viewed. Figure 4.23 shows this prototype.
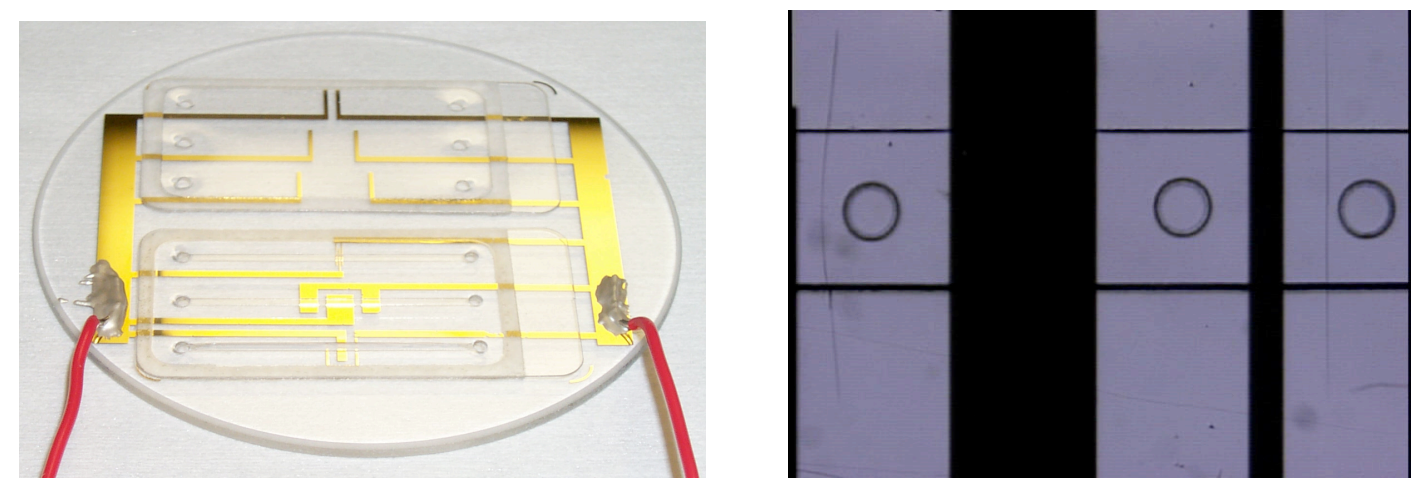

Figure 4.23. The prototype on the left utilizes the cartridge fabrication process to create microfluidic channels. The image on the right shows hydrogels as they appear in the finished device.

These experiments provided some interesting results. First, it appears that only hydrogels in between the electrodes show a response. This points to a direct link between the electric field and hydrogel response. Also, it has been observed that there is a relationship between the flow rate of the fluid medium in the channel and hydrogel response. What this implies is that the electrochemical reaction products are having an effect on the hydrogel. It seems, then, that both proposed mechanisms might be at work. It is possible that flow in the channel serves to carry away any electrochemical reaction products, and that at different flow rates there is a change in the relative dominance of the two proposed mechanisms. This would be a significant finding, and it should be pointed out that none of the previous studies on electrically responsive hydrogels consider fluid flow as a factor. 
Though it is of importance to clarify the mechanism for electrical response, the primary thrust of this line of experiments is to develop a predictable and robust actuator for use in the $\mu$ Fluidic Tectonics platform. By using materials (UV patterned hydrogels and construction materials) and processes (cartridge fabrication process) that are already a part of the platform, it is hoped that once characterization is finished there will be few additional adjustments needed to integrate electrically-triggered hydrogels into microfluidic systems. Currently modeling studies will also be useful in understanding the electrical response of the hydrogels.

Here we present further results showing that applying non-DC voltage waveforms with varying pulse widths to the hydrogel allows precise control of hydrogel volume. Figure 4.24 shows hydrogel jacket thickness as a function of time for three different square wave voltage signals. At time $=0$ seconds, a $50 \mathrm{~Hz}$ signal with duty cycle of $20 \%$ is applied to the electrodes. At 10 seconds, it is raised to $50 \%$, at 20 seconds, $80 \%$, and at 30 seconds the voltage is turned off. These experiments show that, for constant amplitude signals, the equilibrium hydrogel volume is proportional to the duty cycle. As amplitude is increased, the speed of volume change (in transition between different duty cycles) is increased, as is the final equilibrium volume at higher duty cycles. The hydrogel response is non-isotropic (Figure 4.25). Volume change is first observed nearest the anode (where jacket thickness is reported in Figure 4.24) and then in areas further away from the anode.

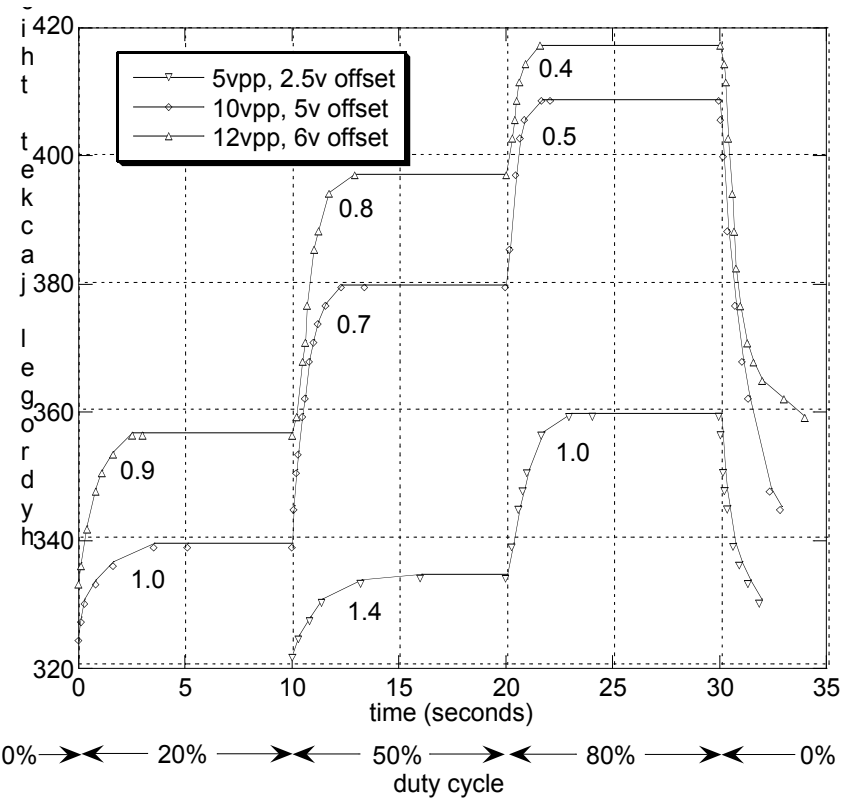

Figure 4.24. Hydrogel response curves; measured time constants are expressed in seconds next to each response. 

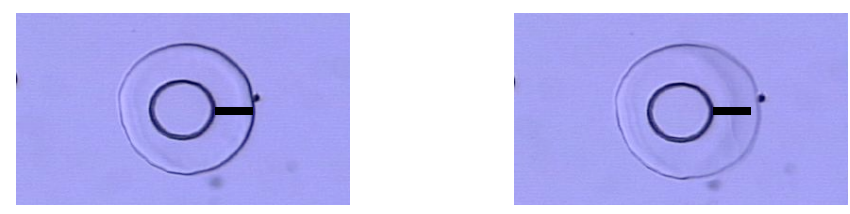

Figure 4.25. Typical hydrogel response; the response begins towards the anode and spreads across the remainder of the hydrogel. A scale bar has been added to highlight the difference in jacket thickness before and after turning the voltage on.

Using square voltage waveforms with varying pulse widths to control hydrogel volume has several advantages over previously demonstrated DC control. First, with DC control it was observed that hydrogels would continue expansion, whereas here it is seen that the hydrogels are quick to reach and hold a steady jacket thickness (Figure 4.26). Second, the amount of gas generated by electrolysis tends to be less than with DC voltages, keeping the channel from becoming blocked by bubbles.

The ability to finely tune the volume of the hydrogel with an electric field opens the door to electrically controllable valves and micropumps for flow control in microsystems; further broadening the potential uses of hydrogels in microfluidics. A device could be made to vary the fluidic resistance of a microchannel through modulation of the hydrogel volume with an electric field. If the hydrogel were positioned on a flexible membrane above a second channel, the flow could be regulated through pulse width modulation. The time response of electrically stimulated hydrogels is superior (seconds) to chemically stimulated hydrogels (minutes) for similar diffusion distances. 


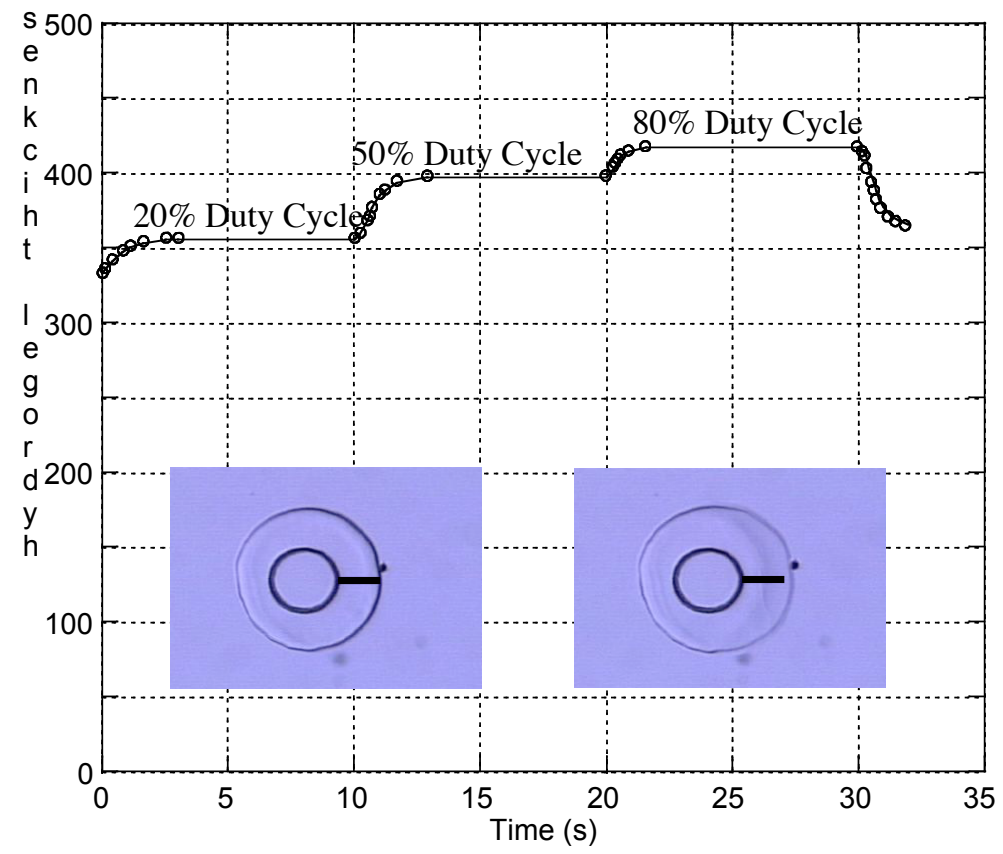

Figure 4.26. Graph of hydrogel thickness with respect to time. The duty cycle was changed as indicated on the graph. The inset pictures correspond to a $20 \%$ duty cycle and $80 \%$ duty cycle respectively. The bar on the hydrogel measures $400 \mu \mathrm{m}$. 


\section{Chapter 5 Conclusion}

The goal of this work was to create autonomous devices using photodefinable polymers and fabricated components in a self contained system. In the previous chapters, work was presented that included many materials developed during the course of this work. These materials were used to fabricate many functional components that were all made using these newly developed materials. Modeling was done on these new materials to predict their responsiveness using materials characteristics also investigated during the course of this research. We conclude here with examples of such systems created with the materials and components developed.

\section{Systems}

Several systems were developed during the course of this work, both simple (performing only one function) and complex (performing several functions).

\section{PH Self-regulation System}

A pH self-regulation system was developed that is capable of maintaining a constant $\mathrm{pH}$ given the correct flow and buffer solutions [28]. A detailed schematic of the actuation chamber, hydrogel, and star orifice is shown in Figure 5.1 for low $\mathrm{pH} 2$ buffer flow rates $(25 \mu \mathrm{L} / \mathrm{min})$ and for high flow rates $(100 \mu \mathrm{L} / \mathrm{min})$. The $\mathrm{pH} 12$ buffer passes through a four pointed star opening before entering the top channel. The star opening is shaped in this manner to throttle the amount of $\mathrm{pH} 12$ buffer solution that passes through it. The $30 \mu \mathrm{m}$ PDMS membrane closes off circular sections of the star for various $\mathrm{pH} 2$ buffer solution flows. The area under the membrane that seals the star opening is directly correlated by the size of the hydrogel that is pushing it. For a high $\mathrm{pH} 2$ flow, the hydrogel shrinks, the membrane seals off a smaller area of the star opening, and more $\mathrm{pH} 12$ buffer solution passes through. This $\mathrm{pH} 12$ buffer will meet the $\mathrm{pH} 2$ stream and because the system is operated at low Reynolds number laminar flow will cause the two streams to be stacked vertically with the $\mathrm{pH} 2$ on top and the $\mathrm{pH} 12$ on the bottom.

This vertical stacking of the streams is crucial for the system to operate correctly. The stacking causes the hydrogel post to form into a slightly conical shape as shown in Figure 5.1. Only the bottom of the hydrogel post is in the $\mathrm{pH} 12$ solution and hence, only the bottom of the post experiences any swelling and volume change. This effectively decreases the size of the hydrogel post because only the bottom experiences any change, which results in the fast time response the system needs in order to operate correctly.

As shown in Figure 5.2, the $\mathrm{pH}$ is regulated by the hydrogel sensor/actuator at $\mathrm{pH} 7$ for a $\mathrm{pH} 2$ buffer flow rates between $100-25 \mu \mathrm{L} / \mathrm{min}$. The device regulates to $\mathrm{pH} 7$ due to the transition point of the hydrogel. The hydrogel shrinks at $\mathrm{pH}$ below 5.3 and expands at $\mathrm{pH}$ above 5.3. Therefore, an equilibrium is achieved once the hydrogel is exposed to a new $\mathrm{pH} 2$ buffer flow rate. 


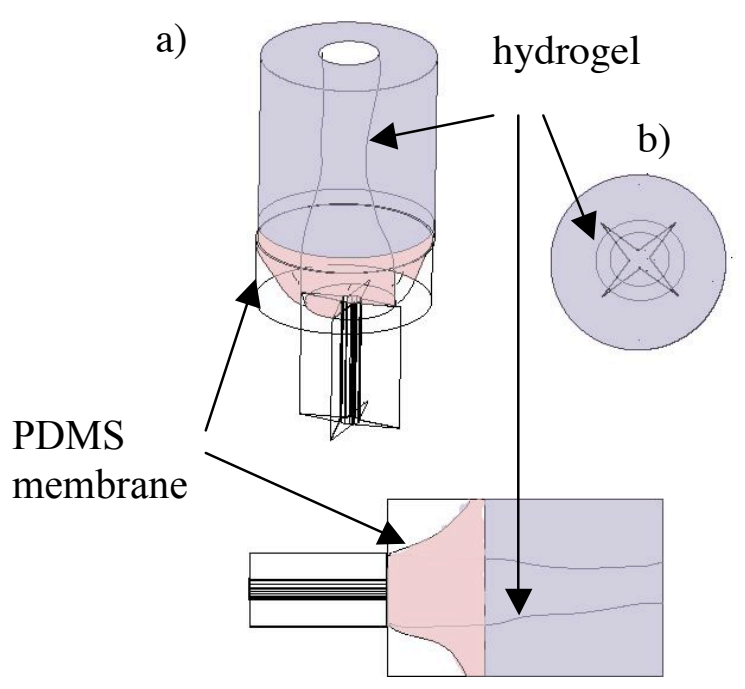

c) d)
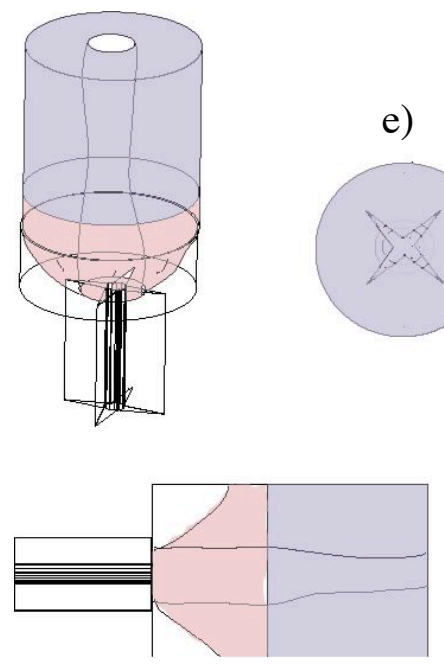

f)

Figure 5.1. Detailed drawing of the actuation chamber, hydrogel, and star orifice. a. Off axis side view, $\boldsymbol{b}$. top view, $\boldsymbol{c}$. side view of a device operating at $25 \mu \mathrm{L} / \mathrm{min} \mathrm{pH} 2$ buffer flow, $\boldsymbol{d}$. off axis side view, $\boldsymbol{e}$. top view, and $\boldsymbol{f}$. side view of a device operating at $100 \mathrm{uL} / \mathrm{min} \mathrm{pH} 2 \mathrm{buffer}$ flow rate. The pH 2 buffer is shown in blue, and the $\mathrm{pH} 12$ buffer is shown in pink. Observe the size of the hydrogel post and the resulting imprint over the star orifice at the two given flow rates.

The interface between $\mathrm{pH} 2$ buffer and $\mathrm{pH} 12$ buffer also changes with different flow rates.

The self-regulation system we have developed can be altered to regulate other variables. Other hydrogels have been developed that change in response to temperature, light, biologicals, and other hydrogels can be engineered by altering the chemistry of the hydrogel. Therefore, the hydrogel in our system can be replaced by one that responds to temperature, resulting in a constant temperature output. In the same manner, the hydrogel could be made sensitive to a biological, such as insulin, so the input stream could sample the patient's blood, and the output stream would be the desired amount of insulin in the blood. 


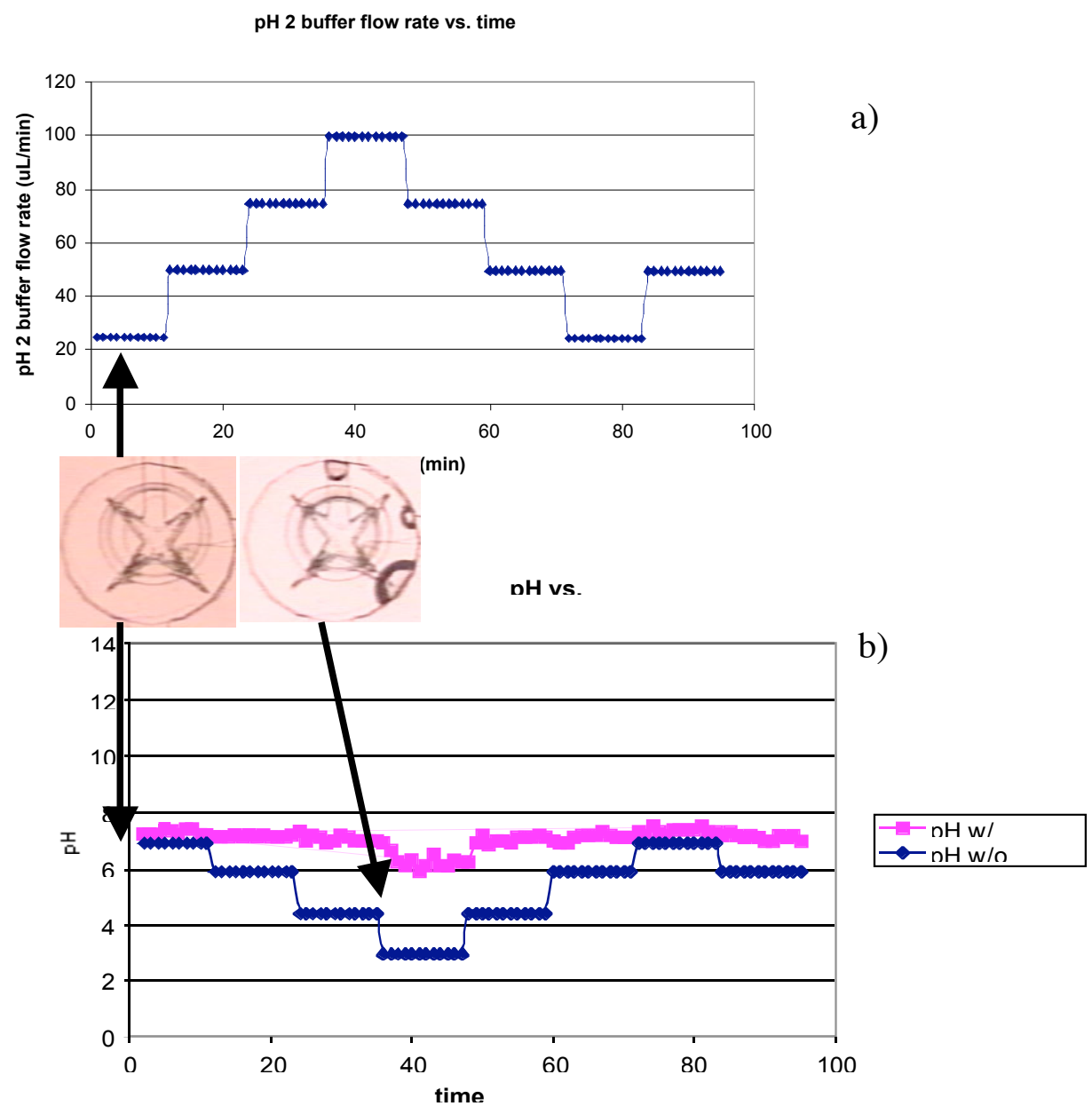

Figure 5.2. Graphs showing $\boldsymbol{a}$, Flow rate of $p H 2$ buffer $v$ s. time. $\boldsymbol{b}, \mathrm{pH}$ of the output stream $v \mathrm{~s}$ time for two separate devices, one with a hydrogel sensor/actuator and one without. The two pictures show the hydrogel sensor/actuator at two separate flow rates. The arrows point to when the picture was taken during the experiment. When the $\mathrm{pH} 2$ flow is increased, the hydrogel senses this and shrinks to allow more $\mathrm{pH} 12$ buffer through the star valve to linearize the $\mathrm{pH}$.

\section{Bioassay Development}

Botulinum toxin is produced by Clostridium botulinum, a Gram-positive, spore-forming anaerobic bacterium. Among the most potent neurotoxins known, the toxin is thought to block the release of acetylcholine from nerve endings resulting in paralysis. Although botulism is a relatively rare form of food poisoning, until recent years the rate of fatality was as high as $50 \%$. Due to improved treatments, which include antitoxin and respiratory therapy, death rates have fallen to $2 \%$, but rapid detection and typing of the toxin is imperative. The potential use of botulinum toxin as a biowarfare agent makes its rapid detection of the utmost importance. 
Additionally, while botulinum neurotoxin type A has been approved for therapeutic use in the treatment of involuntary muscle disorders, a rapid and sensitive assay for the management of optimally administered doses would prove to be a valuable tool with both clinical and research applications. The only current acceptable method for detection of botulism is the mouse toxicity and neutralization test. The test is costly in terms of the time, technical expertise required and animals needed, which, in turn, proves to be financially expensive. ELISA for BoNT is under development, but has not yet been approved by the AOAC. We have chosen the ELISA for botulinum toxin as our initial bioassay test platform. We are presently porting the assay into a "traditional" microfluidic system. This will allow us to become familiar with the assay. As we developed the $\mu \mathrm{FT}$ components, etc. they were incorporated into the micro assay system eventually leading to an all tectonics constructed system.

We chose both ELISA and non-ELISA BoNT as the main test assay for this project. The BoNT ELISA allowed us to become familiar with the current methods of detection, to pursue ELISA assay technology in a microenvironment, and to work on bio-detection in parallel with the bioresponsive hydrogel work (Figure 5.3).

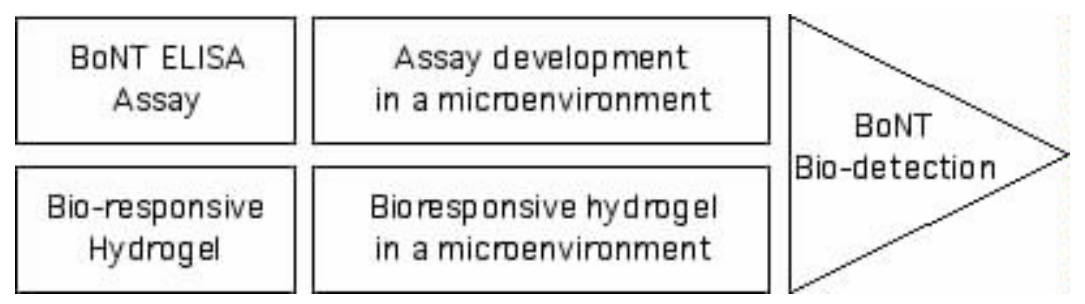

\section{Figure 5.3. Parallel BoNT bio-detection using both an ELISA assay and bio-responsive materials.}

Although the device integration of components and systems went through several iterations, the final design is presented here. Although both avenues of detection were pursued (BoNT ELISA and bioresponsive hydrogels) the method of detection using bioresponsive hydrogels proved to be too challenging to pursue. However the BoNT ELISA was successfully incorporated into the multidimensional, fully integrated, $\mu \mathrm{FT}$ universal cartridge system [29].

The platform to create a prototype microsystem for detecting botulinum neurotoxin directly from whole blood has been demonstrated. Process steps such as sample preparation by filtration, mixing and incubation with reagents was carried out on the device. Various microfluidic components such as channel network, valves and porous filter were fabricated from a prepolymer mixture consisting of monomer, cross-linker and a photo-initiator. For detection of the toxoid, biotinylated antibodies were immobilized on streptavidin-functionalized agarose gel beads. The gel beads were introduced into the device and were used as readouts. Enzymatic reaction between alkaline phosphatase (on secondary antibody) and substrate produced an insoluble colored precipitate that coated the beads thus making the readout visible to the naked 
eye. Clinically relevant amounts of the toxin can be detected from whole blood using the portable ELISA system.

One of the requirements for a portable diagnostic device is that it must be able to perform the assay directly from a biological sample such as blood, saliva or urine. To realize this capability, a sample preparation step may be necessary. The presence of botulinum neurotoxin can be tested in a blood sample; thus, the device was designed to perform the assay using whole blood. Typically, blood cells are removed from whole blood and the serum is assayed for the presence of a specific marker (antigen). In the device, a porous filter carries out this sample preparation step. The porosity of the filter depends on the pre-polymer mixture. The composition was chosen such that the size of the pores ranges from $2 \mu \mathrm{m}$ to $4 \mu \mathrm{m}$; thus making the filter efficient for removing blood cells that are about $7 \mu \mathrm{m}$ in diameter. The blood cells are compliant and can squeeze through the pores of the filter. To prevent the cells from flowing through the filter, the blood is mixed with a hypotonic solution that causes the cells to swell and become more rigid. The mixing step is performed in the chaotic mixer that is placed prior to the porous filter.

The reagents were delivered to the readout by pressing appropriate TAPs. When the TAP is released, the reagent solution tends to flow back into the TAP reservoir due to back-pressure. By incorporating check valves at appropriate locations (Figure 5.4), the backflow was prevented. Another function of the valve is to prevent contamination between the reagents. To minimize false positives, it is imperative that the secondary antibody in solution does not mix with the substrate solution. Hence the TAPs containing these two solutions were placed far away from each other (TAP2, TAP6) to minimize the chance of cross contamination. Moreover, the channels connecting these TAPs to the readout have separate valves (V2 and V4 in Figure 5.4). The wash buffer was required to remove the reagents (after incubation) and any non-specific interactions. For simplicity the wash buffer TAPs $(3,4,5)$ were placed on the same side of the device and protected from the rest of the device by one check-valve (V3). These TAPs can be used to store other reagents and additional valves could be incorporated as needed for different assays.

In the sandwich ELISA used in this study, the antigen is first bound to a biotinlyated primary antibody immobilized onto streptavidin coated beads. This step was preformed outside the device. An alkaline phosphatase toxoid binding antibody is then added and the recognition is amplified by enzymatic reaction, which enhances the sensitivity. The sensing components consist of avidin-agarose beads held by a filter membrane. Advantages in keeping the sensor and the device separate include: 1) the fabrication procedures (UV exposure and solvents) do not affect the readout and the immobilized protein, 2) the device can be pre-treated (blocked) before introducing the sensor thus minimizing background, 3) the sensor can be stored in appropriate conditions (buffer, temperature) independent of the device, and 4) the device provides the framework for detecting any other antigen depending on the antibody immobilized on the sensor. Moreover, as bead-based ELISA is becoming increasingly popular, and the device can be utilized for different applications. The readout signal is from an insoluble product formed from the reaction between alkaline phosphatase and BCIP/NBT. This substrate is commonly used in immunoblotting studies. The advantage of insoluble compounds is that the color can be easily 
seen with an unaided eye (Figure 5.4E), as opposed to spectroscopic measurements typically necessary for soluble products. However, the capability to quantify the amount of antigen is limited by availability of imaging and analysis tools.

Previously, the ability of the agarose gel beads to discern various amounts of toxoid was demonstrated. Because the color of the agarose beads increases with time, the intensity of the readout was compared 12 minutes after introducing substrate solution. A concentration dependent change in signal intensity was seen. Further optimization in utilizing the agarose solution would be necessary for quantitative assays. However, for the present qualitative assay the agarose bead is appropriate. The toxoid binding to the antibody is approximately 10 times lower than the native neurotoxin binding (compared in microtiter plates). Therefore, with the present format, detection is of the order of $1.5 \times 10^{-3}$ human $\mathrm{MLD} / \mathrm{mL}$ where MLD is the minimal lethal dose; this is a measure of the minimal dose of toxic or infectious agents that is lethal $\left(1 \mu \mathrm{g} / \mathrm{mL} \sim 15 \times 10^{-3} \mathrm{MLD} / \mathrm{mL}\right)$. By incorporating a sample concentration step and further optimizing the sensor, it should be possible to detect lower amounts of botulinum toxin with a low chance of false positives ( 1 in 250 ). Thus, the system has the ability to rapidly screen clinically relevant amounts of the neurotoxin from whole blood in the field without the need of electronics. 


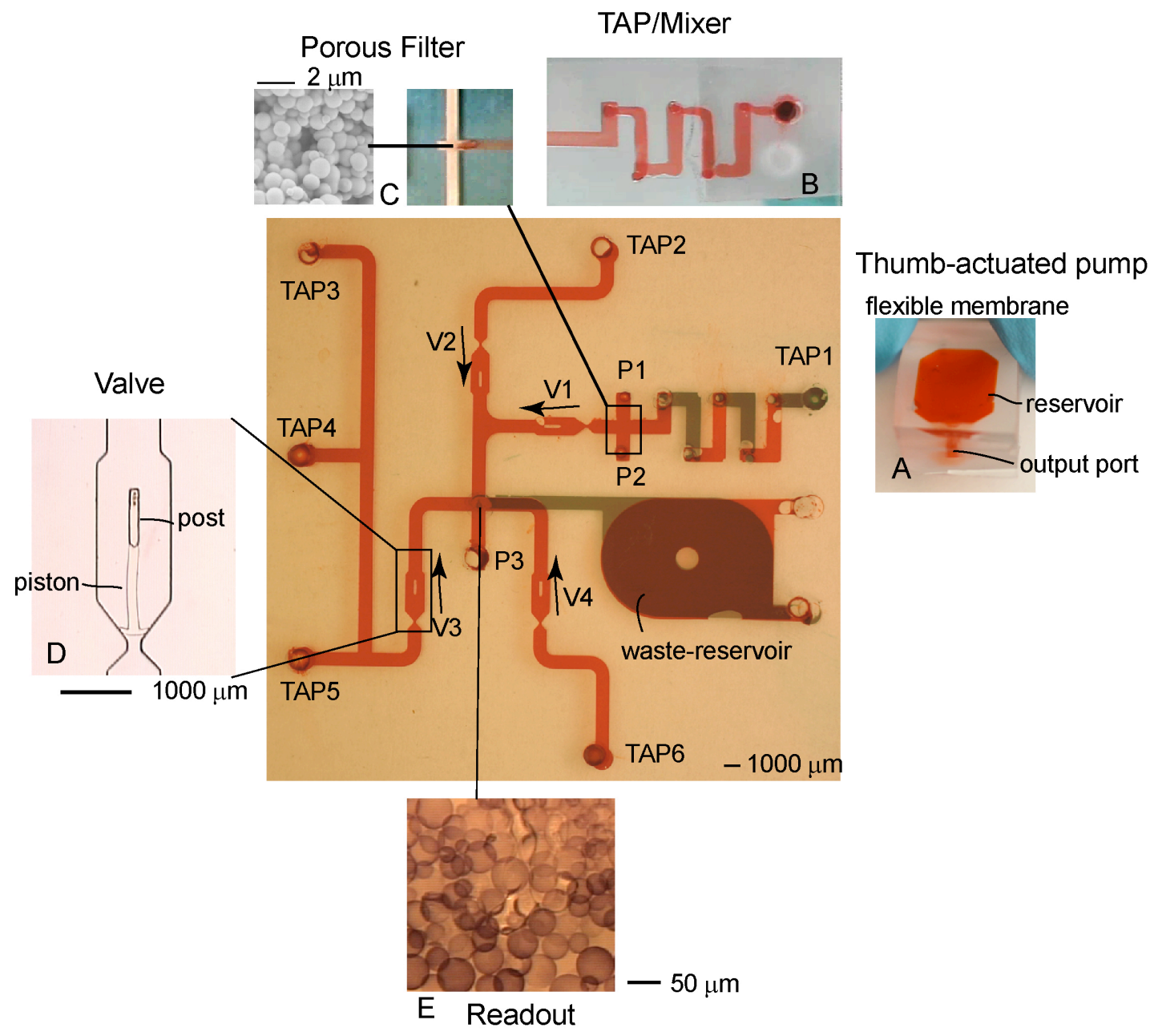

Figure 5.4. Layout of the ELISA device. All fluidic paths are filled with food color for visualization For protein storage and driving flow through the channel network, 6 TAPs were included. The TAP is micro-molded from PDMS and consists of a reservoir, an outlet port and an adhesive layer at the bottom (inset A). A chaotic mixer was fabricated between the 2 layers of the device. Fluid moving through the chaotic mixer when TAP is depressed is shown in inset B. A porous hydrogel was utilized to separate blood cells from whole blood (inset $C$ ). To prevent contamination between the reagents and backflow, 4 check-valves were fabricated in the device. Each valve consists of a rigid post and a hydrogel piston (inset D). The direction of flow allowed is indicated as arrows in the layout. The readout consists of filter membrane and agarose beads. ELISA is performed on (and in) the gel beads. The color of the beads after the assay is shown in inset $E$. 


\section{Gant Chart}

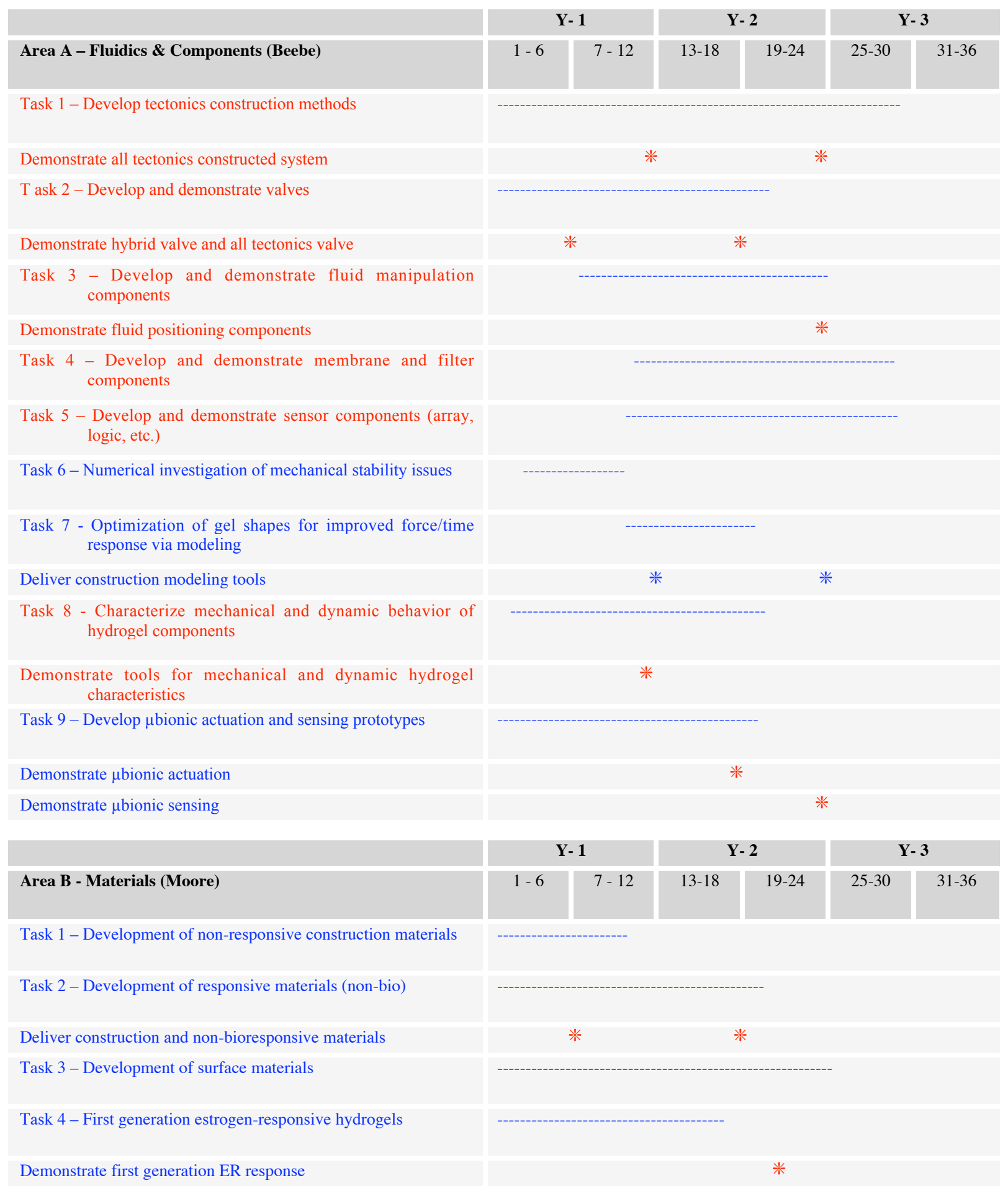




Area B - Materials (Moore) continued
Task 5 - Second generation estrogen-responsive hydrogels
Task 6 - Incorporation of E- and transcrp. Coactivator proteins
for ligand specificity
Demonstrate BC agent sensitive hydrogel (genetically eng. E-)
Task 7 - Development of BoNT responsive materials
Demonstrate BC agent hydrogels for BoNT chemistries.
Task $8 \mathrm{a}$ - Development of biomimetic cascades
Demonstrate biomimetic cascade
Task $8 \mathrm{~b}$ - Inocorporate into prototype sensor
Task 9 - Simulation of biochemical kinetics
Deliver biokinetic modeling tools

\begin{tabular}{|l|l|l|l|l|l|}
\multicolumn{2}{|c|}{ Y- 1 } & \multicolumn{2}{|c|}{ Y- 2 } & \multicolumn{2}{c|}{ Y-3 } \\
\hline $1-6$ & $7-12$ & $13-18$ & $19-24$ & $25-30$ & $31-36$ \\
\hline
\end{tabular}

Area C - Systems (Beebe)

1

\begin{tabular}{|l||c|c|c|c|c|}
\hline \multicolumn{2}{|c|}{ Y- 1 } & \multicolumn{2}{|c|}{ Y- 2 } & \multicolumn{2}{c|}{ Y-3 } \\
\hline $1-6$ & $7-12$ & $13-18$ & $19-24$ & $25-30$ & $31-36$ \\
\hline
\end{tabular}

Task 1 - Develop and demonstrate sample introduction and system control

Task 2 - Develop and demonstrate sample preparation (dilution, dialysis, etc.)

Demonstrate sample preparation module

Task 3 - Develop and demonstrate sensing systems

Demonstrate prototype sensing sub systems

Task 4 - Develop and demonstrate output configurations

Demonstrate output/display prototype

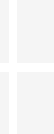

Task 5 - Integrate sub-systems and devices to fabricate a
complete BC pocket detector

Demonstrate portable system fabrication

Demonstrate prototype $\mathrm{BC}$ agent detection system

Task 6 - Construction of desk-top fabrication system

Demonstration of the desk-top fabrication of a pocket detector and the subsequent and immediate use of the detector to test a sample from a soldier

Notes: Optional tasks (not funded within initial budget) are listed in green. Tasks primarily performed at UW-Madison are listed in red, tasks primarily performed at UIUC are listed in blue. 


\section{References}

1. Beebe, D.J., et al., Microfluidic Tectonics: A comprehensive construction platform for microfluidic systems. Proc. Nat. Acad. Sci., 2000. 97(25): p. 13488-13493.

2. Beebe, D.J., et al., Functional hydrogel structures for autonomous flow control inside microfluidic channels. Nature, 2000. 404: p. 588-590.

3. Zhao, B. and J.S. Moore, Fast pH and ionic strength responsive hydrogels in microchannels. Langmuir, 2001. 17: p. 4758-4763.

4. $\quad \mathrm{Yu}, \mathrm{Q} ., \mathrm{J} . \mathrm{S}$. Moore, and D.J. Beebe. Dissolvable and asymmetric hydrogels as components for microfludic systems. in uTAS. 2002. Nara, Japan.

5. Kraft, M. and J.S. Moore, Surfactant induced lysis of lipid modified microgels. j. Am. Chem. Soc., 2001. 123: p. 1292-1922.

6. Kraft, M. and J.S. Moore, $n$-alkyl fatty acid-modified microgels: ion permeation as a function of chain length. Langmuir, 2003. 19(910-915).

7. Gimm, A., A. Ruoho, and D.J. Beebe. Biochemical singal transfer using liposomes in microfluidic channels. in uTAS. 2002. Nara, Japan.

8. Zhao, B., J.S. Moore, and D.J. Beebe, Surface directed liquid flow inside microchannels. Science, 2001. 291: p. 1023-1026.

9. Zhao, B., J.S. Moore, and D.J. Beebe, Principles of surface directed liquid flow in microfluidic channels. Anal. Chem., 2002. 74: p. 4259-4268.

10. Yung, D.Y., J.J. Magda, and I.S. Han, Macromolecules, 2000. 33: p. 3332-3336.

11. Weers, J.G., J. Fluorine Chem., 1993. 64: p. 73-93.

12. Khoury, C., G. Mensing, and D.J. Beebe, Ultra rapid prototyping of microfluidic systems using liquid phase polymerization. Lab on a Chip, 2002. 1: p. 50-55.

13. Mensing, G., T. Pearce, and D.J. Beebe, An ultra rapid method of creating $3 D$ channel networks. JALA in revision, 2004.

14. Yu, Q., et al., Responsive biomimetic hydrogel valve for microfluidics. Applied Physics Letters, 2001. 78(17): p. 2589-2591.

15. Moorthy, J. and D.J. Beebe, Tunable in situ fabricated purous filters for microsystems. Lab on a Chip, 2003. 3: p. 62-66.

16. Moorthy, J. and D.J. Beebe, A hydrogel readout for autonomous detection of ions in microchannels. Lab on a Chip, 2002. 2(2): p. 76-80.

17. Mensing, G., et al., An externally driven magnetic microstirrer. Philosophical Transactions: Mathematical, Physical, \& Engineering Sciences., 2004.

18. Eddington, D.T. and D.J. Beebe, $A$ valved responsive hydrogel micro dispensing device and storable pressure source. JMEMS in press, 2004.

19. Atencia, J. and D.J. Beebe, Magnetically-driven micro pumping using vortices. Lab on a Chip accepted, 2004.

20. Jeong, W., et al., Hydrodynamic microfabrication via "on the fly" photopolymerizationo $f$ microscale fibers and tubes. Lab on a Chip in revision, 2004.

21. De, S., et al., Equilibrium swelling and kinetics of pH-responsive hydrogels: models, experiments, and simulations. JMES, 2002. 11(5): p. 544-555. 
22. Chatterjee, A., et al., Mathematical modeling and simulation of dissolvable hydrogels. J. Aerospace Engr., 2003. 16(2): p. 55-64.

23. Bassetti, M., et al., Development and modeling of electrically-triggered hydrogels for microfluidic applications. JMEMS in revision, 2004.

24. Bashir, R., et al., Micromechanical cantilever as an ultrasensitive pH microsensor. App. Phys. Ltrs., 2002. 81(16): p. 3091-3093.

25. Johnson, B., et al., Experimental techniques for mechanical characterization of hydrogels at the microscale. Experimental Mechanics, 2003.

26. Johnson, B., W.C. Crone, and D.J. Beebe, Effects of swelling on the mechanical properties of a $\mathrm{pH}$ sensitive hydrogel. Materials Science and Engineering: C, 2003. 24: p. 575-581.

27. Bauer, J.M. and D.J. Beebe. Microscale measurements of flow bounded by air-water interfaces. in uTAS. 2002. Nara, Japan.

28. Eddington, D.T., et al., An organic self-regulating microfluidic system. Lab on a Chip, 2001. 1(2): p. 96-99.

29. Moorthy, J., et al., Microfluidic tectonics platform: a colorimetric disposable BoTox ELISA system. Electrophoresis, 2004. 25: p. 1705-1713. 


\section{Appendix A}

There were many additional publications resulting from this work. Below is a list of the publications generated from the research over the duration of this project.

1. J. Moorthy and D. J. Beebe, "Protein interaction analysis: microfluidic systems," in Encyl. Of Proteomics and Genomics, submitted 2004.

2. D. T. Eddington and D. J. Beebe, "Development of a disposable infusion system fro the delivery of protein therapeutics," IEEE Trans Biomed Eng. Submitted, 2004.

3. D. T. Eddington and D. J. Beebe, "Flow control with hydrogels", 56 199-210, Advanced Drug Delivery Reviews, 2004.

4. J. Moorthy and D. J. Beebe, "Organic and Bio-mimetic Approaches to Design Microfluidic Systems", Anal. Chem. 75, 2003.

5. S. Lee, D. T. Eddington, Y. Kim, W. Kim, and D. J. Beebe"Control Mechanism of an Organic Self-Regulating Microfluidic System", submitted to JMEMS.

6. J. Bauer, T. A. Saif, and D. J. Beebe, "Surface tension driven formation of microstructures," JMEMS, 13, 553-558, 2004

7. J. Moorthy and D. J. Beebe, "Organic and bio-mimetic approaches in designing microfluidic systems," Anal. Chem, 75, 13, 292-301, 2003.

8. D. Eddington, W. Crone, and D. J. Beebe, "Development of process protocols to fine tune Polydimethylsiloxane material properties", $\mu$ TAS, Oct. 5-9, Squaw Valley, CA 2003.

9. J. Atencia and D. J. Beebe, "An oscillating ferromagnetic micropump utilizing a centrifugal force ", $\mu$ TAS, Oct. 5-9, Squaw Valley, CA 2003.

10. D. Kim and D. J. Beebe, "In-Situ Fabricated Micro Check-Valve Utilizing the Spring Force of a Hydrogel ", $\mu$ TAS, Oct. 5-9, Squaw Valley, CA 2003.

11. G. Mensing, D. Kim, J. Moorthy, J. Bassett, D. Eddington, and D. J. Beebe, “A reconfigurable integrated device for bioassay development", $\mu$ TAS, Oct. 5-9, Squaw Valley, CA 2003.

12. C. Khoury, T. Adalsteinsson, B. Johnson, W.C. Crone, D.J. Beebe, "Tunable Microfabricated Hydrogels A Study in Protein Interaction and Diffusion," Biomedical Microdevices, 5, 1, 35-45 (2003).

13. J. Bauer and D. J. Beebe, "Liquid Phase Construction of Microstructures," submitted to JMEMS.

14. R. Vijayendran, K. Motsegood, D. J. Beebe and D. Leckband, "Evaluation of a ThreeDimensional Micromixer in a Surface-Based Biosensor," Langmuir, 1824-1828, 2003.

15. B. Zhao, J. S. Moore, and D. J. Beebe, Pressure-Sensitive Microfluidic Gates Fabricated by Patterning Surface Free Energies Inside Microchannels", Langmuir, 1873-1879, 2003.

16. S. Dineen, M. Bradshaw, and E. Johnson, "Neurotoxin Gene Clusters in Clostridium botulinum Type A stains: Sequence Comparison and Evolutionary Implications", accepted to Curr. Microbio. 
17. B. Zhao, N. O. L. Viernes, J. S. Moore, D. J. Beebe, "Control and Applications of Immiscible Liquids in Microchannels," J. Am. Chem. Soc 124 2003, 5284-5285.

18. K. N. Plunkett, M. L. Kraft, Q. Yu, J. S. Moore, "Swelling kinetics of disulfide crosslinked microgels" Macromolecules, 36, 3960-3966, 2003.

19. H. J. van der Linden, D. J. Beebe, and P. Bergveld, "A temperature controlled microvalve for biomedical applications using a temperature sensitive hydrogel", $\mu T A S$ Nov. 3-7, Nara, Japan, 2002, pp. 142-144.

20. G. Mensing and D. J. Beebe, "Liquid phase 3D channel networks", $\mu T A S$ Nov. 3-7, Nara, Japan, 2002, pp. 410-412.

21. M. Kraft, D. J. Beebe, and J. S. Moore, "Surface modification of microgels with N-alkyl fatty acid layers: ion gradient properties", $\mu T A S$ Nov. 3-7, Nara, Japan, 2002, pp. 428430.

22. M. J. Bassetti and D. J. Beebe, "Demonstration of hydrogel volume control using pulse width modulation", $\mu T A S$ Nov. 3-7, Nara, Japan, 2002, pp. 718-720.

23. D. T. Eddington, D. J. Beebe, "A hydrogel actuated microdispensing device," IEEE EMBS \& BMES Conf., Oct. 2002.

24. J. Bassett, G. Walker, D. J. Beebe, "Mycological culture in microchannels," IEEE EMBS \& BMES Conf., Houston, TX, Oct. 2002.

25. D. J. Quirk, D. J. Beebe, "Photolithographic fabrication of three-dimensional microfluidic devices using multiple masks, IEEE EMBS \& BMES Conf., Houston, TX, Oct. 2002.

26. G. Walker and D. J. Beebe, "An evaporation-based microfluidic sample concentration method," Lab on a Chip, vol. 2, 2002, pp. 57-61.

27. J. Moorthy and D. J. Beebe, "In-situ fabricated porous filter-characterization and biological applications," IEEE EMBS MMB Conf., May 2-4, Madison, WI, 2002, pp. 514-517.

28. G. Mensing, T. Pearce, and D. J. Beebe, "An in-plane active magnetic mixer for microfluidic applications,” IEEE EMBS MMB Conf. May 2-4, Madison, WI, 2002, pp. 531-534.

29. C. Khoury, T. Adalsteinson, and D. J. Beebe, "Characterization of protein interaction with tunable microfabricated hydrogels," IEEE EMBS MMB Conf. May 2-4, Madison, WI, 2002, pp. 400-405.

30. M. J. Bassetti, J. S. Moore, and D. J. Beebe, "Development of electrically triggered hydrogels for microfluidic applications," IEEE EMBS MMB Conf. May 2-4, Madison, WI, 2002, pp. 410-413.

31. G. Walker and D. J. Beebe, "Evaporation driven microfluidic sample concentration," IEEE EMBS MMB Conf. May 2-4, Madison, WI, 2002, pp. 523-526.

32. R. H. Liu, Q. Yu, and D. J., Beebe, "Fabrication and characterization of hydrogel-based microvalves," JMEMS, 11, 2002, pp. 45-53.

33. M. C. Goodnough, G. Oyler, P. S. Fishman, E. A. Johnson, E. A. Neale, J. E. Keller, W. H. Tepp, M. Clark, S. Hartz and M. Adler, "Development of a delivery vehicle for intracellular transport of botulinum neurotoxin antagonists," FEBS Letters, 513, 2-3, 2002, pp. 163-168. 
34. D. J. Beebe, G. Mensing, and G. Walker, "Physics and application of $\mu$ fluidics in biology." In Yarmush (ed.), Annual Review of Biomedical Engineering, 2002, 4: pp. 261-286.

35. D. J. Beebe, G. Mensing, J. Moorthy, C. Khoury, and T. Pearce, "Alternative approaches to microfluidic systems design, construction, and operation," $\mu T A S$ October 21-25, Monterey, CA, 2001, pp. 453-455.

36. D. Eddington, Liu, R. H.; Moore, J. S.; and D. J Beebe, "Regulation of pH in a microfluidic stream," $\mu T A S$ October 21-25, Monterey, CA, 2001, pp. 486-488.

37. J. Moorthy, and D. J. Beebe, "Autonomous colorimetric readout for microfluidic devices," $\mu T A S$ October 21-25, Monterey, CA, 2001, pp. 173-174.

38. G. Mensing, and D. J. Beebe, "Construction and application of responsive microstructures: Tubes and sensors," $\mu T A S$ October 21-25, Monterey, CA, 2001, pp. 169-170.

39. H. M. Solomon, E. A. Johnson, D. T. Bernard, S. S. Arnon, and J. L. Ferreira, "Clostridium botulinum and its toxins," In: F. P. Downes and K. Ito, (Eds.), Compendium for the Microbiological Examination of Foods, 4th edition. American Public Health Association, Washington, DC, 2001, pp. 317-324.

40. E. A. Johnson, "Clostridia botulinum and its neurotoxins: a metabolic and cellular perspective," Toxicon, 39, 2001, pp. 1703-1722.

41. J. Moorthy, C. Khoury, J. S. Moore, and D. J. Beebe, "Active Control of Electroosmotic Flow in Microchannels Using Light," Sens. Actuators, B, vol. 75, 3, 2001, pp. 223-229.

42. E. A. Johnson, "Clostridia", In: J. Lederberg, (Editor in Chief), Encyclopedia of Microbiology, 2nd edition, Academic Press, NY, 2000.

43. M. G. Olson, J. M. Bauer, D. J Beebe, "Particle imaging technique for measuring the deformation rate of hydrogel microstructures" App. Phys. Lett. 76, 22, 2000, pp. 33103312. 